REVIEWS OF MODERN PHYSICS, VOLUME 89, JANUARY-MARCH 2017

\title{
Equations of state for supernovae and compact stars
}

\author{
M. Oertel \\ Laboratoire Univers et Théories, CNRS, Observatoire de Paris, \\ PSL Research University, Université Paris Diderot, Sorbonne Paris Cité, \\ 5 place Jules Janssen, 92195 Meudon, France \\ M. Hempel \\ Departement Physik, Universität Basel, Klingelbergstrasse 82, 4056 Basel, Switzerland
}

T. Klähn

Instytut Fizyki Teoretycznej, Uniwersytet Wrocławski, pl. M. Borna 9, PL-50-204 Wrocław, Poland

\author{
S. Typel ${ }^{\S}$ \\ Institut für Kernphysik, Technische Universität Darmstadt, \\ Schlossgartenstraße 9, 64289 Darmstadt, Germany \\ and GSI Helmholtzzentrum für Schwerionenforschung, Planckstraße 1, \\ 64291 Darmstadt, Germany
}

(published 15 March 2017)

A review is given of various theoretical approaches for the equation of state (EoS) of dense matter, relevant for the description of core-collapse supernovae, compact stars, and compact star mergers. The emphasis is put on models that are applicable to all of these scenarios. Such EoS models have to cover large ranges in baryon number density, temperature, and isospin asymmetry. The characteristics of matter change dramatically within these ranges, from a mixture of nucleons, nuclei, and electrons to uniform, strongly interacting matter containing nucleons, and possibly other particles such as hyperons or quarks. As the development of an EoS requires joint efforts from many directions, different theoretical approaches are considered and relevant experimental and observational constraints which provide insights for future research are discussed. Finally, results from applications of the discussed EoS models are summarized.

DOI: 10.1103/RevModPhys.89.015007

\section{CONTENTS}

I. Introduction

II. General Remarks on the EoS

A. Basic thermodynamic considerations

B. Specific requirements for astrophysical EoSs

1. Equilibrium conditions

2. Charge neutrality and inhomogeneity effects

3. Range of thermodynamic variables

4. Particle degrees of freedom

III. Formal Approaches to the Description of Dense Matter

A. Basic few-body interactions

1. Experimental data

2. Phenomenological forces

a. Meson-exchange models

b. Potential models

3. Interactions from chiral effective field theory and lattice QCD

*micaela.oertel@obspm.fr

${ }^{\dagger}$ matthias.hempel@unibas.ch

thomas.klaehn@ift.uni.wroc.pl

§s.typel@gsi.de
4. Renormalization group methods and evolved potentials

B. Many-body methods for homogeneous matter 9

1. Ab initio methods 10

a. Self-consistent Green's function $\quad 10$

b. Brueckner-Hartree-Fock 11

c. Methods derived from the variational principle $\quad 12$

d. Quantum Monte Carlo methods 13

e. Chiral effective field theory 13

f. Lattice methods 13

g. Perturbative QCD 14

h. Dyson-Schwinger approach 14

2. Phenomenological approaches 14

a. Hadronic matter 15

b. Quark matter 18

C. Clustered and nonuniform matter 19

1. Nuclear statistical equilibrium 19

a. Nuclear binding energies $\quad 20$

b. Excited states 20

c. Coulomb interaction 20

d. Medium modifications of heavy nuclei 20

e. Cluster dissolution 20

2. Single nucleus approximation 21 
M. Oertel et al.: Equations of state for supernovae and compact ...

3. Virial expansion

4. Quantum statistical approach

5. Generalized relativistic density functional

6. Nucleons-in-cell calculations

D. Phase transitions

1. Thermodynamic description of phase transitions

2. Coulomb effects

IV. Constraints on the EoS

A. Terrestrial experiments

1. Systematics from nuclear masses and excitations

2. Nuclear resonances

a. Giant monopole resonance

b. Giant dipole resonance

c. Electric dipole polarizability

3. Neutron skin thicknesses

4. Heavy-ion collisions

B. Neutron matter calculations

C. Astrophysical observations

1. Neutron star masses and radii

2. Neutron star cooling and rotation

D. Summary of constraints on the symmetry energy

V. Modeling the EoS

A. Neutron star EoS

1. Neutron star crust EoSs and unified neutron star EoSs

2. Composition of the neutron star core

B. EoS of uniform matter at finite temperature

C. EoS of clustered matter at finite temperatures

D. General purpose equations of state

1. Nucleons and nuclei as degrees of freedom

a. $\mathrm{H} \& \mathrm{~W}$

b. LS

c. STOS

d. FYSS

e. HS

f. SFHo and SFHx

g. SHT(NL3), SHO(FSU), and SHO(FSU2.1)

2. Including additional degrees of freedom

3. Compatibility with experimental and observational constraints

VI. Applications in Astrophysics

A. Binaries and binary mergers

B. Core-collapse supernovæ

1. Dynamics

2. PNSs, neutrino-driven winds, and nucleosynthesis 5

3. Black hole formation 52

VII. Summary and Conclusions

Acknowledgments

Appendix: Resources

1. EoS databases

2. Open source simulation software

References

\section{INTRODUCTION}

Matter under extreme conditions can be found at various places in the Universe. Extremely high densities exist in neutron stars (NSs) (Glendenning, 1997; Weber, 2005; Haensel, Potekhin, and Yakovlev, 2007; Potekhin, 2010; Lattimer, 2012). Densities above nuclear saturation density and high temperatures are reached when the core of a massive star collapses. The resulting core-collapse supernova (CCSN) explosion (Mezzacappa, 2005; Kotake, Sato, and Takahashi, 2006; Janka et al., 2007; Ott, 2009; Janka, 2012a; Burrows, 2013) leads to the formation of a protoneutron star (PNS) (Prakash et al., 1997; Pons et al., 1999) and finally to a NS or a black hole $(\mathrm{BH})$. Similar densities and temperatures, but higher isospin asymmetries (viz., a higher excess of neutrons over protons), are involved in the merging of NSs in close binary systems, NS-NS, and NS-BH (Shibata and Taniguchi, 2011; Faber and Rasio, 2012; Rosswog, 2015). The dynamical evolution of such violent events and the structure of the emerging compact stars are determined among others by the equation of state $(\mathrm{EoS})$ of matter. In addition, the EoS impacts the conditions for nucleosynthesis and the emerging neutrino spectra. Hence, the EoS is an essential ingredient in many astrophysical simulations. ${ }^{1}$ Many efforts are made to gain a comprehensive understanding of properties of the involved matter, which in several aspects are dramatically different from those in terrestrial experiments.

During the last decades numerous theoretical investigations, laboratory experiments, as well as astronomical observations have been conducted in order to constrain the thermodynamic properties and chemical composition of stellar matter for conditions relevant to the description of compact stars, CCSNe, and NS mergers; see, e.g., Klähn et al. (2006), Lattimer and Prakash (2007), and Lattimer and Lim (2013). There is an intrinsic connection between the macroscopic structure and evolution of such astrophysical objects and the underlying fundamental interactions between the constituent particles at the microscopic level. This makes the study of the aforementioned systems very rewarding as they challenge our understanding of nature on both scales. The aim of this paper is to review existing approaches for the description of dense matter that can yield EoSs relevant for compact star astrophysics from both purely theoretical and phenomenological perspectives. There is a large number of different approaches. In many cases the properties of matter can be provided only for particular thermodynamic conditions, not always sufficient to describe simultaneously all the astrophysical systems we address. Hence, the main emphasis of this work is placed on the discussion of approaches to the EoS that are readily available for use in astrophysical simulations. Such EoSs that cover the full thermodynamic parameter range of temperature, density, and isospin asymmetry relevant for CCSNe, NSs, and compact binary mergers we call "general purpose EoSs."

Within this review we will not consider the EoS for white dwarfs since, although being compact stars in astronomical terminology, the underlying microphysics is quite different. Because of the complexity of the topic we will also not discuss pairing and related effects of superfluidity and superconductivity; see, e.g., Lombardo and Schulze (2001), Alford et al. (2008), Chamel and Haensel (2008), and Page et al. (2014) for corresponding reviews.

\footnotetext{
${ }^{1}$ Within this review we employ the general term "astrophysical simulations" or "astrophysical applications" synonymously with "astrophysical simulations of CCSNe, (proto)NSs, and compact binary mergers involving NSs."
} 
In Sec. II some basic thermodynamic considerations, definitions, and requirements for an EoS in astrophysical applications are discussed. In order to get an idea about the challenge of constructing such an EoS, it is useful to state the relevant degrees of freedom and the ranges of the thermodynamic variables that have to be covered.

It is not an easy task to obtain a reliable description of dense matter that covers the full range of thermodynamic variables. In Sec. III formal approaches to the description of dense matter are discussed. The main uncertainties arise from two sources as follows:

(1) the, at least partly, poor knowledge of the interactions, and

(2) the treatment of the many-body problem for strongly interacting particles.

Basic considerations about interactions are presented in Sec. III.A. In Secs. III.B and III.C we outline currently available techniques that address the many-body problem at finite densities and temperatures for homogeneous and inhomogeneous matter. We briefly emphasize their respective advantages and current limitations. The description of inhomogeneous matter is particularly important for CCSNe. It is conceptually and computationally very involved and up to now it has not been possible to apply sophisticated $a b$ initio many-body methods on a grand scale. Finally, Sec. III.D discusses specific features that appear in the treatment of phase transitions.

The inherent uncertainties of any EoS model require a careful analysis of and comparison with available experimental and observational data. Therefore, we give an overview of constraints of the EoS from terrestrial experiments, theoretical considerations, and astrophysical observations in Sec. IV.

Section V presents an overview of EoS models for astrophysical applications. Since EoSs for cold compact stars have been discussed extensively in the literature, only a summary of available models and their main features is given. The main emphasis is put on currently existing general purpose EoS.

Section VI summarizes the impact of the EoS on the astrophysics of compact stellar objects, e.g., on (proto)NSs, binary mergers, CCSNe, and the formation of BHs. The main aim of this section is to show how different parts of the EoS and the associated uncertainties are related to potential astrophysical observations. These considerations might be useful to identify open questions which inspire further work for improving EoS models.

The Appendix lists freely available resources, databases, and software, which are related to the EoS and its application in the astrophysics of compact stellar objects.

Throughout this paper we use units where $k_{B}=\hbar=c=1$.

\section{GENERAL REMARKS ON THE EOS}

\section{A. Basic thermodynamic considerations}

In its most general form the expression "equation of state" is used for any relation between thermodynamic state variables. Depending on the context, often we use it more specifically for the set of thermodynamic equations that fully specifies the state of matter under a given set of physical conditions.

EoSs are typically employed in astrophysical models that use a hydrodynamic description of the macroscopic system. In this case, it is assumed that matter can be considered as a fluid, and explicit effects from the gravitational field do not have to be included in the thermodynamic description. The construction of an EoS supposes that the local system under consideration is in thermodynamic equilibrium. This usually means that intensive thermodynamic variables such as temperature, pressure, or chemical potentials are well defined and that the conditions of thermal equilibrium (equivalent to a constant temperature throughout the chosen domain), mechanical equilibrium (constant pressure), and chemical equilibrium (constant chemical potentials) hold. Therefore, uniformity of all independent intensive variables has to be demanded. In order to obtain a thermodynamically consistent approach, it is most convenient to start from a thermodynamic potential, chosen according to the set of natural variables used, and to derive all relevant quantities by standard thermodynamic relations; see, e.g., Landau and Lifshitz (1980) or the CompOSE manual (Klähn, Oertel, and Typel, 2013; Typel, Oertel, and Klähn, 2015). An example is the Helmholtz free energy $F\left(T,\left\{N_{i}\right\}, V\right)$ [used, e.g., by Lattimer and Swesty (1991)], depending on the natural variables temperature $T$, the set of particle numbers $N_{i}\left(i=1, \ldots, N_{\text {part }}\right)$, and the volume $V$. It attains a minimum in the ground state of the system for given values of the thermodynamic variables. In the thermodynamic limit, the actual value of $V$ is irrelevant, and all extensive variables follow the same scaling. Therefore one can work with ratios of extensive variable such as the particle number densities $n_{i}=N_{i} / V$ that behave as intensive variables. In general, the different particle species $i$ are not inert but can convert to other species by reactions. If they are in equilibrium the state of the system is characterized by a number $N_{\text {cons }} \leq N_{\text {part }}$ of independent conserved charges. Thus, in general, the individual particle densities $n_{i}$ are not independent, but connected by conditions of chemical equilibrium that can be expressed with the help of the particle chemical potentials $\mu_{i}=\partial F / \partial N_{i}$. Sometimes a theoretical description that starts from the grand canonical potential $\Omega\left(T,\left\{\mu_{i}\right\}, V\right)=F-\sum_{i} \mu_{i} N_{i}$ is more convenient.

\section{B. Specific requirements for astrophysical EoSs}

\section{Equilibrium conditions}

An EoS can be applied only if the system is in thermodynamic equilibrium. In astrophysical simulations, this concerns, in particular, the chemical equilibrium since thermal and mechanical equilibrium are in general quickly achieved with $T$ and $p$ as the associated intensive variables. Then, the use of an EoS in chemical equilibrium is justified only if the time scales of the corresponding reactions are much shorter than the time scales of the system's hydrodynamic evolution.

Chemical equilibrium among different nuclear species is not achieved, if an ensemble of nuclei, nucleons, and electrons is considered at densities and temperatures as reached in main sequence stars, in the outer regions of a CCSN or in explosive 
nucleosynthesis. Hence the time evolution of the composition has to be followed with a reaction network depending on the reaction cross sections of the participating particle species. Typically it is assumed that a temperature on the order of $0.5 \mathrm{MeV}$ and above is sufficient to reach the so-called nuclear statistical equilibrium (NSE) (Iliadis, 2007).

A typical set of conserved charges of the system are the total baryon number $N_{B}$, the total (electric) charge number $N_{Q}$, the total electronic lepton number $N_{L^{(e)}}$, and the total strangeness number $N_{S}$. The quantities $N_{i}$ are thereby defined as net particle numbers. Correspondingly, for every particle the chemical potential is given by

$$
\mu_{i}=B_{i} \mu_{B}+Q_{i} \mu_{Q}+L_{i}^{(e)} \mu_{L_{e}}+S_{i} \mu_{S}
$$

with the baryon $\left(B_{i}\right)$, charge $\left(Q_{i}\right)$, electronic lepton $\left(L_{i}^{(e)}\right)$, and strangeness number $\left(S_{i}\right)$ of the individual particle. Hence, the specification of the baryon chemical potential $\mu_{B}$, the charge chemical potential $\mu_{Q}$, the electronic lepton chemical potential $\mu_{L_{e}}$, and the strangeness chemical potential $\mu_{S}$ is sufficient to obtain the chemical potential of every constituent. In particular, in NSE, the chemical potential of each nucleus $a$ with neutron number $N_{a}$ and proton number $Z_{a}$ is given by

$$
\mu_{a}=\left(N_{a}+Z_{a}\right) \mu_{B}+Z_{a} \mu_{Q} \equiv N_{a} \mu_{n}+Z_{a} \mu_{p},
$$

where $\mu_{n}\left(\mu_{p}\right)$ is the chemical potential of neutrons (protons). Conditions on (electric) charge neutrality and weak equilibrium can further reduce the number of independent particle numbers or chemical potentials.

Weak interactions, for instance the electron-capture reaction $p+e^{-} \rightarrow n+\nu_{e}$, cannot be considered in equilibrium in general, since the relevant time scales can exceed the dynamical time scale of the astrophysical object of interest. In particular, in CCSNe, except at the highest densities roughly above $n_{B}=N_{B} / V=10^{-3} \mathrm{fm}^{-3}$, no weak equilibrium is obtained. In addition, neutrinos are not necessarily in equilibrium, neither thermal nor chemical. Usually they are not considered within the EoS, but treated via a transport approach. The neutrino transport equations, together with the employed weak interaction rates, are then coupled via energy, momentum, and lepton number conservation to the hydrodynamic evolution of the system and to the EoS. They determine the electron number densities, which remain a degree of freedom of the EoS. Concerning strangeness changing weak interactions, in the temperature and density range where strange particles have non-negligible abundances, the time scales estimated for the relevant processes are of the order of $10^{-6} \mathrm{~s}$ or below; see, e.g., Brown et al. (1992). Therefore, in general strangeness changing weak equilibrium is assumed, i.e., $\mu_{S}=0$. Hence, strangeness is not a conserved charge and does not represent an independent thermodynamic variable.

The situation is different for the highest densities reached in CCSNe, i.e., in hot (proto)NSs. At the prevailing high temperatures and densities, neutrinos are trapped and equilibrium with respect to weak reactions is achieved. They can be treated as part of the EoS, parametrized by the neutrino fraction $Y_{\nu_{e}}=n_{\nu_{e}} / n_{B}$ or the lepton fraction $Y_{L_{e}}=Y_{\nu_{e}}+Y_{e}$ with the electron fraction $Y_{e}=n_{e} / n_{B}$. At a later cooling stage neutrinos become untrapped, i.e., their mean free path becomes longer than the system size and $\beta$ equilibrium without neutrinos is established. This condition can be expressed by setting the electronic lepton chemical potential $\mu_{L_{e}}$ to zero in Eq. (1) as for cold NSs. Together with charge neutrality it implies that $n_{e}$ or $Y_{e}$ are fixed by $n_{B}$ and are no longer free variables of the EoS.

Assuming lepton flavor conversion via neutrino oscillations to be negligible, the heavy flavor lepton numbers are conserved independently of the electronic lepton number. For the moment no simulation has been performed that includes heavy charged leptons explicitly. The influence of heavy flavor leptons is expected to be small due to their high rest masses. Nevertheless, in EoSs of cold NSs muons are usually included.

\section{Charge neutrality and inhomogeneity effects}

In all astrophysical scenarios considered in this review, the system can be regarded as infinitely large on the length scales of the microscopic model. The thermodynamic limit is reached and electric charge neutrality is required to avoid instabilities due to the occurrence of strong electric fields.

In its simplest form, charge neutrality can be formulated as a local condition $n_{Q}=\sum_{i} Q_{i} n_{i}=0$. Thus $n_{Q}$ is not an independent thermodynamic degree of freedom and it is convenient to introduce the hadronic charge density $n_{q}=\sum_{i}{ }^{\prime} Q_{i} n_{i}$, where the primed sum runs over all hadrons (and/or quarks, if present). If electrons are the only leptonic component, this implies $n_{q}=n_{e}$.

In the case of inhomogeneous matter, the charge distribution can be imbalanced locally. The resulting competition between nuclear surface and Coulomb energies causes the formation of clusters or more complicated structures such as "pasta phases" (Ravenhall, Pethick, and Wilson, 1983; Hashimoto, Seki, and Yamada, 1984; Williams and Koonin, 1985). Charge neutrality is maintained only globally. In a simple approximation, this occurrence of finite-size structures with low and high baryon number densities can be treated as a coexistence of phases, however, surface and Coulomb effects are neglected in this case (see Sec. III.D for details).

\section{Range of thermodynamic variables}

The most general case we are interested in is an EoS depending on the temperature $T$, total baryon number density $n_{B}$, and the total hadronic charge density $n_{q}$ or a set of equivalent thermodynamic variables. Instead of $n_{q}$, the corresponding fraction $Y_{q}=n_{q} / n_{B}$ may be used. The baryon number density $n_{B}$ is sometimes replaced by the mass density $\varrho=m_{B} n_{B}$ with the mass unit $m_{B}$, which is often taken to be either the atomic mass unit $m_{u}$ or the neutron mass $m_{n}$. As an order of magnitude estimate, a baryon number density of $0.1 \mathrm{fm}^{-3}$ corresponds to a mass density of $\varrho \approx 1.66 \times 10^{14} \mathrm{~g} / \mathrm{cm}^{3}$.

The observations of compact stars with masses of $2 M_{\odot}$ (Demorest et al., 2010; Antoniadis et al., 2013; Fonseca et al., 2016) imply that the maximum baryon number density in NSs can approach approximately 10 times the nuclear saturation density $n_{\text {sat }} \approx 0.16 \mathrm{fm}^{-3}$ (Lattimer and Prakash, 2011). The 
densities in $\mathrm{CCSNe}$ and PNSs are generally lower. An exception is the case of so-called "failed" CCSNe leading to BH formation (see Sec. VI.B.3). During the final collapse to a $\mathrm{BH}$, densities well above $10 n_{\text {sat }}$ can be reached before the formation of an event horizon (Sumiyoshi, Yamada, and Suzuki, 2007; O'Connor and Ott, 2011; Hempel et al., 2012; Peres, Oertel, and Novak, 2013). However, these extremely high densities occur only for less than a millisecond. The free-fall-like collapse to a BH is largely dominated by gravity and the EoS is not expected to influence its dynamics.

For the gross structure of NSs, the state of matter below $10^{-11} \mathrm{fm}^{-3}$ is practically irrelevant, as it makes up only the few outermost centimeters of the star. In CCSNe, and to some extent also in NS mergers, the situation is different. Here lowdensity matter plays an important role. In both cases one is interested in the ejecta. Their densities decrease continuously during their expansion. Typically, a simple ideal gas EoS is used for the description of matter under such conditions; however, full thermodynamic equilibrium (see Sec. II.B.1) cannot always be assumed. Instead a network of timedependent nuclear reactions has to be considered. For $\mathrm{CCSNe}$, the ongoing burning processes, which contribute to the final explosion energies (Yamamoto et al., 2013; Perego et al., 2015), occur in low-density matter. To follow the evolution of a CCSNe for several seconds, it is unavoidable to include a description of low-density matter out of NSE. From a practical point of view, the connection of such a region to a tabulated EoS in the higher-density and temperature regime can be quite intricate.

The temperature of a typical NS older than a few minutes is small (below $1 \mathrm{MeV}$ ) on nuclear energy scales (Pons et al., 1999; Suwa, 2014) and can be considered as zero in most applications. However, these objects are born in CCSNe which can be extremely hot events. The same holds for NS-NS and NS-BH mergers. Typical temperatures in CCSNe and PNS are in the range from a fraction to a few tens of $\mathrm{MeV}$. This can be inferred from Fig. 1 which shows the temperatures and densities reached during a CCSN simulation for the $15 M_{\odot}$ progenitor of Woosley and Weaver (1995) within the first second after bounce. It is a typical example for the core collapse of an intermediate-mass progenitor which is expected to lead to an explosion. The temperatures obtained correspond to entropies per baryon in the range from 1 to 5 at the stage of collapse and up to 20 in the shock-heated matter. For other progenitors that are also expected to lead to explosions, the range of temperatures is similar. Scenarios with $\mathrm{BH}$ formation set the upper limits for density and temperature which a general purpose EoS has to cover. The temperature in such an event can rise above $100 \mathrm{MeV}$; see, e.g., O'Connor and Ott (2011). Therefore the temperature domain to be covered by a general purpose EoS is $0 \mathrm{MeV} \lesssim T \lesssim 150 \mathrm{MeV}$.

The color coding in Fig. 1 illustrates the electron fractions $Y_{e}$ reached during the early evolution of a CCSN. The core of the supernova progenitor has almost an equal number of electrons, protons, and neutrons, i.e., $Y_{e} \approx 0.5$. During the collapse, electron-capture reactions lead to a strong neutronization of matter, decreasing $Y_{e}$. The presence of trapped neutrinos, which acquire a finite chemical potential, limits the

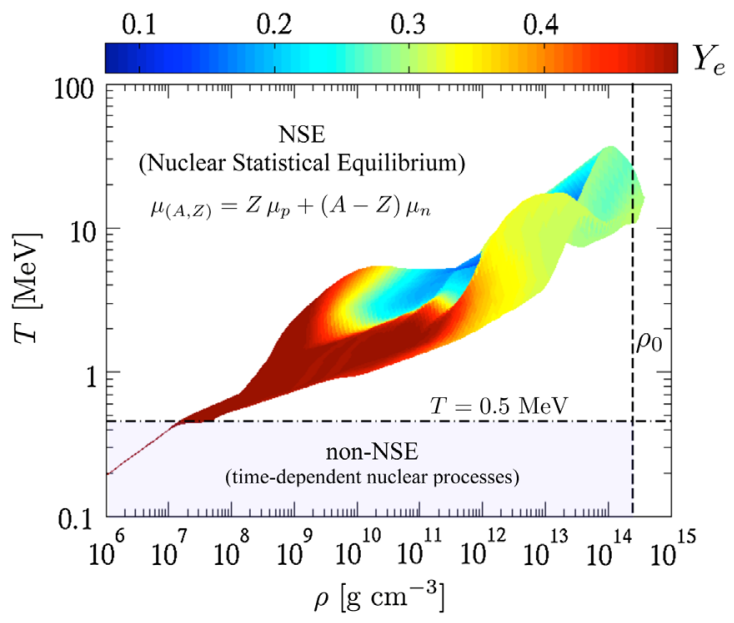

FIG. 1. Temperatures and densities reached during a CCSN simulation within $1 \mathrm{~s}$ post bounce. The color coding shows the electron fraction $Y_{e}$. From T. Fischer.

lowest electron fractions $Y_{e}$ which are reached in the core (Fischer et al., 2011). In its later evolution, the cooling PNS approaches $\beta$ equilibrium with neutrinos freely leaving the system. In the cold, final equilibrium state of a NS, the lowest electron fractions are found to be very close to zero. In some parts of the supernova ejecta $Y_{e}$ can rise to values above 0.5 corresponding to a proton-rich environment. Consequently, the range of a general purpose EoS to be covered is $0<Y_{e} \lesssim 0.6$.

The conditions in NS mergers are quite diverse. In general they depend on the masses of the merging NSs and the EoS and also on the magnetic fields and NS spins. Typical temperatures in the core of a postmerger remnant NS are in the range from 20 to $60 \mathrm{MeV}$ (Bauswein, Janka, and Oechslin, 2010). These temperatures can be well exceeded in the contact layers in the early stage of the merger, where extremely high temperatures up to $150 \mathrm{MeV}$ can occur locally (Bauswein, Janka, and Oechslin, 2010; Rosswog, Piran, and Nakar, 2013). The highest densities in the hot and rotating remnant NS are typically between $2 n_{\text {sat }}$ and $6 n_{\text {sat }}$ (Hotokezaka, Kiuchi et al., 2013). In case the remnant collapses to a $\mathrm{BH}$, similar arguments as for failed $\mathrm{SNe}$ apply: during the collapse much higher densities and correspondingly higher temperatures are reached, but are probably not important dynamically.

The dynamic ejecta of NS mergers originate from the crust and outer core of the merging NSs. Initially, this material has very low $Y_{e}$ in the range from 0.0 to 0.2 (Rosswog, Piran, and Nakar, 2013; Sekiguchi et al., 2015). Depending on the temperatures reached, the degeneracy of electrons is lifted and $Y_{e}$ increases to higher values. In the subsequent evolution, neutrino absorptions also influence $Y_{e}$, resulting in final values in the range of roughly 0.1 to 0.4 (Wanajo et al., 2014; Sekiguchi et al., 2015). See also Foucart et al. (2016) for a comparison of the thermodynamic conditions for different EoS. Figure 2 shows the thermodynamic conditions reached in the remnant in the aftermath of a neutron star merger. For the later ejecta that appear in the form of a neutrino-driven wind, extremely high entropies per baryon 

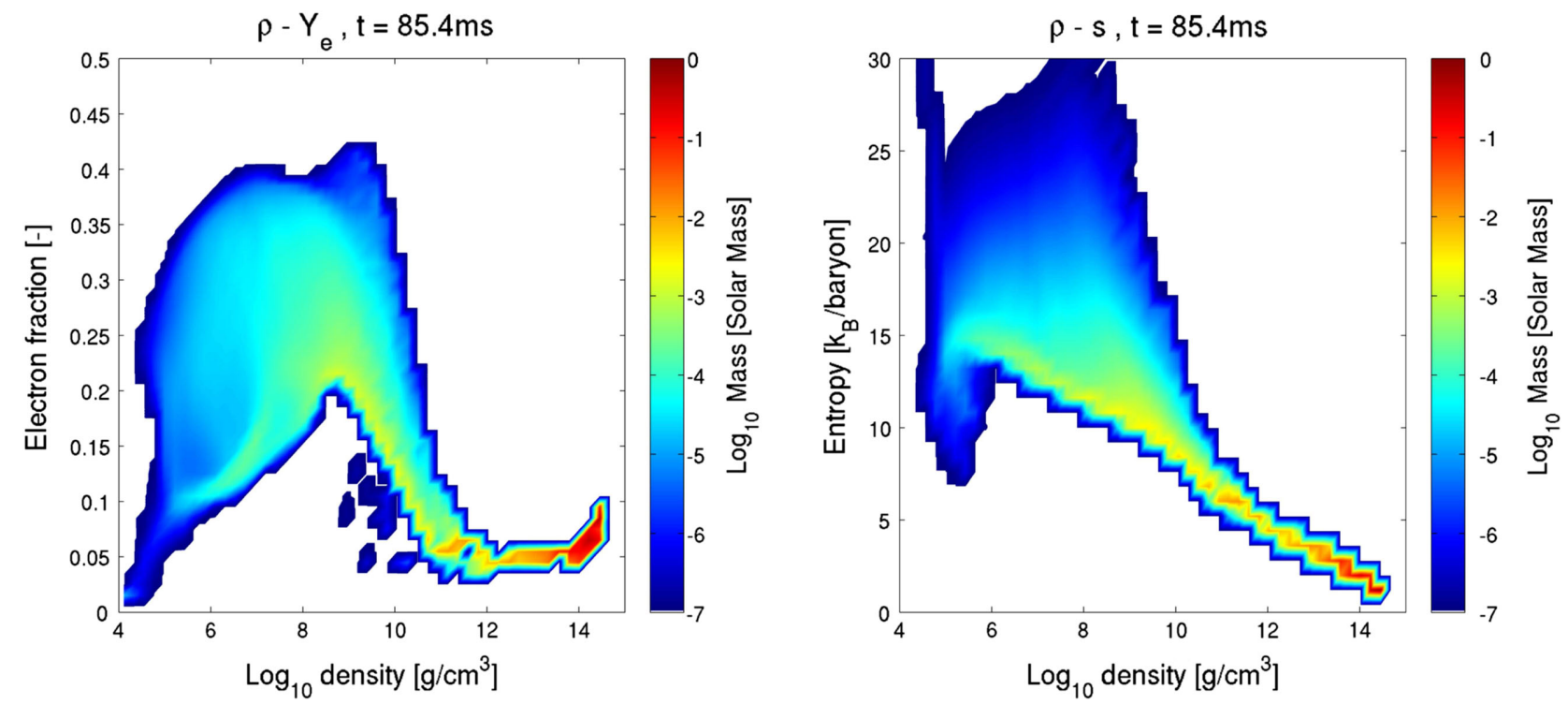

FIG. 2. 2D mass histograms for (left panel) mass density $\rho$ and electron fraction $Y_{e}$ or (right panel) entropy per baryon $s$, of a NS merger remnant at a time of $85.4 \mathrm{~ms}$ after the first contact. The color coding is a measure of the amount of matter experiencing the specific thermodynamical conditions. Adapted from Perego et al., 2014.

above 50 are found (located mostly in the polar regions), whereas most of the matter has entropies below 7, and the entropy tends to decrease with increasing density (Perego et al., 2014; Sekiguchi et al., 2015).

To conclude, we summarize in Table I the overall ranges that have to be covered by a general purpose EoS to describe NS mergers, CCSNe, and cold NSs.

\section{Particle degrees of freedom}

Within these ranges of the thermodynamic variables given, the composition of matter changes dramatically. In cold NSs, heavy nuclei are present in the inner and outer crusts (Chamel and Haensel, 2008). The surface layer of the outer crust is made of ${ }^{56} \mathrm{Fe}$ ions immersed in a sea of electrons. With increasing density, the nuclei become more massive and neutron rich, reaching nuclei of the neutron drip line at the boundary to the inner crust; cf. Sec. V.A.1 for details.

At low densities and finite temperatures, a plasma is expected with a mixture of nuclei, nucleons, and electrons. In the shock-heated matter of CCSNe, light nuclear clusters, such as $\alpha$ particles, deuterons, or tritons, are found to be the dominant baryonic particle degrees of freedom besides nucleons (Sumiyoshi and Röpke, 2008). At densities just below nuclear saturation or sufficiently high temperatures, nuclei dissolve and one is left with strongly interacting matter composed of nucleons and electrons. At high densities and/or

TABLE I. Approximate ranges of temperature, baryon number density, and electron fraction a general purpose EoS has to cover to be able to describe cold NSs, NSs in binary mergers, and CCSNe.

\begin{tabular}{lc}
\hline \hline Quantity & Range \\
\hline Temperature & $0 \mathrm{MeV} \leq T<150 \mathrm{MeV}$ \\
Baryon number density & $10^{-11} \mathrm{fm}^{-3}<n_{B}<10 \mathrm{fm}^{-3}$ \\
Electron fraction & $0<Y_{e}<0.6$ \\
\hline \hline
\end{tabular}

temperatures, additional particle species are expected to occur (Glendenning, 1997; Weber, 2005), such as nuclear resonances, e.g., $\Delta$ baryons (Drago et al., 2014), or mesons, e.g., pions. Also strange degrees of freedom such as hyperons (Glendenning, 1982; Chatterjee and Vidaña, 2016) or kaons can be present. There is the possibility that the mesons form condensates at low temperatures (Glendenning, 1997). Even a transition to deconfined quark matter (QM) is possible at high densities and temperatures. The occurrence of antiparticles is relevant at high temperatures, in particular, for light particle species. Besides electrons, muons are relevant leptonic degrees of freedom, and so are electron-, muon-, and tauflavor neutrinos and antineutrinos. Neutrinos are not necessarily in equilibrium with matter (see Sec. II.B.1). At finite temperatures, thermal photons complete the composition. While leptons and photons can be mostly treated as free gases, this does not hold for hadrons or quarks. Their contribution to the EoS is mainly governed by the strong interaction deeply inside the nonperturbative regime.

\section{FORMAL APPROACHES TO THE DESCRIPTION OF DENSE MATTER}

The theoretical description of strongly interacting matter requires methods which capture the essential thermodynamic properties of the many-body system. The challenges are multifaceted. First, the relevant degrees of freedom have to be identified. Approaches for nuclear matter that are based on nucleons are the predominant choice and may suffice in many cases. It will be necessary to consider other degrees of freedom for certain thermodynamic conditions, e.g., nuclei at low temperatures and densities and hyperons or even quarks at high temperatures and densities. Furthermore, large isospin asymmetries of the system can shift the dominating degrees of freedom to more exotic particles. Second, the interactions between the constituents have to be 
specified. This is a nontrivial task due to the very complex nature of the strong interaction. In addition, the representation depends on the chosen degrees of freedom. In principle, one wants to describe matter directly within the wellfounded theory of QCD. However, there are no $a b$ initio QCD calculations of dense matter available at the thermodynamic conditions that are characteristic for compact stars or CCSNe. Even a derivation of the "true" interaction between nucleons or other strongly interacting hadrons from QCD remains a very complex task despite intensive efforts. Hence, calculations have to rely on model interactions, which are partially constrained by laboratory measurements. Modern theoretical approaches aim at a derivation of input interactions that are systematically improvable with controlled uncertainties.

In the next step, an appropriate method is applied to find the actual state of the system incorporating few- and many-body correlations. In the case of a phase transition, additional thermodynamical considerations have to be applied (see Sec. III.D).

The choice of the degrees of freedom, the choice of the interaction, and the selection of the many-body method are not independent and many different approaches exist. Here we divide them into two categories:

(1) Ab initio many-body methods start from "realistic" few-body interactions (mainly two- and three-nucleon forces), i.e., interactions that are fitted to observables in nucleon-nucleon scattering in vacuum and properties of bound few-nucleon systems. The many-body problem is then treated using different techniques, e.g., Green's function methods, (Dirac)-Brueckner-HartreeFock calculations, coupled cluster, variational, and Monte Carlo methods; see Sec. III.B.1. Some of these many-body methods are limited by technical problems, such as the Monte Carlo methods; others introduce approximations, such as, for example, Brueckner-type approaches, that consider only a subclass of all possible diagrams.

(2) Phenomenological approaches use effective interactions that often have a more simple structure than realistic interactions used in $a b$ initio approaches. They depend on a small number of parameters, usually of the order of 10 to 15 , which are fitted, in the ideal case, to different properties of several nuclei all over the chart of nuclei and nuclear matter properties. Typical representatives of these effective interactions are the Skyrme and Gogny forces in nonrelativistic calculations and meson-exchange forces in relativistic mean-field (RMF) models; see Sec. III.B.2. Nowadays, these phenomenological approaches are interpreted in terms of energy density functional (EDF) theory. Applying simple manybody methods, mostly on the mean-field (MF) level, results already in a rather precise description of nuclei and nuclear matter. The extrapolation to exotic conditions has to be considered with caution; nevertheless, phenomenological approaches are the most widely used methods to construct EoSs for astrophysical applications.

\section{A. Basic few-body interactions}

Basic few-body interactions are the starting point of any calculation of dense matter with ab initio many-body methods. The two-body interaction is largely dominant, but interactions beyond the two-body level become important in matter at high densities. For example, it is well known that the nuclear three-body force is essential to reproduce the saturation properties of nuclear matter. Forces among four or more nucleons are difficult to construct and can be neglected in many circumstances. Realistic two- and three-body forces between nucleons and hyperons, as discussed in this section, should not be confused with effective temperature and density-dependent interactions used in phenomenological models (see Sec. III.B.2). A more detailed survey of modern theories for nuclear forces can be found in the review of Epelbaum, Hammer, and Meissner (2009).

Historically, Yukawa (1955) proposed the first model of the $N N$ interaction based on the exchange of a massive particle, the pion. His model successfully explained the range of the nuclear interaction. Since then, many phenomenological models have been developed either based on Yukawa's idea of meson exchange or by constructing potentials with appropriate operator structure. With the advent of QCD as the theory of the strong interaction in the 1970s, phenomenological quark models became very fashionable, describing baryons as quark clusters. They, however, suffer from the missing confinement and connection with QCD. Only recently, with chiral effective field theories ( $\chi$ EFTs) and with lattice gauge theory, considerable progress has been achieved to link baryonic few-body forces to QCD.

\section{Experimental data}

Any theoretical model for baryonic forces can be tested by comparing predictions to experimental data. This concerns scattering and the structure of light nuclei and hypernuclei. In the nuclear sector, many thousands of high-precision data points are available. A complete partial wave analysis of nucleon-nucleon $(N N)$ scattering data can be performed; see Stoks et al. (1993), Arndt, Strakovsky, and Workman (1994), Arndt et al. (2007), and Navarro Pérez, Amaro, and Ruiz Arriola (2013) and the corresponding online databases. Deuteron properties, among others its binding energy and electric quadrupole moment, are an important input for $N N$ forces. Owing to the large amount of data, today's $N N$ interactions, phenomenological or based on effective field theories, have reached a very high degree of precision.

The binding energies of other light nuclei and nucleondeuteron scattering data cannot be described satisfactorily on the basis of a two-nucleon interaction and provide thus valuable information on the nuclear three-body force; see Kalantar-Nayestanaki et al. (2012) for a review. Recently, properties of very neutron-rich nuclei have attracted attention since they provide additional constraints on the three-body force; see, e.g., Wienholtz et al. (2013).

For the hyperonic sector data are scarce; see Gal, Hungerford, and Millener (2016) for a detailed review of strangeness in nuclear physics. Hyperon-nucleon $(Y N)$ scattering experiments are difficult to perform because hyperons 
have very short lifetimes of the order of $10^{-10} \mathrm{~s}$. Data on hyperon-hyperon $(Y Y)$ scattering are not available. In the fitting procedure for the parameters of hyperonic two-body forces, in general only 35 data points from the 1960s (Engelmann et al., 1966; Alexander et al., 1968; SechiZorn et al., 1968; Eisele et al., 1971) for low-energy total cross sections in reactions involving $\Lambda$ and $\Sigma$ hyperons are included. First low-energy data on $\Xi^{-} p$ elastic and $\Xi^{-} p \rightarrow$ $\Lambda \Lambda$ scattering have been obtained at KEK (Ahn et al., 2006).

In addition to scattering experiments, hypernuclear spectroscopy can provide valuable information. Since the first events recorded by Danysz and Pniewski (1953a, 1953b), many hypernuclei have been produced. These are mainly single- $\Lambda$ hypernuclei; see, e.g., the reviews by Hashimoto and Tamura (2006) and Gal, Hungerford, and Millener (2016). Some events with double- $\Lambda$ hypernuclei have been detected; see Aoki et al. (1991) and Nakazawa (2010). The absence of $\Sigma$-bound states, except for an $s$-wave $\Sigma$ bound state in ${ }_{\Sigma}^{4} \mathrm{He}$ (Nagae et al., 1998), indicates a repulsive $\Sigma N$ interaction (Bart et al., 1999; Saha et al., 2004; Kohno et al., 2006). Only a few events for $\Xi$ hypernuclei have been observed up to now (Aoki et al., 1995; Fukuda et al., 1998; Khaustov et al., 2000). Considerable experimental efforts are underway to improve hypernuclear data; see, e.g., Agnello et al. (2012) and Sugimura et al. (2014). Three-body forces are not yet well explored for hyperons; see, however, the recent work by Lonardoni, Gandolfi, and Pederiva (2013). Hyperonic singleparticle potentials in symmetric nuclear matter are often used to determine the effective hyperon-nucleon interactions in phenomenological models; see, e.g., the discussions by Ellis, Kapusta, and Olive (1991), Glendenning and Moszkowski (1991), Schaffner et al. (1994), Balberg and Gal (1997), Glendenning (1997), Vidaña et al. (2001), and Oertel, Fantina, and Novak (2012).

\section{Phenomenological forces}

Approaches to obtain phenomenological forces can be divided into two main categories: models based on meson exchange and potential models.

\section{a. Meson-exchange models}

Meson-exchange models follow the original idea of Yukawa in which the $N N$ interaction is mediated by meson exchange. Additional mesons have been added to capture the complex dependence of the nuclear interaction on spin, isospin, and spatial coordinates. Some models have been extended to include strange mesons in order to describe the $Y N$ and in some cases the $Y Y$ interaction. The general idea is that the pion, as the lightest particle, describes the long-range attractive part of the interaction and that scalar mesons are responsible for the intermediate-range attraction whereas vector mesons govern the short-range repulsive contribution. The so-called $\sigma$ meson in the scalar-isoscalar channel often represents the midrange attraction; however, its status as a particle is very ambiguous. Many models use instead correlated and uncorrelated two-pion exchange to describe the intermediate range $N N$ interaction; see, e.g., Machleidt and Li (1994) and Donoghue (2006) for discussions. The various models differ mainly in the mesonic content, the treatment of two-meson exchange, and approximations made in order to obtain practically applicable potentials from the basic amplitudes, for instance, the form factors used at the meson-baryon interaction vertices. From a phenomenological point of view, the latter are introduced to account for the substructure of baryons. They serve as regulators in solving the scattering equation in order to avoid any divergent contributions.

Next we mention some well-known models that satisfactorily describe $N N$ scattering data and the deuteron. The classical versions of the Nijmegen interaction for the $N N$ system (Nagels, Rijken, and de Swart, 1977, 1978) are based on the one-meson-exchange picture. They were extended to include $Y N$ and $Y Y$ interactions (Maessen, Rijken, and de Swart, 1989; Rijken, Stoks, and Yamamoto, 1999) as well as additional one- and two-meson exchanges (Rijken, 2006; Rijken and Yamamoto, 2006; Nagels, Rijken, and Yamamoto, 2014). The Paris $N N$ potential (Cottingham et al., 1973; Lacombe et al., 1980) uses an ad hoc parametrization at very short distances arguing that the meson-exchange picture is no longer valid there due to the substructure of nucleons. The Bonn potential (Machleidt, Holinde, and Elster, 1987; Machleidt, 2001) for the $N N$ interaction is given in relativistic form in momentum space to avoid the local approximation of nonrelativistic models, as, e.g., in the Nijmegen potentials. The Jülich group has extended the Bonn model to the $Y N$ interaction (Holzenkamp, Holinde, and Speth, 1989; Haidenbauer and Meissner, 2005).

\section{b. Potential models}

In addition to the well-established long-range one-pion exchange, potential models adopt a sum of local operators, where the essential ones are central, tensor, and spin-orbit terms. The parameters are fitted to deuteron properties and $N N$-scattering data. The Urbana (Lagaris and Pandharipande, 1981) and Argonne potentials (Wiringa, Smith, and Ainsworth, 1984; Wiringa, Stoks, and Schiavilla, 1995) are examples of such high-quality potential models. The latest version of the Argonne potential (Wiringa, Stoks, and Schiavilla, 1995), called $v_{18}$, not only contains isoscalar operators but includes an electromagnetic part and isovector operators such that the charge dependence of the $N N$ force is successfully described. Also, some models with $\Lambda$ hyperons are available, but they are much less sophisticated due to the small amount of hyperonic data; see, e.g., Bodmer, Usmani, and Carlson (1984).

Considering only two-nucleon interactions, it is well known that light nuclei, in particular, the triton ${ }^{3} \mathrm{H}$, are underbound and the saturation density of nuclear matter is overestimated. This shows the importance of many-body forces to correctly describe nuclear systems. A major contribution to the threenucleon force is the two-step pion exchange between two nucleons via a third nucleon that can be excited, e.g., to a $\Delta$ baryon (Fujita and Miyazawa, 1957). This feature is incorporated for instance in the Tucson-Melbourne model (Coon and Glöckle, 1981; Friar, Huber, and van Kolck, 1999; Coon and Han, 2001). Such an interaction is attractive and helps to solve the underbinding problem in light nuclei, whereas it worsens nuclear matter saturation properties. Therefore the Urbana group proposed a series of 
phenomenological three-nucleon forces, adding to the attractive two-pion exchange contribution a parametrized repulsive part (Carlson, Pandharipande, and Wiringa, 1983; Pudliner et al., 1995; Pieper et al., 2001; Pieper, 2008). The latter interaction is adjusted to the properties of light nuclei. The problem of such a procedure is that the three-body force is not independent of the two-body force employed in the fits. More recently, consistent two- and three-nucleon forces have been derived within $\chi$ EFT; see the next section.

\section{Interactions from chiral effective field theory and lattice QCD}

Since the seminal papers by Weinberg $(1990,1991)$ many efforts have been devoted to the derivation of nuclear forces from a $\chi \mathrm{EFT}$. Within a chiral theory pions emerge naturally as the relevant degrees of freedom at low energies to describe the interaction of nucleons since they appear as Goldstone bosons of the theory if the chiral symmetry of QCD is spontaneously broken. The systematic framework of effective field theories allows one to establish a classification of different contributions to the interaction and to make the link with QCD. The starting point is the most general effective chiral Lagrangian that respects the required symmetries. It is expanded in powers of a small quantity $p \sim\left(m_{\pi} / \Lambda_{\chi},|\vec{k}| / \Lambda_{\chi}\right)$, where $m_{\pi}$ denotes the pion mass, $\vec{k}$ is a (soft) external momentum, and $\Lambda_{\chi} \sim$ $1 \mathrm{GeV}$ specifies the scale of chiral symmetry breaking. In addition to dynamical pion contributions, nucleonic contact operators appear at each order. They contain the unresolved short-range physics. Their strength is controlled by so-called low-energy constants (LECs) that are fitted to experimental data. Pionless effective theories are applicable at very low energies; see Bedaque and van Kolck (2002) for a review.

Although the details of the power counting scheme are not yet completely settled [see, e.g., Pavón Valderrama and Phillips (2015) and references therein], chiral nuclear forces work out well. In particular, before the advent of nuclear interactions from $\chi$ EFTs, no consistent model of three-body and higher many-body nuclear forces existed. In addition to the incorporation of symmetries from QCD, the advantage of $\chi$ EFT approaches is the possibility to extend the interactions in a consistent way to three- and many-nucleon systems. Comprehensive reviews can be found in Epelbaum, Hammer, and Meissner (2009) and Machleidt and Entem (2011). For recent high-quality chiral potentials, see Navarro Pérez, Amaro, and Arriola (2015) and Piarulli et al. (2015), which includes $\Delta$ resonances. The $\chi$ EFT approach has been extended to include strangeness and interactions of the full baryon octet; see Polinder, Haidenbauer, and Meißner (2006) and Haidenbauer et al. (2007, and 2013). In this case, not all LECs can be determined by experiment due to the lack of relevant data in the hyperonic sector. Instead they have partly been fixed by flavor $S U(3)$ symmetry.

Another promising possibility to relate nuclear forces to QCD is lattice QCD. In principle, it is a tool to calculate hadron properties directly from the QCD Lagrangian with Monte Carlo methods on a discretized Euclidian spacetime. It is, however, extremely expensive in the numerical application even with sophisticated state-of-the-art algorithms on high performance computers. For the moment, simulations can be carried out only with large quark masses and the extrapolation to physical masses is difficult. In addition, the lattice spacing has to be fine enough and the volume large enough to avoid computational artifacts. Recent substantial efforts (Beane et al., 2011; Aoki et al., 2012) give hope for future highprecision predictions. This could be interesting, in particular, for channels where only few experimental data are available, e.g., the hyperon-nucleon interaction; see, e.g., Beane et al. (2007, 2012) and Inoue et al. (2010).

\section{Renormalization group methods and evolved potentials}

The strongly repulsive core of two-body baryonic interactions renders multibaryon systems nonperturbative. Thus correlations become extremely important but are difficult to treat with many-body methods. The repulsive core, although a distinct feature of baryonic forces, is not directly affecting low-energy observables. With renormalization group (RG) techniques, the high-momentum part of the interaction related to the repulsive core can be "integrated out" via a continuous change in resolution by applying suitable unitary transformations. In this way, the high-momentum part decouples from the low-momentum part and three- and many-body forces emerge automatically from a pure two-body force. During the evolution, all generated interactions are "phase-shift equivalent" and low-momentum observables are preserved. Thus the description of scattering data remains as precise as for the original interaction. The obtained RG-evolved potentials are much more perturbative than nonevolved ones and therefore simplify the baryonic many-body problem. In connection with many-body perturbation theory (MBPT), i.e., a perturbative expansion around the Hartree-Fock (HF) solution, RGevolved interactions became a great success for nuclear systems; see Bogner, Furnstahl, and Schwenk (2010) and Furnstahl and Hebeler (2013) for recent reviews on the subject.

Even though all high-precision "bare" nuclear forces are rather different, almost unique RG-evolved potentials emerge at low momenta (Schwenk, 2005), often denoted as $V_{\text {low- } k}$. Schaefer et al. (2006) and Wagner et al. (2006) applied the same techniques to nucleon-hyperon interactions. It turned out that the resulting low-momentum interactions are different from each other because the bare potentials are much less constrained. Hence, it is not surprising that there is a large spread in the results if they are applied to dense matter with hyperons (Đapo, Schaefer, and Wambach, 2008, and 2010). This shows the lack of relevant experimental data concerning the hyperon-nucleon and hyperon-hyperon interactions.

\section{B. Many-body methods for homogeneous matter}

The first step in studying strongly interacting matter is often the investigation of homogeneous matter at vanishing temperature where almost all methods discussed later can be applied. Even if the basic few-body interactions were exactly known, the theoretical modeling is not a trivial task since any naive perturbative expansion is likely to fail.

The most simple method to treat the many-body problem beyond the perturbative level is the HF approximation; see Sec. III.B.2 and Fetter and Walecka (1971), Ring and Schuck 
(1980), and Greiner and Maruhn (1996) for more details. The idea is that each particle moves in a single-particle potential, the "mean field," generated by the average interaction with all other particles. In practice, the many-body wave function is approximated as an antisymmetrized product of singleparticle wave functions, which are determined selfconsistently. Although generally successful in atomic physics and in chemistry, in a nuclear system a HF calculation starting from conventional two-body interactions fails to reproduce known properties of nuclear matter. In addition, the results are sensitive to the modeling of the short-range repulsive core of the two-body interaction which is not fixed uniquely by scattering data. This can be understood since the HF approximation neglects any short-range correlations between the particles which arise from their mutual interaction.

Two ways out of this problem are currently applied: either correlations are explicitly included within the many-body approach or, instead of realistic few-body interactions, an effective, usually medium dependent, interaction is used within a HF approach.

In Sec. III.B.1, we discuss different theoretical ab initio frameworks to include correlations in a strongly interacting many-body system. Interesting attempts to compare in a quantitative way different $a b$ initio many-body methods can be found, e.g., in Baldo and Maieron (2004), Bombaci et al. (2005), and Baldo et al. (2012). Section III.B.2 is devoted to models with different types of phenomenological effective interactions. Apart from the textbooks, there exist many excellent reviews on the different standard many-body methods; see, e.g., Müther and Polls (2000), Baldo and Burgio (2012), and Carlson et al. (2015). Therefore we do not aim to give a comprehensive and complete overview, but present only the general ideas.

\section{1. $A b$ initio methods}

\section{a. Self-consistent Green's function}

The idea of the self-consistent Green's function (SCGF) method is that the system's energy can be calculated conveniently from the single-particle Green's function $\mathcal{G}$. It describes the propagation of a single-particle state $\psi$ from time $t$ and position $\vec{x}$ to $t^{\prime}$ and $\vec{x}^{\prime}$ as

$$
\psi\left(t^{\prime}, \vec{x}^{\prime}\right)=\int d^{4} x \mathcal{G}\left(t^{\prime}, \vec{x}^{\prime}, t, \vec{x}\right) \psi(t, \vec{x}) .
$$

The level of approximation in the SCGF method is controlled by the approximations made in order to determine the singleparticle Green's function. A thorough definition can be found in any textbook on quantum theory at finite density and temperature; see, e.g., Fetter and Walecka (1971). For a noninteracting homogeneous system at zero temperature the Green's function can be written in momentum space $a^{2}$

\footnotetext{
${ }^{2}$ For simplicity we assume nonrelativistic kinematics. A relativistic treatment does not change the general reasoning. We further consider the zero temperature limit. Finite temperature is easily included in the formalism; see, e.g., Fetter and Walecka (1971) and Frick (2004).
}

$$
\mathcal{G}^{0}(\vec{k}, \omega)=\frac{\theta\left(|\vec{k}|-k_{F}\right)}{\omega-E^{0}(\vec{k})+i \eta}+\frac{\theta\left(k_{F}-|\vec{k}|\right)}{\omega-E^{0}(\vec{k})-i \eta},
$$

where $k_{F}$ denotes the Fermi momentum and $E^{0}(\vec{k})=\vec{k}^{2} /(2 m)$ denotes the noninteracting single-particle energy of a particle with mass $m$. Any indices related to further quantum numbers of the particle are suppressed for clarity. The first term on the right-hand side (rhs) of Eq. (4) describes the propagation of a state outside the Fermi sea, a particle, and the second term a state inside the Fermi sea. Since per definition all states in the Fermi sea are filled, it can propagate inside the Fermi sea only as a hole, i.e., a particle removed from the Fermi sea.

The energy density of the system can be straightforwardly calculated from the trace of the single-particle Green's function. For the noninteracting system the well-known expression for an ideal Fermi gas is obtained. Of course, a dense baryonic system cannot be described as a Fermi gas of noninteracting particles. Thus the full interacting singleparticle Green's function $\mathcal{G}$ has to be determined. A calculation from a perturbative series in the interaction potential is not viable in view of the strong baryonic interaction. At this point self-consistency is introduced in the form of Dyson's equation, which schematically can be written as

$$
\mathcal{G}=\mathcal{G}^{0}+\mathcal{G}^{0} \Sigma \mathcal{G}
$$

with the one-particle irreducible ${ }^{3}$ self-energy $\Sigma$ which itself is determined by the interaction.

Retaining the lowest-order diagrams in the interaction, the HF approximation can be derived with the SCGF formalism. Formally this means that in the HF approximation the $N$-particle Green's functions are (antisymmetrized) products of single-particle Green's functions. When going beyond the $\mathrm{HF}$ approximation, the dominant effect should be multiple scattering processes with two participating baryons. Under this assumption the so-called "ladder approximation" is obtained. The name originates from a diagrammatic representation of this approximation for the single-particle Green's function depicted in Fig. 3. In practice, the complete twoparticle $T$ matrix is introduced, describing an effective twoparticle interaction upon summing up all "ladders"; see Fig. 3. Note that the ladder approximation includes the two contributions leading to the HF approximation. The equation for the $T$ matrix can be written as

$$
\langle 12|T| 34\rangle=\langle 12|V| 34\rangle+\sum_{n n^{\prime}}\left\langle 12|V| n n^{\prime}\right\rangle \mathcal{G}_{n} \mathcal{G}_{n^{\prime}}\left\langle n n^{\prime}|T| 34\right\rangle,
$$

where $V$ represents the bare two-body interaction and a summation over intermediate states has to be performed.

In the intermediate states of the $T$ matrix there can be no propagation of a particle and a hole state. In the ladder

\footnotetext{
${ }^{3}$ One-particle irreducible means that the diagrams cannot be disconnected by cutting a fermion line. It is obvious that the selfconsistent resummation via Dyson's equation automatically generates all reducible terms.
} 


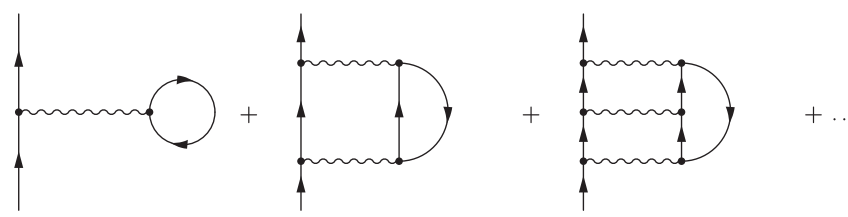

FIG. 3. Feynman diagrams illustrating the lowest-order contributions to the self-energy in Dyson's equation (5) for the singleparticle Green's function in the ladder approximation (without exchange contributions). Solid lines represent fermion propagators and wavy lines an interaction.

approximation the self-consistent Green's function method thus sums up particle-particle and hole-hole ladders to all orders. Physically the ladder diagrams take into account multiple scattering of particles and holes and can therefore, in contrast to the HF approximation, describe the effect that the strong short-range repulsion disfavors states when two particles come very close to each other. Reviews on the applications of the method to nuclear problems can be found in Müther and Polls (2000) and Dickhoff and Barbieri (2004). The results for the EoS improve substantially in comparison to the HF approximation.

The choice of the contributions included in the summation (ladder, ring, parquet) has been more or less intuitive and was justified by the results. It is not clear how the method can be systematically extended in order to improve the results achieving self-consistency and avoiding double counting. So far three-nucleon forces were not included in the ladder approximation although their importance in nuclear systems is well known. A method to include them in the SCGF formalism was developed recently by Carbone et al. (2013).

Another point is that low-temperature nuclear (and baryonic) matter is unstable with respect to the formation of a superfluid or superconducting state. This is the well-known Cooper instability (Cooper, 1956): a fermionic many-body system with an attractive interaction tends to form pairs at the Fermi surface. The instability shows up as a pole in the $T$ matrix when the temperature falls below the critical temperature $T_{c}$ for the transition to the superfluid or superconducting state; see, e.g., Thouless (1960), Schmidt, Röpke, and Schulz (1990), Alm et al. (1993), and Stein et al. (1995). Formally, the approach can be extended to include the possibility of superfluidity and superconductivity by introducing anomalous Green's functions describing pair formation. However, practical SCGF calculations in the ladder approximation are numerically already very demanding since the full energy dependence of the intermediate states has to be accounted for. Therefore actual calculations are often performed at temperatures above $T_{c}$ and extrapolated to zero temperature; see, e.g., Frick (2004).

\section{b. Brueckner-Hartree-Fock}

The Brueckner-Hartree-Fock (BHF) approximation is a widely used microscopic many-body method developed by Brueckner, Bethe, and others in the 1950s. Numerically it is less involved than the SCGF discussed previously. In a general framework it can be derived from the Brueckner-BetheGoldstone hole-line expansion, truncated at the two-hole-line level for the evaluation of the ground-state energy. A "hole line" has to be considered as the propagation of a hole. This series can be roughly understood as an expansion in density (Fetter and Walecka, 1971). At low densities the contributions with an increasing number of hole lines should be suppressed, thus ensuring good convergence. It is generally assumed that the $n$-hole-line contributions contain the dominant part of the $n$-body correlations. Detailed and pedagogical introductions can be found in Day (1967), Fetter and Walecka (1971), and Baldo and Burgio (2001, 2012).

The BHF method can be obtained from the SCGF approach in the ladder approximation after some simplifications. The first one is to neglect the hole-hole contributions. The second one is to approximate the full self-energy of the intermediate states by a quasiparticle approximation. The equation for the $T$ matrix, Eq. (6), then becomes an equation for the Brueckner $G$ matrix by replacing $T$ with $G$, except that the product of the two single-particle Green's functions in the intermediate states is approximated by

$$
\mathcal{G}_{n} \mathcal{G}_{n^{\prime}} \rightarrow \frac{\mathcal{P}\left(n, n^{\prime}\right)}{\omega-E_{n}-E_{n^{\prime}}+i \eta},
$$

where the Pauli operator $\mathcal{P}$ is nonzero only if both states lie outside the Fermi sea, i.e., they correspond to two particle states. Note that the denominator does not contain the full selfenergy. In the quasiparticle approximation it has the form of the noninteracting system.

The single-particle energies $E$ are determined selfconsistently from the $G$ matrix in the following way (written in momentum space):

$$
\begin{gathered}
E(\vec{k})=\frac{\vec{k}^{2}}{2 m}+U(\vec{k}), \\
U(\vec{k})=\sum_{\left|\vec{k}^{\prime}\right|<k_{F}}\left\langle k k^{\prime}\left|G\left(E(\vec{k})+E\left(\overrightarrow{k^{\prime}}\right)\right)\right| k k^{\prime}\right\rangle_{A},
\end{gathered}
$$

where the subscript $A$ indicates that the matrix element has to be antisymmetrized.

The BHF method is not fully self-consistent. There remains some freedom in determining the single-particle energy $E(\vec{k})$; see Baldo and Burgio (2001) for a discussion. It was shown by Baldo et al. (2001) that with a proper choice the three-hole line corrections to the energy are small, indicating good convergence of the series. The main problem is that the BHF method violates the Hugenholtz-van Hove theorem (Hugenholtz and van Hove, 1958) and, hence, is thermodynamically inconsistent. This theorem states that the single-particle energy at the Fermi surface should equal the chemical potential. Numerically the differences are of the order of 10-20 MeV at saturation density (Bożek and Czerski, 2001).

In the BHF approximation, the description of nuclear matter is improved substantially as compared with the HF calculations. However, the saturation properties are not satisfactorily reproduced with only two-body forces. It is generally believed that three-body forces are needed. In nonrelativistic BHF calculations they can be explicitly included; see, e.g., Lejeune, Lombardo, and Zuo (2000) and Zuo et al. (2002a, 
2002b). So far, there is no general consensus on how to improve BHF calculations systematically such that uncertainties are under control.

It is possible to extend the nonrelativistic BHF formalism in order to treat baryons in a special relativistic way. This DiracBrueckner-Hartree-Fock (DBHF) approach (Brockmann and Machleidt, 1984; Horowitz and Serot, 1987; Ter Haar and Malfliet, 1987; Brockmann and Machleidt, 1990; Sammarruca, 2010) is computationally more involved than nonrelativistic BHF calculations and some ambiguities exist concerning the representation of the in-medium $G$ matrix in terms of Lorentz invariants; see, e.g., the discussion by GrossBoelting, Fuchs, and Faessler (1999). However, the main advantage is that an additional repulsion at high densities is obtained since part of the three-body interaction is automatically generated (Brown, 1987). Relativistic DBHF approaches also avoid the problem of nonrelativistic BHF calculations which can result in a superluminal speed of sound at the high central densities of massive NSs.

\section{c. Methods derived from the variational principle}

The Ritz-Raleigh variational principle is the basis for variational approaches to the many-body problem. It ensures that the trial ground-state energy

$$
E_{\text {trial }}=\frac{\left\langle\Psi_{\text {trial }}|H| \Psi_{\text {trial }}\right\rangle}{\left\langle\Psi_{\text {trial }} \mid \Psi_{\text {trial }}\right\rangle},
$$

calculated from the system's Hamiltonian $H$ with a trial manybody wave function $\Psi_{\text {trial }}$, gives an upper bound for the true ground-state energy of the system. Correlations can be embodied in the trial wave function, given for the $A$-baryon system by

$$
\Psi_{\text {trial }}(1, \ldots, A)=F(1, \ldots, A) \Phi_{\mathrm{MF}}(1, \ldots, A) .
$$

The operator $F$ is intended to transform the uncorrelated wave function $\Phi_{\mathrm{MF}}(1, \ldots, A)$ to the correlated one. $\Phi_{\mathrm{MF}}$ is an antisymmetrized product (a Slater determinant) of singleparticle wave functions. In practice, an ansatz is chosen for the trial wave function and its parameters are varied in order to minimize $E_{\text {trial }}$. Once the trial wave function is determined, expectation values of other operators can be evaluated.

The idea of the variational method is very simple and appealing. The difficulty resides, however, in the details, namely, the numerical evaluation of the different expectation values. The first point is the choice of the interaction Hamiltonian. As it stands, the method is conceived for treating a local nonrelativistic potential. ${ }^{4}$ Thus, some of the realistic potentials discussed in Sec. III.A, in particular, those involving energy-dependent meson exchange, cannot be used within this approach. There are, however, potentials which have been designed specifically for variational methods. The most prominent example is the series of Argonne $N N$ forces (see Sec. III.A.2.b). Most variational calculations include a threebody force in the nuclear Hamiltonian. Together with the

\footnotetext{
${ }^{4}$ See Walhout et al. (1996) for an attempt to generalize this method to relativistic systems within the path integral formalism.
}

Argonne forces, the Urbana three-body forces are usually applied. Further, Gezerlis et al. (2013, 2014) developed a local version of nuclear interactions for quantum Monte Carlo calculations from $\chi \mathrm{EFT}$ which in principle is applicable.

The central task is to find a suitable ansatz for the correlation operator $F$. At sufficiently low densities, twobody correlations should be dominant in nuclear systems. This assumption represents, for instance, the basis for the ladder approximation discussed in Sec. III.B.1.a. Within the variational methods this assumption leads to an ansatz for the two-body correlation operator $F_{2}$ which contains essentially the same operational structure as the two-body interaction (Fantoni and Fabrocini, 1998; Müther and Polls, 2000; Carlson et al., 2015). Thus, it is written as a sum of twobody operators, incorporating the nuclear spin-isospin dependence, multiplied by radial correlation functions $f^{(m)}(r)$ :

$$
F_{2}(i, j)=\sum_{m} f^{(m)}\left(r_{i j}\right) \mathcal{O}^{(m)}(i, j)
$$

The $f^{(m)}(r)$ are then determined by minimizing $E_{\text {trial }}$. In practice, this is done employing different techniques.

In the nuclear context, Fermi-hypernetted-chain (Fantoni and Rosati, 1975; Pandharipande and Wiringa, 1979) calculations have proven to be efficient. We note that up to now it is impossible to include the complete operator structure of the most sophisticated Argonne potentials in the correlation operators $F$. The spin-orbit correlation, in particular, cannot be treated on the same footing, since it cannot be chained.

In coupled cluster theory, which is based on ideas of Coester (1958) and Coester and Kümmel (1960), the correlation operator $F$ is represented in an exponential form $F=$ $\exp (\hat{T})$ with the cluster operator

$$
\hat{T}=\sum_{m=1}^{A} \hat{T}_{m}
$$

that is a sum of $m$-particle $m$-hole excitation operators $\hat{T}_{m}$. The method, which is nonvariational in practice, is utilized with great success in quantum chemistry (Bartlett and Musial, 2007) and nuclear structure calculations (Hagen et al., 2007, 2010, 2012). Early applications to nuclear matter can be found in Kümmel, Lührmann, and Zabolitzky (1978) and Day and Zabolitzky (1981). Nowadays, optimized chiral nucleonnucleon interactions are implemented (Baardsen et al., 2013; Hagen et al., 2014).

The variational ground-state energy represents only an upper bound on the exact ground-state energy, and the deviation depends on the choice of the trial wave function. The method of correlated basis functions (CBF) allows one to improve on the variational ground state (Fantoni and Fabrocini, 1998). The idea is to add up second-order perturbative corrections to the ground-state energy calculated with correlated basis functions. The latter are determined by the variational calculation from model basis functions. Taking only $\Phi_{\mathrm{MF}}$ as a model basis function, the usual variational calculation would be recovered. Within CBF, other basis 
functions are added, containing already some particle-hole excitations on the initial wave function $\Phi_{\mathrm{MF}}$.

Another widely used method in nuclear physics to evaluate expectation values is the variational Monte Carlo approach; see the reviews by Guardiola (1998) and Carlson et al. (2015). Sophisticated calculations with this technique have been performed for light nuclei, including two-body correlations, such as given in Eq. (12), and triplet correlations; see, e.g., Wiringa et al. (2014). Since the computational effort increases very rapidly with the number of nucleons, the EoS of homogeneous nuclear matter, however, is extremely difficult to obtain.

\section{d. Quantum Monte Carlo methods}

Advancing computer technology has allowed for rapid progress in the application of Monte Carlo methods to nuclear systems in recent years. In addition to the variational Monte Carlo approach discussed in the previous section, the ground-state wave function and energy can be determined by evolving the many-body Schrödinger equation in imaginary time. Monte Carlo sampling is thereby used to evaluate the paths. In nuclear physics these methods suffer, however, from the fermion sign problem and different approximations are employed; for comprehensive reviews, see Guardiola (1998), Carlson, Gandolfi, and Gezerlis (2012), and Carlson et al. (2015).

The Green's function Monte Carlo (GFMC) method (Carlson, 1987, 1988) gives accurate results for light nuclei, but due to the nuclear spin and isospin degrees of freedom, computing time increases exponentially with the number of particles. Up to now, the largest systems treated are ${ }^{12} \mathrm{C}$ and a system of 16 neutrons. The auxiliary field quantum Monte Carlo (AFQMC) approach (Schmidt and Fantoni, 1999) introduces auxiliary fields by Hubbard-Stratonovich transformations to sample the spin-isospin states. This efficient sampling allows for treating larger systems, with more than 100 nucleons. Finite-size effects are expected to be small and AFQMC calculations have been applied in the last years to homogeneous matter, in particular, neutron matter, but also to symmetric matter and nuclear matter with hyperons (see Sec. IV.B).

In spite of the recent progress, it is still not possible to perform GFMC and AFQMC calculations with the full Argonne $v_{18}$ potential since some of the terms, again related to the spin-orbit structure, induce very large statistical errors. Simplified potentials have been developed (Pudliner et al., 1997; Wiringa and Pieper, 2002) containing less operators with readjusted parameters. Besides these two-body interactions, the Urbana three-body potentials are used. Recently, a local chiral potential was developed (Gezerlis et al., 2013) which is well suited for quantum Monte Carlo techniques.

\section{e. Chiral effective field theory}

$\chi$ EFT is very successful in describing nuclear forces and has allowed one to establish a link between the underlying theory of QCD and nuclear physics (see Sec. III.A.3). Except for very light nuclei, where direct numerical solutions of the Schrödinger equation are possible, these chiral forces are generally employed within standard many-body techniques to address heavier nuclei or homogeneous nuclear matter. In recent years some effort has been devoted to an alternative approach for homogeneous matter, namely, extending the idea of chiral perturbation theory directly to nuclear matter calculations, i.e., developing an effective field theory (EFT) for nuclear matter. Similar to nuclear forces in vacuum, pions and nucleons are treated as explicit degrees of freedom and short-range dynamics is comprised in local contact terms. The advantage of such an EFT is that it establishes a power counting which allows one to select at a given order the relevant ones among the infinite number of contributions and that one can determine an associated uncertainty, which is not possible in many other methods. The main difficulty resides in defining a well-adapted power counting scheme.

For nuclear matter, the nuclear Fermi momentum $k_{F}$ enters as an additional scale. It is considered as small, of the same order as the pion mass. At saturation density it is given as $k_{F} \approx 263 \mathrm{MeV}$, which is indeed smaller than a typical hadronic scale. Based on this assumption, different power counting schemes have been developed. On the one hand, in the works of Kaiser, Fritsch, and Weise (2002, 2003, 2005), the vacuum chiral power counting has been applied directly to nuclear matter. On the other hand, Meißner, Oller, and Wirzba (2002), Oller, Lacour, and Meißner (2010), and Lacour, Oller, and Meißner (2011) argue that a propagating nucleon in the medium cannot always be counted in the standard way as $1 /|\vec{k}|$ with $|\vec{k}|$ being a typical nucleon three-momentum, but that there are "nonstandard" situations where it is to be counted as an inverse nucleon kinetic energy. In practice, within this nonstandard counting, certain classes of two-nucleon diagrams have to be resummed. Another difference is that these works include local nucleon-nucleon interactions fixed by free nucleon-nucleon scattering in addition to nucleon interactions mediated by pion exchange (Oller, Lacour, and Meißner, 2010; Lacour, Oller, and Meißner, 2011).

\section{f. Lattice methods}

The $a b$ initio approach to solve QCD numerically becomes extremely complicated at finite densities. The fermion determinant in the medium turns complex valued due to the appearance of the chemical potential. Consequently the integrals, which are evaluated in lattice QCD with Monte Carlo methods, have no longer positive weights. Different approaches to avoid this fermion sign problem have been suggested, e.g., reweighting techniques (Fodor and Katz, 2002), the introduction of complex chemical potentials (de Forcrand and Philipsen, 2002), or Taylor expansion schemes (Allton et al., 2002). A crucial parameter in all of these approaches is $\mu_{B} / T$, which for these analyses is typically of magnitude 1 or less. However, this value is significantly larger in the applications we are interested in, in particular, for NSs. Accordingly, no consistent cold matter or supernova EoS has been provided by lattice QCD so far.

In recent years lattice methods have been applied directly to nuclear systems; see Lee (2009) for a review. In the so-called "nuclear lattice effective field theory" (NLEFT), nucleons are treated as pointlike particles residing on the lattice sites. For the interactions, EFT nuclear forces are employed consisting of nucleon contact terms and potentially pion exchanges. 
These are represented on the lattice as insertions on the nucleon world lines. Because of the approximate $S U(4)$ spinisospin symmetry of nuclear forces, NLEFT suffers much less from the sign problem than lattice QCD. These methods have been applied successfully to light and medium mass nuclei (Epelbaum et al., 2014; Lähde et al., 2014), and to dilute neutron matter up to roughly one-tenth of nuclear matter saturation density (Lee, 2009). For the moment, however, there are no computations of denser systems. Lattice methods are used in the context of quantum Monte Carlo simulations, too (Wlazłowski et al., 2014).

\section{g. Perturbative $Q C D$}

QCD is asymptotically free, viz., the coupling constant decreases logarithmically with the energy (Gross and Wilczek, 1973; Politzer, 1974) and therefore, it is addressable by perturbative methods if the coupling constant turns small enough. This has been exploited to describe the thermodynamics of dense deconfined quark matter at finite temperature (Freedman and McLerran, 1977a, 1977b, 1977c). Recent efforts aimed to account for all second-order effects in an expansion of the thermodynamic pressure of deconfined QCD (Kurkela, Romatschke, and Vuorinen, 2010). This procedure gives valuable insights into the high-density limit of QCD and therefore provides an important constraint for the asymptotic behavior of the EoS at baryonic chemical potentials of several $\mathrm{GeV}$ (Kurkela et al., 2014). However, results from matching the EoS obtained within this approach directly to a nuclear matter EoS as suggested by Kurkela, Romatschke, and Vuorinen (2010) do not provide a conclusive answer as in this domain nonperturbative features cannot be neglected.

\section{h. Dyson-Schwinger approach}

The Dyson-Schwinger (DS) formalism is a nonperturbative approach to analyze QCD. It starts from a generating functional (the partition function of QCD). From there, coupled integral equations, the DS equations, are derived for the $n$ point Schwinger functions of the theory. Formally, this approach is similar to that of self-consistent Green's functions we discussed in Sec. III.B.1.a, but now based on the QCD Lagrangian. A further successful proving ground for this approach is QED. As in any many-body theory, every QCD Schwinger function couples to further Schwinger functions of higher order. This implies an infinite hierarchy of DS equations which can be solved practically only by introducing truncation schemes. There is no strict prescription how to truncate without erasing inherent properties (e.g., symmetries) of the original theory. The truncation scheme defines a specific model which then can be compared to experimental data and subsequently used to predict observables. The theoretical framework for vacuum and in-medium studies and numerous applications have been reviewed in detail in Roberts and Schmidt (2000), Alkofer and von Smekal (2001), and Roberts (2012). Despite the number of successful vacuum studies at zero and finite temperature it has been used only rather recently to compute EoSs of dense homogeneous quark matter in the deconfined phase (Chen et al., 2008, 2011, 2015; Klähn et al., 2010). Prominent topics are superconducting phases; see, e.g., Nickel, Wambach, and Alkofer (2006) and Alford et al. (2008), and the role of strange quarks (Nickel, Alkofer, and Wambach, 2006; Müller, Buballa, and Wambach, 2013). Further DS studies investigate the critical line in the QCD phase diagram; see, e.g., Fischer, Luecker, and Mueller (2011), Qin et al. (2011), Bashir et al. (2012), and Gutierrez et al. (2014). Although the DS approach promises insights from a QCD-based framework, no EoS has been obtained to date that covers the whole parameter space required to perform CCSN simulations. However, Klähn and Fischer (2015) showed that both the Nambu-JonaLasinio (NJL) model and the thermodynamic bag model (see Sec. III.B.2.b) can be understood as solutions of inmedium DS equations in rainbow approximation assuming a contact interaction for the gluon propagator.

\section{Phenomenological approaches}

Phenomenological approaches to describe dense matter are characterized by the use of effective interparticle interactions instead of realistic forces. They usually have a rather simple functional form in order to allow them to be used in several applications, i.e., not only uniform matter but in many cases also finite nuclei. However, their structure can be guided by symmetry principles, power counting arguments or insights from $a b$ initio approaches. Effective Hamiltonians can be derived from more fundamental forces using the formalism of density-matrix expansions (Negele and Vautherin, 1972; Dobaczewski, Carlsson, and Kortelainen, 2010; Stoitsov et al., 2010). In general, the actual parameters of the interactions are not directly calculated from underlying fundamental theories but they are determined empirically by fitting to (pseudo-) observables that are calculated in certain approximations of many-body theory. The interaction and the considered model space are not independent. Systematic extensions of the empirical approaches are not straightforward and usually require a refit of the model parameters.

Most empirical descriptions of dense matter rely on the MF approximation or use the closely related language of energy density functionals. Originally, the mean-field approximation corresponded to the Hartree approximation of the many-body state, i.e., a simple product of single-particle wave functions, but the term is often used to denote the HF approximation, too. The constituents are considered as quasiparticles with modified properties, e.g., effective masses that are different from their rest masses in the vacuum. Quantities such as the energy density or the pressure of the system can be expressed as functionals of the single-particle densities and an EDF is derived. It can be used as a starting point for the development of more refined EDFs that take into account features such as exchange and correlation effects going beyond the mean-field approximation. It is well known from the basic theorems in density functional theory (Hohenberg and Kohn, 1964; Kohn and Sham, 1965; Dreizler and Gross, 1990; Kohn, 1999; Fiolhais, Nogueira, and Marques, 2003) that an EDF exists which yields the exact energy of the system's ground state but its explicit form is not known. With suitable extensions of mean-field EDFs guided by empirical information one can try to come close to the exact EDF of the system, even if the interaction itself is not completely known. 
A further set of phenomenological EoSs can be characterized as purely parametric models which by themselves are not based on a description of the interaction of particles. Instead, a parametrized functional, typically of the energy density, is either assumed or fitted to microscopically motivated EoSs. These models are useful to analyze astrophysical data. Examples are single and piecewise polytrope fits for nuclear matter (Read et al., 2009) and a linear fit for quark matter (Zdunik, 2000; Alford, Han, and Prakash, 2013).

\section{a. Hadronic matter}

Self-consistent MF models are well developed. They are successful in describing the properties of systems composed of nucleons and were first used for bulk nuclear matter. Nowadays they are mainly applied in the description of finite nuclei; see, e.g., the review by Bender, Heenen, and Reinhard (2003). The approaches can be divided into two main classes: nonrelativistic and relativistic models. The main distinctions between them are the specific form of the interaction and the resulting dispersion relation of the quasiparticles.

Nonrelativistic approaches generally start from a Hamiltonian

$$
\hat{H}=\hat{T}+\hat{V}
$$

for the many-body system that contains the usual kinetic contribution

$$
\hat{T}=\sum_{i} \frac{\hat{p}_{i}^{2}}{2 m_{i}}
$$

and a potential term $\hat{V}$ that varies from model to model. Usually it is given as a sum

$$
\hat{V}=\sum_{i<j} \hat{V}_{i j}+\sum_{i<j<k} \hat{V}_{i j k}
$$

of two-body $\left(\hat{V}_{i j}\right)$ and three-body $\left(\hat{V}_{i j k}\right)$ interactions. The latter are required in order to reproduce the empirical saturation properties of nuclear matter. The energy of the system is calculated under certain assumptions for the form of the many-body wave function, usually within the HF approximation. Pairing effects can be considered in the Hartree-FockBogoliubov approximation. However, the original model interaction $\hat{V}$ cannot always be used in the pairing channel and a suitable pairing interaction has to be specified separately. Nonrelativistic approaches are in danger failing in the description of dense matter at high densities, e.g., the EoS can become superluminal.

Relativistic models are commonly formulated in a fieldtheoretical language by defining a Lagrangian density $\mathcal{L}$ that serves as the starting point in order to derive the field equations of the interacting particles. They constitute a set of coupled equations that have to be solved self-consistently. Expressions for the energy density and pressure are obtained from the energy-momentum tensor.

The foremost application of MF models is the description of finite atomic nuclei but nuclear matter properties are easily obtained once the parameters of the effective model interaction are determined. Depending on the selection of observables and preferences in the fitting of the effective interaction, different parametrizations are obtained. For the most common MF approaches, several hundred parameter sets are available in the literature. In the following, the most-used MF models, distinguished by the choice of the interaction, are considered.

- Mean-field models with Skyrme-type interactions: An effective zero-range interaction for HF calculations was introduced by Skyrme (1956, 1959). After the pioneering calculations of nuclei by Vautherin and Brink (1970, 1972), Brack and Quentin (1974b), and Beiner et al. (1975), it became very popular and found widespread use; see, e.g., the review article by Stone and Reinhard (2007). The basic form of the Skyrme interaction between nucleons 1 and 2 can be written as

$$
\begin{aligned}
\hat{V}_{12}^{(\text {Skyrme })}= & t_{0}\left(1+x_{0} \hat{P}_{\sigma}\right) \delta\left(\mathbf{r}_{12}\right) \\
& +\frac{1}{2}\left(1+x_{1} \hat{P}_{\sigma}\right)\left[\left(\hat{\mathbf{k}}^{\dagger}\right)^{2} \delta\left(\mathbf{r}_{12}\right)+\delta\left(\mathbf{r}_{12}\right) \hat{\mathbf{k}}^{2}\right] \\
& +t_{2}\left(1+x_{2} \hat{P}_{\sigma}\right) \hat{\mathbf{k}}^{\dagger} \cdot \delta\left(\mathbf{r}_{12}\right) \hat{\mathbf{k}} \\
& +\frac{1}{6} t_{3}\left(1+x_{3} \hat{P}_{\sigma}\right) \delta\left(\mathbf{r}_{12}\right) n_{B}^{\alpha}\left(\mathbf{R}_{12}\right) \\
& +i W_{0}\left(\hat{\sigma}_{1}+\hat{\sigma}_{2}\right) \cdot \hat{\mathbf{k}}^{\dagger} \delta\left(\mathbf{r}_{12}\right) \hat{\mathbf{k}}
\end{aligned}
$$

with parameters $t_{i}, x_{i}, \alpha$, and $W_{0}$. In Eq. (17) $P_{\sigma}=$ $\left(1+\hat{\sigma}_{1} \cdot \hat{\sigma}_{2}\right) / 2$ denotes the spin-exchange operator, $\hat{\mathbf{k}}=$ $\left(\nabla_{1}-\nabla_{2}\right) /(2 i)$ is the relative momentum, and $n_{B}$ is the total nucleon density. The relative coordinate and centerof-mass coordinate are defined by $\mathbf{r}_{12}=\mathbf{r}_{1}-\mathbf{r}_{2}$ and $\mathbf{R}_{12}=\left(\mathbf{r}_{1}+\mathbf{r}_{2}\right) / 2$, respectively. The contribution with factor $t_{3}$ is a generalization that originates from an explicit three-body term

$$
\hat{V}_{123}=t_{3} \delta\left(\mathbf{r}_{1}-\mathbf{r}_{2}\right) \delta\left(\mathbf{r}_{2}-\mathbf{r}_{3}\right)
$$

in the original Skyrme interaction and was converted to a density-dependent two-body interaction. The parameter $\alpha$ controls the strength of the repulsion. The original three-body interaction (18) corresponds to $\alpha=2$. The contribution with the factor $W_{0}$ generates the spin-orbit interaction in systems that are not spin saturated. For the description of nuclei, contributions from the Coulomb interaction have to be considered in addition. The potential (17) can be seen as an expansion in powers of the relative momentum $\mathbf{k}$. Since it stops at second order, the interaction cannot be applied reliably in cases where the momenta of the nucleons reach high values, e.g., in nuclear matter at densities substantially above saturation.

Evaluating the energy density from the Hamiltonian in $\mathrm{HF}$ approximation yields an EDF that depends on a number of single-particle densities and their spatial derivatives. Since the interaction is of zero range, exchange contributions are easily obtained and only local densities appear in the EDF. Besides the usual single-particle number densities $n_{i}$, the kinetic-energy densities $\tau_{i}$, the currents $\mathbf{j}_{i}$, the spin-orbit densities $\mathbf{J}_{i}$, the spin densities $\sigma_{i}$, etc. are relevant; see, e.g., Bender, 
Heenen, and Reinhard (2003) for details. In applications to nuclear matter, currents, spin densities, and spatial derivatives of all single-particle densities vanish. The energy density becomes a more-or-less simple functional in fractional powers of the number densities (Dutra et al., 2012). Obviously, an extrapolation to high densities can lead to divergences. Several extensions of the standard Skyrme functional have been proposed; see, e.g., Lesinski et al. (2007), Bender et al. (2009), Margueron et al. (2009, 2010, 2012), Margueron and Sagawa (2009), Chamel et al. (2011), Dutra et al. (2012), Hellemans, Heenen, and Bender (2012), and Davesne et al. (2015). We note that not every Skyrme-type EDF can be derived from simple two- and three-body potentials in a MF approximation. A number of well-calibrated parametrizations were proposed recently (Chabanat et al., 1997, 1998; Agrawal, Shlomo, and Au, 2005; Stone, 2005; Goriely, Chamel, and Pearson, 2009a, 2009b, 2013a, 2013b; Kortelainen et al., 2010, 2012, 2014; Washiyama et al., 2012). They predict many properties of nuclei close to experimental values with rather small deviations. The performance of 240 Skyrme parametrizations under nuclear matter constraints was studied by Rikovska Stone et al. (2003) and Dutra et al. (2012). Only a few satisfy all criteria that were selected. Some Skyrme forces show instabilities (Chamel and Goriely, 2010; Kortelainen and Lesinski, 2010; Hellemans et al., 2013; Navarro and Polls, 2013; Pastore et al., 2014) under particular conditions that may be cured with appropriate modifications of the EDF.

- Mean-field models with Gogny interaction: Instead of a zero-range force as in the Skyrme case, the use of finiterange interactions is an established approach in $\mathrm{MF}$ models. A sum of two Gaussians was suggested by Brink and Boeker (1967) for HF calculations. A review of phenomenological interactions in early HF models was given by Quentin and Flocard (1978). In order to obtain quantitatively reasonable results, a density-dependent effective two-body interaction was added by Gogny which leads to the present form

$$
\begin{aligned}
\hat{V}_{12}^{(\text {Gogny })}= & \sum_{j=1,2} \exp \left(-\frac{\mathbf{r}_{12}^{2}}{\mu_{j}^{2}}\right) \\
& \times\left(W_{j}+B_{j} \hat{P}_{\sigma}-H_{j} \hat{P}_{\tau}-M_{j} \hat{P}_{\sigma} \hat{P}_{\tau}\right) \\
& +t_{3}\left(1+x_{0} \hat{P}_{\sigma}\right) \delta\left(\mathbf{r}_{12}\right) n_{B}^{\alpha}\left(\mathbf{R}_{12}\right) \\
& +i W_{l s}\left(\hat{\sigma}_{1}+\hat{\sigma}_{2}\right) \cdot \hat{\mathbf{k}}^{\dagger} \delta\left(\mathbf{r}_{12}\right) \hat{\mathbf{k}}
\end{aligned}
$$

with parameters $\mu_{j}, W_{j}, B_{j}, H_{j}, M_{j}, t_{3}, \alpha$, and $W_{l s}$, and the isospin-exchange operator $P_{\tau}=\left(1+\hat{\tau}_{1} \cdot \hat{\tau}_{2}\right) / 2$ (Dechargé and Gogny, 1980). The density-dependent and the spin-orbit contributions have the form of the corresponding terms in the Skyrme interaction, although with a different notation of the parameters. Because of the finite-range part in the Gogny interaction, it is technically more involved to consider the exchange contributions to the energy density. On the other hand, divergences of a zero-range interaction are avoided in calculations involving pairing channels. Because of the more involved numerical calculations, there are only few parametrizations of the Gogny interaction that are used in practice (Goriely, Hilaire, and Koning, 2008). A collection of these parameter sets with a comparison to predictions of nuclear matter properties can be found in Sellahewa and Rios (2014).

- Relativistic mean-field and Hartree-Fock models: In relativistic approaches to nuclear matter and finite nuclei, a field-theoretical formalism is employed where nucleons are represented by Dirac four spinors $\psi_{i}$ and the nucleon-nucleon interaction is modeled by an exchange of mesons. This description, called quantum hadrodynamics (QHD), was originally seen as a fully fieldtheoretical approach (Fetter and Walecka, 1971; Chin and Walecka, 1974; Walecka, 1974; Serot and Walecka, 1986; Serot, 1992) and treated with the respective formalism. Later, the view of an effective description to be applied in rather simple approximations prevailed since nucleons as composite objects cannot be considered as fundamental degrees of freedom. The common starting point in QHD models is a Lagrangian

$$
\mathcal{L}=\mathcal{L}_{\text {nuc }}+\mathcal{L}_{\text {mes }}+\mathcal{L}_{\text {int }}
$$

that contains contributions of nucleons $i$

$$
\mathcal{L}_{\text {nuc }}=\sum_{i=n, p} \bar{\psi}_{i}\left(\gamma_{\mu} i \partial^{\mu}-m_{i}\right) \psi_{i}
$$

with rest mass $m_{i}$, of free mesons $\mathcal{L}_{\text {mes }}$, and an interaction term $\mathcal{L}_{\text {int }}$.

In early versions of the model only isoscalar mesons such as the (Lorentz-)scalar $\sigma$ meson and the (Lorentz-) vector $\omega$ meson were considered in order to model the long-range attraction and short-range repulsion of the nuclear interaction, respectively, in symmetric nuclear matter. Isovector mesons were added for the description of neutron-proton asymmetric systems. In most models, the vector isovector $\rho$ meson is considered, but an isospin-dependent splitting of the neutron and proton Dirac effective masses is obtained only when a scalar isovector $\delta$ meson is included. In contrast, the Landau effective masses are different in asymmetric matter even without a $\delta$ meson. Pseudoscalar mesons, such as the pion, or pseudovector mesons are relevant in models that treat exchange effects explicitly (Lalazissis et al., 2009). The mesons in the QHD approach share the same quantum numbers with their counterparts observed in experiments; however, they have to be seen as effective fields in the model that serve to capture the essential features of the strong interaction in the medium. With the standard choice of mesons, the contribution of free mesons to the Lagrangian (20) reads

$$
\begin{aligned}
\mathcal{L}_{\text {mes }}= & \frac{1}{2}\left(\partial_{\mu} \sigma \partial^{\mu} \sigma-m_{\sigma}^{2} \sigma^{2}\right) \\
& +\frac{1}{2}\left(\partial_{\mu} \vec{\delta} \cdot \partial^{\mu} \vec{\delta}-m_{\delta}^{2} \vec{\delta} \cdot \vec{\delta}\right) \\
& -\frac{1}{4} G_{\mu \nu} G^{\mu \nu}+\frac{1}{2} m_{\omega}^{2} \omega_{\mu} \omega^{\mu} \\
& -\frac{1}{4} \vec{H}_{\mu \nu} \cdot \vec{H}^{\mu \nu}+\frac{1}{2} m_{\rho}^{2} \vec{\rho}_{\mu} \cdot \vec{\rho}^{\mu}
\end{aligned}
$$


with the usual field tensors of the vector mesons $G_{\mu \nu}=$ $\partial_{\mu} \omega_{\nu}-\partial_{\nu} \omega_{\mu}$ and $\vec{H}_{\mu \nu}=\partial_{\mu} \vec{\rho}_{\nu}-\partial_{\nu} \vec{\rho}_{\mu}$. In most approaches, it is assumed that mesons couple minimally to nucleons leading to an interaction contribution of the form

$$
\begin{aligned}
\mathcal{L}_{\text {int }}= & -\sum_{i=n, p} \bar{\psi}_{i}\left[\gamma_{\mu}\left(g_{\omega} \omega^{\mu}+\vec{\tau} \cdot g_{\rho} \rho^{\mu}\right)\right. \\
& \left.+g_{\sigma} \sigma+g_{\delta} \vec{\tau} \cdot \vec{\delta}\right] \psi_{i},
\end{aligned}
$$

where $g_{i}(i=\omega, \sigma, \rho, \delta)$ denote the empirical coupling constants. Their values are obtained by fitting to properties of nuclear matter or finite nuclei. The coupling to scalar mesons modifies the Dirac effective mass $m_{i}^{*}$ of the nucleons. It is essential in order to obtain a realistic spin-orbit splitting in nuclei. From the Lagrangian (20) the field equations for nucleons and mesons are derived. They have to be solved self-consistently, usually in the RMF approximation, where meson fields are treated as classical fields and negative-energy states of the nucleons are neglected (no-sea approximation). Scalar and vector densities of nucleons appear as source terms for the mesons. Finally, a covariant energy density functional is obtained.

The basic version of QHD as discussed previously can qualitatively describe the feature of saturation in nuclear matter. It results from a competition of attractive scalar and repulsive vector self-energies $S_{i}$ and $V_{i}$ in the relativistic dispersion relation

$$
E_{i}=\sqrt{p^{2}+\left(m_{i}^{*}\right)^{2}}+V_{i}
$$

for a nucleon $i$ with momentum $p$ and Dirac effective mass $m_{i}^{*}=m_{i}-S_{i}$. With increasing density of the medium, the scalar potential $S_{i}$ rises more slowly than the vector potential $V_{i}$. The EoS becomes very stiff; nevertheless, the speed of sound does not exceed the speed of light. A quantitative description of nuclear matter and nuclei requires the extension of the simple Lagrangian density (20) in order to simulate a medium-dependent effective interaction. Several options have been explored in the literature.

In early extensions of the model, nonlinear (NL) selfinteractions of the mesons were considered by adding a contribution of the form

$$
\mathcal{L}_{\mathrm{nl}}=-\frac{A}{3} \sigma^{3}-\frac{B}{4} \sigma^{4}+\frac{C}{4}\left(\omega_{\mu} \omega^{\mu}\right)^{2}
$$

to Eq. (20). Cubic and quartic terms of the $\sigma$ meson were introduced by Boguta and Bodmer (1977) and satisfactory results were obtained; see, e.g., Reinhard et al. (1986) and Rufa et al. (1988). The addition of the quartic $\omega$ term by Sugahara and Toki (1994) was motivated by comparison of the scalar and vector potentials with DBHF. Later, selfcouplings of isoscalar and isovector mesons were used to modify the isospin dependence of the EoS (Müller and Serot, 1996; Furnstahl, Serot, and Tang, 1997; Todd-Rutel and Piekarewicz, 2005).

Instead of adding explicit new terms to the Lagrangian density (20), the coupling constants $g_{i}$ in Eq. (23) can be replaced by functionals $\Gamma_{i}$ of the nucleon fields. It is found that effective density-dependent (DD) nucleon-meson couplings can be extracted from the medium-dependent DBHF nucleon self-energies. Usually, a dependence of the couplings $\Gamma_{i}$ on the vector density ${ }^{5} n_{v}=\sqrt{j_{\mu} j^{\mu}}$ is assumed, which is defined in a covariant way with the nucleon current $j_{\mu}$. In the rest frame of a nucleus or nuclear matter, $n_{v}$ is identical to the baryon number density $n_{B}$. The density dependence of the couplings leads to so-called "rearrangement" contributions to the vector self-energies (Fuchs, Lenske, and Wolter, 1995). This is essential in order to obtain a thermodynamic consistent model. The functional form for the density dependence of the couplings with rational and exponential functions was suggested by a comparison to DBHF results in an early parametrization of the DD-RMF model that was fitted to binding energies of nuclei (Typel and Wolter, 1999). This approach was followed by several others (Nikšić et al., 2002, 2005; Long et al., 2004; Typel, 2005, 2010; Roca-Maza, Viñas et al., 2011). Alternative functions were considered by Gögelein et al. (2008). The density dependence of the meson-nucleon couplings was also directly derived from the nucleon self-energies (Hofmann, Keil, and Lenske, 2001a, 2001b; Gögelein et al., 2008), where the momentum dependence of the self-energies was mapped to an effective density dependence.

Details on applications of the NL and DD-RMF models can be found in Reinhard et al. (1986), Rufa et al. (1988), Reinhard (1989), Ring (1996), Serot and Walecka (1997), Bender, Heenen, and Reinhard (2003), Furnstahl (2004), Vretenar et al. (2005), and Nikšić, Vretenar, and Ring (2011). The models are employed without explicitly taking the antisymmetrization of the many-body wave function into account. The finite range of the effective interaction mediated by the meson exchange requires extra computational efforts in order to handle fully antisymmetrized many-body states similar to nonrelativistic Gogny HF calculations. In addition, an entirely new parameter set for the couplings has to be determined. Nevertheless, relativistic Hartree-Fock or Hartree-FockBogoliubov models were implemented (Long, Van Giai, and Meng, 2006; Meng et al., 2006; Long et al., 2007, 2010).

Treating the basic scalar-vector models as relativistic local quantum field theories, it was found that two-loop corrections lead to large contributions. It was concluded that the loop expansion does not converge and that the composite nature of the nucleons has to be respected by introducing nonlocal interactions as represented by form factors; see, e.g., Prakash, Ellis, and Kapusta (1992) and references therein.

Some generalizations of the RMF model extend the form of the nucleon-meson interaction from minimal couplings to couplings of the meson fields to derivatives of the nucleon fields. An early version is the model by Zimanyi and Moszkowski (1990), where scalar derivative couplings were introduced that could be transformed to particular

\footnotetext{
${ }^{5}$ Note that despite the name, the vector density is a Lorentz scalar.
} 
nonlinear couplings of nucleons to the $\sigma$ meson. General first-order derivative couplings were considered by Typel, von Chossy, and Wolter (2003) and Typel (2005). The approach was extended to derivatives of arbitrary high order by Gaitanos, Kaskulov, and Mosel (2009) and Gaitanos and Kaskulov $(2012,2013)$ in the nonlinear derivative (NLD) coupling model and studied in various versions (Chen, 2012, 2014; Antić and Typel, 2015; Gaitanos and Kaskulov, 2015). The main feature of the NLD approach is the dependence of the nucleon selfenergies not only on the density but also on the energy or momentum as in DBHF calculations. As a consequence, the momentum dependence of the nucleon optical potential in nuclear matter, which is extracted from the fitting of proton-nucleus scattering data in Dirac phenomenology (Hama et al., 1990; Cooper et al., 1993), can be reproduced up to nucleon energies of about $1 \mathrm{GeV}$.

The explicit appearance of meson fields in the Lagrangian density of RMF approaches is suppressed in socalled relativistic point-coupling (PC) models (Nikolaus, Hoch, and Madland, 1992; Rusnak and Furnstahl, 1997; Bürvenich et al., 2002; Zhao et al., 2010). Here fournucleon contact terms, including powers and derivatives thereof, appear with free prefactors that need to be determined. They can be seen as the result of expressing solutions of the meson field equations in the QHD approach as functions of the relativistic source densities and their spatial derivatives. This resembles the nonrelativistic Skyrme HF approach. A systematic expansion of $\mathcal{L}$ in various densities, currents, and their derivatives is possible by using power counting arguments from principles of effective field theory (Furnstahl, 2002). The form and parameters of the $\mathrm{PC}$ approach can also be constrained by in-medium $\chi$ EFT (Finelli et al., 2003, 2004, 2006).

Nuclear matter characteristics of 263 RMF parametrizations were compared recently by Dutra et al. (2014). Similar to the case of Skyrme HF models, only a very small number is consistent with all nuclear matter constraints considered in that publication.

- Quark-meson coupling model: An approach closely related to the previously discussed relativistic descriptions of matter and nuclei is the quark-meson coupling (QMC) model (Downum et al., 2006; Rikovska-Stone et al., 2007; Thomas et al., 2013; Whittenbury et al., 2014). It explicitly considers nucleons as bound states of quarks which couple to mesons in the surrounding medium. This leads to a polarization of the nucleon and the resulting mass shift is calculated selfconsistently. It can be expressed as a polynomial in the $\sigma$ meson field similar to NL-RMF models. Properties of matter are calculated in the HF approximation, including pions in addition to the standard scalar and vector mesons. With a small number of parameters, results of similar quality as in (non)relativistic meanfield models or EDFs are obtained.

- Other approaches to the nuclear energy density functional: Besides the models discussed previously that dominate the applications to the EoS, a number of independent alternative approaches were developed in the past. Instead of nonrelativistic effective interactions of the Skyrme or Gogny type, other forms were investigated, e.g., a density-dependent separable monopole interaction (Rikovska Stone et al., 2002) or three-range Yukawa (M3Y) type interactions (Nakada, 2003). The phenomenologically inspired approach of Fayans (1998), Fayans et al. (2000), and Fayans and Zawischa (2001) exploits the quasiparticle concept of Migdal's theory of finite Fermi systems. The Barcelona-CataniaParis (-Madrid) (BCP or BCPM) EDFs (Baldo et al., 2004, 2013; Baldo, Schuck, and Viñas, 2008) are constructed by interpolating between parametrizations of BHF results, obtained with realistic nucleon-nucleon potentials, for the EoS of symmetric nuclear matter and neutron matter. By adding appropriate surface and spinorbit contributions, an excellent description of finite nuclei is obtained with only a small number of parameters.

\section{b. Quark matter}

A proper QCD-based description of strongly interacting matter, in particular, in the vicinity of the predicted deconfinement phase transition, is desirable but currently not available since the theory is challenging to solve at finite chemical potentials. With a few exceptions, the prevailing approach for the hadron-quark transition region is to describe both phases separately and to interpolate in between in terms of a phase transition construction (see Sec. III.D). Therefore, QM in the following has to be understood as deconfined quark matter. In this phase one can think of quarks as actual particles or quasiparticles with no particularly complicated behavior or confinement properties. Then it is not surprising that typical approaches to describe quark matter show many similarities to RMF models for nuclear matter. To model quark matter correctly it is important though to understand and to account for the confinement mechanism in order to eventually understand the phase diagram of strongly interacting matter in the language of QCD. Therefore we briefly review corresponding developments as far as they concern the EoS of dense matter.

- Thermodynamic bag model: The simplest, but still widely applied model for QM is a limiting case of the MIT bag model (Chodos et al., 1974), which was originally developed to describe hadrons as quark bound states of finite size. Confinement in this model is accomplished by endowing the finite region with a constant energy per unit volume $B$. A special case is a highly excited hadron in which quarks would then behave as an ideal gas. The latter idea was followed to describe a system of homogeneous, deconfined quark matter (Farhi and Jaffe, 1984). The EoS is that of an ideal Fermi gas of three quark flavors, where the bag constant $B$ is added to the total energy density and subtracted from the pressure in order to maintain thermodynamic consistency. $B$ can be understood as the pressure difference of confined and deconfined quarks in vacuum. The value of $B$ can be determined from more sophisticated models (Cahill and Roberts, 1985). The bag constant arises not solely due to deconfinement but rather from the breaking of chiral symmetry. Consequently it is density dependent (Buballa and Oertel, 1999) as well as 
flavor dependent (Buballa, 2005). Already in perturbative QCD the thermodynamic bag models treatment of quarks as free noninteracting fermions does not hold and requires corrections (Freedman and McLerran, 1977c; Fraga, Pisarski, and Schaffner-Bielich, 2001), which can be generalized to a simple power series expansion of the pressure in the quark chemical potential (Alford et al., 2005). Similarly, a phenomenologically motivated expansion considers diquark contributions to the pressure (Alford and Reddy, 2003). Recently, an extension of the thermodynamic bag model was suggested which accounts for the breaking of chiral symmetry and the influence of vector interactions (Klähn and Fischer, 2015).

- Nambu-Jona-Lasinio-type models and extensions: One of the prominent features of QCD is the dynamical breaking of chiral symmetry as the mechanism that generates most of the hadron masses. The idea that the nucleon mass can be understood as the self-energy of a fermion in analogy to the energy gap of a superconductor has been developed at a time where the notion of quarks did not even exist (Nambu and Jona-Lasinio, 1961a, 1961b). The NJL model is based on a Lagrangian for a fermion field with a quartic, chirally symmetric local interaction of the form

$$
\mathcal{L}=\bar{\psi}\left(\gamma_{\mu} i \partial^{\mu}-m\right) \psi+G\left\{(\bar{\psi} \psi)^{2}+\left(\bar{\psi} i \gamma_{5} \vec{\tau} \psi\right)^{2}\right\}
$$

where $\psi$ is understood as a quark fermion spinor, $m$ is the bare quark mass, $G$ is a coupling constant, and $\vec{\tau}$ are the isospin matrices. The similarity to RMF models is evident. A Fierz transformation of the interaction gives access to all possible quark-antiquark interaction channels. These techniques have been explained in detail by Klevansky (1992) and Buballa (2005) for vacuum and inmedium applications. The coupling $G$ can be understood as a particular choice of a generalized form factor for nonlocal current-current interactions (Schmidt, Blaschke, and Kalinovsky, 1994; Bowler and Birse, 1995). An important feature of the approach is that the quartic terms - as a hallmark of NJL-type modelscan be shown to bosonize into baryon, meson, and diquark contributions to the partition function and hence to the thermodynamic potential (Kleinert, 1976; Roberts, Cahill, and Praschifka, 1988; Cahill, Praschifka, and Burden, 1989; Hatsuda and Kunihiro, 1994). This bosonization property is exploited to develop an understanding of hadrons as quark bound states in the medium (Bentz and Thomas, 2001; Wang, Wang, and Rischke, 2011; Blaschke et al., 2014). The original NJL model's success rests on its ability to describe the breaking of chiral symmetry. It fails to describe the infrared behavior of QCD which addresses confinement. It has therefore been suggested to extend the model by an imaginary chemical potential expressed in terms of the Polyakov loop as a possible order parameter of deconfinement (Fukushima, 2004; Ratti, Thaler, and Weise, 2006). A modification of the Polyakov loop potential in these PNJL models due to a finite quark chemical potential $\mu$ was introduced by Dexheimer and Schramm (2010). Further extensions result from variations of the previous models, e.g., nonlocal PNJL models without (Blaschke et al., 2008) and with (Contrera, Grunfeld, and Blaschke, 2014) $\mu$-dependent Polyakov loop potential. The PNJL model is used to study the QCD phase diagram at finite temperatures and densities (Fukushima, 2008). A further approach to account for confinement in NJL-type models suggests to introduce an infrared cutoff to remove unphysical quark-antiquark thresholds (Ebert, Feldmann, and Reinhardt, 1996). The similarity of the NJL model to the RMF approach for nuclear matter suggests that in analogy to the nonlinear Walecka model higher-order coupling channels, i.e., multiquark or quark-meson interactions, will affect, in particular, the high-density behavior of the EoS (Benic et al., 2015; Zacchi, Stiele, and Schaffner-Bielich, 2015).

\section{Clustered and nonuniform matter}

At subsaturation densities and not too high temperatures, nucleonic matter is no longer uniform since it becomes unstable with respect to variations in the particle densities. There are various criteria to identify the onset of instabilities, in both static and dynamic approaches; see Sec. III.D for details. Spatial structures can develop on different length scales. In stellar matter, nucleons can form nuclei or clusters of different sizes and shapes due to the interplay between the short-range nuclear interaction and the long-range electromagnetic interaction. If the size of the clusters is small as compared to their mean free path, the matter can still be described as a homogeneous system, however with cluster degrees of freedom in addition to nucleons and leptons. At higher densities, e.g., in the so-called pasta phases in the inner crust of NSs, the density variations have to be treated explicitly. In low-density cold matter, nuclei arrange themselves in a lattice and a crystal structure develops, e.g., in the outer crust of NSs, and a new length scale emerges. The appearance of cluster structures in matter can be treated in various approximations that differ mainly with respect to the choice of the basic degrees of freedom and to the description of interactions.

\section{Nuclear statistical equilibrium}

The most basic approach to describe clustered matter is given by NSE models, which are sometimes just called "statistical models." They are characterized by assuming a statistical ensemble of different nuclear species and nucleons in thermodynamic equilibrium. In particular, the chemical potentials of nuclei are not independent. They are given by Eq. (2). NSE models are not only used in simulations of CCSNe but also for nucleosynthesis calculations and in the context of thermonuclear supernovae; see, e.g., Seitenzahl et al. (2009).

In its simplest form, the ideal NSE, a mixture of noninteracting ideal gases assuming Maxwell-Boltzmann statistics is utilized. In chemistry, this description is known as "mass-action law." In the ideal NSE approach the abundance ratio of nuclei is determined by a Saha equation, which originally was used to describe the population of ionization 
states in atoms. Instead of classical Maxwell-Boltzmann statistics, the correct quantum statistics of the particles can also be implemented. Often the corresponding Fermi-Dirac distribution is adopted only for nucleons.

Standard NSE models do not take into account the effects of the strong interaction between the constituents explicitly, e.g., correlations in nucleon-nucleon scattering states are neglected. However, in some models the interactions in the nucleonic component are incorporated by employing a meanfield description of homogeneous matter; see Sec. III.B.2.a.

A large variety of NSE based models can be found in the literature, employing various extensions and different levels of sophistication. Here we summarize only the most important aspects which are typically discussed in the context of these models. For some selected models we give further details in Sec. V.

\section{a. Nuclear binding energies}

Realistic EoSs in the NSE model description require nuclear binding energies as basic input. Different approaches are used: On the one hand, values from theoretical models are employed. These can be simple mass formulas such as liquid-drop-like parametrizations or more detailed nuclear structure calculations; see, e.g., Myers and Swiatecki (1990, 1994), Möller et al. (1995), Lalazissis, Raman, and Ring (1999), Geng, Toki, and Meng (2005), and Koura et al. (2005). On the other hand, experimentally measured binding energies (Audi, Wapstra, and Thibault, 2003; Wang et al., 2012) are used directly. However, an extension of these mass tables from experiments is required with the help of theoretical approaches since exotic nuclei that are not yet studied experimentally can be encountered. Shell effects in the structure of nuclei have a significant impact on the distribution at low temperatures as illustrated in Sec. V.C. Binding energies of nuclei inside matter are modified as compared with their vacuum values. These medium effects are often introduced in NSE models in phenomenological approximations (see Sec. III.C.1.d).

\section{b. Excited states}

It is straightforward to include excited states of nuclei in an explicit way if their excitation energies are known experimentally. However, especially at high excitation energies and for very heavy or exotic nuclei, the experimental information on the levels and their properties is not complete. In this case, theoretical level densities or internal partition functions can be used (Fái and Randrup, 1982; Engelbrecht and Engelbrecht, 1991; Iljinov et al., 1992; Blinnikov et al., 2011). Alternatively, a temperature dependence of the binding energies is introduced (Botvina and Mishustin, 2010). The works of Rauscher and Thielemann (2000, 2001), and Rauscher (2003) provide nuclear partition functions in tabular form for temperatures up to $24 \mathrm{MeV}$ and a wide range of nuclear masses. These calculations are based on both experimental data and a backshifted Fermi-gas model. More recently, similar tables were provided by Goriely, Hilaire, and Koning (2008). Problems with divergences of the original Fermi-gas model (Bethe, 1936) at low excitation energy can be solved (Grossjean and Feldmeier, 1985). However, the general reliability of the employed densities of state formulas can be questioned. For an investigation of effects of excited states on the supernova EoS and the stellar collapse, see, e.g., Mazurek, Lattimer, and Brown (1979), Nadyozhin and Yudin (2004), and Liu, Zhang, and Luo (2007).

\section{c. Coulomb interaction}

In matter with the condition of electric charge neutrality, the Coulomb interaction among nucleons and nuclei is screened due to the background of electrons and possibly muons. Some NSE models neglect this Coulomb screening. Others include it by using only the one-body Wigner-Seitz approximation (Lattimer et al., 1985). However, there are very detailed calculations available that were obtained from the study of single-component and even multicomponent plasmas at different temperatures; see, e.g., Chabrier and Potekhin (1998), Chugunov and DeWitt (2009), Potekhin et al. (2009, 2013), and Potekhin, Chabrier, and Rogers (2009) and Sec. III.D.2 for more details. Typically the results are provided in the form of fitting formulas. The simulations have reached a high numerical precision and deviations from the linear mixing rule for binary plasmas were found to be small (DeWitt, Slattery, and Chabrier, 1996). For a discussion of different approximations of Coulomb interactions in supernova EoS and the application of some of the aforementioned models, see, e.g., Nadyozhin and Yudin (2005) and Blinnikov et al. (2011).

\section{d. Medium modifications of heavy nuclei}

Some statistical models employ explicit medium corrections of the binding energies of heavy nuclei. These can be due to temperature or due to the presence of unbound nucleons. In both cases, the surface and bulk properties of nuclei are modified as compared to the vacuum at zero temperature. One aspect is a temperature dependence of the symmetry energy (Dean, Langanke, and Sampaio, 2002; Agrawal et al., 2014) and of effective nucleon masses (Donati et al., 1994; Fantina et al., 2012). Obviously, such temperature effects are related to excited states and internal partition functions but the problem is approached from a different perspective. Effects of the unbound nucleons on nuclei are often extracted from nucleons-in-cell calculations (see Sec. III.C.6). For instance, Papakonstantinou et al. (2013) and Aymard, Gulminelli, and Margueron (2014) calculated the binding energy shifts for Skyrme interactions in the local-density approximation and in the extended Thomas-Fermi (TF) approximation, respectively. It was pointed out that the definition of the binding energy shifts has to be consistent with the definition of clusters where one has to distinguish coordinate-space and energy-space clusters.

\section{e. Cluster dissolution}

The application of the standard NSE is limited to rather low densities. This model cannot describe the dissolution of nuclei with increasing densities, the Mott effect, which is mainly driven by the Pauli principle (Röpke, Münchow, and Schulz, 1982; Röpke et al., 1983). When the nuclear saturation density is approached, a transition to uniform nucleonic matter is often enforced with the help of the excluded-volume mechanism, which represents a classical, phenomenological approach to describe the dissolution of nuclei at high densities in a 
geometrical picture. Different variants of excluded-volume effects can be found in the literature. In the simplest case, the total volume of the thermodynamic system is replaced by the so-called free volume that is the total volume reduced by the volume occupied by the particles of finite size (Rischke et al., 1991). This approach is well known from the EoS of a van der Waals gas. General expressions for excluded-volume effects can be found in Yudin (2010, 2011) and Typel (2016). More detailed models solve for the exact canonical partition function, taking into account finite volumes of the particles and/or assuming a certain geometry of the particles. An important example is the hard-sphere model (Carnahan and Starling, 1969; Mulero, 2008). Note that the excluded-volume mechanism is also commonly used in the context of relativistic heavy-ion collisions (HICs) to describe the freeze-out of particles and their yields in a hadron resonance gas model (Gorenstein, Petrov, and Zinovjev, 1981; Andronic et al., 2012). A problem of the excluded-volume approach is the occurrence of a superluminal speed of sound at high densities and hence the EoS becomes acausal (Rischke et al., 1991; Venugopalan and Prakash, 1992).

\section{Single nucleus approximation}

Instead of considering the distribution of all nuclei, the chemical composition is sometimes simplified by assuming a representative single heavy nucleus, unbound nucleons, and possibly $\alpha$ particles and other light nuclei in the description. Burrows and Lattimer (1984) showed that this so-called single nucleus approximation (SNA) has only a small impact on thermodynamic quantities. However, there can be significant differences between the average mass and charge number of heavy nuclei in a full NSE model and the corresponding values of the representative nucleus employing the SNA (Souza et al., 2009) (see also Sec. V.C). Furthermore, the conclusions of Burrows and Lattimer (1984) are not applicable if the composition is dominated by light nuclei. In this case it is not possible to consider a "representative light nucleus" due to the small number of nuclei involved and the large variability of their binding energies (Hempel et al., 2012, 2015). We point out that considering a statistical ensemble of all nuclei, i.e., going beyond the SNA, is particularly relevant for the determination of electron-capture rates during core collapse (see Sec. VI.B.1).

\section{Virial expansion}

Correlations between the constituents of a low-density gas of particles at finite temperature can be considered in the virial equation of state (VEoS). It provides corrections to the NSE approach to clustered matter. The original formulation goes back to Beth and Uhlenbeck $(1936,1937)$ and uses a description based on the grand canonical ensemble. The $\mathrm{VEoS}$ relies on a series expansion of the grand canonical potential

$\Omega\left(T, V,\left\{\mu_{i}\right\}\right)=-T V\left(\sum_{i} \frac{b_{i}}{\lambda_{i}^{3}} z_{i}+\sum_{i j} \frac{b_{i j}}{\lambda_{i}^{3 / 2} \lambda_{j}^{3 / 2}} z_{i} z_{j}+\cdots\right)$ in powers of the particle fugacities $z_{i}=\exp \left[\left(\mu_{i}-m_{i}\right) / T\right]$, where $\mu_{i}$ is the chemical potential of particle species $i$ including the rest mass $m_{i}$. The quantities $\lambda_{i}=\sqrt{2 \pi / m_{i} T}$ denote the thermal wavelengths and $b_{i}$ are the degeneracy factors of the single particles. The virial coefficients $b_{i j}, b_{i j k}$, etc. are simple functions of the temperature. They contain information on the two-, three-, etc., many-body correlations in the system. In particular, the second virial coefficients

$$
b_{i j}=\frac{\lambda_{i}^{3 / 2} \lambda_{j}^{3 / 2}}{2 \lambda_{i j}^{3}} \int d E \exp \left(-\frac{E}{T}\right) D_{i j}(E)
$$

with $\lambda_{i j}=\sqrt{2 \pi /\left(m_{i}+m_{j}\right) T}$ depend only on the energies $E_{l, k}^{(i j)}$ of the two-particle bound states and the two-body scattering phase shifts $\delta_{l}^{(i j)}$ in channels $l$ that appear in the function

$$
D_{i j}(E)=\sum_{l}\left[\sum_{k} g_{l, k}^{(i j)} \delta\left(E-E_{l, k}^{(i j)}\right)+\frac{g_{l}^{(i j)}}{\pi} \frac{d \delta_{l}^{(i j)}}{d E}\right]
$$

with appropriate degeneracy factors $g_{l, k}^{(i j)}$ and $g_{l}^{(i j)}$. Note that the separation of bound state (first term) and scattering contributions (second term) is not unique, since a partial integration of the energy integral (28) leads to equivalent expressions with a different partitioning. Because experimental information can be used directly in the evaluation, a modelindependent approach is obtained. Quantum statistical (QS) effects can be incorporated easily. For contributions beyond second order see, e.g., Pais and Uhlenbeck (1959), Dashen, Ma, and Bernstein (1969), Bedaque and Rupak (2003), and $\mathrm{Liu}, \mathrm{Hu}$, and Drummond (2009). The VEoS is only applicable for small fugacities $z_{i} \ll 1$ or equivalently $n_{i} \lambda_{i}^{3} \ll 1$ with the particle number density $n_{i}$. These thermodynamic conditions are found, e.g., in the neutrino sphere of supernovae. In present applications of the VEoS to stellar matter only correlations on the level of the second virial coefficient due to the strong interaction are considered.

\section{Quantum statistical approach}

As mentioned, the transition from inhomogeneous to uniform matter cannot be easily described within the NSE approach nor within the VEoS. For that purpose, NSE models are extended phenomenologically by adding a classical excluded-volume correction (see Sec. III.C.1.e). A systematic description of correlations and, in particular, the Mott effect in an interacting many-body system is given by the QS approach (Röpke, Münchow, and Schulz, 1982; Röpke et al., 1983).

In general, many-body methods can provide spectral functions that contain all information on correlations. Prominent peaks in a spectral function can be identified with the corresponding quasiparticles, e.g., deuterons in nucleonic matter. As an approximation, quasiparticles with shifted energies can be introduced in the practical calculation of an EoS. These energies depend on the nucleon densities, the temperature, and the momentum of the quasiparticle in the medium. They include effects of Pauli blocking and can be parametrized with more or less sophistication (Röpke, 2009, 
2011; Typel et al., 2010). The energy shifts enter in the determination of the particle densities and finally in the EoS.

Going beyond the simple quasiparticle approximation for the spectral function in the QS approach leads to a form of the grand canonical potential that closely resembles the corresponding expression of the VEoS. This generalized BethUhlenbeck approach was formulated and studied in detail with realistic two-nucleon potentials (Schmidt, Röpke, and Schulz, 1990; Stein, Morawetz, and Röpke, 1997). In this case, the EoS can be formulated with a modified expression for the second virial coefficient. The quasiparticle energies in the bound state contribution are affected by the in-medium energy shifts and the phase shifts in the scattering contribution have to be calculated from the in-medium $T$ matrix for twobody scattering.

\section{Generalized relativistic density functional}

The modification of cluster binding energies in the medium, which is a basic feature of the QS approach, can be implemented in quasiparticle models. The generalized relativistic density functional (gRDF) approach (Typel et al., 2010, 2014; Typel, 2013) is such a model. It is an extension of a RMF model with density-dependent nucleon-meson couplings that is constrained by fits to properties of finite nuclei. In the gRDF model, nucleons and clusters are included as explicit degrees of freedom. Two-nucleon scattering correlations are described by effective resonances in the continuum with parameters that are adjusted in order to reproduce the model-independent virial EoS at low densities (Voskresenskaya and Typel, 2012). All particles couple to the meson fields with appropriately scaled strengths resulting in density-dependent scalar and vector self-energies. In addition, mass shifts of composite particles are implemented in a parametrized form, which are derived from the QS approach, in order to account for Pauli blocking from the nucleons in the medium. A further change of the cluster binding energies is caused by the screening of the Coulomb field due to the electronic environment in stellar matter. The density dependence of the binding energy shifts and meson-nucleon couplings leads to rearrangement contributions in the vector self-energy and thermodynamic quantities, which guarantee a thermodynamically consistent approach. The model interpolates between the correct low-density limit given by the virial EoS and the suprasaturation case of purely nucleonic matter. Medium-dependent mass shifts of light clusters were also incorporated in other models that study pasta phases in compact star matter (Avancini et al., 2012; Pais, Chiacchiera, and Providência, 2015).

\section{Nucleons-in-cell calculations}

In all models that were previously described, matter is treated as a uniform system of interacting particles. They are assumed to be pointlike or to have a finite volume. The formation of inhomogeneous structures in subsaturation matter at low temperatures can be studied in calculations where a nonuniform distribution of nucleons inside a cell of given shape and size is considered. Here quantal and classical methods can be distinguished. In stellar matter, electrons are usually not treated explicitly but considered as a uniform background gas. The methods are mostly applied to study the formation of clusters or pasta phases in the crust of neutron stars, the corresponding phase transitions, and the effects of the Coulomb interaction (see Sec. III.D). Beyond static properties for an EoS, the dynamical response, e.g., in neutrino scattering, or hydrodynamic quantities such as viscosities and conductivities can also be studied.

In the classical molecular dynamics (CMD) approach (Dorso, Molinelli, and Lopez, 2011; Horowitz et al., 2011; Dorso, Giménez Molinelli, Nichols, and Lopez, 2012; Piekarewicz and Toledo Sanchez, 2012; Schneider et al., 2013; Giménez Molinelli et al., 2014; Giménez Molinelli and Dorso, 2015), a certain number of nucleons is placed inside a box of given volume to reproduce a fixed density. The velocities of the particles are chosen to represent a Maxwellian distribution at given temperature. The particles interact via a two-body potential where the Coulomb contribution is essential. The time evolution of the system is followed by solving a set of classical coupled equations of motion, which is possible even for a rather large number of particles. In quantum molecular dynamics (QMD) models (Maruyama et al., 1998; Kido et al., 2000; Watanabe et al., 2002a, 2002b, 2003a, 2004, 2009; Matsuzaki, 2006; Watanabe, 2007; Sonoda et al., 2008; Maruyama, Watanabe, and Chiba, 2012), particles are not treated as pointlike objects as in CMD calculations but as wave packets of Gaussian shape. The motion of the centroid of the wave package is classically followed. Quantum statistical effects, such as antisymmetrization or shell effects, are not accounted for in molecular dynamics (MD) simulations. The Pauli exclusion principle can be incorporated approximately with appropriately designed contributions to the potentials. Central questions of MD calculations are the fragment recognition (Dorso and Randrup, 1993; Strachan and Dorso, 1997), the chemical composition (Horowitz, Berry, and Brown, 2007; Horowitz and Berry, 2009; Dorso, Giménez Molinelli, López, and Ramirez-Homs, 2012; Caplan et al., 2014), the appearance of different, sometimes complicated, shapes in the density distribution and their topological characterization (Watanabe et al., 2003b; Dorso, Giménez Molinelli, and López, 2012; Alcain, Giménez Molinelli, Nichols, and Dorso, 2014; Schneider et al., 2014; Horowitz et al., 2015), and phase transitions (Watanabe et al., 2005; Alcain, Giménez Molinelli, and Dorso, 2014). Structure functions and quantities related to the dynamical response can be extracted as well (Horowitz et al., 2004, 2005; Horowitz, Perez-Garcia, and Piekarewicz, 2004; Caballero et al., 2008; Horowitz and Berry, 2008, 2009).

A description of matter inside a cell based on particle density distributions instead of localized classical particles is a widely adopted approach for inhomogeneous systems. A number of different methods is available. They differ in the physical input, the approximations, and the numerical complexity. Many approaches that model the formation of large nuclei surrounded by a gas of nucleons employ the WignerSeitz approximation where the size of the cell is determined by the neutrality condition, i.e., the total charge of baryonic matter inside the volume considered is compensated by the electronic charge. At low densities, a spherical cell, which is centered around individual nuclei, is usually assumed. When 
other geometries, mostly rectangular shapes, are considered, it is common to presume a periodic density distribution in space.

The (compressible) liquid-drop model (LDM) belongs to the class of microscopic-macroscopic approaches where the energy of matter inside the cell is parametrized with several individual contributions such as bulk, surface, etc. (Baym, Bethe, and Pethick, 1971; Lattimer, 1981; Lamb et al., 1983; Ravenhall, Pethick, and Wilson, 1983; Lattimer et al., 1985; Lattimer and Swesty, 1991; Lorenz, Ravenhall, and Pethick, 1993; Watanabe, Iida, and Sato, 2000; Douchin and Haensel, 2001; Oyamatsu and Iida, 2007; Nakazato, Oyamatsu, and Yamada, 2009; Furusawa et al., 2011; Nakazato, Iida, and Oyamatsu, 2011; Furusawa, Sumiyoshi et al., 2013). They depend on the size of the heavy nucleus and the density of the surrounding gas of nucleons. The detailed structure of the expressions can be guided by energy density functionals, often nonrelativistic Skyrme parametrizations. Because of its convenience in the numerical application, the LDM is one of the earliest approaches to model inhomogeneous stellar matter.

Other widely employed methods are the TF approximation or extensions thereof. Here the density distribution of fermions in the cell is determined using a given EDF for nucleonic matter. In the most simple form, a local-density approximation of nuclear matter is obtained, but corrections related to surface effects and the finite range of interactions, can be incorporated in the calculation. The shape of the density distributions can be given by a simple functional form with few parameters or can be determined fully self-consistently. Technically, the free energy of the cell is minimized variationally and the chemical potentials of the nucleons are obtained for fixed particle numbers. Both nonrelativistic and relativistic (Sumiyoshi, Oyamatsu, and Toki, 1995; Cheng, Yao, and Dai, 1997; Shen et al., 1998a, 2011; Avancini et al., 2008, 2009; Avancini, Barros et al., 2010; Avancini, Chiacchiera et al., 2010; Okamoto et al., 2012; Zhang and Shen, 2014) models are available. The main disadvantage of the TF approach is that shell effects are not included. They can be incorporated with the Strutinski method (Brack, Jennings, and Chu, 1976; Brack, Guet, and Hakansson, 1985; Onsi et al., 2008; Pearson et al., 2012, 2015) but this correction to the TF approach is not widely used for astrophysical EoS. The formation of inhomogeneous matter inside cells of various shapes is also studied by using the density functional theory (DFT) formalism in relativistic approaches (Maruyama et al., 2005; Gögelein and Müther, 2007), where shell effects are included but only in the mean-field approximation without explicit particle exchange.

The next level of complexity is reached in HF calculations, which take the antisymmetrization of the nuclear manybody wave function within the cell fully into account. This approach is most frequently utilized with nonrelativistic potentials such as the zero-range Skyrme interaction, e.g., in the pioneering works of Ravenhall, Bennett, and Pethick (1972), Negele and Vautherin (1973), Bonche and Vautherin (1981, 1982), and Bonche, Levit, and Vautherin (1985). Shell effects were found to disappear quickly with increasing temperature at 2-3 MeV and nucleon pairing effects are relevant only below approximately $1 \mathrm{MeV}$ (Brack and
Quentin, 1974a, 1974b). The evolution of the density distribution inside the cells and consequences for the EoS are investigated in several works; see, e.g., Sil et al. (2002), Magierski, Bulgac, and Heenen (2003), Gögelein and Müther (2007), Gusakov, Kantor, and Haensel (2009a), Newton and Stone (2009), Pais and Stone (2012), Papakonstantinou et al. (2013), and Pais, Newton, and Stone (2014). Also timedependent HF calculations were employed, both in the standard formalism (Schuetrumpf et al., 2013a, 2013b, 2015) and in an extended approach using dynamical wavelets for single-particle wave functions (Sebille, Figerou, and de la Mota, 2009; Sebille, de la Mota, and Figerou, 2011). A correct treatment of antisymmetrization beyond the individual cell volume was developed recently (Vantournhout, Jachowicz, and Ryckebusch, 2011; Vantournhout et al., 2011; Vantournhout and Feldmeier, 2012).

\section{Phase transitions}

Theoretical models for the EoS are mostly concerned with calculations of single phases where a thermodynamic potential is extremized locally for its set of natural state variables (see Sec. II.A). In the case of thermodynamic instabilities, the equilibrium state of the system is obtained from a global minimization or maximization of the appropriate thermodynamic potential allowing for a phase transition with coexistence of different phases, i.e., macroscopic regions in space with different values of the various densities but identical intensive variables (Landau and Lifshitz, 1980).

Examples of phase transitions in the present context are the liquid-gas phase transition in nuclear matter (Barranco and Buchler, 1980; Müller and Serot, 1995; Gulminelli et al., 2003; Hempel et al., 2013), the hadron-quark transition, which is expected to occur at very large baryon number densities and/or temperatures (Collins and Perry, 1975; Prakash, Cooke, and Lattimer, 1995; Steiner, Prakash, and Lattimer, 2000; Mishustin et al., 2002; Bhattacharyya, Mishustin, and Greiner, 2010; Hempel et al., 2013; Yasutake et al., 2013), and the gas-liquid-solid phase transition, which is relevant in the formation of the crystalline crust during the cooling of PNSs (Chamel and Haensel, 2008). A phase transition could also be caused by the appearance of new particle species such as hyperons in dense hadronic matter (Schaffner-Bielich and Gal, 2000; Schaffner-Bielich et al., 2002; Gulminelli, Raduta, and Oertel, 2012; Gulminelli et al., 2013).

\section{Thermodynamic description of phase transitions}

In the following, $F\left(T,\left\{N_{i}\right\}, V\right)$ is chosen as the thermodynamic potential for the discussion. The free energy describes the equilibrium thermodynamics of a system if it is a convex function of the extensive variables, i.e., $\left\{N_{i}\right\}$ and $V$, and a concave function of the temperature $T$. Then the conjugate intensive variables, the chemical potentials $\mu_{i}=\partial F /\left.\partial N_{i}\right|_{T,\left\{N_{j \neq i}\right\}, V}$, and the pressure $p=-\partial F /\left.\partial V\right|_{T,\left\{N_{i}\right\}}$ are constant throughout the volume $V$. The free energy of a particular theoretical model is locally convex in the subspace of extensive variables if all eigenvalues of the stability matrix $M$ with entries 


$$
M_{i j}=\left.\frac{\partial^{2} F}{\partial Q_{i} \partial Q_{j}}\right|_{T, Q_{k, k \neq i, k \neq j}}
$$

are positive. Here $Q_{i}, Q_{j}$, and $Q_{k}$ are variables from the set of the conserved charges $\left\{N_{i}\right\}$ or the volume $V$. If at least one eigenvalue of $M$ is zero or negative, this point in the space of variables is metastable or unstable, respectively. All unstable points are enclosed by the so-called spinodal in the space of conserved charge numbers $N_{B}, N_{Q}$, etc. Besides this thermodynamic criterion there are other dynamical approaches to identify the instability region, e.g., collective excitations with random phase approximation calculations, the Vlasov equation formalism, or Fermi liquid theory (Pethick and Ravenhall, 1988; Heiselberg, Pethick, and Ravenhall, 1993; Margueron, Navarro, and Blottiau, 2004; Brito et al., 2006; Providência, Brito, Avancini et al., 2006; Providência, Brito, Santos et al., 2006; Ducoin, Margueron, and Chomaz, 2008; Ducoin et al., 2008).

The spinodal is enclosed by the binodal that connects all points with identical temperature, chemical potentials, and pressure. Points inside the binodal belong to the phase coexistence region. Here the free energy for given conserved total charge numbers can be lowered as compared to the locally calculated value by considering the coexistence of two phases $p=\mathrm{I}$, II with different volumes $V^{p}$ such that $V^{\mathrm{I}}+V^{\mathrm{II}}=V$. The particle numbers $N_{i}^{p}$ in the individual phases are in general different but all chemical potential are identical $\mu_{i}^{\mathrm{I}}=\mu_{i}^{\mathrm{II}}$ in the two phases as well as the other intensive variables $\left(P^{\mathrm{I}}=P^{\mathrm{II}}, T^{\mathrm{I}}=T^{\mathrm{II}}\right)$. This is called the Gibbs condition for thermodynamic equilibrium. Points in coexistence always lie on a binodal. Hence, it is sufficient to know the thermodynamic potential on this line or surface to construct the system properties for thermodynamic conditions inside the binodal.

If there is only a single conserved charge, it is easily checked whether the free energy of a particular theoretical model is a convex function of the only conserved charge for given $T$ and $V$. If not, the binodal degenerates to two separate points and the well-known Maxwell construction of phase transitions with isotherms in the pressure-density diagram is obtained. By changing the temperature of the system, the two distinct points in coexistence can collapse into a single point that defines the critical point and the corresponding critical temperature and critical pressure. If this topology applies, it is possible to move from one phase to the other around the critical point without crossing the binodal in the temperaturedensity plane. In some cases, e.g., for the quark-hadron phase transition, there is often no consistent model for the complete range of the thermodynamic variables and two different models are used. Then a transition from one phase to the other without crossing a phase separation line is not possible (Hempel et al., 2013).

In the case of more than one conserved charge the complete set of Gibbs conditions applies and the topology of the binodals and spinodals becomes more complex (Barranco and Buchler, 1980; Glendenning, 1992; Müller and Serot, 1995; Iosilevskiy, 2010; Hempel et al., 2013). New features appear: In the higher dimensional parameter space, the critical point turns into a critical line or critical hypersurface and several topological end points can be defined. However, it is always possible to map the Gibbs construction with several independent charge numbers to a technically more simple Maxwell construction (Ducoin, Chomaz, and Gulminelli, 2006; Typel et al., 2014). This is achieved by applying Legendre transformations to the free energy $F$ that replace all conserved charge numbers except one with the corresponding chemical potentials. Thereby a modified thermodynamic potential is found that depends only on a single-particle number as in the standard Maxwell case.

\section{Coulomb effects}

In models of the EoS for NSs and CCSNe the equilibrium with respect to the strong as well as the electromagnetic interaction between all constituents has to be considered. As a consequence, the phase structure of dense matter is substantially affected by the interplay of these short- and longrange forces in competition with entropy. In addition, the specific condition of charge neutrality applies. At not too high temperatures the appearance of clusters and crystalline structures is expected (see also Sec. III.C). Closely connected to both features is the possible occurrence of pasta phases.

If macroscopic phases coexist, different assumptions for the treatment of charge neutrality can be made. Either one requires local charge neutrality, i.e., each phase is charge neutral, or the system is charge neutral as a whole and the phases are allowed to carry a net charge. We note that the assumption of charged phases but global charge neutrality contradicts the assumption of the thermodynamic limit, if interpreted strictly, as the Coulomb energy would diverge for such a system. Nevertheless, it is considered as a reasonable simplification in many situations; see Martin and Urban (2015) for a discussion regarding the inner NS crust. Depending on the choice for realizing the charge neutrality condition, the chemical equilibrium conditions for phase coexistence (Hempel, Pagliara, and Schaffner-Bielich, 2009) lead to different qualitative properties of the phase transition (Glendenning, 1992; Iosilevskiy, 2010; Hempel et al., 2013), in particular, to the feature of Coulomb frustration (Gulminelli et al., 2003; Chomaz et al., 2007; Napolitani et al., 2007; Hasnaoui and Piekarewicz, 2013).

In more advanced approaches, surface effects and the finiterange of interactions are explicitly taken into account. A crucial ingredient is the surface tension between phases. Typically, for high surface tensions, the phases tend to approach a configuration that resembles the case of local charge neutrality (Heiselberg, Pethick, and Staubo, 1993; Maruyama et al., 2008; Yasutake et al., 2013). For very low surface tensions, however, the configuration can be similar to the case of global charge neutrality without finite-size effects.

The liquid-gas phase transition in nuclear matter predicts the coexistence of high-density and low-density phases of macroscopic size below a critical temperature. If Coulomb effects are included, electrons have to be added to compensate the positive proton charge. Phase transitions with macroscopic, charge-neutral phases in coexistence would create large electric fields at the interfaces that the system tries to avoid (Voskresensky, Yasuhira, and Tatsumi, 2003). This can 
be seen in the analysis of instabilities where density fluctuations of certain wavelengths are preferred. Clusters of finite size emerge that are surrounded by a low-density gas of nucleons. The density of the electrons throughout the system is nearly constant due to the large incompressibility of such a high-density Fermi liquid. Often a constant electron density is assumed, with some exceptions; see, e.g., Maruyama et al. (2005), Endo et al. (2006), and Yasutake, Maruyama, and Tatsumi (2011). The formation of clusters in uniform nuclear matter is well studied in several models that consider the distribution of nucleons and electrons in a cell of given geometry (see Sec. III.C.6). Usually, the Wigner-Seitz approximation is applied. At low temperatures and rather low densities, a single cluster in a spherical cell is the preferred geometry (Lamb et al., 1978; Douchin and Haensel, 2000). With increasing density it becomes more advantageous to develop structures of cylindrical or planar geometry and a sequence of pasta phases is found; see, e.g., Watanabe and Iida (2003) and Gupta and Arumugam (2013) and references given in Secs. III.C.6 and V.A.1. This was observed in early models for cells with different symmetry with simple energy density functionals (Hashimoto, Seki, and Yamada, 1984; Oyamatsu, Hashimoto, and Yamada, 1984; Williams and Koonin, 1985; Oyamatsu, 1993). In principle, every change from one geometry to another represents a phase transition of its own. More refined calculations with less restrictions to the spatial distributions of the densities found that transitions from clustered matter at low densities to uniform matter at high densities exhibit weaker discontinuities, i.e., the phase transitions are much less pronounced (Newton and Stone, 2009; Pais, Newton, and Stone, 2014). A nearly complete quenching of the traditional liquid-gas phase transition can occur (Gulminelli et al., 2003).

When stellar matter is cooled down at a given density, the size of clusters grows as observed, e.g., in spherical WignerSeitz cell calculations. At very low densities, the average distance between the clusters is large and the short-range strong interaction can practically be neglected. Coulomb and thermal energies drive the thermodynamic behavior of the system that can be seen as a plasma of ions and electrons. At very low temperatures a phase transition from the gas phase to a crystalline phase is expected (Salpeter, 1961; Baym, Pethick, and Sutherland, 1971). The ratio of the strengths of the Coulomb interaction and of the thermal energy is measured by the parameter

$$
\Gamma_{i}=\frac{Z_{i}^{5 / 3} e^{2}}{a_{e} T}
$$

with the charge $Z_{i}$ of the cluster and the electronic length scale

$$
a_{e}=\left(\frac{3 n_{e}}{4 \pi}\right)^{1 / 3}
$$

depending on the electron density $n_{e}$. When the temperature approaches zero, $\Gamma_{i}$ diverges. Fully ionized electron-ion plasmas have been studied in detail (Brush, Sahlin, and Teller, 1966; Hansen, 1973; Pollock and Hansen, 1973; Farouki and Hamaguchi, 1993; Chabrier and Potekhin, 1998; Potekhin and Chabrier, 2000, 2010; Chabrier,
Douchin, and Potekhin, 2002; Daligault, 2006; Cooper and Bildsten, 2008; Potekhin et al., 2009; Potekhin, Chabrier, and Rogers, 2009). From classical Monte Carlo simulations (see Sec. III.C.6) of a one-component plasma (OCP) it is known that the phase transition from the gas to the crystal phase occurs at $\Gamma_{i} \approx 175$. The exact value will depend on the details of the theoretical model. In particular, the often employed Wigner-Seitz approximation is insufficient to capture the correlations that are induced by the Coulomb interaction and determine the location of the phase transition. For a mixture of different ionic species, corrections to the OCP result apply (Ogata et al., 1993; Chabrier and Potekhin, 1998; Nadyozhin and Yudin, 2005; Chugunov and DeWitt, 2009). For $\Gamma_{i} \rightarrow 0$ the Debye screening limit in a plasma is obtained. For $\Gamma_{i} \rightarrow \infty$ a body-centered cubic (bcc) lattice of ions immersed in a uniform sea of electrons is found as the ground state (Baym, Bethe, and Pethick, 1971; Baym, Pethick, and Sutherland, 1971). At finite temperatures lattice vibrations contribute to the thermodynamic potential of the system (Baiko, Potekhin, and Yakovlev, 2001). With increasing temperature these thermal excitations will lead to the melting of the crystal.

An amorphous structure instead of a crystal as a ground state at zero temperature seems to be unlikely but the actual ion lattice type could be very sensitive to detailed conditions (Ichimaru, Iyetomi, and Mitake, 1983; Magierski and Heenen, 2002). However, Jog and Smith (1982) found that a phase composed of interpenetrating cubic lattices of different nuclei can be preferred in certain density regions.

\section{CONSTRAINTS ON THE EoS}

Models for the EoS can be constrained by different observables, which originate mainly from three different sources as follows: (1) laboratory measurements of nuclear properties and reactions, (2) theoretical ab initio calculations, and (3) observations in astronomy. These constraints can test different regions in the space of thermodynamic variables. They are, in the best case, independent of each other and different aspects of an EoS model can be checked; see, e.g., Klähn et al. (2006), Lattimer and Prakash (2007), Tsang et al. (2012), Lattimer and Lim (2013), Li and Han (2013), Horowitz et al. (2014), Lattimer and Steiner (2014), and Stone, Stone, and Moszkowski (2014) for discussions. Although we discuss a fair amount of constraints, keep in mind that these are usually limited to very restricted domains in the phase diagram (e.g., saturation properties are properties of symmetric matter, NSs are cold and do not explicitly probe the EoS at given density, etc.). Therefore, theoretical models are required to interpolate between or even extrapolate away from these constrained regions. While it is desirable that these models by themselves do not add further uncertainties one has to be cautiously aware that this is not necessarily the case.

Properties of nuclear matter are usually characterized by a number of parameters that are related to the leading contributions in an expansion of the energy per nucleon

$$
E\left(n_{B}, \delta\right)=E_{0}\left(n_{B}\right)+E_{\mathrm{sym}}\left(n_{B}\right) \delta^{2}+\mathcal{O}\left(\delta^{4}\right)
$$


in the isospin asymmetry $\delta=1-2 Y_{q}$. Both the energy per nucleon of symmetric matter

$$
E_{0}\left(n_{B}\right)=m_{\text {nuc }}-B_{\text {sat }}+\frac{1}{2} K x^{2}+\frac{1}{6} Q x^{3}+\cdots
$$

and the symmetry energy

$$
E_{\mathrm{sym}}\left(n_{B}\right)=J+L x+\frac{1}{2} K_{\mathrm{sym}} x^{2}+\cdots
$$

can be expanded close to nuclear saturation in the deviation $x=\left(n_{B}-n_{\text {sat }}\right) / 3 n_{\text {sat }}$ of the baryon density $n_{B}$ from the saturation density $n_{\text {sat }}$. Equations (34) and (35) define the binding energy at saturation $B_{\text {sat }}$, the incompressibility $K$, the skewness $Q$, the symmetry energy at saturation $J$, the symmetry energy slope parameter $L$, and the symmetry incompressibility $K_{\text {sym }}$. In general, nuclear matter parameters are strongly correlated among each other as well as to properties of nuclei and neutron stars; see, e.g., Klüpfel et al. (2009), Kortelainen et al. (2010), Lattimer and Lim (2013), and Lattimer and Steiner (2014). In recent years, many studies focused on obtaining constraints for the symmetry energy $E_{\text {sym }}$ and its density dependence; see, e.g., the contributions to the topical issue by $\mathrm{Li}$ et al. (2014). The most important constraints on the nuclear matter parameters and the EoS are discussed in more detail in the following sections.

\section{A. Terrestrial experiments}

\section{Systematics from nuclear masses and excitations}

The most basic and least ambiguous constraints for the EoS come from properties of nuclei, most notably nuclear masses (Audi, Wapstra, and Thibault, 2003; Wang et al., 2012) and density distributions (De Vries, De Jager, and De Vries, 1987; Angeli and Marinova, 2013). An extrapolation to infinite mass numbers yields the corresponding nuclear matter parameters. Besides the saturation point at saturation density of $n_{\text {sat }} \approx 0.15-0.16 \mathrm{fm}^{-3}$, and the corresponding value of the binding energy of $B_{\text {sat }} \approx 16 \mathrm{MeV}$, accurate constraints on the symmetry energy $E_{\text {sym }}$ and its density dependence are obtained. There exists a linear correlation between the symmetry energy at saturation $J$ and the slope parameter $L$ (Lattimer and Lim, 2013; Lattimer and Steiner, 2014). This correlation is very robust and validated in various theoretical approaches; see, e.g., Kortelainen et al. (2010), Fattoyev and Piekarewicz (2011), and Nazarewicz et al. (2014).

The aforementioned correlation is based on ground-state binding energies. Instead of ground-state binding energies, Danielewicz and Lee (2014) considered excitation energies to isobaric analog states and charge invariance to derive constraints for the symmetry energy. In a comprehensive analysis, Skyrme HF calculations were used to derive an acceptable region for $E_{\text {sym }}$ at densities from 0.04 to $0.16 \mathrm{fm}^{-3}$. They also extracted a constraint for $J$ and $L$ which significantly overlaps with the constraint from nuclear masses. At baryon densities $n_{B} \sim 0.105 \mathrm{fm}^{-3}$, the constraint of Danielewicz and Lee (2014) is the tightest, with an excellent accuracy of $\pm 1.2 \mathrm{MeV}$. For higher densities the constraint rapidly deteriorates, for lower densities it gets slightly worse. This "bottleneck" region was previously noted by Brown (2000),
Trippa, Colò, and Vigezzi (2008), and Roca-Maza, Brenna et al. (2013). Nuclear energy density functionals with different values of $J$ and $L$ that are fitted to binding energies of nuclei often show a crossing of their symmetry energies and/or their neutron matter EoS in this region. The corresponding density can be interpreted as an average value of the densities in finite nuclei. Danielewicz and Lee (2014) combined their analysis of isobaric analog states with measurements of skin thicknesses to arrive at tighter constraints of $J=30.2-33.7 \mathrm{MeV}$ and $L=35-70 \mathrm{MeV}$.

Obviously, constraints on the symmetry energy become tighter, if the experimental knowledge about binding energies is extended to very asymmetric nuclei. Many of the current high-precision mass measurements have been made possible by Penning-trap mass spectrometers or mass spectrometry with storage rings in combination with radioactive beams (Wolf et al., 2013). Binding energies of nuclei are also crucial for nucleosynthesis calculations and the location of the drip lines (Erler et al., 2012). They can also be used directly in the EoS of the outer crust of cold NSs; see, e.g., Baym, Pethick, and Sutherland (1971), Kreim et al. (2013), and Wolf et al. (2013), and Sec. V.A.1.

\section{Nuclear resonances}

Nuclear resonances in the form of collective excitations of finite nuclei contain important information about the isoscalar and isovector properties of the nucleon-nucleon interaction. For example, Paar et al. (2014) performed a global statistical analysis of experimental results for different collective excitations with emphasis on correlations between different observables. In addition to nuclear masses and charge radii, they considered the anti-analog giant dipole resonance, the isovector giant quadrupole resonance, the dipole polarizability of ${ }^{208} \mathrm{~Pb}$, and the pygmy dipole resonance transition strength in ${ }^{68} \mathrm{Ni}$. Employing a certain class of relativistic nuclear EDFs leads to tight constraints for $J=32.5 \pm 0.5 \mathrm{MeV}$ and $L=$ $49.9 \pm 4.7 \mathrm{MeV}$ and the crust-core transition density in NSs. Interestingly, the former values are fully compatible with the final results of Lattimer and Lim (2013) with $J=$ 29.0-32.7 MeV and $L=40.5-61.9 \mathrm{MeV}$ and Lattimer and Steiner (2014) with $L=44-66 \mathrm{MeV}$.

\section{a. Giant monopole resonance}

Constraints for the nuclear incompressibility $K$ can be deduced from fitting results of theoretical models to experimental data on the isoscalar giant monopole resonance (ISGMR), also called the breathing mode. However, it is perceived in the literature that the extraction of $K$ from ISGMR data is not unambiguous as it relates to the density dependence of the symmetry energy in the models (Piekarewicz, 2004; Shlomo, Kolomietz, and Colò, 2006; Sharma, 2009). For example, RMF models often obtain larger values for $K$ in the range of 250-270 MeV (Piekarewicz, 2004) than nonrelativistic models.

Recently, Khan and Margueron (2013) reanalyzed the problem of model dependencies. They showed that the data actually constrain the density-dependent incompressibility around the crossing density of $0.1 \mathrm{fm}^{-3}$, by using both relativistic and nonrelativistic EDFs. Therefore constraints 
on $K$ depend also on the skewness parameter $Q$ of the functional used to analyze the data. The situation is similar for the extraction of the symmetry energy from nuclear masses (cf. Sec. IV.A.1).

A very comprehensive list of theoretical calculations of $K$ from the literature was given by Stone, Stone, and Moszkowski (2014). In the same article, a reanalysis of the ISGMR was performed, based on a liquid-drop approach to the description of the vibrating nucleus. Interestingly, it was found that $K$ lies in the range of $250-315 \mathrm{MeV}$, which is significantly higher than the generally accepted values of $K=$ $248 \pm 8 \mathrm{MeV}$ (Piekarewicz, 2004) or $K=240 \pm 20 \mathrm{MeV}$ (Shlomo, Kolomietz, and Colò, 2006). They achieved consistency with the latter values provided the ratio of the surface to volume contributions $K_{\text {surf }} / K_{\text {vol }}$ in a leptodermous expansion is close to -1 , as predicted by a majority of mean-field models. However, in their analysis it seems that the experimental data favor a ratio different from -1 . The high values of $K$ are thus related to a different surface contribution to the ISGMR compared with other works employing mean-field models. Note that Stone, Stone, and Moszkowski (2014) were able to explain the ISGMR of tin isotopes, which was found by Piekarewicz (2010) to be a startling problem of nuclear structure.

\section{b. Giant dipole resonance}

The nuclear isovector giant dipole resonance (IVGDR) can be used to constrain the symmetry energy. From measured centroid energies for a liquid droplet model one obtains a correlation between the volume and surface part of the symmetry energy of finite nuclei (Lipparini and Stringari, 1989; Lattimer and Lim, 2013), which can be transformed into a correlation between $L$ and $J$. Trippa, Colò, and Vigezzi (2008) found that the IVGDR gives the tightest constraints on $E_{\text {sym }}$ around $n_{B}=0.1 \mathrm{fm}^{-3}$ with $23.3 \mathrm{MeV}<E_{\mathrm{sym}}\left(0.1 \mathrm{fm}^{-3}\right)<24.9 \mathrm{MeV}$, by analyzing the IVGDR by a variety of Skyrme models. Lattimer and Lim (2013) used different functional forms of $E_{\text {sym }}$ to extract the correlation between $L$ and $J$. Their results show a significant overlap with other constraints; see Fig. 1 in Lattimer and Steiner (2014).

The pygmy dipole resonance (PDR) at excitation energies much below the IVGDR is also sensitive to the symmetry energy (Klimkiewicz et al., 2007; Carbone et al., 2010). Reinhard and Nazarewicz (2010) and Daoutidis and Goriely (2011) argued that it is not possible to extract constraints on $J$ and $L$ from PDR strengths because their correlation with the symmetry energy is too weak. This was studied in more detail by Reinhard and Nazarewicz (2013) who found that the correlation between the accumulated low-energy strength and the symmetry energy "dramatically depends on the energy cutoff" used. Furthermore, they came to the conclusion that the low-energy dipole excitations cannot be interpreted in terms of a collective PDR mode.

\section{c. Electric dipole polarizability}

Tamii et al. (2011) reported a precise measurement of the electric dipole response of ${ }^{208} \mathrm{~Pb}$ from proton inelastic scattering. The extracted electric dipole polarizability $\alpha_{D}$ is correlated to the neutron skin thickness (Lipparini and Stringari, 1989; Reinhard and Nazarewicz, 2010; Piekarewicz et al., 2012), which in turn is correlated with $L$ (see Sec. IV.A.3). Using this two-step process, Lattimer and Lim (2013) obtained an anticorrelation between $L$ and $J$, with significant overlap with other constraints.

Recently Roca-Maza, Centelles et al. (2013) found that the product $\alpha_{D} J$ is much better correlated with the neutron skin thickness of ${ }^{208} \mathrm{~Pb}$ and $L$ than the polarizability $\alpha_{D}$ itself. After reanalyzing the experimental results of Tamii et al. (2011), Roca-Maza, Centelles et al. (2013) obtained a linear correlation between $J$ and $L$. Adopting a value of $J=$ $31 \pm 2 \mathrm{MeV}$, this resulted in $L=43 \pm(6)_{\text {expt }} \pm(8)_{\text {theor }} \pm$ (12) est MeV, where "expt" denotes experimental, and "theor" theoretical uncertainties, while "est" originates from the uncertainty in J. Tamii, von Neumann-Cosel, and Poltoratska (2014) obtained a linear correlation between $J$ and $L$. They pointed out that the difference to the anticorrelation found by Lattimer and Lim (2013) results from how they analyzed the data. Lattimer and Steiner (2014) revised the results of Lattimer and Lim (2013), taking the improved correlation of Roca-Maza, Centelles et al. (2013) into account. Roca-Maza et al. (2015) confirmed the correlation with additional data on other nuclei, slightly enlarging the interval for $J$ and $L$.

Zhang and Chen (2015) analyzed the data from Tamii et al. (2011) in yet another way. Instead of constraining nuclear matter properties at normal nuclear density, they showed that $\alpha_{D}$ of ${ }^{208} \mathrm{~Pb}$ puts stringent constraints on the symmetry energy, or almost equivalently the pure neutron matter EoS, at subsaturation densities significantly below $n_{\text {sat }}$. Their final results for the subsaturation EoS are consistent with the experimental constraints of Tsang et al. (2009) and Danielewicz and Lee (2014). In addition, they obtained agreement with various theoretical works for the neutron matter EoS, which are included in Fig. 6. The recent study of Hashimoto et al. (2015) determined the dipole polarizability of ${ }^{120} \mathrm{Sn}$, which is strongly correlated with that of ${ }^{208} \mathrm{~Pb}$, experimentally from proton inelastic scattering.

\section{Neutron skin thicknesses}

The density distributions of nucleons and their root-meansquare (rms) radii $\sqrt{\left\langle r_{i}^{2}\right\rangle}$ change rather smoothly for nuclei in the valley of stability when the mass number increases. However, the proton and neutron radii are not in general equal. Neutron-rich nuclei develop a neutron skin with thickness $\Delta r_{n p}=\sqrt{\left\langle r_{n}^{2}\right\rangle}-\sqrt{\left\langle r_{p}^{2}\right\rangle}$. The charge distributions and charge radii of many nuclei are well known experimentally, e.g., from elastic electron scattering or isotope shift measurements; see Angeli et al. (2009) and Angeli and Marinova (2013) and references therein. In contrast, neutron radii of nuclei and thus neutron skin thicknesses are much less precisely determined.

For the measurement of neutron radii of nuclei, experiments with particles that probe the neutron distribution with the help of the strong or weak interaction have to be utilized. Typical examples are proton scattering experiments (Ray, 1979; Ray and Hodgson, 1979; Klos et al., 2007; Terashima et al., 2008; 
Zenihiro et al., 2010), isovector giant dipole excitations by inelastic $\alpha$-particle scattering (Krasznahorkay et al., 1994), $\left({ }^{3} \mathrm{He}, t\right)$ charge exchange reactions (Krasznahorkay et al., 1999), the excitation of pygmy dipole resonances (Klimkiewicz et al., 2007), or the study of antiprotonic atoms (Trzcinska et al., 2001; Jastrzebski et al., 2004; Brown et al., 2007). Parity violation in elastic electron scattering is used in the lead radius experiment PREX at Jefferson Lab (Horowitz et al., 2001, 2012; Abrahamyan et al., 2012). In this type of approach the weak form factor of the nucleus is measured and it is primarily determined by the neutron density distribution. Unfortunately, the deduced neutron skin thickness of $0.302 \pm$ $0.175(\exp ) \pm 0.026$ (model) \pm 0.005 (strange) fm carries a large uncertainty. It is expected to diminish in future experimental runs. A noticeably smaller value of $\Delta r_{n p}=0.15 \pm$ 0.03 (stat) ${ }_{-0.03}^{+0.01}$ (sys) fm was recently reported from experiments of coherent pion photoproduction at the MAMI electron facility (Tarbert et al., 2014).

Brown (2000) found a strong correlation of the neutron skin thickness of ${ }^{208} \mathrm{~Pb}$ with the derivative $d E\left(n_{B}, \delta\right) /\left.d n_{B}\right|_{n_{B}=n_{0}, \delta=1}$ of the neutron matter EoS at a density $n_{0}=0.1 \mathrm{fm}^{-3}$ in nonrelativistic HF calculations with 18 different parametrizations of the Skyrme interaction. These observations triggered many theoretical and experimental studies to explore the relation of isospin-dependent properties of nuclei to the EoS, in particular, the density dependence of the nuclear symmetry energy $E_{\text {sym }}(n)$. An extension of the Skyrme HF calculations in similar studies of RMF models (Typel and Brown, 2001), general density functionals in the context of EFT (Furnstahl, 2002) or the droplet model (Warda et al., 2009) showed the same correlation, which can also be expressed as a correlation between $\Delta r_{n p}$ and the slope parameter $L$. More recent representations of the $\Delta r_{n p}-L$ and similar correlations of isospin-dependent properties can be found in Centelles et al. (2009), Chen et al. (2010), RocaMaza, Centelles et al. (2011), Gaidarov et al. (2012, 2014), Tsang et al. (2012), and Viñas et al. (2014a, 2014b). The consequences for the properties of NSs, such as radius or proton fraction, were studied by Horowitz and Piekarewicz (2001a, 2001b, 2002), Steiner et al. (2005), Todd-Rutel and Piekarewicz (2005), and Avancini et al. (2007a, 2007b, 2007c). The origin of the $\Delta r_{n p}-L$ correlation, its bulk, and surface contributions in nuclei and the relation to LandauMigdal parameters have been discussed by Dieperink et al. (2003), Centelles et al. (2010), and Warda et al. (2010). The neutron skin thickness provides a correlation between $L$ and $J$ as well and shows a decreasing of $L$ with increasing $J$, in contrast to other correlations of that type (Lattimer and Lim, 2013). The present data of the $\Delta r_{n p}-L$ correlation are derived only from mean-field calculations of nuclear structure. However, the neutron skin thickness could be modified by nucleon-nucleon correlations and clustering at the surface of the nucleus (Typel, 2014).

\section{Heavy-ion collisions}

The EoS of warm or hot, strongly interacting matter can be constrained in laboratory experiments with HICs. Depending on the beam energy, the impact parameter, the choice of observables, and the combination of projectile and target nuclei, very different conditions can be explored. In the early phase of almost central collisions of about $1 \mathrm{GeV}$, high densities of up to 4 times the nuclear saturation density and temperatures of about 40-50 MeV can be reached for a very short time (Blättel, Koch, and Mosel, 1993; Fuchs et al., 1997). In the later stages of a collision, more peripheral or less energetic reactions, properties of dilute matter at temperatures below the critical temperature of the liquid-gas phase transition (15-20 MeV) and subsaturation densities can be studied. We will not consider here ultrarelativistic HICs probing matter at very low baryon density and high temperatures. Since the physics of HICs is a large field on its own, we mention only the most important aspects relevant to this review.

There are fundamental differences between matter in HICs and in compact stars. Temperatures and densities can be similar to those in CCSNe, but matter in HICs is usually more isospin symmetric (cf. Sec. II.B.3). Furthermore, the fireball in a HIC has a finite size with a fixed number of nucleons that are not necessarily in thermal equilibrium. This limits, for example, the maximum mass number of nuclear clusters formed under these conditions. Matter in compact stars, which can be treated in the thermodynamic limit, has to be charge neutral, whereas there is a net charge in HICs fixed by the initial charge of the two colliding nuclei. In HICs Coulomb interactions are typically neglected because of the high kinetic energies. Characteristic time scales in HICs are of the order of a few $\mathrm{fm} / c$ and do not allow for equilibrium with respect to weak interactions. On the contrary, in catalyzed NSs full equilibrium is reached. In compact stars, weak equilibrium with respect to strangeness changing reactions is usually assumed, whereas the net strangeness in HICs is zero. These differences have to be taken into account when comparing astrophysical EoSs with constraints from HICs.

The analysis of HICs requires the comparison of measured data to rather complex theoretical simulations since a dynamical process has to be followed. These models are based on different approaches that aim to solve the relevant transport equations. On the one hand, a set of Boltzmann-type equations for the single quasiparticle distribution functions is considered; see, e.g., Danielewicz (1984a, 1984b). They can be derived consistently as an approximation of the nonequilibrium Kadanoff-Baym theory including collision and sometimes fluctuation terms (Bertsch and Das Gupta, 1988; Buss et al., 2012). On the other hand, simulations with molecular dynamics models in classical approximations, possibly including antisymmetrization effects, are also employed (Aichelin, 1991; Ono et al., 1992; Hartnack et al., 1998). One major challenge is to predict the distribution of observed particles and fragments reliably. In these models the EoS does not directly enter but the interactions between all particles which participate in the collision, as well as in-medium cross sections of the relevant reactions, which are usually parametrized in a convenient form ( $\mathrm{Li}$ and Chen, 2005). One important aspect is the momentum dependence of the interaction because particle momenta attain much larger values in HICs than in nuclei (Chen et al., 2014; Xu, Chen, and Li, 2015). Hydrodynamic descriptions (Welke et al., 1988; Gale et al., 1990; Huovinen and Ruuskanen, 2006; Gale, Jeon, and 
Schenke, 2013), which can make direct use of an EoS, are more appropriate for studying the evolution of the highdensity phase of a collision, in particular, in (ultra)relativistic HICs. However, one has to change to a different approach at later times when the system expands, the density drops, and fragments are formed.

There are several observables in HICs that are sensitive to particular features of in-medium interactions that determine the EoS at suprasaturation densities (Fuchs and Wolter, 2006). Not only nucleons but also mesons, such as pions or kaons, as well as light nuclei, e.g., ${ }^{2} \mathrm{H},{ }^{3} \mathrm{H},{ }^{3} \mathrm{He}$, and ${ }^{4} \mathrm{He}$ [see, e.g., Chajecki et al. (2014)], are valuable messengers for the properties of the medium at high and low densities, respectively.

The collective flow of nucleons exhibits a distinct azimuthal distribution (Welke et al., 1988), which can be characterized with coefficients in a Fourier analysis. The transverse flow in peripheral reactions seems to be mainly sensitive to the momentum dependence of the mean field. The elliptic flow, in contrast, depends strongly on the maximum compression that is reached and it is correlated with the stiffness of the EoS. The analysis of laboratory experiments in comparison with simulations indicates that the incompressibility of symmetric nuclear matter cannot be too high (Welke et al., 1988; Danielewicz, Lacey, and Lynch, 2002; Reisdorf et al., 2012; Le Fèvre et al., 2016) (see Sec. V.D.3 and Fig. 16).

The collision region with the highest densities is best studied with particles that are produced only there and interact weakly with the medium after their formation. Although being a rare probe due to their subthreshold production (Hartnack et al., 2012), kaons seem to be a good choice. Their observation in HICs points toward a rather low incompressibility $K$ with values below $250 \mathrm{MeV}$ (Fuchs et al., 2001; Sturm et al., 2001; Hartnack, Oeschler, and Aichelin, 2006). Consequences of these constraints from HICs on compact star properties were explored by Sagert, Tolos et al. (2012). For a discussion of the interplay between HICs and astrophysical data see, e.g., Aichelin and Schaffner-Bielich (2009).

In recent years, HIC experiments for constraining the EoS mainly focused on the isospin dynamics (Li, 2002; Baran et al., 2005; Li, Chen, and Ko, 2008; Di Toro et al., 2009; Wolter et al., 2009; Tsang et al., 2012; Cozma et al., 2013; Ademard et al., 2014; De Filippo and Pagano, 2014; Kohley and Yennello, 2014) in order to explore the properties of isospin asymmetric matter in more detail. Yield ratios of particle pairs with the same mass but different isospin, such as $n / p, \pi^{+} / \pi^{-}$(Xiao et al., 2014), or fragments ${ }^{3} \mathrm{H} /{ }^{3} \mathrm{He}$ (Yong et al., 2009) have been intensively investigated. The consideration of single or double ratios has the advantage that systematic experimental uncertainties are reduced and the sensitivity is increased. A possibility to amplify isospindependent effects is the comparison of collisions with different combinations of projectiles and targets with more or less neutron excess. For example, the isospin diffusion in the neck region of peripheral and midcentral collisions of ${ }^{112} \mathrm{Sn} /{ }^{124} \mathrm{Sn}$ nuclei is sensitive to the symmetry potential (Tsang et al., 2009, 2012).

The density dependence of the symmetry energy at moderate to high densities was studied with the help of $n / p$ ratios and their elliptic flow difference (Cozma, 2011; Russotto et al., 2011, 2013, 2014) as well as $\pi^{+} / \pi^{-}$ratios (Reisdorf et al., 2007). The analysis of the latter results within transport model simulations suggests a decrease of the symmetry energy at high densities, which is in conflict with most EoS models (Xiao et al., 2009; Xie et al., 2013). Also, the puzzling results for the effective mass splittings of nucleons in intermediate HICs (Zhang et al., 2014; Coupland et al., 2016) still need a satisfactory explanation (Kong et al., 2015). Only more accurate measurements will allow one to set tighter bounds on the symmetry energy at high densities.

Multifragmentation reactions probe conditions very similar to matter in CCSNe as illustrated in Fig. 4, where typical conditions for $\mathrm{CCSNe}$ and multifragmentation reactions are indicated; see the caption for details. In these reactions, a thermalized system of nuclear matter is formed that is characterized by subnuclear densities and temperatures of 3-8 MeV. The deexcitation of the system occurs via nuclear multifragmentation, i.e., breakup into many excited fragments and nucleons. For the theoretical description of such reactions, for instance the statistical multifragmentation model (SMM) is used that is presented in more detail in Sec. V.C. Statistical models accurately describe many characteristics of the nuclear fragments observed in the experiments: cluster multiplicities, charge and isotope distributions, various correlations, and

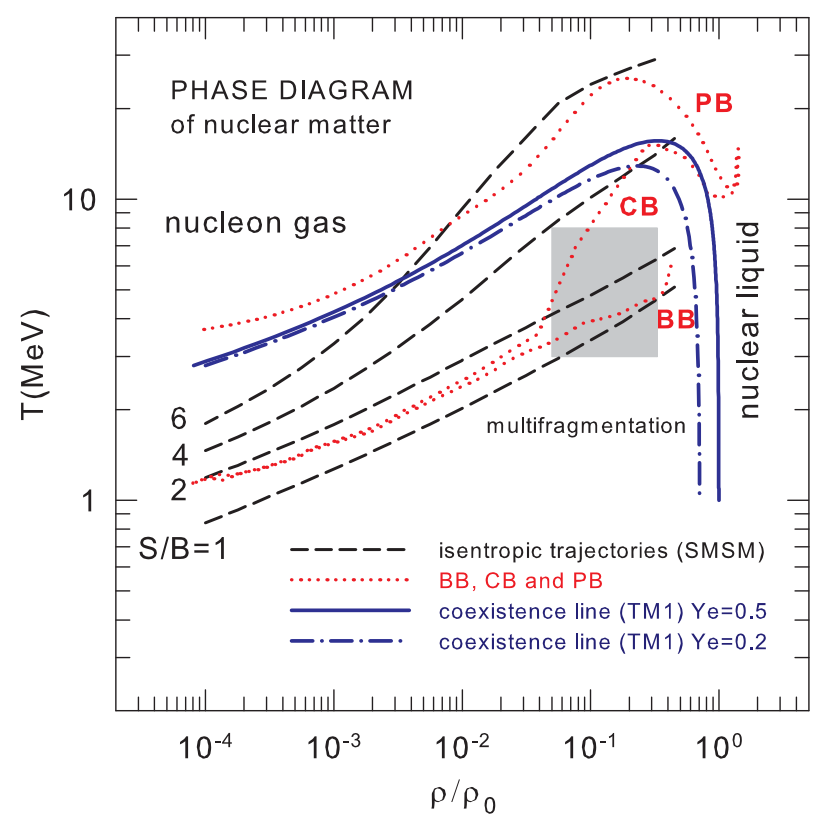

FIG. 4. Nuclear phase diagram in the temperature-baryon density plane. Solid and dash-dotted blue lines indicate boundaries of the liquid-gas coexistence region for symmetric and asymmetric matter calculated with TM1 interactions (Sugahara and Toki, 1994). The shaded area corresponds to typical conditions for nuclear multifragmentation reactions (Botvina and Mishustin, 2010). The dashed black lines are isentropic trajectories characterized by constant entropy per baryon, $s=1,2,4$, and 6 calculated with the statistical model for supernova matter (SMSM) (Botvina and Mishustin, 2010). The dotted red lines show results of a CCSN simulation from Sumiyoshi et al. (2005) just before bounce (BB), at core bounce (CB), and postbounce (PB). From Buyukcizmeci et al., 2013. 


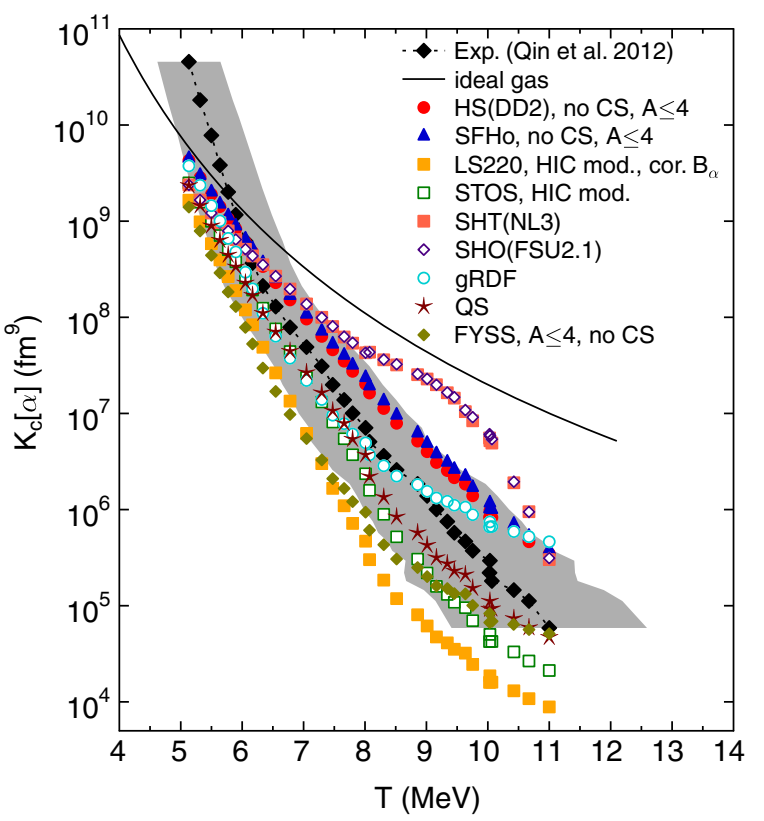

FIG. 5. Equilibrium constants of $\alpha$ particles extracted from HIC experiments (black diamonds) in comparison with those of various theoretical models, which are all adapted for the conditions in HICs, as far as possible. The gray band is the experimental uncertainty in the temperature determination. The black line shows the equilibrium constant of the ideal gas model. From Hempel et al., 2015.

other observables; see, e.g., Gross (1990), Bondorf et al. (1995), and Botvina and Mishustin (2010).

The observation of light nuclei, which are emitted in Fermi-energy HICs, allows one to determine the density and temperature of warm dilute matter from experiments (Kowalski et al., 2007; Natowitz et al., 2010; Wada et al., 2012). The derived symmetry energies of the clustered matter indicate an increase as compared to those obtained in model calculations of uniform matter that is assumed to be composed solely of nucleons. The thermodynamic conditions are similar to those in the neutrinosphere of CCSNe (Horowitz et al., 2014). From the observed yields of nucleons and clusters, it was possible to extract the in-medium binding energies and Mott points of light clusters (Hagel et al., 2012) with the help of chemical equilibrium constants (Qin et al., 2012). Hempel et al. (2015) refined the study of Qin et al. (2012), taking into account the differences between matter in HIC and CCSNe. Results for the equilibrium constant of the $\alpha$ particle are presented in Fig. 5. A comparison of many EoSs for warm dilute matter shows that simple NSE descriptions are not sufficient to reproduce experimental data (Hempel et al., 2015).

\section{B. Neutron matter calculations}

The simple isospin structure of pure neutron matter simplifies the nuclear interaction Hamiltonian, such that $a b$ initio calculations can be carried out more easily than in the case of general asymmetric nuclear matter. Calculations using the different many-body techniques introduced in Sec. III.B with well-calibrated interactions are available for a large range in densities; see, e.g., the review by Gandolfi, Gezerlis, and Carlson (2015) and references therein. They can serve as important constraints for the EoS models, discussed in Sec. V, although their results are not directly applicable to astrophysical objects.

At very low densities, neutron matter is dominated by $s$ wave interactions with a large scattering length $a=-18.5 \mathrm{fm}$, indicating that the two-neutron system is almost bound. Neutron matter at these densities is close to the unitary limit, explored experimentally for cold fermionic atoms (Ho and Mueller, 2004; Ho and Zahariev, 2004).

At intermediate densities, up to roughly nuclear matter saturation density, and at higher densities, relevant for NSs, many different calculations from $a b$ initio methods exist. We mention some calculations, without claiming completeness for the list below. Seminal results are the variational calculations by Friedman and Pandharipande (1981) and Akmal, Pandharipande, and Ravenhall (1998) using the Urbana and Argonne nuclear two- and three-body forces. BHF calculations have been reported for instance by Baldo, Bombaci, and Burgio (1997) and Zhou et al. (2004), and DBHF results by van Dalen, Fuchs, and Faessler (2004), Krastev and Sammarruca (2006), and Sammarruca et al. (2012). Rios, Polls, and Vidaña (2009) compared SCGF calculations for thermodynamic properties of hot neutron matter with the corresponding BHF calculations. Carbone (2014) obtained SCGF results including an effective three-nucleon force for finite temperature and extrapolated them to vanishing temperature. Horowitz and Schwenk (2006b, 2006c) applied the virial expansion to dilute neutron matter at nonzero temperature. An early application of the virial expansion was the description of a neutron gas in supernovae by Buchler and Coon (1977) using the soft-core Reid potential. QMC calculations for zero temperature neutron matter using different versions of the Argonne and Urbana nuclear potentials have been presented by Carlson et al. (2003), Gandolfi et al. (2009), Wlazłowski and Magierski (2011), and Gandolfi, Carlson, and Reddy (2012). Recent QMC (Gezerlis et al., 2013, 2014; Roggero, Mukherjee, and Pederiva, 2014; Wlazłowski et al., 2014) and coupled cluster (Baardsen et al., 2013; Hagen et al., 2014) calculations employ chiral potentials. Neutron matter is particularly interesting for chiral forces, since only a few LECs accompanying the contact terms are involved up to next-to-next-to-next-to leading order $\left(\mathrm{N}^{3} \mathrm{LO}\right)$ including three- and four-nucleon forces. In addition, at least up to roughly saturation density, the MBPT results with RG evolved and unevolved chiral forces are in very good agreement, showing that neutron matter in this range behaves perturbatively to a very good approximation; see, e.g., Tolos, Friman, and Schwenk (2008) and Krüger et al. (2013). Comparison with QMC calculations corroborates the perturbative nature of neutron matter at these densities (Gezerlis et al., 2014). Calculations of neutron matter with chiral forces can be found in Hebeler and Schwenk (2010, 2014), Krüger et al. (2013), and Tews et al. (2013). In-medium $\chi \mathrm{EFT}$ following different power counting schemes was applied to neutron matter at zero and nonzero temperature by Lacour, Oller, and Meißner (2011), Fiorilla, Kaiser, and Weise (2012), and Drischler, Soma, and Schwenk (2014). 
In Fig. 6 we show the energy per baryon of pure neutron matter as a function of baryon number density as obtained in a few of the different approaches cited previously. The yellow region corresponds to the AFQMC calculations of Gandolfi et al. (2009) and Gandolfi, Carlson, and Reddy (2012) with the Argonne two-body potential. The width of the band indicates uncertainties related to the different phenomenological threebody forces. The AFQMC results of Wlazłowski et al. (2014) with chiral forces (green lines and symbols) including only two-nucleon interactions (2NF) and those including a threenucleon force $(2 \mathrm{NF}+3 \mathrm{NF})$ are in good agreement with the former ones. The variational results of Friedman and Pandharipande (1981) gave lower values than Akmal, Pandharipande, and Ravenhall (1998) and lie at the lower boundary of the AFQMC calculations. The width of MBPT results by Hebeler et al. (2013) shows mainly uncertainties in the three-nucleon forces employed. The constraint derived by Krüger et al. (2013) at saturation density comparing different chiral forces and cutoff schemes within a MBPT calculation is shown as a vertical error bar. Figure 6 also displays the $\chi \mathrm{EFT}$ results by Fiorilla, Kaiser, and Weise (2012) and BHF calculations from Vidaña et al. (2010). The latter use Argonne plus phenomenological three-body potentials.

We address two points concerning these different results. First, three-nucleon forces (and potentially more, depending on the resolution scale and density) are important in neutron matter and add substantial repulsion at and above saturation density. This is seen, for example, by comparing QMC calculations with and without three-nucleon forces. Second, all results, with phenomenological or chiral forces, applying different manybody techniques, are in reasonable agreement up to saturation density. This shows that the ab initio many-body calculations represent a reliable constraint on the EoS of neutron matter up to nuclear densities (see Sec. V.D.3 and Fig. 16).

The situation is different for calculations of symmetric nuclear matter. They have been a cornerstone for many-body methods for decades. However, the empirical saturation point is difficult to obtain. In addition, symmetric matter is unstable with respect to cluster formation at densities below saturation which is strongly temperature dependent and leads to an

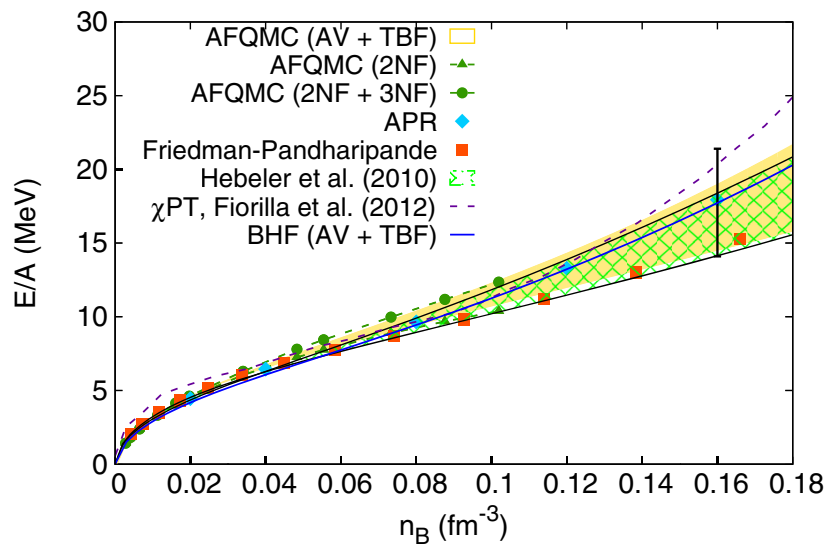

FIG. 6. Comparison of results for the energy per baryon of neutron matter at $T=0 \mathrm{MeV}$ from different $a b$ initio approaches. The vertical bar represents the range given at saturation density in Krüger et al. (2013). For details see text. increase of the binding energy. Therefore theoretical manybody calculations of symmetric matter are not as reliable as for neutron matter and cannot serve as a constraint on the EoS at present. Instead phenomenological models are adjusted to the empirical properties of symmetric matter.

\section{Astrophysical observations}

\section{Neutron star masses and radii}

Presently, the main astrophysical constraint stems from the measurements of two very massive NSs in NS-white dwarf systems which have been reported with unprecedented high precision. For the first binary system, the determination is based on Shapiro delay, a general relativistic effect (Demorest et al., 2010). It yields a mass of $1.928 \pm 0.017 M_{\odot}$ (Fonseca et al., 2016). In the second case a well-known structure model for the white dwarf is combined with the analysis of orbital data to obtain a mass of $2.01 \pm 0.04 M_{\odot}$ for the NS (Antoniadis et al., 2013). There are indications of even more massive NSs in black widow and redback systems (van Kerkwijk, Breton, and Kulkarni, 2011; Romani et al., 2012; Kaplan et al., 2013). In these cases, the pulsar is accompanied by a low-mass companion of a few $0.001 M_{\odot}$ (black widows) or near $0.2 M_{\odot}$ (redbacks), which is bloated and strongly irradiated by the pulsar. However, the analysis of these systems is much more model dependent than for NS-white dwarf systems. In particular, the companion's light curve has to be modeled inducing large uncertainties in the mass determination. Although the most probable mass for the NS indicates a very massive object, the results do not yet reach the same reliability as the mass determinations of Antoniadis et al. (2013) and Fonseca et al. (2016). This also holds for the NS in the eclipsing $\mathrm{X}$-ray binary Vela X-1, where a high mass of $2.12 \pm 0.16 M_{\odot}$ has been reported by Falanga et al. (2015).

Smaller NS masses have been measured in various binary systems; see Lattimer (2012) for a recent compilation. In some cases masses have been derived very precisely from the orbital parameters of the system without much model dependence in the analysis. Particularly precise measurements have been performed for several binary NS systems giving masses close to the canonical value of $1.4 M_{\odot}$.

At the other end, the lowest NS masses could be interesting for constraining the EoS via their formation history. Originally, Podsiadlowski et al. (2005) suggested to consider pulsar B in the double pulsar system J0737-3039, with a very low and precisely measured mass of $1.2489 \pm 0.0007 M_{\odot}$. If it originates from the collapse of a progenitor star with $\mathrm{O}-\mathrm{Ne}-\mathrm{Mg}$ core and the loss of matter during the formation of the NS is negligible, the baryon number, or equivalently the corresponding baryon mass $M_{B}$ for the NS, is strongly constrained from the properties of the white dwarf progenitor. Its mass was determined to be $1.366 M_{\odot} \leq M_{B} \leq 1.375 M_{\odot}$ (Podsiadlowski et al., 2005), assuming a stationary nonrotating object. Kitaura, Janka, and Hillebrandt (2006) concluded on a slightly smaller but similar mass of $M_{B}=$ $1.36 \pm 0.002 M_{\odot}$ from simulations of an electron-capture supernova. A similar system J1756-2251 was recently observed with a slightly lower gravitational mass of $1.230 \pm$ $0.007 M_{\odot}$ for the pulsar with the lower mass (Ferdman et al., 
2014). The constraint on the EoS arising from the relation between gravitational and baryon mass of these low-mass NSs depends strongly on assumptions. First, there is no complete consensus about the formation history of these systems and the origin from a $\mathrm{O}-\mathrm{Ne}-\mathrm{Mg}$ electron-capture supernova is not confirmed (Tauris et al., 2013). Second, already a possible baryon loss of $1 \%$ during the formation of the compact star broadens the corresponding baryon mass region by increasing it by roughly a factor of 2 . This effect is included in the constraints derived by Kitaura, Janka, and Hillebrandt (2006) but only for two particular EoSs.

The ultimate constraint on the EoS is a determination of radius and mass of the same object; see, e.g., Özel and Psaltis (2009), Read et al. (2009), Özel, Baym, and Güver (2010), and Steiner, Lattimer, and Brown (2013). Recently, Sotani et al. (2014) discussed how for low-mass NSs this could be translated into a constraint for a particular combination of $K$ and $L$. Currently, radius observations are much more model dependent than mass measurements, largely because radius measurements are much more indirect. Possible sources of systematic error include the composition of the atmosphere, the strength of the magnetic field, the distance to the source, interstellar extinction, residual accretion in binaries, brightness variations over the surface, and the effects of rotation in sources with unknown spin frequencies; see Miller (2013) and Potekhin (2014) for details. The importance of uncertainties in determining the radius depends on the type of the object observed. Currently radii are extracted from four different types of sources as follows:

(1) Isolated neutron stars (INSs). For INSs it is extremely difficult to determine the distance, the magnetic field, and the composition of the atmosphere inducing altogether very large uncertainties on the radius determinations from these sources; see, e.g., the discussion by Potekhin (2014).

(2) Quiescent X-ray transients (QXTs) in low-mass X-ray binaries. The thermal emission from the surface of the NS can be observed in the quiescent phase, i.e., when the accretion of matter from the companion is absent or at least strongly reduced. They are promising sources for radii determinations, since the magnetic field of QXTs is low due to the accretion of matter. In addition, the atmosphere is likely to be composed of light elements ( $\mathrm{H}$ or possible $\mathrm{He}$ ) and if they are situated in globular clusters, the distance is well known. Recent radius determinations from QXTs are shown in Fig. 7 as SL13 (Steiner, Lattimer, and Brown, 2013), GS13 and GS13m (Guillot et al., 2013), and GR14 (Guillot and Rutledge, 2014). Although promising sources, the results are still subject to many uncertainties. For instance, there has been recent discussion about the NS's atmospheric composition in quiescent low-mass $\mathrm{X}$-ray binaries (qLMXBs) in the globular cluster NG 6397. Guillot et al. (2013) and Guillot and Rutledge (2014) favored an unmagnetized hydrogen atmosphere and obtained a small radius of $R_{1.4}=9.4 \pm 1.2 \mathrm{~km}$ (90\% confidence level) for a $1.4 M_{\odot}$ NS (Guillot and Rutledge, 2014). Heinke et al. (2014) argued that a helium atmosphere

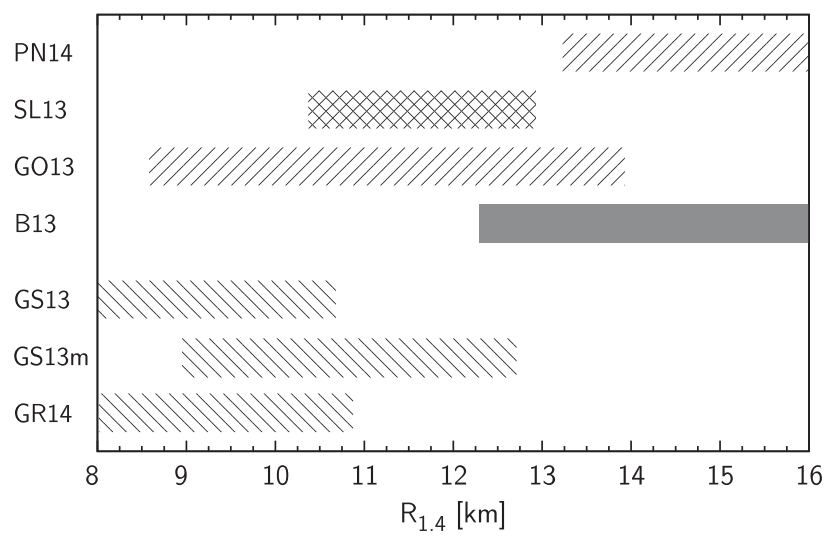

FIG. 7. Summary of recent NS radius estimations from observations for a star with the canonical mass of $1.4 M_{\odot}$. Shown are $2 \sigma$ error bars. For details see Table 2 of Fortin et al. (2015) and the text. From M. Fortin.

is more probable which leads to approximately $2 \mathrm{~km}$ larger radii. GS13m therefore shows the result of Guillot et al. (2013) upon excluding the qLMXB in NGC 6397.

(3) Bursting NSs (BNSs). From these objects very powerful photospheric radius expansion bursts are observed. Similar to QXTs, they have low magnetic fields and a light element atmosphere and, if situated in globular clusters, the distance can be well determined. The main uncertainties arise here from the modeling of the photospheric burst and no consensus has yet been reached; see, e.g., Galloway and Lampe (2012), Özel, Gould, and Güver (2012), Güver and Özel (2013), Steiner, Lattimer, and Brown (2013), and Poutanen et al. (2014). Recent radius determinations from BNSs are shown in Fig. 7; PN14 from Poutanen et al. (2014), GO13 from Güver and Özel (2013), and SL13 from Steiner, Lattimer, and Brown (2013).

(4) For rotation-powered millisecond pulsars radii can be determined from the shape of the $\mathrm{x}$-ray pulses. They are interesting, in particular, if the mass is known from radio observations. The result of Verbiest et al. (2008) and Bogdanov (2013) for J0437-4715 is shown in Fig. 7 (B13). Although with large uncertainties, the possible mass-radius region of neutron star XTE J1807-294 has been derived by Leahy, Morsink, and Chou (2011).

QXTs as well as BNSs are likely to rotate at a frequency of a few hundred of $\mathrm{Hz}$, inducing a non-negligible rotational deformation that complicates the analysis of the $\mathrm{x}$-ray spectra. The latter effect is expected to affect the radii by roughly $10 \%$ (Poutanen et al., 2014; Bauböck et al., 2015). Özel et al. (2016) included this rotational correction in a combined analysis of observed QXTs and BNSs. This common analysis of 12 sources statistically reduces the error on the final result for the radius obtained $R_{1.5}=10.1-11.1 \mathrm{~km}$.

In conclusion, present radius determinations are subject to many assumptions and uncertainties; see also the discussion by Potekhin (2014) and Fortin et al. (2015). Currently, they cannot provide as stringent constraints as some of the mass measurements. However, much observational efforts are 
directed to NS radius measurements. Future high-precision $\mathrm{x}$-ray astronomy, such as proposed by the projects NICER, ATHENA + or LOFT, and the gravitational wave signal of NS mergers expected for the near future (see Sec. VI.A) would help substantially to constrain radii and consequently the EoS of NS matter.

Another interesting possibility to determine a relation between mass and radius of a NS would be the observation of the gravitational redshift at the NS surface. Cottam, Paerels, and Mendez (2002) deduced a value of $z=0.35$ from narrow absorption lines in the spectra of X-ray bursts from EXO 0748676. However, this observation could not be confirmed later (Cottam et al., 2008). In addition, the rotation frequency of the source was measured to be of the order of 400-500 $\mathrm{Hz}$. Therefore, one expects not narrow but wider lines. As a consequence Lin et al. (2010) concluded that these spectral lines do not actually originate from the surface. However, a more recent study by the same group suggests that line profiles from rotating NSs might actually be narrower than initially predicted (Bauböck, Psaltis, and Özel, 2013).

\section{Neutron star cooling and rotation}

While computing mass and radius of a NS requires only a known relation between total pressure and total energy density [see Eq. (36)], the cooling of NSs depends on a detailed description of the interior composition which determines the heat transport and amount of neutrino emission. The occurrence of superfluid states barely influences the structure of a NS but has great impact on cooling. First, pair breaking and formation are important neutrino emission channels. At a later stage, for temperatures below the corresponding critical temperature, the related pairing gaps suppress the emission of neutrinos and reduce the heat capacity and thermal conductivity (Blaschke, Grigorian, and Voskresensky, 2004; Yakovlev and Pethick, 2004; Page, Geppert, and Weber, 2006). NS cooling and the description of superfluid phases have been reviewed by Weber (1999), Yakovlev and Pethick (2004), and Potekhin, Pons, and Page (2015). Although cooling calculations face many difficulties due to a large number of not precisely known quantities, the direct cooling observation of the young, only about $330 \mathrm{yr}$ old NS in Cassiopeia A (Heinke and Ho, 2010) over a period of $10 \mathrm{yr}$ promises to give direct insight into its composition (Page et al., 2011; Shternin et al., 2011; Blaschke et al., 2012; Sedrakian, 2013). A recent analysis of Chandra observations suggests that the initially reported fast cooling has to be considered with caution due to involved statistical uncertainties (Posselt et al., 2013) and possible instrumental problems (Elshamouty et al., 2013). Nevertheless, the theoretical work has demonstrated the strong impact precise cooling observations can have.

Neutron star rotation rates can be determined precisely from pulsar observations. Theoretically, slowly rotating stars can be described in the Hartle and Thorne (1968) approximation; see, e.g., Weber (1999). Numerically precise solutions (Nozawa et al., 1998) can be obtained up to the mass shedding limit, the Kepler frequency; see Friedman and Stergioulas (2013). The value of the Kepler frequency depends on the EoS and an observed frequency above the Kepler limit for a given EoS would clearly exclude the underlying model. Currently observed rotation rates (Hessels et al., 2006; Kaaret et al., 2007) with a maximum of $716 \mathrm{~Hz}$ do not put relevant constraints on the EoS (Haensel et al., 2009), but this could change if more rapidly rotating stars are observed in the future. Other astrophysical observations can be used to derive EoS constraints, e.g., quasiperiodic oscillations in soft-gamma-ray repeaters (Steiner and Watts, 2009; Sotani et al., 2012). However, the modeling of these events is complicated and often relies on additional model assumptions.

\section{Summary of constraints on the symmetry energy}

Besides the constraints discussed, further constraints on the symmetry energy at saturation and on the slope parameter have been collected in the literature in Tsang et al. (2012), Lattimer and Lim (2013), Li and Han (2013), and Lattimer and Steiner (2014). Extending these data collections, the compilations in Fig. 8 depict the probability distributions of $J$ and $L$ values, respectively. For simplicity, the probability distributions are assumed to be of Gaussian form with an area normalized to 1 . They are centered at the obtained values for $J$ and $L$ with widths that are given by the errors of the individual
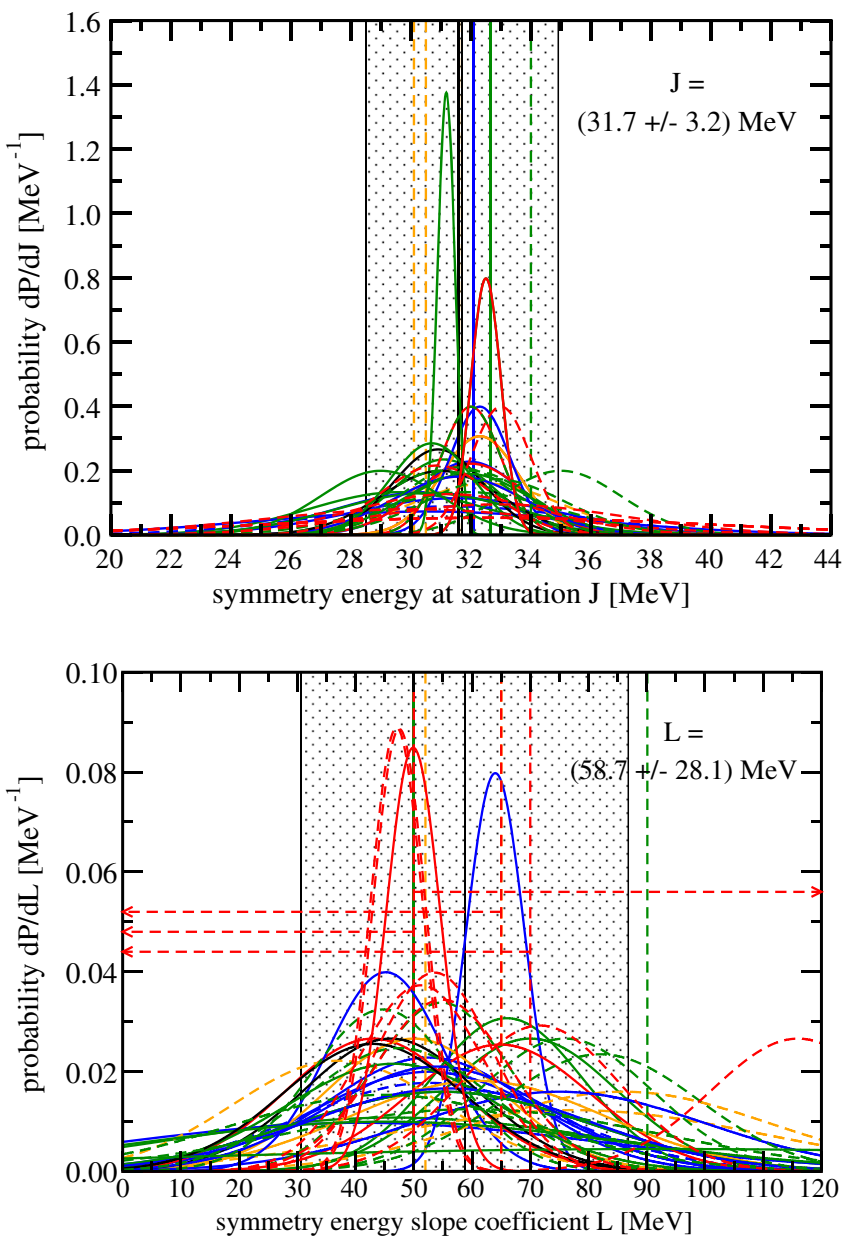

FIG. 8. Probability distribution of the symmetry energies at saturation $J$ (top panel) and of the symmetry energy slope parameter $L$ (bottom panel) from various studies. See text for details. 
studies. Dashed vertical lines are used if no uncertainty is available. Allowed ranges with upper or lower bounds are indicated by arrows. In general, nuclear matter parameters such as $J$ and $L$ and their errors are correlated in models that are used in the analysis of experimental and observational

TABLE II. Sources of the data for the symmetry energies at saturation $J$ and slope parameters $L$ used in Fig. 8, including the color code.

\begin{tabular}{|c|c|}
\hline Type of constraint & References \\
\hline $\begin{array}{l}\text { Systematic of nuclear } \\
\text { masses (solid green lines) }\end{array}$ & $\begin{array}{l}\text { Myers and Swiatecki (1996), } \\
\text { Danielewicz (2003), } \\
\text { Mukhopadhyay and Basu } \\
\text { (2007), } \\
\text { Klüpfel et al. (2009), } \\
\text { Kortelainen et al. (2010), } \\
\text { Liu et al. (2010), } \\
\text { Möller et al. (2012), } \\
\text { Lattimer and Lim (2013), } \\
\text { Wang, Ou, and Liu (2013), } \\
\text { and Viñas et al. (2014b) }\end{array}$ \\
\hline $\begin{array}{l}\text { Neutron skin data and other } \\
\text { nuclear structure } \\
\text { information } \\
\text { (solid blue lines) }\end{array}$ & $\begin{array}{l}\text { Centelles et al. (2009), } \\
\text { Warda et al. (2009), } \\
\text { Chen et al. (2010) } \\
\text { Chen (2011), Agrawal, De, } \\
\text { and Samaddar (2012), } \\
\text { Dong et al. (2012), Zhang } \\
\text { and Chen (2013), Wang and Li } \\
\text { (2013), Danielewicz and Lee } \\
\text { (2014), and Viñas et al. } \\
\text { (2014b) }\end{array}$ \\
\hline $\begin{array}{l}\text { Nuclear resonances } \\
\text { (solid red lines) }\end{array}$ & $\begin{array}{l}\text { Klimkiewicz et al. (2007), } \\
\text { Carbone et al. (2010), Roca- } \\
\text { Maza, Brenna et al. (2013), } \\
\text { Colo, Garg, and Sagawa } \\
\text { (2014), and Paar et al. (2014) }\end{array}$ \\
\hline $\begin{array}{l}\text { Dipole polarizability of nuclei } \\
\text { (solid black lines) }\end{array}$ & $\begin{array}{l}\text { Roca-Maza, Centelles et al. } \\
\text { (2013) and Tamii, von } \\
\text { Neumann-Cosel, and } \\
\text { Poltoratska (2014) }\end{array}$ \\
\hline $\begin{array}{l}\alpha \text { and } \beta \text { decay of nuclei } \\
\text { (solid orange lines) }\end{array}$ & $\begin{array}{l}\text { Dong et al. (2013) and Dong, } \\
\text { Zuo, and } \mathrm{Gu}(2013)\end{array}$ \\
\hline $\begin{array}{l}\text { Global nucleon optical } \\
\text { potentials (dashed blue } \\
\text { lines) }\end{array}$ & $\mathrm{Xu}, \mathrm{Li}$, and Chen (2010) \\
\hline $\begin{array}{l}\text { Heavy-ion collisions } \\
\quad \text { (dashed orange lines) }\end{array}$ & $\begin{array}{l}\text { Tsang et al. (2004, 2009), Chen, } \\
\text { Ko, and Li (2005a, 2005b), Li } \\
\text { and Chen (2005), Shetty, } \\
\text { Yennello, and Souliotis (2007), } \\
\text { Sun et al. (2010), and Kohley } \\
\text { et al. (2010) }\end{array}$ \\
\hline $\begin{array}{l}\text { Theoretical calculations } \\
\text { (dashed green lines) }\end{array}$ & $\begin{array}{l}\text { Erler, Klüpfel, and Reinhard } \\
\text { (2010), Gandolfi, Carlson, and } \\
\text { Reddy (2012), Fiorilla, Kaiser, } \\
\text { and Weise (2012), Erler } \text { et al. } \\
\text { (2013), Hebeler } \text { et al. (2013), } \\
\text { Krüger et al. (2013), and } \\
\text { Nazarewicz et al. (2014) }\end{array}$ \\
\hline $\begin{array}{l}\text { Properties of neutron stars } \\
\text { (dashed red lines) }\end{array}$ & $\begin{array}{l}\text { Newton and Li (2009), Steiner, } \\
\text { Lattimer, and Brown (2010, } \\
\text { 2013), Gearheart et al. (2011), } \\
\text { Steiner and Gandolfi (2012), } \\
\text { Wen, Newton, and Li (2012), } \\
\text { Vidaña (2012), Sotani et al. } \\
\text { (2013a, 2013b), and Lattimer } \\
\text { and Lim (2013) }\end{array}$ \\
\hline
\end{tabular}

data. Since these correlations are rarely specified in the literature (Lattimer and Lim, 2013), we treat $J$ and $L$ as independent quantities. The origin of the constraints is encoded in Fig. 8 by colors; see Table II. Averaging over this selection of results (excluding upper and lower bounds) we find $J=31.7 \pm 3.2 \mathrm{MeV}$ and $L=58.7 \pm 28.1 \mathrm{MeV}$ with an error for $L$ that is considerably larger than that for $J$.

\section{MODELING THE EOS}

In this section, we describe models for the EoS, i.e., particular realizations of the formal approaches introduced in Sec. III. It is evident that the requirements on the EoS are different depending on the astrophysical situation to which they are applied (see Sec. II.B). We place emphasis on the general purpose EoSs that cover the full thermodynamic parameter range in $T, n_{B}$, and $Y_{q}$. An overview of the currently available ones is presented in Sec. V.D. The reason for this is twofold. First, there is a plethora of different EoSs available in the literature applicable in a particular context, especially for cold $\beta$-equilibrated NSs. To list all available models, including variations of free parameters such as coupling constants in the phenomenological models, would be fruitless. Second, excellent reviews already exist; see, e.g., Lattimer and Prakash (2007), Baldo and Burgio (2012), and Lattimer (2012). We therefore discuss only some selected aspects of EoSs of cold $\beta$-equilibrated neutron stars (see Sec. V.A) and of EoSs describing homogeneous matter at finite temperatures suitable for describing hydrostatic PNSs (see Sec. V.B). Section V.C gives a few representative examples of EoSs that describe clusterization and nuclear statistical ensembles at finite temperatures but that are restricted to subsaturation densities.

Almost exclusively, phenomenological models have been used up to now in the context of astrophysical applications due to the computational complexity in the description of clustered matter. This concerns the NS crust discussed in Sec. V.A.1, the NSE-type EoSs in Sec. V.C, and, in particular, the general purpose EoSs discussed in Sec. V.D. The most advanced descriptions of dense matter are found for particular conditions. In fact, the $a b$ initio approaches discussed in Sec. III, if not restricted to pure neutron matter, have been applied only to homogeneous nuclear matter at various neutron-to-proton ratios with an interpolation to obtain the $\beta$-equilibrated NS EoS. Some $a b$ initio calculations exist at finite temperature as some of the methods, for instance, SCGF, are easier to treat at nonzero temperature, but the composition is fixed and matter is homogeneous (see Sec. V.B for some examples). It is desirable that in the future reliable approaches will be developed to describe strongly interacting matter for all relevant conditions needed in compact star astrophysics. A first step is that the information obtained from $a b$ initio neutron matter calculations, experiments, and NS observations is fully exploited to constrain the general purpose models.

\section{A. Neutron star EoS}

The physics of NSs has been discussed in detail in several works (Baym and Pethick, 1975, 1979; Glendenning, 1997; Heiselberg and Pandharipande, 2000; Lattimer and Prakash, 


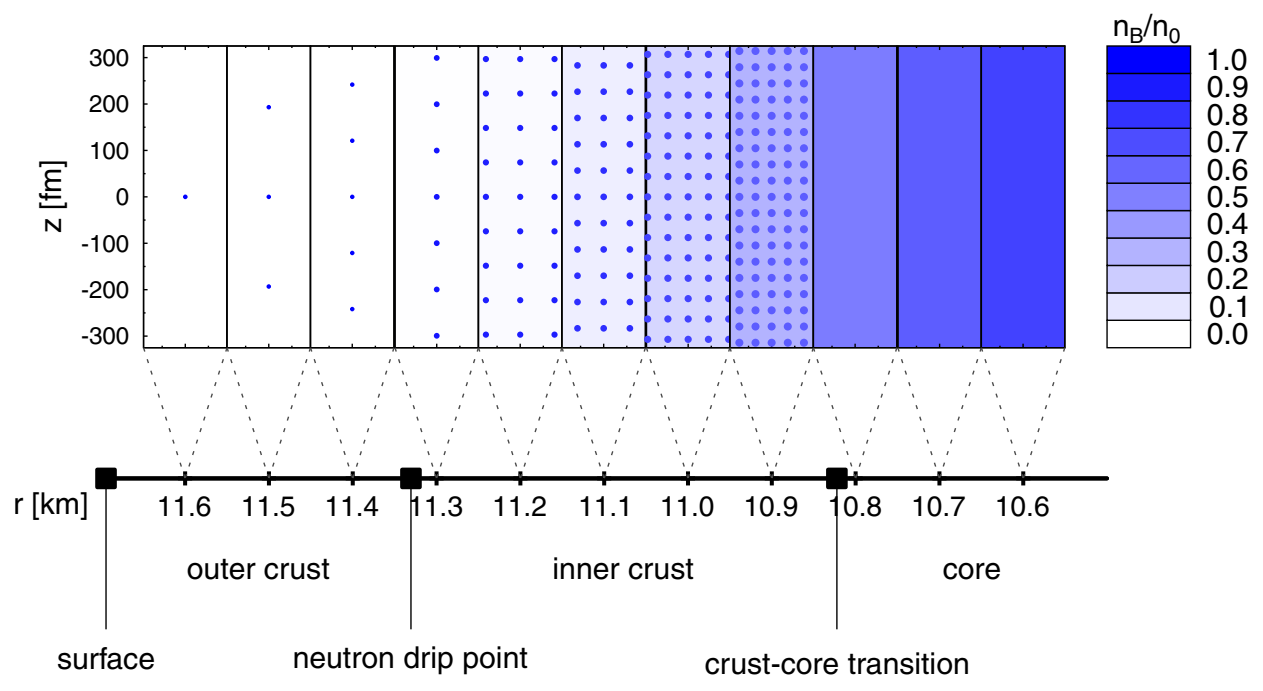

FIG. 9. Graphical representation of the structure and composition of the crust of a $1.44 M_{\odot}$ NS. Each subpanel shows in color coding (see legend at right) the mean local density of the nucleons, for the position in the NS as indicated in the bottom part of the figure. The illustration uses the SLy4 EDF, the EoS of Ruester, Hempel, and Schaffner-Bielich (2006) for the outer crust, and the EoS of Douchin and Haensel (2001) for the inner crust and the core.

2001, 2004, 2007, 2011; Sedrakian, 2007; Chamel and Haensel, 2008; Potekhin, 2010). NS matter is charge neutral and can be considered as cold $(T=0)$ and in general in $\beta$ equilibrium. The EoS thus depends only on one state variable, which can be conveniently chosen. For example, this can be the baryon number density. The EoS entirely determines global properties of stationary NSs, such as masses and radii. For nonrotating stars with negligible magnetic field, they are found by solving the following TolmanOppenheimer-Volkoff (TOV) equations (Oppenheimer and Volkoff, 1939; Tolman, 1939):

$$
\begin{aligned}
& \frac{d P(r)}{d r}=-G \frac{[\epsilon(r)+P(r)]\left[M(r)+4 \pi r^{3} P(r)\right]}{r^{2}[1-2 G M(r) / r]}, \\
& \frac{d M(r)}{d r}=4 \pi \epsilon(r) r^{2},
\end{aligned}
$$

relating the gravitational mass of the star $M$ inside a radius $r$ to pressure $P$ and energy density $\epsilon$. The EoS in terms of $P$ and $\epsilon$ closes the system of equations. Despite the assumptions of zero temperature and $\beta$ equilibrium, the calculation of the NS EoS is not a trivial task, particularly if microscopic methods are applied; see, e.g., Baldo, Bombaci, and Burgio (1997), Vidaña et al. (2010), Schulze and Rijken (2011), and Baldo and Burgio (2012). The domain of validity of some $a b$ initio methods is restricted to rather low densities not exceeding nuclear saturation density substantially. The size of higherorder contributions in systematic expansions, such as $\chi$ EFT approaches, increases with density, and the composition of matter at suprasaturation densities is rather uncertain (see Sec. V.A.2). In order to cover the whole density range required to describe NSs, microscopic EoSs can be extended at high densities with generic parametrizations, such as piecewise polytropes. Thereby the uncertainty of the NS mass-radius relation and the dependence on the model parameters can be explored (Hebeler et al., 2013).

\section{Neutron star crust EoSs and unified neutron star EoSs}

For mass densities below about $10^{4} \mathrm{~g} / \mathrm{cm}^{3}$, an atmosphere of partially ionized atoms and electrons forms the outer part of a NS with an EoS given by Feynman, Metropolis, and Teller (1949), Rotondo et al. (2011), and de Carvalho et al. (2014). At higher densities, the spatial region that is made up of inhomogeneous nucleonic matter and electrons not bound to nuclei in $\beta$ equilibrium is called the crust. It can be divided into an outer crust with a plasma of nuclei and electrons as degrees of freedom and an inner crust where also unbound neutrons exist. Figure 9 gives a graphical representation of the state of matter in the crust. The results shown employ the EoS of Ruester, Hempel, and Schaffner-Bielich (2006) for the outer crust, where experimentally measured binding energies have been used in combination with nuclear structure calculations with the SLy4 EDF. For the EoS of the inner crust and the core, the results of Douchin and Haensel (2001) are taken, which are based on Thomas-Fermi calculations using the same SLy4 EDF. To obtain the radial structure of the assumed NS with a mass of $1.44 M_{\odot}$, the TOV equations (36) were solved.

The outer crust is composed of completely ionized nuclei in a sea of electrons of almost constant density due to the large incompressibility of the highly degenerate electron fluid. In the standard picture, only a single nuclear species exists at a given density. These nuclei form a bcc lattice of ions as demonstrated in classical one-component plasma simulations (see Sec. III.C.6). The individual nuclei at their lattice sites can be identified as the blue dots in Fig. 9. At densities of about $10^{7} \mathrm{~g} / \mathrm{cm}^{3}$ and below, a crystal of ${ }^{56} \mathrm{Fe}$ nuclei is expected to form. With increasing density the lattice constant decreases and the electron chemical potential rises substantially. It becomes energetically favorable to squeeze electrons into the nuclei, converting protons to neutrons. A sequence of bcc lattices with more and more neutron-rich ions on the lattice sites appears the deeper one penetrates into the NS. Each change from one to the next nuclear species is connected to a 
phase transition with a jump in the density (cf. Sec. III.D.2). The series of nuclei in the outer crust is determined by their masses and is influenced strongly by shell effects. With the recent progress to measure masses of very neutron-rich nuclei experimentally with high precision, the order of ions in the outer crust from ${ }^{56} \mathrm{Fe}$ via ${ }^{62} \mathrm{Ni},{ }^{64} \mathrm{Ni},{ }^{66} \mathrm{Ni},{ }^{86} \mathrm{Kr},{ }^{84} \mathrm{Se}$, and ${ }^{82} \mathrm{Ge}$ could be established with increasing depth (Kreim et al., 2013; Wolf et al., 2013). For higher densities, the nuclear masses from theoretical models, e.g., liquid-drop or EDF type, have been used to determine the chemical composition of the outer crust. Since the early works of Salpeter (1961) and Baym, Pethick, and Sutherland (1971) the theoretical description of the outer crust is well settled and the main changes result from improvements in the theoretical description of exotic nuclei not studied experimentally so far (Haensel, Zdunik, and Dobaczewski, 1989; Haensel and Zdunik, 1990; Haensel and Pichon, 1994; Ruester, Hempel, and Schaffner-Bielich, 2006; Pearson, Goriely, and Chamel, 2011; Roca-Maza et al., 2012; Wolf et al., 2013).

At a mass density of approximately $10^{11} \mathrm{~g} / \mathrm{cm}^{3}$ the neutron drip density is reached, i.e., the neutron chemical potential becomes too high for nuclei at the lattice sites to bind additional neutrons. In Fig. 9, the contribution of these unbound neutrons, indicated by the blue color, becomes visible only at sufficiently high densities. These unbound neutrons can propagate more or less freely through the lattice, although their interaction with the lattice could modify the crystalline structure (Kobyakov and Pethick, 2014). A proper treatment of the periodic crystal structure and its effect on neutron and electron properties requires a description using band structure models as in solid state physics (Pethick and Thorsson, 1997; Chamel, 2005). Since the temperature is very low, effects of neutron pairing could be important. This is less relevant for the basic thermodynamic properties of the crust matter itself but it has to be considered for dynamic processes and thermal properties, in particular, neutron star cooling. In view of neutron superfluidity, the so-called entrainment effect has to be taken into account in hydrodynamic descriptions of the NS's inner crust and core. In this case the momentum of one fluid is not aligned with its particle current, but depends on the particle currents of all other fluids (Carter, Chamel, and Haensel, 2005a, 2005b, 2006; Chamel, 2005; Gusakov and Haensel, 2005; Chamel and Haensel, 2006; Gusakov, Kantor, and Haensel, 2009b).

The exact location of the neutron drip density is sensitive to details of the theoretical model, in particular, to the isospin dependence of the effective interaction due to the very large neutron excess encountered in the crust; see, e.g., Douchin and Haensel (2000, 2001), Steiner (2008), and Ducoin et al. (2011). The properties of nuclei also change inside matter at high densities, mostly in the inner crust of the NS when they are surrounded by a gas of neutrons and the electric field is screened by the electrons.

Several studies have been devoted to the description of nuclei in a dense medium and the effects on the EoS; see, e.g., Baym, Bethe, and Pethick (1971), Barkat, Buchler, and Ingber (1972), Ravenhall, Bennett, and Pethick (1972), Negele and Vautherin (1973), Lamb et al. (1978), Cheng, Yao, and Dai (1997), Baiko and Haensel (1999), Douchin and
Haensel (2001), Matsuzaki (2006), Ducoin et al. (2008), Papakonstantinou et al. (2013), Aymard, Gulminelli, and Margueron (2014), and Raduta, Aymard, and Gulminelli (2014). In most cases, the Wigner-Seitz approximation in spherical cells surrounding a single nucleus is employed (see Sec. III.C.6). With increasing depth inside the crust, nuclei approach each other and the action of the short-range nuclear interaction beyond the size of an individual nucleus has to be considered. First, a strong deformation of nuclei and a change in the shell structure is observed in model calculations (Oyamatsu, 1993, 1994; Douchin, Haensel, and Meyer, 2000), such that they finally touch and the sequence of classical pasta phases is found (Buchler and Barkat, 1971; Ravenhall, Pethick, and Wilson, 1983; Watanabe et al., 2003b, 2009; Maruyama et al., 2005; Avancini et al., 2009; Newton and Stone, 2009). The picture of the classical pasta phases with their specific geometries and phase transitions changes if more general shapes are allowed in full three-dimensional calculations with less restrictions on the symmetries (Watanabe et al., 2005; Nakazato, Oyamatsu, and Yamada, 2009; Okamoto et al., 2012; Schneider et al., 2014; Schuetrumpf et al., 2015). The extension of the inner crust, the types of pasta phases, and the transition density to uniform matter depends crucially on the density dependence of the symmetry energy (Pethick, Ravenhall, and Lorenz, 1995; Oyamatsu and Iida, 2007; Roca-Maza and Piekarewicz, 2008; Kubis, Porebska, and Alvarez-Castillo, 2010; Grill, Providência, and Avancini, 2012; Grill et al., 2014). The pasta phase could be relevant for the neutrino transport in the PNS and the subsequent cooling of the NS. For example, Horowitz et al. (2015) showed that the pasta phase can reduce the electrical and the thermal conductivities. The reduced electrical conductivity might be related to the observed upper limit of X-ray pulsar spin periods (Pons, Viganò, and Rea, 2013).

The crust has a subdominant effect on global properties of NSs such as mass or radius. Therefore, a crust EoS is matched often to an EoS of uniform matter from an independent model calculation. Considerable effort is required in developing an EoS which describes matter from the surface to the center of the NS in a unified manner, i.e., on the basis of the same interaction model, including a description of inhomogeneous matter in the crust. This is important for detailed predictions of NS radii and for dynamical properties. Only few such unified NS EoSs exist; see, e.g., Douchin and Haensel (2001), Fantina et al. (2013), Miyatsu, Yamamuro, and Nakazato (2013), Baldo et al. (2014), Gulminelli and Raduta (2015), and Sharma et al. (2015). Unified NS EoSs can also be obtained from the general purpose EoS discussed in Sec. V.D by applying zero (or negligibly small) temperature and $\beta$-equilibrium conditions. However, the aforementioned dedicated unified NS EoS models often give a more detailed description of nonuniform NS matter. A comparison of these classes of models is useful to investigate limitations of general purpose EoSs regarding their description of nuclei in dense and cold matter.

In contrast to a conventional star in a hadronic model, the EoS is very different for strange stars (Alcock, Farhi, and Olinto, 1986; Haensel, Zdunik, and Schaeffer, 1986), and the structure of the crust is still a matter of debate (Alford et al., 
2006; Jaikumar, Reddy, and Steiner, 2006; Oertel and Urban, 2008).

\section{Composition of the neutron star core}

The composition of matter at suprasaturation densities reached in the NS core is uncertain and, in particular, particles other than nucleons and electrons are expected to appear. In the literature muons, pions, kaons and their condensates, hyperons, nuclear resonances, and quarks have been considered (Glendenning, 1997). There is even the possibility of absolutely stable strange quark matter (Farhi and Jaffe, 1984; Witten, 1984) and pure strange stars (Alcock, Farhi, and Olinto, 1986; Haensel, Zdunik, and Schaeffer, 1986); see also Itoh (1970). In this context, the recent discovery of two NSs with masses of about $2 M_{\odot}$ (Demorest et al., 2010; Antoniadis et al., 2013; Fonseca et al., 2016) has triggered intensive discussions, since without an interaction, any additional degree of freedom softens the EoS simply by lowering the Fermi energies of the particles present. As a consequence, a lower maximum mass is obtained and many older models containing additional particles are in contradiction with the NS mass constraint.

Phenomenological quark models can easily be supplemented with the necessary repulsion at high densities. As an example, for the NJL model Lagrangian of Eq. (27) this can be achieved by adding a vector interaction term of the form

$$
\mathcal{L}_{V}=G_{V}\left(\bar{\psi} \gamma^{\mu} \psi\right)\left(\bar{\psi} \gamma_{\mu} \psi\right)
$$

Maximum NS masses above $2 M_{\odot}$ can then be obtained; see, e.g., Alford et al. (2007), Klähn et al. (2007), Weissenborn et al. (2011), Zdunik and Haensel (2013), and Buballa et al. (2014). Masuda, Hatsuda, and Takatsuka (2013) proposed that the transition from hadronic to quark matter might be a crossover potentially leading to an increase of the maximum mass. However, this scenario requires an ad hoc interpolation scheme to connect the hadronic and the quark phase (Kojo et al., 2015). The same scenario was recently applied in the context of PNSs by Masuda, Hatsuda, and Takatsuka (2016).

Hyperonic degrees of freedom are more difficult to reconcile with a $2 M_{\odot}$ NS. Most models that include hyperons predict that they appear at $n_{B} \sim(2-3) n_{\text {sat }}$ but lead at the same time to maximum NS masses of $\sim 1.4 M_{\odot}$, well below the highest observed ones. Sometimes this is called the "hyperon puzzle" in the literature (Lonardoni et al., 2015). A similar effect is observed with nuclear resonances (Drago et al., 2014) and meson condensates. It is thus obvious that additional repulsion is needed to stiffen the high-density EoS.

Different solutions have been proposed to overcome this problem. The first one is that a transition to quark matter appears at sufficiently low densities such that hyperons or other additional hadronic particles have not yet softened the EoS too much. A two family scenario with low mass compact hadronic stars and high mass quark stars was recently discussed by Drago, Lavagno, and Pagliara (2014).

Another possibility is to modify the interactions at high densities. Hyperonic interactions have been extensively studied in this respect. Since experimental data are scarce and furnish only weak constraints on the interactions at subsaturation densities, even less is known about the hyperon-nucleon $(Y N)$ and hyperon-hyperon $(Y Y)$ interactions at the relevant densities in the core of NSs (see Sec. III.A.1). Presently several phenomenological EoS models exist that contain hyperons and predict maximum NS masses in agreement with observations; see, e.g., Hofmann, Keil, and Lenske (2001a), Rikovska-Stone et al. (2007), Bednarek et al. (2012), Bonanno and Sedrakian (2012), Weissenborn, Chatterjee, and Schaffner-Bielich (2012a, 2012b), Colucci and Sedrakian (2013), Banik, Hempel, and Bandyopadhyay (2014), Lopes and Menezes (2014), van Dalen, Colucci, and Sedrakian (2014), Gomes et al. (2015), and Oertel et al. (2015). The crucial point is that the interaction is adjusted to provide the necessary repulsion.

In microscopic models the missing repulsion for hyperons is more difficult to obtain. Naturally, one would expect it to arise from three-body forces. But, using a microscopic model based on the BHF approach, Vidaña et al. (2011) found that even adding a phenomenological three-body force was not enough to allow for the existence of stars that are massive enough to be compatible with observations. Recent relativistic DBHF calculations (Katayama and Saito, 2014), including automatically part of the three-body forces, reproduce hyperonic NSs with two solar masses, but with a nuclear EoS that is either too stiff or does not give enough binding in contradiction with known properties of symmetric nuclear matter at saturation. On the other hand, in recent calculations using an auxiliary field diffusion Monte Carlo method (AFQMC) (Lonardoni, Gandolfi, and Pederiva, 2013; Lonardoni, Pederiva, and Gandolfi, 2014; Lonardoni et al., 2015), it was found that a sufficiently strong repulsive threebody force, constrained by the systematics of separation energies in a series of hypernuclei, can produce an EoS stiff enough to satisfy the $2 M_{\odot}$ constraint, even if a strong model dependence due to the phenomenological nature of the hyperonic two- and three-body forces is apparent. In conclusion, there are still many open questions regarding the role of hyperons and other additional non-nucleonic degrees of freedom in NSs.

\section{B. EoS of uniform matter at finite temperature}

The thermal properties of nuclear matter are an important subject on their own and many studies are actually performed without an astrophysical application. Examples are the $a b$ initio calculations of neutron matter at finite temperatures, some of which have been mentioned in Sec. IV.B, or studies of the nuclear liquid-gas phase transition which occurs at subsaturation densities if Coulomb and finite-size effects are neglected. The liquid-gas phase transition is an important aspect of the low-density nuclear matter EoS and has been studied extensively in the literature; see, e.g., Barranco and Buchler (1980) and Müller and Serot (1995). An example is shown in Fig. 4. In addition to BHF (Baldo and Ferreira, 1999; Baldo, Ferreira, and Nicotra, 2004) and DBHF (Ter Haar and Malfliet, 1987; Huber, Weber, and Weigel, 1998) calculations extended to finite temperature, consistent SCGF calculations have been reported by Rios et al. (2008) and Fiorilla, Kaiser, and Weise (2012). Wellenhofer et al. (2014) and Wellenhofer, 
Holt, and Kaiser (2015) studied the phase diagram of nuclear matter applying in-medium $\chi$ EFT.

Here we are mainly interested in EoSs relevant for astrophysical applications. Finite-temperature effects are of particular relevance for PNSs, CCSNe, and NS mergers and can be studied in HICs too. Although more scarce than NS EoSs, there is a variety of works considering EoSs of homogeneous matter at fixed entropies or temperatures and hadronic charge fractions. Many of these EoSs have actually been developed for studying PNSs by considering characteristic hydrostatic configurations that represent different evolutionary stages. The history concerning additional particles, such as hyperons, mesons, or quarks, is almost as long as for cold neutron stars, reaching from the discussion of quark matter formation or meson condensates to hyperons; see Prakash et al. (1997) for an early review and Pons et al. (2000), Pons, Miralles et al. (2001), Bombaci et al. (2007), Menezes and Providência (2007), Dexheimer and Schramm (2008), Yasutake and Kashiwa (2009), Beisitzer, Stiele, and Schaffner-Bielich (2014), and Masuda, Hatsuda, and Takatsuka (2016) for a few examples.

Often PNSs are approximated as isentropic, i.e., having a constant entropy per baryon $s$, and/or a fixed lepton or electron fraction $\left(Y_{L^{(e)}}\right.$ or $Y_{e}$, respectively) with or without trapped neutrinos. Sometimes isothermal PNS are considered too. If the crust and envelope of the PNS are neglected, the situation is still similar to that for cold NS: Matter is uniform and for each parameter combination of $s$ or $T$ and $Y_{L^{(e)}}$ or $Y_{e}$ the EoS is still one dimensional, i.e., it depends only on one state variable, e.g., baryon number density.

Most ab initio calculations of the EoS of nuclear matter at finite temperature concern pure neutron matter (see Sec. IV.B), or symmetric nuclear matter, since general asymmetric nuclear matter asks for more involved computations. Some exceptions exist, e.g., the EoS of Togashi and Takano (2013) uses the variational method, starting from a nuclear Hamiltonian that is composed of the Argonne v18 and Urbana IX potentials. They aim at providing a full general purpose EoS that can be applied in astrophysical simulations (see Sec. V.D). However, in their first work, they considered only uniform nuclear matter, but for various temperatures and asymmetries. To simplify computations, the frozen-correlation approximation is employed: the self-energies and correlation matrix elements are evaluated at zero temperature. This approximation is motivated by the results of Baldo and Ferreira (1999). Togashi and Takano (2013) validated it by comparing with results for fully minimized calculations.

The frozen-correlation approximation is standard in finitetemperature BHF calculations aimed to model the PNS EoS too; see, e.g., Nicotra et al. (2006a, 2006b), Burgio, Schulze, and Li (2011), and Chen et al. (2012). Effects of hyperons and/or quarks were considered too within these works relying on different models for the interactions. For instance, for the quark phase Chen et al. (2012) used a model based on the Dyson-Schwinger equations of QCD (cf. Sec. III.B.1.h), whereas Nicotra et al. (2006b) applied the MIT bag model. As known for BHF calculations (see Sec. V.A.2), the maximum mass of a cold NS including additional degrees of freedom such as hyperons or quarks lies in general well below the canonical value of $2 M_{\odot}$. Exceptions are the hybrid NS and PNS models of Chen et al. (2012) where much higher maximum masses in the vicinity of $2 M_{\odot}$ were found.

Applying phenomenological interactions to finitetemperature matter, an obvious question is whether the effective couplings, determined via zero temperature properties of strongly interacting (mainly nuclear) systems, depend on temperature. This was addressed by Fedoseew and Lenske (2015), where the thermal properties of asymmetric nuclear matter have been analyzed within a relativistic approach. The parameters of a density-dependent relativistic hadron nuclear field theory, similar to a density-dependent RMF model, have been adjusted to reproduce DBHF results for in-medium self-energies. In particular, it was shown that the temperature modifications of the nucleon-meson couplings is almost negligible. A comparison of the free energy of nuclear matter using the Brussels Skyrme interaction with the results of Fiorilla, Kaiser, and Weise (2012) leads to the same conclusion (Fantina, 2015). In Moustakidis and Panos (2009), where a momentum-dependent finite-range term is added to a Skyrme-type interaction, temperature-dependent couplings are obtained but this dependence is weak up to temperatures of $30 \mathrm{MeV}$.

Constantinou et al. (2014) thoroughly investigated the finite-temperature properties of the bulk EoS. They employed the potential model of Akmal, Pandharipande, and Ravenhall (1998), which is fitted to results from variational calculations, and compared it with the typical Skyrme EDF SKa from Köhler (1976). The latter parametrization is also applied in the H\&W EoS (see Sec. V.D.1.a). Analytical formulas are derived for all thermodynamic state variables and their derivatives at finite temperatures, simplifying the use in astrophysical applications. A similar study of the thermal properties of the EoS but for finite-range interactions was recently published by Constantinou et al. (2015).

\section{EoS of clustered matter at finite temperatures}

Complementary to investigations of bulk properties of warm and dense uniform matter, there are many works studying inhomogeneous warm matter within NSE based models (see Sec. III.C.1). Most of them do not cover the full parameter space relevant for simulations of CCSNe or NS mergers, partly since they are designed for a particular application, e.g., multifragmentation experiments or nucleosynthesis aspects. A typical problem is the omission of interactions and/or medium modifications of nuclei. As a result, the EoS does not provide a realistic description at high densities. Nevertheless, these models allow one to investigate important aspects of the EoS of clustered matter, e.g., the chemical composition and the role of excited states. The impact of the different model ingredients depends on the thermodynamic conditions.

The chemical composition of matter at baryon densities roughly below $10^{-3} \mathrm{fm}^{-3}$ and a few $\mathrm{MeV}$ temperature is mainly driven by the nuclear binding energies and the treatment of thermal excitations. Simple mass formulas provide binding energies for the widest possible range of nuclei that are considered in statistical models. A liquid-drop-type mass formula is used in the statistical model for supernova matter 
(SMSM) (Botvina and Mishustin, 2004, 2010; Buyukcizmeci, Botvina, and Mishustin, 2014). It is based on the SMM (Bondorf et al., 1995; Sagun et al., 2014) which has proven successful in the analysis of fragment yields in low-energy HICs. The parameters of the used liquid-drop mass formula, including temperature effects, have been calibrated by the analysis of experimental multifragmentation data. A liquiddrop parametrization is also used in the statistical model of Raduta and Gulminelli $(2009,2010)$. The NSE model of Blinnikov et al. (2011) considered up to 20000 nuclei, whose binding energies are taken from the theoretical mass formula of Koura et al. (2005). Binding energies from Myers and Swiatecki $(1990,1994)$ are adopted in the work of Ishizuka, Ohnishi, and Sumiyoshi (2003) incorporating about 9000 different nuclei. Results of the microscopic-macroscopic finite-range droplet model (Möller et al., 1995) or the Duflo-Zuker model (Duflo and Zuker, 1995) have been used in Gulminelli and Raduta (2015). Tables with theoretical masses from fully microscopic models, mainly EDFs, usually cover a smaller range of nuclei. Experimental binding energies (Audi, Wapstra, and Thibault, 2003; Wang et al., 2012) are available for an even smaller number of nuclei rather close to the valley of stability. They are used as far as available in some NSE based models, but have to be supplemented by theoretical masses for more exotic nuclei. The choice of different sources can result in artificial jumps of the isotopic abundances at the boundaries; see, e.g., Buyukcizmeci et al. (2013).

As an illustration, we compare in Fig. 10 nuclear abundances for typical conditions in the collapse phase of a CCSN for three statistical models. The different predictions mainly reflect the extrapolation of binding energies to neutron-rich nuclei. This depends on the choice of the mass model since the most abundant nuclei are situated outside the region where masses are experimentally known. A monomodal or bimodal structure is obtained, peaked around magic numbers in Figs. 10(b) and 10(c), demonstrating the importance of shell effects, which are missing in models based on simple mass formulas (Raduta and Gulminelli, 2010; Buyukcizmeci, Botvina, and Mishustin, 2014), as in Fig. 10(a) or models that use the Thomas-Fermi approximation for the description of nuclei, as, e.g., in the model of Aymard, Gulminelli, and Margueron (2014). Broad and even bimodal distributions cannot be represented within the SNA (see Sec. III.C.2), which is employed, e.g., in the general purpose models STOS or LS220, discussed in Sec. V.D. Nevertheless, the predictions for the average heavy nucleus show only a moderate deviation compared to that of the statistical HS(DD2) model and global thermodynamic quantities are only slightly modified (Burrows and Lattimer, 1984). However, these differences in the composition are relevant for electron-capture reactions during the collapse phase and thus can influence the dynamics of a CCSN as discussed in Sec. VI.B.1. For very neutronrich conditions, the range of nuclei considered in the table, indicated by the gray regions in Fig. 10, also matters. Different rules for determining the boundary are employed, e.g., vanishing neutron separation energies or binding energies.

With increasing temperature, excited states of nuclei are populated. These are considered explicitly in models that use temperature-dependent degeneracy factors. Usually, level densities of a Fermi-gas type are employed, e.g., those of
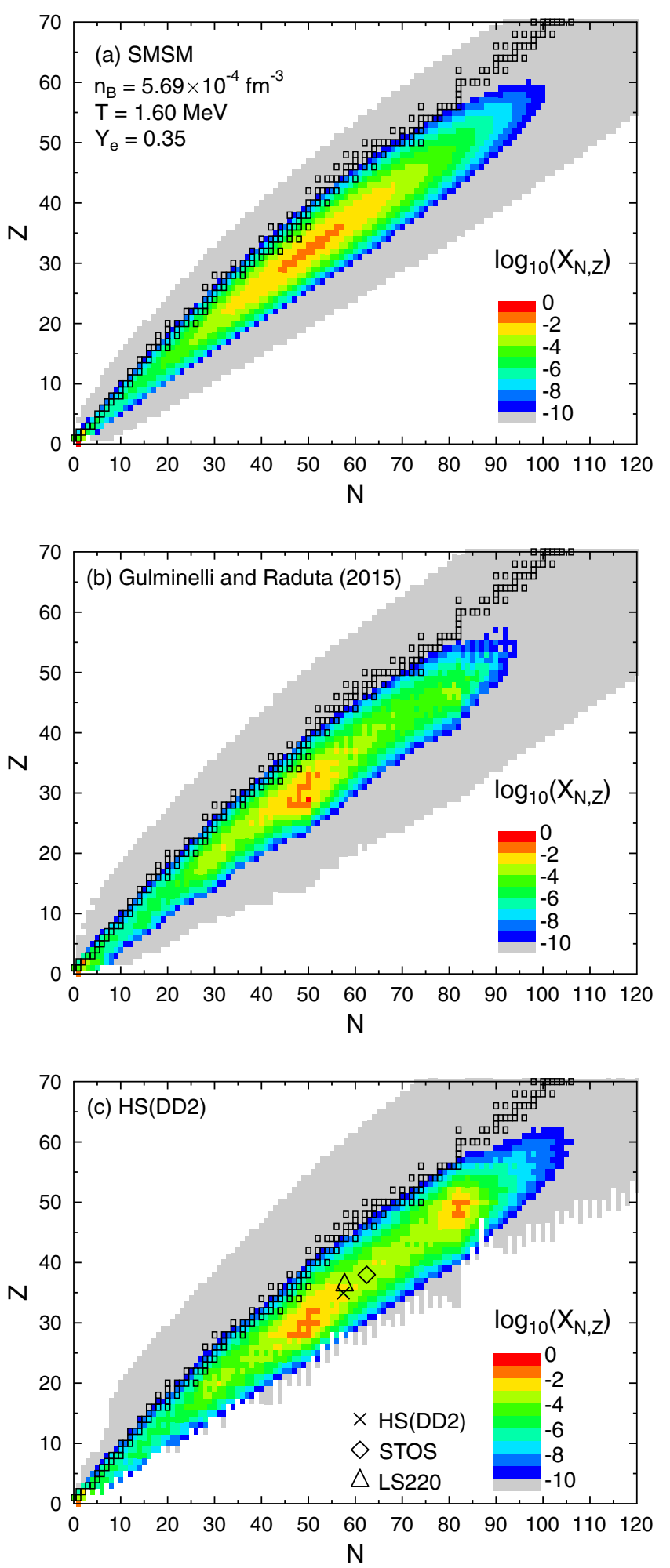

FIG. 10. Composition of matter in the center of a CCSN 6 ms before bounce at thermodynamic conditions taken from a simulation of Perego et al. (2015). The color map shows the distribution of nuclei (mass fractions) in the (a) SMSM (Buyukcizmeci, Botvina, and Mishustin, 2014), the (b) EoS of Gulminelli and Raduta (2015), and the (c) HS(DD2) model. (c) The black cross indicates the average heavy nucleus. The black diamond and triangle show the representative heavy nucleus of STOS and LS220, respectively, calculated within the SNA. 
Iljinov et al. (1992) in Raduta and Gulminelli $(2009,2010)$ or Fái and Randrup (1982) in Ishizuka, Ohnishi, and Sumiyoshi (2003) (see Sec. III.C.1.b). An alternative approach is to incorporate temperature effects directly in the mass model, e.g., in the SMSM, by introducing a temperature dependence in the coefficients of the mass formula, in particular, in bulk and surface contributions to the energy. Part of the differences in Fig. 10 also result from the treatment of excited states. The actual treatment in the model also influences the dissolution of heavy clusters with increasing temperature and the change of the abundance distributions. They are dominated more strongly by light clusters at high $T$, which are usually handled independently of the heavier nuclei using experimental binding energies and sometimes correct quantum statistics. At very low densities, the finite-temperature EoS is given model independently by the VEoS (see Sec. III.C.3). The VEoS with light species ( $p, n, d, t,{ }^{3} \mathrm{He}$, and $\alpha$ ) was discussed by Pratt, Siemens, and Usmani (1987) for conditions in HICs. Horowitz and Schwenk (2006a) considered neutrons, protons, and $\alpha$ particles as basic constituents and experimental information on binding energies and phase shifts was used in the calculation of the second virial coefficients. O'Connor et al. (2007) added ${ }^{3} \mathrm{H}$ and ${ }^{3} \mathrm{He}$ nuclei, and even heavier species were included by Mallik et al. (2008). A relativistic VEoS was already given by Venugopalan and Prakash (1992) for an interacting gas of nucleons, pions, and kaons. A general finding, present in all models, is that deuterons, tritons, and helions appear abundantly for typical conditions of supernova matter in addition to $\alpha$ particles (Sumiyoshi and Röpke, 2008; Heckel, Schneider, and Sedrakian, 2009; Typel et al., 2010; Hempel et al., 2015; Pais, Chiacchiera, and Providência, 2015). The presence of light clusters can modify weak interaction rates and therefore the dynamics of astrophysical processes (see Sec. VI).

With increasing baryon density, above approximately $10^{-3} \mathrm{fm}^{-3}$, interaction effects start to play a role and medium effects on the binding energies have to be incorporated in the statistical models. They modify the chemical composition in comparison to results of pure NSE models that use vacuum binding energies. In essentially all models, the screening of the Coulomb potential due to the electron component is taken into account in the Wigner-Seitz approximation (see Sec. III.D.2). The repulsion of nuclei is often modeled by an excluded-volume mechanism (see Sec. III.C.1.e), e.g., in Raduta and Gulminelli (2009) and Sagun et al. (2014). Raduta and Gulminelli (2010) solved the cluster partition sums by using Metropolis Monte Carlo techniques which allow one to consider configuration dependent excludedvolume corrections. A comparison of predictions from the geometric excluded-volume approach with those of the more microscopically inspired approach using mass shifts (see Sec. III.C.4) was presented by Hempel et al. (2011) concentrating on light nuclei. The dissolution of clusters with increasing density cannot be described properly in basic statistical models unless an excluded-volume mechanism or mass shifts are considered. The treatment of the homogeneous nucleonic matter contribution is relatively similar in most NSE based EoS models, employing, if at all, phenomenological mean-field approaches with various interactions; see
Sec. V.D.3 for a discussion of the compatibility with present day constraints. Interactions of unbound nucleons are not included in the SMSM, therefore it is applicable only to dilute matter. A comparison of different NSE-type models can be found in Ishizuka, Ohnishi, and Sumiyoshi (2003) and Buyukcizmeci et al. (2013) with detailed and comprehensive analyses of the nuclear composition.

\section{General purpose equations of state}

In this section we describe EoSs which cover the full thermodynamic parameter range necessary for astrophysical simulations of CCSNe or NS mergers. Such EoSs not only have to be available for finite temperatures and different charge fractions, but should include a description of nonuniform matter at subsaturation densities, where nuclei appear, and a description of homogeneous matter at high densities and/or temperatures.

There exist only a few such EoSs. To give an overview, we summarize their particle content, disregarding leptonic degrees of freedom here and in the following, and some key properties for cold NSs of the different models in Table III. We indicate if the EoS is publicly available in tabulated form or as a computer code (see Appendix A.1 for a list of different online resources). Key nuclear matter properties of the nuclear interaction models, which are used in the EoS models of Table III, are given in Table IV.

We remark that all of the presently available general purpose EoSs are included in the discussion, even though many of them are in strong disagreement with some astrophysical, experimental, or theoretical constraints. However, several of them are still used for reference applications. Any "benchmarking" of EoSs depends on which constraints are chosen from the many available in the literature, and there is not a single model that fulfills all of them, not even the very limited set of constraints that we consider later. A general purpose EoS with particular deficits can be interesting because it very much depends on the astrophysical context and the specific application whether a constraint is relevant or not. For example, cluster formation at low densities seems to be more important than the neutron matter EoS for the dynamics of CCSNe (see Sec. VI.B.1) but in NS mergers probably the opposite is the case. Because of the limited number of general purpose EoSs and their importance for astrophysical applications, we first give a complete overview. A critical discussion follows at the end in Sec. V.D.3.

\section{Nucleons and nuclei as degrees of freedom}

\section{a. $H \& W$}

The EoS of Hillebrandt, Nomoto, and Wolff (1984) and Hillebrandt and Wolff (1985) (H\&W) is one of the first EoSs that was suitable for CCSNe simulations and which is still in use today (Janka, 2012a). At low densities, a NSE model based on the work of El Eid and Hillebrandt (1980) is applied including 470 different nuclei: in addition to neutrons, protons, and $\alpha$ particles, about 450 isotopes with charge numbers $Z$ between 10 and 32 and neutron numbers $N$ ranging from stability to neutron drip as well as 20 heavier nuclei from the $\mathrm{Zr}$ and $\mathrm{Pb}$ region are included. To account for excited 
TABLE III. Characteristic properties of the currently existing general purpose EoSs. Top part: EoSs containing nucleons and nuclei; bottom part: EoSs including additional hadronic or quark degrees of freedom. Listed are the nuclear interaction model used, the included particle degrees of freedom, the maximum mass $M_{\max }$ of cold, spherical (nonrotating) NSs, and their radii at a fiducial gravitational mass $M_{G}$ of $1.4 M_{\odot}$. We included in addition the compactness $\Xi=G M_{G} / R$ of the maximum mass configuration. Empty entries indicate that the value was not available to the authors. In nuclear interactions labeled with ${ }^{*}$, the nucleon masses have been changed to experimental values without a refitting of the coupling constants. This induces a marginal change of the interaction.

\begin{tabular}{|c|c|c|c|c|c|c|c|}
\hline Model & $\begin{array}{l}\text { Nuclear } \\
\text { interaction }\end{array}$ & $\begin{array}{l}\text { Degrees of } \\
\text { freedom }\end{array}$ & $\begin{array}{l}M_{\max }, \\
\left(M_{\odot}\right)\end{array}$ & $\begin{array}{l}R_{1.4 M_{\odot}}, \\
\quad(\mathrm{km})\end{array}$ & $\Xi$ & $\begin{array}{l}\text { Publicly } \\
\text { available }\end{array}$ & References \\
\hline $\mathrm{H} \& \mathrm{~W}$ & SKa & $n, p, \alpha,\left\{\left(A_{i}, Z_{i}\right)\right\}$ & $2.21^{\mathrm{a}}$ & $13.9^{\mathrm{a}}$ & & No & $\begin{array}{l}\text { El Eid and Hillebrandt (1980) } \\
\text { and Hillebrandt, Nomoto, } \\
\text { and Wolff (1984) }\end{array}$ \\
\hline LS180 & LS180 & $n, p, \alpha,(A, Z)$ & 1.84 & 12.2 & 0.27 & Yes & Lattimer and Swesty (1991) \\
\hline LS220 & LS220 & $n, p, \alpha,(A, Z)$ & 2.06 & 12.7 & 0.28 & Yes & Lattimer and Swesty (1991) \\
\hline LS375 & LS375 & $n, p, \alpha,(A, Z)$ & 2.72 & 14.5 & 0.32 & Yes & Lattimer and Swesty (1991) \\
\hline STOS & TM1 & $n, p, \alpha,(A, Z)$ & 2.23 & 14.5 & 0.26 & Yes & $\begin{array}{l}\text { Shen et al. (1998a, 1998b, } \\
\text { 2011) }\end{array}$ \\
\hline FYSS & TM1 & $n, p, d, t, h, \alpha,\left\{\left(A_{i}, Z_{i}\right)\right\}$ & 2.22 & 14.4 & 0.26 & No & $\begin{array}{l}\text { Furusawa, Sumiyoshi et al. } \\
\text { (2013) }\end{array}$ \\
\hline HS(TM1) & $\mathrm{TM} 1 *$ & $n, p, d, t, h, \alpha,\left\{\left(A_{i}, Z_{i}\right)\right\}$ & 2.21 & 14.5 & 0.26 & Yes & $\begin{array}{l}\text { Hempel and Schaffner-Bielich } \\
\text { (2010) and Hempel et al. } \\
\text { (2012) }\end{array}$ \\
\hline HS(TMA) & TMA* & $n, p, d, t, h, \alpha,\left\{\left(A_{i}, Z_{i}\right)\right\}$ & 2.02 & 13.9 & 0.25 & Yes & $\begin{array}{l}\text { Hempel and Schaffner-Bielich } \\
\text { (2010) }\end{array}$ \\
\hline HS(FSU) & FSUgold* & $n, p, d, t, h, \alpha,\left\{\left(A_{i}, Z_{i}\right)\right\}$ & 1.74 & 12.6 & 0.23 & Yes & $\begin{array}{l}\text { Hempel and Schaffner-Bielich } \\
\text { (2010) and Hempel et al. } \\
\text { (2012) }\end{array}$ \\
\hline HS(NL3) & NL3* & $n, p, d, t, h, \alpha,\left\{\left(A_{i}, Z_{i}\right)\right\}$ & 2.79 & 14.8 & 0.31 & Yes & $\begin{array}{l}\text { Hempel and Schaffner-Bielich } \\
\text { (2010) and Fischer, Hempel } \\
\text { et al. (2014) }\end{array}$ \\
\hline $\mathrm{HS}(\mathrm{DD} 2)$ & DD2 & $n, p, d, t, h, \alpha,\left\{\left(A_{i}, Z_{i}\right)\right\}$ & 2.42 & 13.2 & 0.30 & Yes & $\begin{array}{l}\text { Hempel and Schaffner-Bielich } \\
\text { (2010) and Fischer, Hempel } \\
\text { et al. (2014) }\end{array}$ \\
\hline HS(IUFSU) & IUFSU* & $n, p, d, t, h, \alpha,\left\{\left(A_{i}, Z_{i}\right)\right\}$ & 1.95 & 12.7 & 0.25 & Yes & $\begin{array}{l}\text { Hempel and Schaffner-Bielich } \\
\text { (2010) and Fischer, Hempel } \\
\text { et al. (2014) }\end{array}$ \\
\hline SFHo & SFHo & $n, p, d, t, h, \alpha,\left\{\left(A_{i}, Z_{i}\right)\right\}$ & 2.06 & 11.9 & 0.30 & Yes & $\begin{array}{l}\text { Steiner, Hempel, and Fischer } \\
\text { (2013) }\end{array}$ \\
\hline SFHx & SFHx & $n, p, d, t, h, \alpha,\left\{\left(A_{i}, Z_{i}\right)\right\}$ & 2.13 & 12.0 & 0.29 & Yes & $\begin{array}{l}\text { Steiner, Hempel, and Fischer } \\
\text { (2013) }\end{array}$ \\
\hline SHT(NL3) & NL3 & $n, p, \alpha,\left\{\left(A_{i}, Z_{i}\right)\right\}$ & 2.78 & 14.9 & 0.31 & Yes & $\begin{array}{l}\text { Shen, Horowitz, and Teige } \\
\text { (2011) }\end{array}$ \\
\hline $\mathrm{SHO}(\mathrm{FSU})$ & FSUgold & $n, p, \alpha,\left\{\left(A_{i}, Z_{i}\right)\right\}$ & 1.75 & 12.8 & 0.23 & Yes & $\begin{array}{l}\text { Shen, Horowitz, and } \\
\text { O'Connor (2011) }\end{array}$ \\
\hline SHO(FSU2.1) & FSUgold2.1 & $n, p, \alpha,\left\{\left(A_{i}, Z_{i}\right)\right\}$ & 2.12 & 13.6 & 0.26 & Yes & $\begin{array}{l}\text { Shen, Horowitz, and } \\
\text { O'Connor (2011) }\end{array}$ \\
\hline $\mathrm{LS} 220 \Lambda$ & LS220 & $n, p, \alpha,(A, Z), \Lambda$ & 1.91 & 12.4 & 0.29 & Yes & $\begin{array}{l}\text { Oertel, Fantina, and Novak } \\
\text { (2012) and Gulminelli et al. } \\
\text { (2013) }\end{array}$ \\
\hline LS220 $\pi$ & LS220 & $n, p, \alpha,(A, Z), \pi$ & 1.95 & 12.2 & 0.29 & No & $\begin{array}{l}\text { Oertel, Fantina, and Novak } \\
\text { (2012) and Peres, Oertel, } \\
\text { and Novak (2013) }\end{array}$ \\
\hline $\mathrm{BHB} \Lambda$ & DD2 & $n, p, d, t, h, \alpha,\left\{\left(A_{i}, Z_{i}\right)\right\}, \Lambda$ & 1.96 & 13.2 & 0.25 & Yes & $\begin{array}{l}\text { Banik, Hempel, and } \\
\text { Bandyopadhyay (2014) }\end{array}$ \\
\hline $\mathrm{BHB} \Lambda \phi$ & DD2 & $n, p, d, t, h, \alpha,\left\{\left(A_{i}, Z_{i}\right)\right\}, \Lambda$ & 2.11 & 13.2 & 0.27 & Yes & $\begin{array}{l}\text { Banik, Hempel, and } \\
\text { Bandyopadhyay (2014) }\end{array}$ \\
\hline $\operatorname{STOS} \Lambda$ & TM1 & $n, p, \alpha,(A, Z), \Lambda$ & 1.90 & 14.4 & 0.23 & Yes & Shen et al. (2011) \\
\hline STOSYA30 & TM1 & $n, p, \alpha,(A, Z), Y$ & 1.59 & 14.6 & 0.17 & Yes & Ishizuka et al. (2008) \\
\hline STOSYA30 $\pi$ & TM1 & $n, p, \alpha,(A, Z), Y, \pi$ & 1.62 & 13.7 & 0.19 & Yes & Ishizuka et al. (2008) \\
\hline STOSY0 & TM1 & $n, p, \alpha,(A, Z), Y$ & 1.64 & 14.6 & 0.18 & Yes & Ishizuka et al. (2008) \\
\hline $\operatorname{STOSY} 0 \pi$ & TM1 & $n, p, \alpha,(A, Z), Y, \pi$ & 1.67 & 13.7 & 0.19 & Yes & Ishizuka et al. (2008) \\
\hline STOSY30 & TM1 & $n, p, \alpha,(A, Z), Y$ & 1.65 & 14.6 & 0.18 & Yes & Ishizuka et al. (2008) \\
\hline STOSY $30 \pi$ & TM1 & $n, p, \alpha,(A, Z), Y, \pi$ & 1.67 & 13.7 & 0.19 & Yes & Ishizuka et al. (2008) \\
\hline STOSY90 & TM1 & $n, p, \alpha,(A, Z), Y$ & 1.65 & 14.6 & 0.18 & Yes & Ishizuka et al. (2008) \\
\hline $\operatorname{STOSY} 90 \pi$ & TM1 & $n, p, \alpha,(A, Z), Y, \pi$ & 1.67 & 13.7 & 0.19 & Yes & Ishizuka et al. (2008) \\
\hline $\operatorname{STOS} \pi$ & TM1 & $n, p, \alpha,(A, Z), \pi$ & 2.06 & 13.6 & 0.26 & No & $\begin{array}{l}\text { Nakazato, Sumiyoshi, and } \\
\text { Yamada (2008) }\end{array}$ \\
\hline
\end{tabular}


TABLE III. (Continued)

\begin{tabular}{|c|c|c|c|c|c|c|c|}
\hline Model & $\begin{array}{l}\text { Nuclear } \\
\text { interaction }\end{array}$ & $\begin{array}{l}\text { Degrees of } \\
\text { freedom }\end{array}$ & $\begin{array}{l}M_{\max }, \\
\left(M_{\odot}\right)\end{array}$ & $\begin{array}{l}R_{1.4 M_{\odot}} \\
\quad(\mathrm{km})\end{array}$ & $\Xi$ & $\begin{array}{l}\text { Publicly } \\
\text { available }\end{array}$ & References \\
\hline $\operatorname{STOSQ} 209 \mathrm{n} \pi$ & TM1 & $n, p, \alpha,(A, Z), \pi, q$ & 1.85 & 13.6 & 0.21 & No & $\begin{array}{l}\text { Nakazato, Sumiyoshi, and } \\
\text { Yamada (2008) }\end{array}$ \\
\hline STOSQ162n & TM1 & $n, p, \alpha,(A, Z), q$ & 1.54 & & & No & $\begin{array}{l}\text { Nakazato, Sumiyoshi, and } \\
\text { Yamada (2013) }\end{array}$ \\
\hline STOSQ184n & TM1 & $n, p, \alpha,(A, Z), q$ & 1.36 & $\ldots{ }^{b}$ & & No & $\begin{array}{l}\text { Nakazato, Sumiyoshi, and } \\
\text { Yamada (2013) }\end{array}$ \\
\hline STOSQ209n & TM1 & $n, p, \alpha,(A, Z), q$ & 1.81 & 14.4 & 0.20 & No & $\begin{array}{l}\text { Nakazato, Sumiyoshi, and } \\
\text { Yamada }(2008,2013)\end{array}$ \\
\hline STOSQ139s & TM1 & $n, p, \alpha,(A, Z), q$ & 2.08 & 12.6 & 0.26 & Yes & $\begin{array}{l}\text { Sagert et al. (2012) and } \\
\quad \text { Fischer, Klähn et al. (2014) }\end{array}$ \\
\hline STOSQ145s & TM1 & $n, p, \alpha,(A, Z), q$ & 2.01 & 13.0 & 0.25 & Yes & Sagert et al. (2012) \\
\hline STOSQ155s & TM1 & $n, p, \alpha,(A, Z), q$ & 1.70 & 9.93 & 0.25 & Yes & Fischer et al. (2011) \\
\hline STOSQ162s & TM1 & $n, p, \alpha,(A, Z), q$ & 1.57 & 8.94 & 0.26 & Yes & Sagert et al. (2009) \\
\hline STOSQ165s & TM1 & $n, p, \alpha,(A, Z), q$ & 1.51 & 8.86 & 0.25 & Yes & Sagert et al. (2009) \\
\hline
\end{tabular}

${ }^{\mathrm{a}}$ Values taken from Marek and Janka (2009).

${ }^{\mathrm{b}} M_{\text {max }}$ below $1.4 M_{\odot}$.

states, nuclear level densities have been constructed based on HF potentials using the grand partition function approach (Huizenga and Moretto, 1972; Wolff, 1980). For densities above $3 \times 10^{12} \mathrm{~g} / \mathrm{cm}^{3}$, the EoS is computed in the SNA (see Sec. III.C.2), using the thermal HF method. The nuclear interaction is of the Skyrme type using the parameter set SKa (Köhler, 1976).

\section{b. $L S$}

The EoS by Lattimer and Swesty (1991) (LS) considers nucleons, $\alpha$ particles, and heavy nuclei in the SNA (see Sec. III.C.2) as degrees of freedom. The latter are described with a medium-dependent liquid-drop model. For nucleons, nonrelativistic Fermi-Dirac statistics is used, and a simplified momentum-independent nucleon-nucleon interaction is employed which results in constant effective nucleon masses equal to the applied vacuum masses. Interactions between the gas of nucleons, $\alpha$ particles, and heavy nuclei are taken into account through an excluded-volume mechanism. $\alpha$ particles are treated as hard spheres of volume $v_{\alpha}=24 \mathrm{fm}^{3}$ forming an ideal Boltzmann gas, neglecting excited states. As the density increases, nuclei undergo geometrical shape deformations, until they dissolve in favor of homogeneous nuclear matter above approximately saturation density. The formation of nonspherical nuclei and bubble phases is described by modifying the Coulomb and surface energies of nuclei. The phase transition to bulk nuclear matter is treated by a Maxwell construction between the two phases.

The LS EoS exists for three different parametrizations of the nucleonic interaction, which are usually denoted according to their value of the incompressibility $K$ of 180,220 , and $375 \mathrm{MeV}$. Nowadays the version with $K=220 \mathrm{MeV}$ is considered the most relevant of the three, since it is the best compatible with the various constraints on the EoS (see Sec. V.D.3).

\section{c. STOS}

The EoS by Shen et al. (1998a, 1998b, 2011) (STOS) is another widely used general purpose EoS. It assumes the same degrees of freedom as the LS EoS: neutrons, protons, and $\alpha$ particles as well as one heavy nucleus in the SNA. For nucleons a RMF model with nonlinear meson self-interactions is used with the parametrization TM1 (Sugahara and Toki, 1994). $\alpha$ particles are again described as an ideal MaxwellBoltzmann gas with excluded-volume corrections. Excited states of $\alpha$ particles are neglected. The properties of the representative heavy nucleus are obtained from Wigner-Seitz cell calculations within the Thomas-Fermi approximation for parametrized density distributions of nucleons and $\alpha$ particles. The translational energy and entropy contribution of heavy nuclei is not taken into account.

Zhang and Shen (2014) investigated the accuracy of the parametrized density distributions of STOS in comparison with fully self-consistent Thomas-Fermi calculations. They concluded that overall there are only small differences. In detail it was found that the free energies of the original STOS EoS are slightly too low compared to the self-consistent solutions. This and other differences were related to a too small value of the coefficient of the (surface) gradient energy of the density distribution used in STOS for the description of nuclei, which is not consistent with the employed TM1 interaction. In addition, Zhang and Shen (2014) studied the effect of a possible bubble phase for the transition to uniform nuclear matter and found that the transition can be shifted to slightly higher densities.

\section{d. FYSS}

The EoS of Furusawa et al. (2011) and Furusawa, Sumiyoshi et al. (2013) (FYSS) can be seen as an extension of the STOS EoS at subsaturation densities. The same RMF parametrization TM1 is employed for the nuclear interaction as in the STOS EoS. A distribution of various light nuclei and heavy nuclei up to $Z \sim 1000$ is included. Heavy nuclei are not described by the Thomas-Fermi approximation as in STOS but by a liquid-droptype formulation with temperature-dependent bulk energies. Shell effects are incorporated by extracting the difference of the liquid-drop binding energies compared to experimental (Audi, Wapstra, and Thibault, 2003) and theoretical values (Koura et al., 2005). A phenomenological density dependence of the shell effects is introduced, assuming that these vanish at $n_{\text {sat }}$. 
For light nuclei, it incorporates the Pauli-blocking shifts of Typel et al. (2010). Furthermore, light nuclei receive selfenergy shifts originating from the mesonic mean fields. As an additional phenomenological interaction, excluded-volume effects are applied for nucleons, light nuclei, and heavy nuclei. In addition to standard spherical nuclei, a bubble phase with low-density holes in matter of higher density is also considered. The FYSS EoS has been used to explore the effect of light nuclei in CCSN simulations (Furusawa, Nagakura et al., 2013).

\section{e. $H S$}

The basic model of the HS EoS (Hempel and SchaffnerBielich, 2010) (HS) belongs to the class of extended NSE models and describes matter as a "chemical" mixture of nuclei and unbound nucleons in NSE. Nuclei are treated as classical Maxwell-Boltzmann particles, nucleons with RMF models employing different parametrizations. Several thousands of nuclei are considered, including light ones. Binding energies are taken either from experimental measurements (Audi, Wapstra, and Thibault, 2003) or from various theoretical nuclear structure calculations (Möller et al., 1995; Lalazissis, Raman, and Ring, 1999; Geng, Toki, and Meng, 2005). The latter are chosen such that they were calculated for the same RMF parametrization as the one applied to nucleons if available; otherwise, the data from Möller et al. (1995) are used. The following medium modifications are incorporated for nuclei: screening of the Coulomb energies by the surrounding gas of electrons in the Wigner-Seitz approximation, excited states in the form of an internal partition function using the level density of Fái and Randrup (1982), which is adapted from the NSE model of Ishizuka, Ohnishi, and Sumiyoshi (2003), and excluded-volume effects. Note that further explicit medium modifications of nuclei are not considered in HS. Since the description of heavy nuclei is based on experimental nuclear masses, the HS EoS includes the correct shell effects of nuclei in vacuum. On the other hand, the use of nuclear mass tables limits the maximum mass and charge numbers of nuclei (Buyukcizmeci et al., 2013).

The first version Hempel and Schaffner-Bielich (2010) used the RMF parametrization TMA (Toki et al., 1995). A few aspects of the model have been changed in the later versions (Hempel et al., 2012), namely, a cutoff for the highest excitation energy of nuclei is introduced, experimental nucleon masses are used, and only nuclei left of the neutron drip line are considered. At present, EoS tables are available for the following RMF parametrizations: TM1 (Sugahara and Toki, 1994; Hempel et al., 2012), TMA (Toki et al., 1995; Hempel and Schaffner-Bielich, 2010; Hempel et al., 2012), FSUgold (Todd-Rutel and Piekarewicz, 2005; Hempel et al., 2012), NL3 (Lalazissis, König, and Ring, 1997; Fischer, Hempel et al., 2014), DD2 (Typel et al., 2010; Fischer, Hempel et al., 2014), and IUFSU (Fattoyev et al., 2010; Fischer, Hempel et al., 2014). We denote them by "HS(x)," where " $\mathrm{x}$ " indicates the nuclear interaction employed.

\section{f. SFHo and SFHx}

Two additional EoSs based on HS were published by Steiner, Hempel, and Fischer (2013), SFHo ("o" for optimal) and SFHx ("x" for extreme) with new RMF parametrizations that were fitted to some NS radius determinations. These two EoSs result in rather compact NSs and have moderately, respectively, very low values of the slope parameter of the symmetry energy L (Steiner, Hempel, and Fischer, 2013; Fischer, Hempel et al., 2014; Hempel et al., 2015).

\section{g. SHT(NL3), SHO(FSU), and SHO(FSU2.1)}

The EoSs of Shen, Horowitz, and O'Connor (2011) and Shen, Horowitz, and Teige (2011) are based on different underlying physical descriptions in different regimes of density and temperature. Uniform nuclear matter at high densities and temperatures is described by a RMF model. At intermediate densities, the same RMF model is used within calculations of nonuniform matter, generating a representative heavy nucleus and unbound nucleons, but no light nuclei. At low densities and temperatures, a special form of the VEoS is used which includes virial coefficients up to second order among nucleons and $\alpha$ particles. The VEoS is not using Fermi-Dirac statistics for nucleons, but only incorporates corrections for it as part of the virial coefficients. Nuclei with mass numbers $A=2$ and 3 are not considered as explicit degrees of freedom, but 8980 nuclei with mass number $A \geq 12$ are included. The contribution of heavy nuclei in NSE is modeled as a noninteracting Maxwell-Boltzmann gas without considering excluded-volume effects. Coulomb screening is included for heavy nuclei, but not for $\alpha$ particles. Note that $\alpha$ particles are present only in the virial part of the EoS, which is completely independent of the RMF interaction. The three different prescriptions are merged to a single table by minimizing the free energy. In addition, a smoothing and interpolation procedure is applied (Shen, Horowitz, and O'Connor, 2011; Shen, Horowitz, and Teige, 2011).

The EoSs of G. Shen et al. are available for two different RMF interactions: NL3 (Shen, Horowitz, and Teige, 2011) [SHT(NL3)] and FSUgold (Shen, Horowitz, and O'Connor, 2011). A density dependence of the scalar meson-nucleon coupling was introduced below $5 \times 10^{-3} \mathrm{fm}^{-3}$ in case of the NL3 interaction in order to match the energy per nucleon of a unitary neutron gas (Shen, Horowitz, and Teige, 2010). Since the FSUgold parametrization leads to a maximum NS mass of only $1.7 M_{\odot}$, an additional phenomenological pressure contribution was introduced for densities above $0.2 \mathrm{fm}^{-3}$, leading to a sufficiently high maximum mass of $2.1 M_{\odot}$. This EoS was called "FSU2.1" and we abbreviate it as SHO(FSU2.1), and the EoS with the unmodified FSUgold parametrization SHO(FSU).

\section{Including additional degrees of freedom}

An EoS covering the whole thermodynamic parameter range relevant for CCSNe and NS mergers should be able to correctly describe cold $\beta$-equilibrated NSs. As discussed in Sec. V.A.2, it might turn out that only nucleonic matter is present in cold NSs. However, this does not mean that additional degrees of freedom could not occur in stellar core-collapse events and NS mergers, where matter is strongly heated in addition to being compressed to densities above nuclear matter saturation density. The temperatures and densities reached can become so high that a traditional description in terms of electrons, nuclei, and nucleons is no longer adequate. Compared with the cold NS EoS, 
temperature effects favor the appearance of additional particles such as pions and hyperons and they become abundant in this regime. A transition to quark matter can also not be excluded. In recent years, some models have been developed which extended existing purely nuclear models (as discussed in the previous section) by including pions, hyperons, or quarks.

Let us start with the models including hyperons. Ishizuka et al. (2008) and Oertel, Fantina, and Novak (2012) considered the whole baryon octet. The former EoS is an extension of the STOS EoS by Shen et al. (1998a) and the latter of the LS220 EoS (Lattimer and Swesty, 1991). Ishizuka et al. (2008) fixed the hyperonic interactions following a standard procedure for RMF models. For the vector couplings, symmetry constraints are imposed, assuming $S U(6)$ flavor symmetry following Schaffner and Mishustin (1996) in the isoscalar sector and isospin symmetry in the isovector one. The remaining scalar couplings of hyperons to nucleons are adjusted to reproduce standard values of the single-particle hyperonic potentials in symmetric nuclear matter at saturation density, extracted from hypernuclear data (see Sec. III.A.1). These single-particle potentials are given by (see Sec. III.B.2.a)

$$
U_{j}=V_{j}-S_{j}
$$

involving scalar and vector self-energies. Standard values for $U_{\Lambda}=-30 \mathrm{MeV}$ and $U_{\Xi}=-15 \mathrm{MeV}$ are assumed, whereas the situation for $U_{\Sigma}$ is ambiguous and Ishizuka et al. (2008) presented several versions of the EoS with $U_{\Sigma}=-30,0,30$, $90 \mathrm{MeV}$ ("STOSYxxx," where "xxx" indicates the value of the potential, and a prepended " $\mathrm{A}$ " if it is attractive). In the following discussion, we keep the version with $U_{\Sigma}=+30 \mathrm{MeV}$. Oertel, Fantina, and Novak (2012) added hyperons by extending the model by Balberg and Gal (1997) to finite temperature. This model is a nonrelativistic potential model similar to the one in Lattimer and Swesty (1991) for the nuclear part. The hyperonic couplings are readjusted to remain compatible with the single-particle hyperonic potentials in nuclear matter, but, at the same time, predict maximum NS masses in approximate agreement with the measurements of Demorest et al. (2010).

Ishizuka et al. (2008) and Oertel, Fantina, and Novak (2012) presented models, in which pions are also included. The former is denoted by "STOSY $\pi \mathrm{xxx}$." Pions are treated as an ideal Bose gas. Obviously, without interactions, $\pi^{-}$will form a Bose condensate below some critical temperature, depending on the density, as discussed extensively (Migdal et al., 1990; Glendenning, 1997). It is now commonly assumed that there is an $s$-wave $\pi N$ repulsion, preventing pions from condensing. Ishizuka et al. (2008) showed that indeed, adding an effective $\pi N$ interaction, the domain in temperature and density where pions condense is strongly reduced. The main effect of pions on the EoS occurs, however, at high temperature and the ideal gas should be a good approximation in this regime. A simplified version including only pions in the LS220 EoS is used by Peres, Oertel, and Novak (2013) ("LS220 $\pi$ ”). Nakazato, Sumiyoshi, and Yamada (2008) extended the STOS EoS in the same way ("STOS $\pi$ ").
Subsequently different models including only $\Lambda$ hyperons have been developed. The first one is the work by Shen et al. (2011) ("STOS $\Lambda$ "), which is very similar to the work by Ishizuka et al. (2008), except that slightly different hyperon interactions are employed. The motivation is that the $\Lambda$ represents, together with the $\Sigma^{-}$hyperon, probably the most important hyperonic degree of freedom in hot dense supernova matter. Thus, including the $\Lambda$ allows for discussing general features of the effects coming from the hyperonic degrees of freedom without the necessity of resolving the complicated particle composition in the presence of many different hyperons. In addition, the $\Lambda N$ and $\Lambda \Lambda$ interactions are the best constrained from experimental data. And, since less degrees of freedom are populated, the NS maximum mass is less reduced from that of purely nucleonic EoSs. An extended version of the LS220 EoS including only $\Lambda$ hyperons

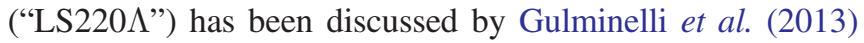
and Peres, Oertel, and Novak (2013). It has the feature that a strangeness driven first-order phase transition occurs at the onset of hyperons (Schaffner-Bielich and Gal, 2000; Schaffner-Bielich et al., 2002; Gulminelli, Raduta, and Oertel, 2012; Oertel et al., 2016) (see Sec. III.D). Banik, Hempel, and Bandyopadhyay (2014) constructed two different extended versions of the HS statistical model including $\Lambda$ hyperons. The density-dependent RMF parametrization DD2 is employed and the setup for the hyperonic couplings is similar to the one used by Ishizuka et al. (2008), i.e., assuming $S U(6)$ symmetries for the vector couplings. The value of the $\Lambda$ single-particle potential in symmetric nuclear matter determines the remaining scalar couplings. The basic model is denoted as $\mathrm{BHB} \Lambda$ and the version including additional short-range repulsion in the $\Lambda \Lambda$ channel by $\mathrm{BHB} \Lambda \phi$.

The onset density for hyperons lies between 2 and 3 times $n_{\text {sat }}$ at low temperatures. As expected, upon increasing the temperature, the density domain where hyperons appear is enlarged, in particular, above 15-20 MeV; see Fig. 11. Note that due to the presence of light nuclei in the $\mathrm{BHB} \Lambda \phi \mathrm{EoS}$ (Banik, Hempel, and Bandyopadhyay, 2014), hyperons appear at a much higher temperature in a large density domain than in the other models. Pions become more abundant at high temperatures too. This can also be seen from Fig. 12. In the bottom panels, the different particle number fractions are shown as a function of temperature for $n_{B}=0.15$ [Fig. 12(e)] and $0.3 \mathrm{fm}^{-3}$ [Fig. 12(f)] and for $Y_{q}=0.1$. The influence of $Y_{q}$ on the appearance of (neutral) $\Lambda$ hyperons is less important than for charged particles. The more asymmetric the matter, the higher is the charge chemical potential, and the higher the abundance of charged particles. In neutron-rich matter the charge chemical potential is negative, favoring negatively charged particles. This is the reason why in NS matter, $\Sigma^{-}$or $\Xi^{-}$can become enhanced with respect to $\Lambda$ hyperons, even if they have a higher mass. Thermal effects alleviate the influence of the chemical potential (Ishizuka et al., 2008; Oertel, Fantina, and Novak, 2012). If the baryon number density remains constant, the overall hyperon fractions decrease with increasing $Y_{q}$ (Prakash et al., 1997).

Concerning the influence on thermodynamic properties, pressure and energy density are shown as functions of temperature for different models in the upper and middle 

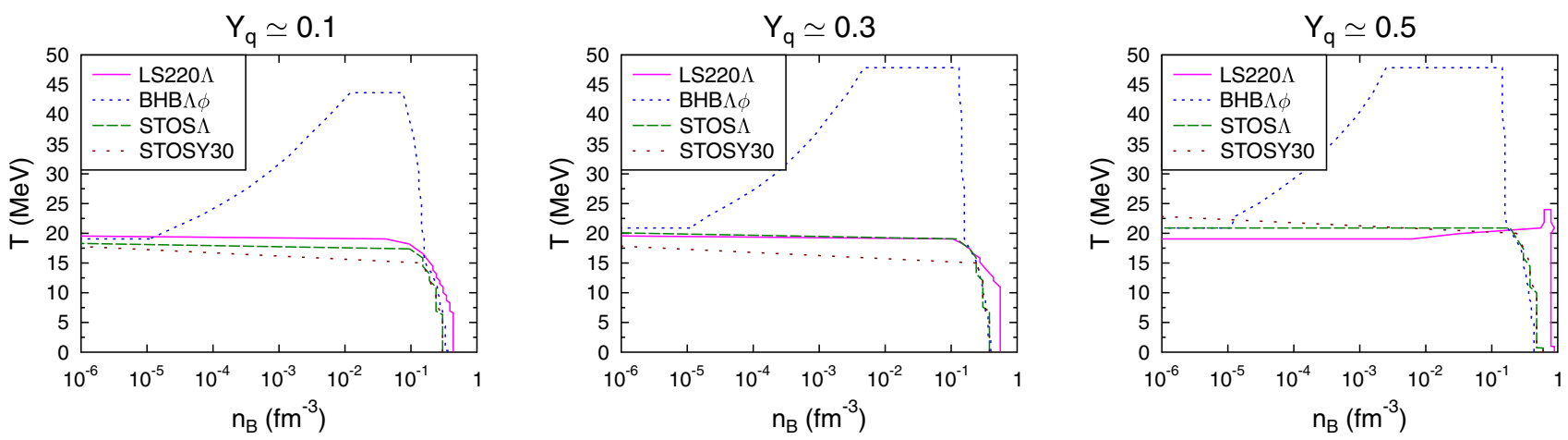

FIG. 11. The lines delimit regions in temperature and baryon number density where the number density fractions of $\Lambda$ hyperons exceed $10^{-4}$ for the different models. From left to right corresponds to a fixed hadronic charge fraction $Y_{q}=0.1,0.3$, and 0.5 . $\Lambda$ hyperons appear at high densities and temperatures, i.e., in the upper right part of each panel.

panels of Fig. 12. The STOS (Shen et al., 1998a), LS (Lattimer and Swesty, 1991), and HS(DD2) EoSs are compared with their corresponding versions including hyperons (Ishizuka et al., 2008; Shen et al., 2011; Gulminelli et al., 2013; Banik, Hempel, and Bandyopadhyay, 2014) and/or pions (Ishizuka et al., 2008; Nakazato, Sumiyoshi, and Yamada, 2008; Peres, Oertel, and Novak, 2013). As seen from Fig. 12, the effect of the additional particles on the thermodynamic quantities is not negligible for high density and temperature. The main effect is a reduction of pressure due to the additional degrees of freedom.

There exist EoSs in which the nuclear model of Shen et al. (1998a) has been supplemented with a phase transition to quark matter at high density and temperature too. In Nakazato, Sumiyoshi, and Yamada $(2008,2013)$ and the work by Sagert et al. (2009, 2012), Fischer et al. (2011), and Fischer, Klähn et al. (2014) the MIT bag model (Chodos et al., 1974; Farhi and Jaffe, 1984) is applied to the quark matter phase. The transition from hadronic matter to quark matter is described by a Gibbs construction in both cases (see Sec. III.D). In addition to the nuclear interaction, the parameters of the model are the bag constant $B$ and the strange quark mass, and possibly the coupling constant of strong interaction corrections. These parameters determine the densities for the onset of the quarkhadron phase coexistence region and the pure quark phase. It can be observed that the onset density for the mixed phase is noticeably lowered with decreasing charge fraction. In particular, this means that the critical density in asymmetric CCSN or NS matter can be lower than in symmetric matter. Furthermore, it was found that the critical density is
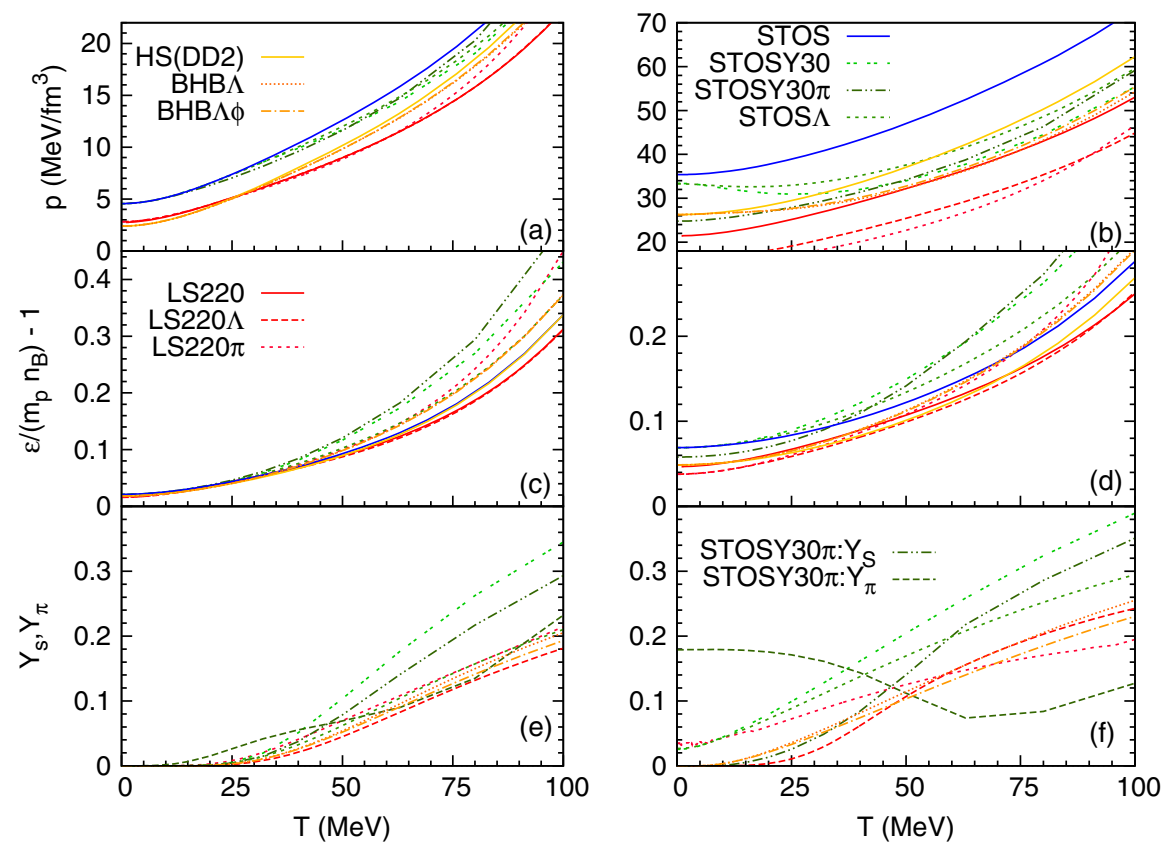

FIG. 12. Thermodynamic quantities as functions of temperature for $n_{B}=0.15$ (left) and $0.3 \mathrm{fm}^{-3}$ (right), corresponding roughly to 1 and 2 times nuclear matter saturation density, and a charge fraction of $Y_{q}=0.1$. The upper panels show the pressure, the middle ones the scaled internal energy per baryon with respect to the proton mass, and the lower ones the number fractions of additional particles. These are $\Lambda$ hyperons for "STOS $\Lambda$," "LS220 $\Lambda$," "BHB $\Lambda$," and "BHB $\Lambda \phi$ "; all hyperons for "STOSY30" and "STOSY30 $\pi^{\prime \prime}$, and the $\pi^{-}$fraction for "LS220 $\pi$." See the text for an explanation of the acronyms. 
significantly reduced due to weak equilibrium with respect to strangeness. A value close to nuclear saturation or even below is thus not in contradiction with any terrestrial experiment from HICs. This fact is exploited in the model of Sagert et al. (2009), where the bag constant has been chosen such that the strongly asymmetric matter in compact stars leads to almost pure quark stars with only a thin hadronic layer. These models are labeled as "STOSQxxxs," where "xxx" indicates the value of the bag constant $B^{1 / 4}$ in $\mathrm{MeV}$, and "s" stands for Sagert et al. The models of Nakazato, Sumiyoshi, and Yamada (2008) lie in a parameter range $\left(B^{1 / 4}=209 \mathrm{MeV}\right)$ where the critical density is much higher, such that the resulting NSs have only a small quark core. Strong interaction corrections are not used in this model. If a thermal pion gas is included in the hadronic phase, the transition to quark matter occurs at considerably higher densities (Nakazato, Sumiyoshi, and Yamada, 2008) due to the softening of the hadronic EoS by the pions. The latter models are labeled "STOSQxxxn" for the one with quarks and "STOSQxxxn $\pi$ " for the one with quarks and pions. Nakazato, Sumiyoshi, and Yamada (2013) calculated additional hybrid EoS tables for $B^{1 / 4}=162$ and $184 \mathrm{MeV}$. Because the corresponding maximum masses of $1.54 M_{\odot}$ and $1.36 M_{\odot}$ are well below the observed pulsar masses, in the following we consider only the table with $B^{1 / 4}=209 \mathrm{MeV}$ as a representative example of the hybrid EoSs of Nakazato, Sumiyoshi, and Yamada (2008, 2013). The thermodynamic properties are strongly influenced by the possible existence of quark matter at high densities and temperatures and, in particular, the phase transition can have an important effect on the dynamics of CCSNe (see Sec. VI.B.1).

\section{Compatibility with experimental and observational constraints}

In this section we compare the results of the general purpose EoSs with several constraints that have been introduced in Sec. IV. As can be seen from Table IV, most of the employed interactions give reasonable properties for compressibility, saturation density, and binding energy of symmetric matter, except that LS180 has a compressibility at the lower end of the allowed range and LS375 and TMA have values above the allowed range. However, some models give symmetry energies and slopes far off the best present constraints (see Fig. 13), i.e., the dependence of the EoS on $Y_{q}$ is probably not correctly described. DD2, SFHo, IUFSU, and FSUgold (2.1) are in the best agreement.

NS masses (see Figs. 14, 15, and Table III) probably represent the presently most reliable observational constraint on the compact star EoS. LS180 and the models based on FSUgold give too low masses and HS(IUFSU) is only marginally compatible. Note that the maximum mass depends only very little on the treatment of the inhomogeneous part of the EoS, such that all models with the same nuclear interaction give essentially the same maximum mass. This is not the case for the radii of intermediate-mass $\mathrm{NSs}$, which are more sensitive to the treatment of the crust. Here slight differences can be observed between HS(TM1), STOS, and FYSS, which are all based on the TM1 interaction; see Fig. 14 and Table III. Since the H\&W is not publicly available, in Fig. 14 the results of the model by Gulminelli and Raduta (2015) are plotted.
TABLE IV. Nuclear matter properties of the parametrizations for the nuclear interaction used in the general purpose EoS of Table III. Listed are the saturation density $n_{\text {sat }}$, binding energy $B_{\text {sat }}$, incompressibility $K$, skewness coefficient $Q=-K^{\prime}$, symmetry energy $J$, and symmetry energy slope coefficient $L$ at saturation density at zero temperature.

\begin{tabular}{lcccrrr}
\hline \hline $\begin{array}{l}\text { Nuclear } \\
\text { interaction }\end{array}$ & $\begin{array}{c}n_{\text {sat }} \\
\left(\mathrm{fm}^{-3}\right)\end{array}$ & $\begin{array}{c}B_{\text {sat }} \\
(\mathrm{MeV})\end{array}$ & $\begin{array}{c}K \\
(\mathrm{MeV})\end{array}$ & $\begin{array}{c}Q \\
(\mathrm{MeV})\end{array}$ & $\begin{array}{c}J \\
(\mathrm{MeV})\end{array}$ & $\begin{array}{c}L \\
(\mathrm{MeV})\end{array}$ \\
\hline SKa & 0.155 & 16.0 & 263 & -300 & 32.9 & 74.6 \\
LS180 & 0.155 & 16.0 & 180 & -451 & $28.6^{\mathrm{a}}$ & 73.8 \\
LS220 & 0.155 & 16.0 & 220 & -411 & $28.6^{\mathrm{a}}$ & 73.8 \\
LS375 & 0.155 & 16.0 & 375 & 176 & $28.6^{\mathrm{a}}$ & 73.8 \\
TM1 & 0.145 & 16.3 & 281 & -285 & 36.9 & 110.8 \\
TMA & 0.147 & 16.0 & 318 & -572 & 30.7 & 90.1 \\
NL3 & 0.148 & 16.2 & 272 & 203 & 37.3 & 118.2 \\
FSUgold & 0.148 & 16.3 & 230 & -524 & 32.6 & 60.5 \\
FSUgold2.1 & 0.148 & 16.3 & 230 & -524 & 32.6 & 60.5 \\
IUFSU & 0.155 & 16.4 & 231 & -290 & 31.3 & 47.2 \\
DD2 & 0.149 & 16.0 & 243 & 169 & 31.7 & 55.0 \\
SFHo & 0.158 & 16.2 & 245 & -468 & 31.6 & 47.1 \\
SFHx & 0.160 & 16.2 & 239 & -457 & 28.7 & 23.2 \\
\hline \hline
\end{tabular}

${ }^{a}$ The value for the symmetry energy $J$ is different from the value of 29.3 MeV in Lattimer and Swesty (1991). They computed $J$ as the energy difference between neutron and nuclear matter, whereas we are calculating $J$ as the second derivative with respect to $Y_{q}$ for symmetric matter at $n_{\text {sat }}$; see also Steiner, Hempel, and Fischer (2013).

This model is based on the same Skyrme interaction SkA. Note that the radius of a $1.4 M_{\odot}$ NS of Gulminelli and Raduta (2015) differs by about $1 \mathrm{~km}$ compared to the value of H\&W as given in Table III. In Sec. IV we outlined the difficulties in determining reliable NS radii and that presently no consensus on the allowed range of values can be obtained. Let us mention, however, that if small radii for $1.4 M_{\odot}$ NSs of the order 10-12 km were confirmed [as reported, e.g., by Özel et al. (2016)], then some of the hadronic models shown here

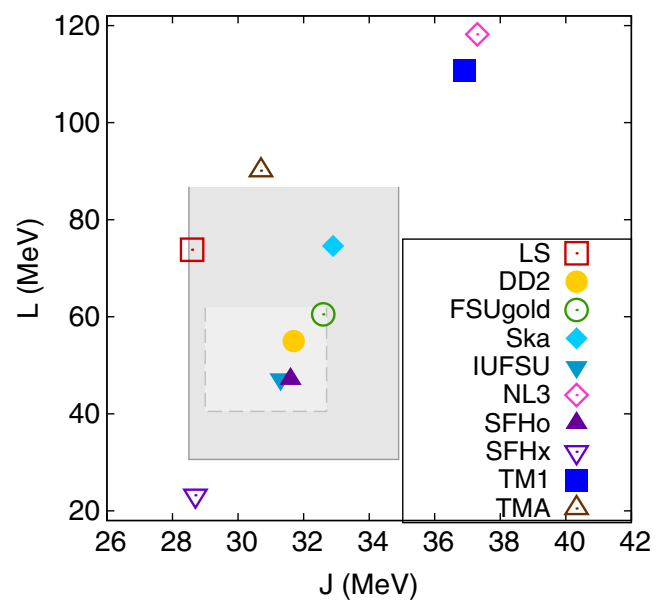

FIG. 13. The slope parameter of the symmetry energy $L$ vs the value of the symmetry energy $J$ at normal nuclear matter density. The light gray region is the constraint of Lattimer and Lim (2013), and the dark gray region is taken from Fig. 8. The different symbols show the values of the nucleon interactions of Table IV that are applied in the general purpose EoSs of Table III. FSU2.1 gives the same value as FSUgold. 


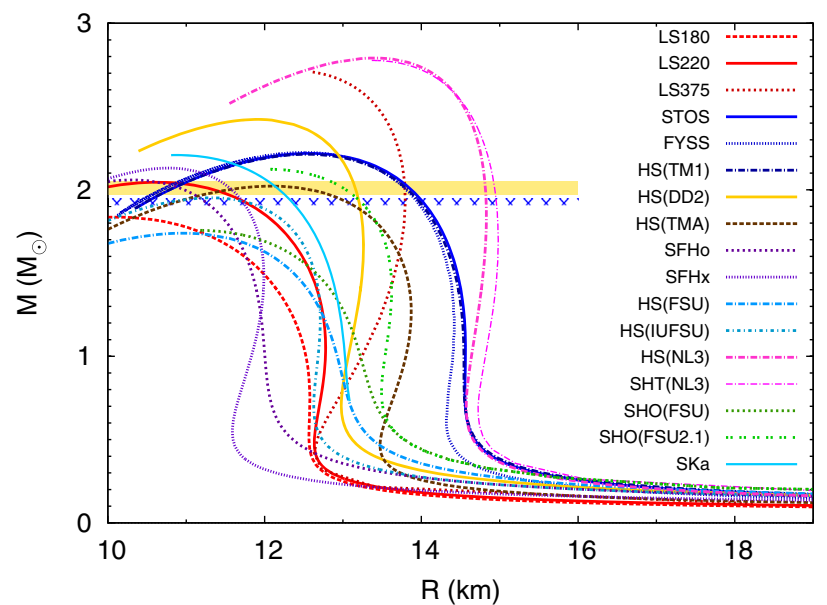

FIG. 14. Mass-radius relations of spherically symmetric NSs for the different EoSs that cover the full thermodynamic parameter range and include only nucleonic degrees of freedom; cf. Table III. The two horizontal bars indicate the two recent precise NS mass determinations, PSRJ1614-2230 (Demorest et al., 2010) (hatched blue) and PSR J0348 + 0432 (Antoniadis et al., 2013) (yellow). The curve labeled "SkA," although based on the same nuclear interaction, does not represent the $\mathrm{H} \& \mathrm{~W}$ EoS, but the model by Gulminelli and Raduta (2015).

could be excluded, in particular, those based on TM1, NL3, and LS375.

As can be seen from Fig. 15 and the data given in Table III, the NS maximum masses of most of the extended models with additional non-nucleonic degrees of freedom are not compatible with a $2 M_{\odot}$ star. $\mathrm{BHB} \Lambda \phi, \mathrm{STOS} \pi, \mathrm{STOSQ} 139 \mathrm{~s}$, and STOSQ145s are the only ones with NS maximum masses above $1.97 M_{\odot}$. As discussed, for hyperonic EoSs this is related to the hyperonic interactions used. Recent studies for cold NS EoSs overcome the maximum mass problem but these models for the interaction have not yet been applied to compute a complete EoS covering the whole range of temperature, hadronic charge fraction, and baryon density. Anyway, with increasing temperature the effect of the interactions

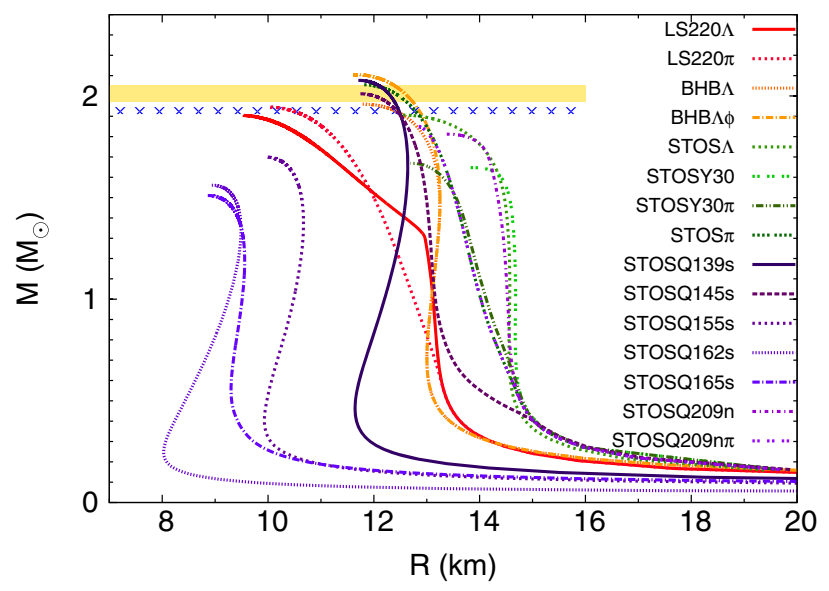

FIG. 15. Same as Fig. 14 for EoS models including additional degrees of freedom. The onset of additional degrees of freedom is visible as a change in the slope. becomes less important and most models agree qualitatively for the particle composition (cf. Fig. 12), whether or not compatible with a $2 M_{\odot}$ NS.

The situation becomes even more severe if additional constraints are included in the benchmarking. In the right panel of Fig. 16 the experimental flow constraint of Danielewicz, Lacey, and Lynch (2002) for the pressure as a function of baryon number density in symmetric nuclear matter is depicted. For neutron matter (left panel), the constraint from the $\chi \mathrm{EFT}$ calculation of Hebeler et al. (2013) is shown (cf. Sec. IV.B). These constraints are compared with the EoS of symmetric and neutron matter obtained at $T=0$ from the different models. It is obvious that none of the present models is perfectly compatible with the neutron matter results from Hebeler et al. (2013) below saturation density. However, the error band shown in the left panel of Fig. 16 is perhaps too small (see Fig. 6), rendering some of the models at least marginally compatible, such as DD2, SFHo, or FSUgold. The LS180 and LS220 models are in reasonable agreement with the constraint for $n_{B} \gtrsim 0.1 \mathrm{fm}^{-3}$ but give too low pressures at lower densities. The models SFHo and SFHx have been fitted to some NS radius determinations giving radii around $12 \mathrm{~km}$ for $1.4 M_{\odot}$ (Steiner, Lattimer, and Brown, 2010; Steiner, Hempel, and Fischer, 2013). In the extreme model SFHx, it was tried to make these as small as possible, within the employed class of RMF interactions. This is the reason why they have very low (SFHx) or moderate pressure (SFHo) for neutron matter around $n_{\text {sat }}$ and correspondingly low and moderate values of $L$. Similar observations as for neutron matter can be made from the comparison of the flow constraint with the EoS of symmetric nuclear matter: Many present models for the general purpose EoS seem to give a too large pressure at suprasaturation densities.

The neutron matter EoS is a crucial anchor point for the NS $\mathrm{EoS}$ and thus also of great significance for NS mergers. However, for CCSNe, matter is generally more symmetric and nuclear clusters are an important contribution to the subsaturation EoS. In Fig. 5 , several of the general purpose EoSs are included in a comparison with experimental data for cluster formation (see Sec. IV.A.4). The LS220 EoS shows a notable underproduction of $\alpha$ particles, and SHT(NL3) and $\mathrm{SHO}(\mathrm{FSU}$ 2.1) an overproduction at high temperatures. The other general purpose EoSs FYSS, SFHo, STOS, and HS(DD2), are more or less in reasonable agreement with the constraint. The current experimental data do not allow one to make further judgements about details of the medium modifications of nuclear clusters, e.g., to distinguish classical excluded-volume effects from quantum statistical Pauli blocking. For further discussion, see Hempel et al. (2015).

To conclude, there is not a single general purpose EoS that is compatible with all constraints, even though we considered only a few of them. However, from the purely nucleonic models SFHo, HS(DD2), and SHO(FSU2.1) are at least approximately consistent. From the EoS with additional degrees of freedom, only $\mathrm{BHB} \Lambda \phi$, which is also based on DD2, would be acceptable. Nevertheless these EoSs have further drawbacks and weaknesses: in the models based on HS [SFHo, HS(DD2), $\mathrm{BHB} \Lambda \phi]$ the treatment of light nuclei is not as advanced as in 

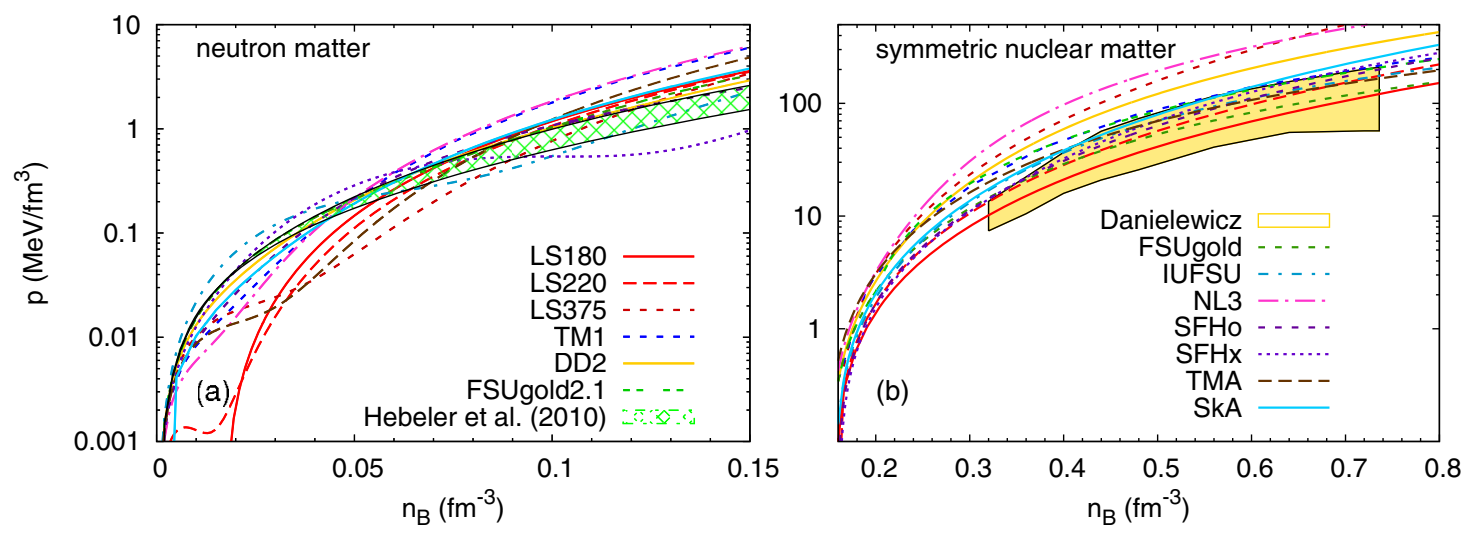

FIG. 16. Pressure as a function of baryon density within the EoS models listed in Table III for symmetric nuclear matter (right) and pure neutron matter (left). The results are compared with the theoretical calculation for neutron matter from Hebeler et al. (2013) and the experimental flow constraint from Danielewicz, Lacey, and Lynch (2002) for symmetric matter.

the quantum statistical model (see Sec. III.C.4) or generalized relativistic density functional (see Sec. III.C.5), and it does not include an explicit medium dependence of the nuclear binding energies of heavy nuclei, which one could extract from nucleons-in-cell-calculations (see Sec. III.C.6). Other EoSs (e.g., FYSS or SHO and SHT), which are more advanced in some of these aspects, do not fulfill constraints for the maximum mass or $L$. The SHO(FSU2.1) is compatible with the maximum mass constraint only because of an ad hoc modification of the pressure at high densities. Furthermore, of all possible light nuclei only the $\alpha$ particle is included in this model. Detailed nucleons-in-cell calculations are used for heavy nuclei, but only at intermediate to high densities, and light nuclei are not taken into account in this regime at all. The usage of different prescriptions in different regimes of $T$ and $n_{B}$ can also lead to problems in the thermodynamically consistent construction of transitions. The state of the art in modeling the general purpose EoS is thus not really satisfactory. There is still need for new general purpose EoSs that employ modern EDFs (or even beyond) with good nuclear matter properties, that tackle the problem of additional degrees of freedom at high densities and temperatures, and that give a detailed description of clustering at subsaturation densities.

\section{APPLICATIONS IN ASTROPHYSICS}

\section{A. Binaries and binary mergers}

Coalescing relativistic binary systems containing compact objects, either NSs or BHs, are interesting in the context of the EoS of dense and hot matter. They are likely to be important sources of detectable gravitational waves (GW) by advanced Laser Interferometer Gravitational-Wave Observatory (LIGO), Virgo, and Kamioka Gravitational Wave Detector (KAGRA), possibly before 2020. NS-NS and NS-BH mergers are believed to produce short gamma-ray bursts (sGRB). In addition, they may represent the major source for the main component of heavy $r$-process elements in the Universe; see, e.g., the recent reviews by Shibata and Taniguchi (2011), Faber and Rasio (2012), and Rosswog (2015) and references therein. All three, the GW signal, the sGRBs, and the $r$-process abundances, contain information on the EoS.
During the late inspiral phase of both NS-NS and NS-BH systems, NSs become tidally deformed to an extent that depends on the underlying EoS. Numerical models suggest that the GW frequency is very sensitive to the tidal deformation and thus to the underlying EoS (Shibata and Taniguchi, 2011; Faber and Rasio, 2012). However, the rate of NS-BH inspirals is uncertain (to date no such system has been observed), and the tidal effects from these systems are probably not visible for next-generation detectors since they occur at too high frequencies outside the range where the detectors are most sensitive (Pannarale et al., 2011). On the contrary, after the first detection of GW emission from a BH-BH merger (Abbott et al., 2016), there appears to be a good chance for binary NS mergers to be detected in the near future, and the tidal deformability has probably a strong enough impact on the GW signal (Read et al., 2013). Additional information on the EoS can be obtained from the postmerger phase, in cases where the EoS supports the formation of a hypermassive NS. The frequencies of NS normal modes after the merger are sensitive to the EoS and visible in the GW signal; see, e.g., Sekiguchi et al. (2011), Bauswein et al. (2012), and Takami, Rezzolla, and Baiotti (2014). Measurements of their frequencies could tightly constrain NS masses and radii since they are strongly correlated. Figure 17 illustrates the correlation between the dominant GW frequency in the postmerger phase, normalized to the total mass of the binary system, and the radius of a cold nonrotating NS with $M=1.6 M_{\odot}$. It could even be possible to give an estimate for the NS maximum mass (Bauswein, Baumgarte, and Janka, 2013; Bauswein, Stergioulas, and Janka, 2014; Bauswein and Stergioulas, 2015).

Fryer et al. (2015) and Lawrence et al. (2015) proposed another test of the EoS in binary NS mergers. Numerical models suggest that a sGRB is produced in such a merger only if a BH forms sufficiently fast. The fate of the core of the remnant, and thus the $\mathrm{BH}$ formation time, depends on the maximum mass supported, and thus on the EoS. Although other factors influence the maximum mass, for instance, the spin rate or the angular momentum distribution inside the core (Kastaun and Galeazzi, 2015), they suggested that it is possible to relate the existence of a sGRB to the maximum mass of a cold nonrotating NS. Assuming NS binary mergers to be the dominant source of a sGRB, a combined analysis of 


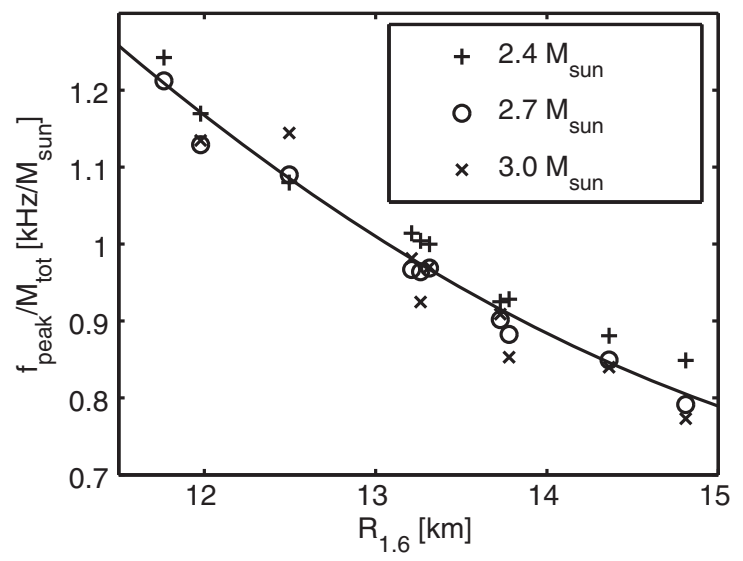

FIG. 17. Relation between the dominant GW frequency in the postmerger phase of a NS-NS binary merger event and the radius of a nonrotating NS with a gravitational mass of $M=1.6 M_{\odot}$. The frequency has been normalized with respect to the total mass of the system. Different symbols refer to different total masses, where equal-mass binaries are assumed. From Bauswein, Stergioulas, and Janka, 2016 with kind permission of The European Physical Journal (EPJ).

the observed burst rate and the total merger rate with GW detectors would then allow for constraining the EoS. A different model for a sGRB invokes a supramassive magnetar as the central engine instead of a $\mathrm{BH}$. Fan, Wu, and Wei (2013) extracted the NS maximum mass within this scenario using GRB afterglow observations, where the abrupt decline of the x-ray plateau is interpreted as the collapse to a $\mathrm{BH}$.

Compact binary mergers eject initially extremely neutronrich matter, and have therefore already very early been identified as possible sources of $r$-process elements in the Universe (Lattimer and Schramm, 1974). Recent calculations show that the conditions are favorable for producing a robust $r$-process abundance pattern of heavy nuclei that is close to the solar (Rosswog, 2015). The $r$-process production rates, the final abundances, and the amount of ejected material depend on the chemical composition and the thermodynamic conditions in the ejecta, and thus on the EoS; see, e.g., Bauswein, Goriely, and Janka (2013), Wanajo et al. (2014), and Sekiguchi et al. (2015). The radioactive decay of the freshly produced $r$-process elements should produce an electromagnetic transient, called a kilonova or macronova. Recently, a first candidate event has been reported, associated with the sGRB 130603B (Tanvir et al., 2013). Macronova signatures have also been found for sGRB 060614 (Yang et al., 2015) and sGRB 050709 (Jin et al., 2016). Assuming an almost equal-mass NS-NS merger as a source of sGRB 130603B, Hotokezaka, Kyutoku et al. (2013) showed that an EoS giving $R_{1.35} \lesssim 13.5 \mathrm{~km}$ is preferred in order to match the inferred relatively large values of ejecta masses and velocities. This result, however, depends strongly on the initial mass ratio and the type of merger. Although expected to occur much less frequently, a compact binary merger, NS-NS or NS-BH, with an initial mass ratio substantially different from unity naturally produces high ejecta masses and velocities (Oechslin, Janka, and Marek, 2007). Kawaguchi et al. (2016) analyzed sGRB $130603 \mathrm{~B}$ within this scenario and found that a NS radius above $11 \mathrm{~km}$ is favored. It might be possible to obtain reliable information about the underlying EoS from the final nucleosynthesis outcome with more observations and more detailed simulations.

\section{B. Core-collapse supernovæ}

\section{Dynamics}

The dynamics of CCSNe results from a complex interplay between hydrodynamics, neutrino transport, weak interactions, and the EoS. The general expectation, called Mazurek's law, is that due to the strong feedback, a small modification of one of the ingredients does not qualitatively change the dynamics (Lattimer and Prakash, 2000). However, the quantitative differences induced by different EoS can be large enough to govern the presence or absence of an explosion (Janka, 2012a; Suwa et al., 2013).

Since in the early phase electron pressure dominates and later on the collapse proceeds homologously, the dynamics of the infall epoch has only a mild direct dependence on the baryonic part of the EoS. It is sensitive to the electron fraction $Y_{e}$ and the entropy. Changes of $Y_{e}$ result from electron captures (EC) on nuclei and free protons and therefore depend on the composition, in particular, the abundances and the mass and charge of the appearing nuclei. The mass of the inner core at bounce $M_{i c}$ is roughly proportional to $\left\langle Y_{L_{e}}^{2}\right\rangle$, the mean fraction of trapped leptons squared (Lattimer, Burrows, and Yahil, 1985), which is fixed and given by $Y_{e}$ at the moment where neutrino trapping sets in.

Many studies, including those employing a statistical model for the EoS, use the single nucleus approximation within the simplified EC rates from Bruenn (1985) in order to determine the evolution of $Y_{e}$. In this simple model, the reaction $Q$ value, determining the phase space available for the capture reaction, is approximated by the difference in proton and neutron chemical potentials,

$$
\hat{\mu} \equiv-\mu_{Q}=\mu_{n}-\mu_{p},
$$

which strongly depends on the EoS. Roughly, the larger $\mu_{Q}$ the larger the electron-capture rate. This quantity is illustrated for different EoSs in Fig. 18. An entropy per baryon of $s=1$

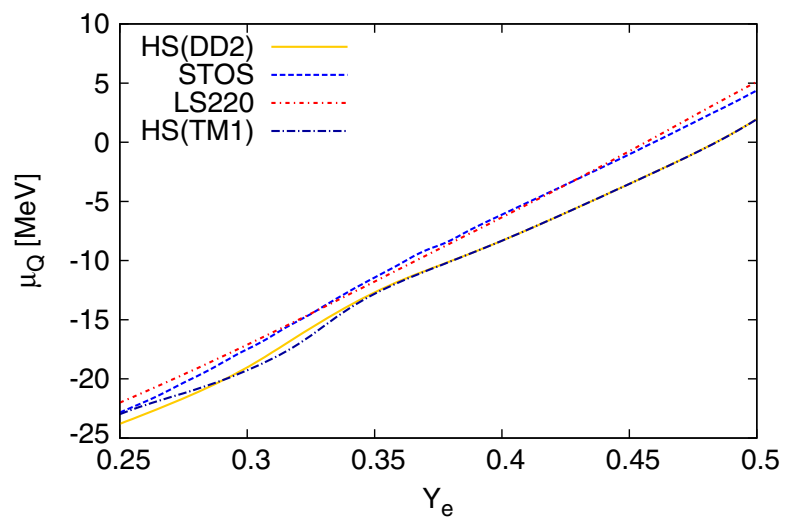

FIG. 18. A comparison of the values of charge chemical potential $\mu_{Q}$ for different EoSs and typical thermodynamic conditions in the infall epoch. The proton fraction has been varied at constant entropy per baryon $s=1$ and constant baryon number density $n_{B}=10^{-3} \mathrm{fm}^{-3}$. 
has been chosen, corresponding to a typical value before shock formation and a baryon number density of $n_{B}=10^{-3} \mathrm{fm}^{-3}$. It is evident that not the saturation properties of cold matter within the EoS are relevant to determine $\mu_{Q}$, but the treatment of inhomogeneous matter, i.e., nuclei. For instance, the difference between the model HS(TM1) and STOS, using the same interaction and having the same saturation properties, is much larger than between HS(DD2) and HS(TM1) which have very different nuclear properties but share the same treatment of nuclei. This was already pointed out by Lattimer and Prakash (2000), where it was shown within a simple analytical model that $\mu_{Q}$ was much more sensitive to surface energies than to the bulk symmetry energy.

The EC rates also depend on entropy. Already at the stage of the progenitor small differences in entropy arise between the EoS employed for core collapse and the progenitor model, in general based on a nuclear reaction network. In addition, in the STOS EoS, the entropy contribution of the thermal motion of heavy nuclei is missing (see Sec. V.D.1.c), reducing the entropy and thus underestimating the deleptonization.

Another effect is that within the description of Bruenn (1985), the EC rate strongly decreases with the neutron number $N$. Since in general the distribution of nuclear abundances is large in statistical models, the average heavy nucleus can be very different from the single heavy nucleus in the SNA (see Sec. III.C.2 and Fig. 10). In HS(TM1), for instance, $N$ is in general smaller than in STOS, where the average mass of heavy nuclei is overestimated (related to the Thomas-Fermi approximation and the used value for the gradient energy coefficient, see Sec. V.D.1.c), leading to a higher EC rate in the domain where EC on nuclei is dominant (Hempel et al., 2012).

A remark of caution is in order here. It was clearly demonstrated that it is not sufficient to use one average heavy nucleus in order to determine the global EC rate on nuclei, because of shell effects on individual rates which are not correctly reproduced within this approximation; see, e.g., Langanke et al. (2003) and Juodagalvis et al. (2010). Better calculations for individual rates, attenuating, in particular, the strong suppression of EC on neutron-rich nuclei present in the (Bruenn, 1985) prescription, change the infall evolution and the bounce properties too. The individual EC rates can change by an order of magnitude from one nucleus to another which has an important effect on the final $Y_{L_{e}}$; see, e.g., Hix et al. (2003), Furusawa, Nagakura et al. (2013), Raduta, Gulminelli, and Oertel (2016), and Sullivan et al. (2016). The impact on the dynamics of a full CCSN simulation is still to be seen.

Concerning the direct impact of the EoS and the nuclear composition, Hempel et al. (2012) performed a detailed analysis of the different stages during the infall epoch comparing the LS180, STOS, and HS(TM1) EoSs confirming within a simulation some of the previously discussed effects. At the time of bounce small differences can be observed between the different EoSs. An important effect is that a low central $Y_{e}$ is correlated with a low core mass; see, e.g., Sumiyoshi et al. (2005), Janka (2012a), Hempel et al. (2012), Steiner, Hempel, and Fischer (2013), and Suwa et al. (2013). A smaller core mass at bounce leads to a weaker shock forming closer to the center. Naively, this would lead to a situation less favorable for an explosion. In addition, more mass overlays the core in this case rendering an explosion still more difficult. However, different effects compete. For instance, a stronger deleptonization leads to a higher neutrino luminosity which in turn heats the shock more strongly. Therefore no clear statement is possible and most studies in spherical symmetry conclude on a minor effect of the EoS on the overall dynamics (Sumiyoshi et al., 2005; Hempel et al., 2012; Janka, 2012a; Steiner, Hempel, and Fischer, 2013; Suwa et al., 2013; Fischer, Hempel et al., 2014; Togashi et al., 2014). In particular, it is difficult to relate single nuclear matter parameters of the EoS to CCSN dynamics, unless one compares EoSs that show very pronounced differences. The fact that the existing general purpose EoSs often differ in several properties, also because of correlations among different parameters (see Sec. IV), makes systematic investigations difficult. In addition, as illustrated, the treatment of inhomogeneous matter, properties of nuclei, and thermal effects are found to be equally important as the very neutron-rich and dense part of the EoS (Fischer, Hempel et al., 2014). This might be different for $\mathrm{BH}$ formation, where the PNS maximum mass is decisive (cf. Sec. VI.B.3).

A more compact and more rapidly contracting PNS resulting from a "softer" $\operatorname{EoS}^{6}$ generally seems to be favorable for explosions in multidimensional simulations. In particular, neutrinos are emitted with higher fluxes and higher energies (Marek, Janka, and Müller, 2009). This not only enhances neutrino cooling in $2 \mathrm{D}$, but favors the formation of more violent hydrodynamical instabilities and stronger convection (Janka, 2012a; Suwa et al., 2013). This is illustrated in Fig. 19, where the evolution of the PNS radius (upper panel) and the shock radius (lower panel) is shown for three different EoSs within a 2D simulation by the Garching group. It is evident that in this case the EoS decides upon the explosion.

There might be an imprint of the EoS on the neutrino and $\mathrm{GW}$ signal. For instance, the faster deleptonization during the infall epoch leads to an enhancement in the neutrino luminosity at early times. The higher neutrino fluxes and higher energies of the emitted neutrinos from a more compact PNS should lead to differences in the neutrino spectra too. From a galactic supernova, these differences should indeed be observable with present detectors; see Sumiyoshi et al. (2005), Marek, Janka, and Müller (2009), and Suwa et al. (2013). A more compact PNS leads to higher frequencies and larger amplitudes for the emitted GW too (Marek, Janka, and Müller, 2009; Scheidegger et al., 2010). However, the EoS is not the only varying parameter, for example, the unknown progenitor structure or the treatment of neutrino transport can induce modifications in the evolution of the CCSN, such that it seems very difficult to unambiguously identify one particular effect.

Recent 1D studies of CCSNe with non-nucleonic degrees of freedom focus on $\mathrm{BH}$ formation (see Sec. VI.B.3), where

\footnotetext{
${ }^{6}$ Please note that the terms "soft" and "stiff" for the EoS are not necessarily related to the incompressibility of cold symmetric nuclear matter or any other nuclear matter parameter due to thermal effects. It means here simply that the overall PNS is more compressible for a soft EoS.
} 

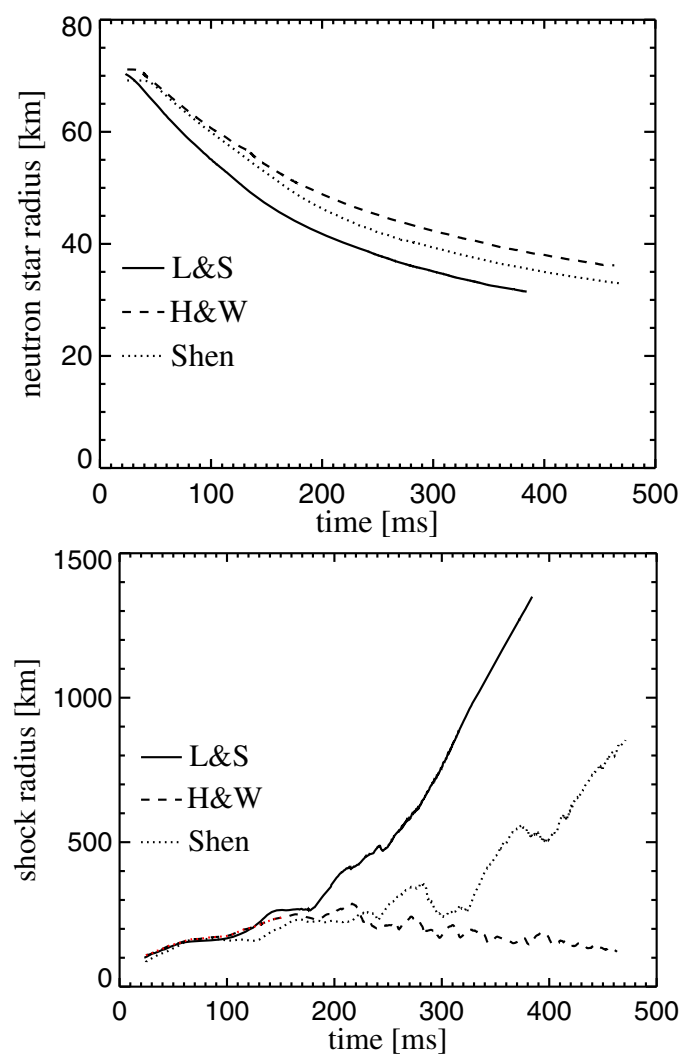

FIG. 19. The top panel shows the evolution of the PNS's radius for a two-dimensional CCSN simulation employing the LS180, the H\&W, and the STOS EoSs. The bottom panel shows the evolution of the shock radius. From Janka, 2012 b.

sufficiently high temperatures and densities are reached so that these degrees of freedom are expected to have a notable effect on the dynamics. An exceptional case for regular supernova explosions, i.e., without BH formation, could be the onset of non-nucleonic degrees of freedom via a strong first-order phase transition occurring close to saturation density. As pointed out by Gentile et al. (1993) and confirmed by Sagert et al. (2009) with detailed Boltzmann neutrino transport, in spherically symmetric simulations a second shock can be formed as a direct consequence of a phase transition to quark matter. This second shock was found to be strong enough to unbind the outer layers once it merges with the standing accretion shock (Sagert et al., 2009). In this way, a CCSN explosion is triggered due to the phase transition to quark matter. Furthermore, the passage of the second shock leads to a second neutrino burst that is dominated by electron antineutrinos, measurable with present-day detectors (Dasgupta et al., 2010).

However, the NS maximum masses of the EoSs applied by Sagert et al. (2009) (STOSQ162s, STOSQ165s) are well below $2 M_{\odot}$, and thus ruled out by NS observations. In the subsequent works exploring this scenario [see, e.g., Sagert et al. (2010), Fischer et al. (2011, 2012), Nakazato, Sumiyoshi, and Yamada (2013), and Fischer, Hempel et al. (2014)], explosions could not be obtained if the maximum mass of the employed EoS is sufficiently high. It is clear that the required stiffening in the quark phase to reach $2 M_{\odot}$ typically does not allow for a strong first-order phase transition that seems to be necessary in this scenario to trigger explosions. This can be related to the so-called masquerade problem (Alford et al., 2005), known for the mass-radius relation of NSs where quark matter could behave very similar to hadronic matter. On the other hand, it has not been shown yet that a SN explosion induced by a phase transition is ruled out by the latest pulsar mass measurements.

Overall, there are still many uncertainties and open questions about the CCSN explosion mechanism, such as the dependency on the progenitor, effects of magnetic fields and rotation, numerical convergence, the strength and scale of intrinsic multidimensional hydrodynamic effects (turbulence, convection, SASI, etc.), or an accurate treatment of neutrino interactions and their transport (Mezzacappa, 2005; Kotake, Sato, and Takahashi, 2006; Janka et al., 2007; Ott, 2009; Janka, 2012a; Burrows, 2013). The EoS is one of them. Its role is not yet fully understood, partly due to the high complexity of the system and the interplay of all the aforementioned aspects.

\section{PNSs, neutrino-driven winds, and nucleosynthesis}

A neutrino-driven wind (NDW) is the emission of a lowdensity, high entropy baryonic gas from the surface of a newly born PNS in a CCSN. It is driven by energy deposition of neutrinos emitted from deeper layers and sets in after the launch of the SN explosion. It remains active in the first seconds up to minutes. The NDW is of great importance for the nucleosynthesis of heavy elements, as it has been considered as one of the most promising sites for the $r$ process; see, e.g., the review by Arcones and Thielemann (2013). However, previous sophisticated long-term simulations of CCSNe (Fischer et al., 2010; Hüdepohl et al., 2010) have shown that the matter emitted in the NDW is generally proton rich, allowing only for the so-called $\nu p$ process (Fröhlich et al., 2006; Fröhlich, Martínez-Pinedo et al., 2006; Arcones, Janka, and Scheck, 2007; Roberts, Woosley, and Hoffman, 2010; Arcones and Thielemann, 2013), which is not able to produce the most heavy nuclei.

Martínez et al. (2012), Roberts, Reddy, and Shen (2012), and Roberts (2012) realized that these long-term simulations of the PNS deleptonization phase and the NDW neglected the effect of nuclear interactions in the charged-current (CC) interaction rates of neutrinos with unbound nucleons. As outlined in Sec. III.B.2, the nucleon single-particle energies within mean-field models can be written as the sum of a kinetic part which has the form of a free gas, depending on an effective mass, and an interaction potential $V_{i}$,

$$
E_{i}=E^{\mathrm{kin}}\left(m_{i}^{*}\right)+V_{i}
$$

The difference of neutron and proton energies, which determines the energy available for (anti)neutrinos from $\mathrm{CC}$ reactions, depends on $\Delta V=V_{p}-V_{n}$. In asymmetric matter within the hot PNS, $\Delta V$ can be as large as $50 \mathrm{MeV}$. It does not affect neutral current reactions, unless $\Delta V$ carries an additional energy dependence and one has an inelastic reaction.

The simulations of Martínez-Pinedo et al. (2012), Roberts, Reddy, and Shen (2012), and Roberts (2012) found that including $V_{i}$ correctly modifies the evolution of neutrino spectra and the deleptonization of the PNS. $\Delta V$ induces an 
increase of the antineutrino energies and a decrease of the neutrino energies. This leads to slightly neutron-rich conditions in the NDW. Because $\Delta V$ is related to the potential part of the symmetry energy (Hempel et al., 2015), (anti)neutrino spectra and the conditions in the NDW are sensitive to the isospin dependence of the EoS.

Although the correct treatment of mean-field effects in the $\mathrm{CC}$ reactions favors less proton-rich conditions in the NDW than previously determined, the values of $0.46<Y_{e}<0.5$ obtained in the simulations ${ }^{7}$ of Roberts, Reddy, and Shen (2012) and Martínez-Pinedo, Fischer, and Hüther (2014) with different EoSs are still not low enough for a robust $r$-process nucleosynthesis. Similar conclusions have been obtained within the QCD phase transition scenario (see Sec. VI.B.1), where again a slightly proton-rich NDW is produced, leading to a weak $r$ process (Nishimura et al., 2012). However, there are still many open questions, e.g., the employed approximations in the neutrino transport and reaction rates, a possible progenitor dependence, or the role of light nuclei in the envelope of PNSs.

For instance, Arcones et al. (2008) found that the envelope composition is dominated by nucleons, deuterons, tritons, and $\alpha$ particles, in agreement with other works; see, e.g., Sumiyoshi and Röpke (2008), Hempel et al. (2012), and Fischer, Hempel et al. (2014). Arcones et al. (2008) showed that light nuclei other than the $\alpha$ particle (mainly deuterons and tritons) lead to a small reduction of the average energy of the emitted electron neutrinos.

The long-term deleptonization and cooling of the PNS (Prakash et al., 1997; Pons, Steiner et al., 2001) contains further interesting aspects related to the EoS. Within the delayed BH formation scenario, the loss of thermal energy and, in particular, the deleptonization destabilizes the PNS (cf. Sec. VI.B.3). For an exploding CCSN, convection in the subsequently contracting and cooling PNS is very sensitive to the EoS, as in the early postbounce phase. Since convection depends strongly on the variation of pressure with lepton fraction $Y_{L_{e}}$ at constant $n_{B}$, the EoS dependence is mainly characterized by the symmetry energy (Roberts et al., 2012). They showed that the symmetry energy leaves an imprint in the neutrino count rates of present-day neutrino detectors for a galactic CCSN.

In the simulation of Suwa (2014) the long-term evolution of the PNS was followed in a self-consistent manner starting from its birth in the CCSN up to $\sim 70 \mathrm{~s}$. It is the first time that such a simulation entered the regime of conditions where the formation of the crust is expected.

\section{Black hole formation}

In a stellar core-collapse event a NS is formed if the exploding star successfully unbinds the ejected material after bounce. In a so-called failed CCSN, the outcome may equally be a stellar mass $\mathrm{BH}$ if the expanding shock is not able to break through the infalling material and accretion pushes the

\footnotetext{
${ }^{7}$ Note that the $Y_{e}$ values are different compared with the values reported by Roberts, Reddy, and Shen (2012), due to a previous computational error which was later corrected (Roberts, presentation at the MICRA workshop in Trento, 2013).
}

PNS over its mass limit on the time scale of seconds. Alternatively, there can be a delayed $\mathrm{BH}$ formation process, where either the cooling PNS becomes unstable or the fall back of ejecta causes the collapse to a $\mathrm{BH}$ in the minutes following the bounce. Numerical studies of $\mathrm{BH}$ formation in core collapse have a long history; see, e.g., O'Connor and Ott (2011) and references therein.

All scenarios have in common that the formation of an apparent horizon is accompanied by a significant drop in neutrino luminosity since most of the neutrino emitting material is swallowed up by the $\mathrm{BH}$. The GW signal could be interesting too in this context, being sensitive to oscillations in the hot PNS (Cerdá-Durán et al., 2013) and thus to the EoS. Pons, Steiner et al. (2001) and Nakazato et al. (2010) demonstrated that for a galactic event the time between bounce and $\mathrm{BH}$ formation $t_{\mathrm{BH}}$ is possibly observable from the neutrino signal in the Super-Kamiokande detector. However, the evolution of the core collapse and $t_{\mathrm{BH}}$ crucially depends also on the progenitor structure. O'Connor and Ott (2013) showed that it might be possible to get information about the compactness of the core of the progenitor star from the neutrino spectra and luminosities, which would allow one to disentangle the effects of the progenitor and of the EoS to some extent. In addition, rotation can strongly change not only the time until $\mathrm{BH}$ formation, but also the neutrino signal itself (Sekiguchi and Shibata, 2011). In particular, neutrino emission continues on a reduced level well after $\mathrm{BH}$ formation from the newly formed accretion disk, rendering the interpretation of the neutrino signal less obvious.

The EoS, as well, strongly influences the time until $\mathrm{BH}$ formation, since it determines the maximum mass supported by the hot PNS. There are two different physical mechanisms leading to the final gravitational instability: either the collapse is accretion induced or due to deleptonization and/or cooling.

In the latter case, it is not necessarily the reduced thermal pressure which destabilizes the cooling PNS, but deleptonization. In the hot PNS, due to the presence of trapped neutrinos, matter is very lepton rich and $Y_{e}$ can be as high as 0.4 (Prakash et al., 1997; Pons et al., 1999). This leads to a suppression of additional degrees of freedom containing strangeness (Prakash, Cooke, and Lattimer, 1995), such as hyperons, a kaon condensed phase, and/or a delayed phase transition to quark matter (see also Sec. V.D). Consequently, metastable PNSs could exist, whose maximum mass is above that of cold, $\beta$-equilibrated NS (Prakash et al., 1997). During the deleptonization of the hot PNS, the fraction of hyperons or quarks increases, eventually inducing a loss of stability and a collapse to a BH; see, e.g., Keil and Janka (1995), Baumgarte et al. (1996), and Pons, Steiner et al. (2001).

For an accretion-induced collapse in a failed CCSN, $t_{\mathrm{BH}}$ is too short for considerable deleptonization. Here the PNS cannot support the additionally accreted mass. The sensitivity of $t_{\mathrm{BH}}$ to the EoS has been demonstrated in many studies; see, e.g., Sumiyoshi, Yamada, and Suzuki (2007), Fischer et al. (2009), O'Connor and Ott (2011), and Suwa et al. (2013). However, there is no straightforward relation between any property of a given EoS, for instance, the maximum mass of a cold $\beta$-equilibrated NS, and the PNS mass at the onset of $\mathrm{BH}$ collapse. The reason is that $\mathrm{BH}$ formation is a dynamical process, and the temperature, density, and $Y_{e}$ distribution in 
the hot PNS depends on many factors and is, in particular, very different from that of a cold and $\beta$-equilibrated NS. Hempel et al. (2012) and Steiner, Hempel, and Fischer (2013) proposed an interesting ansatz: employing an extensive set of nuclear EoSs in simulations with a $40 M_{\odot}$ solar metallicity (Woosley and Weaver, 1995) progenitor, it was shown that for the given setup, $t_{\mathrm{BH}}$ can be correlated with the maximum mass of a $\beta$-equilibrated isentropic PNS at $s=4$. However, the evolution of a CCSN does not depend only on the EoS, but on many other factors (as discussed previously) which makes it difficult to unambiguously relate $t_{\mathrm{BH}}$ to the EoS.

Failed $\mathrm{CCSNe}$ have larger accretion rates than their exploding counterparts, such that higher temperatures and densities are reached within the PNS. As discussed in Sec. V.D, this could lead to a sustained production of additional degrees of freedom such as quarks or hyperons. Subsequently, the EoS is softened, supporting less mass and reducing $t_{\mathrm{BH}}$ compared with a purely nuclear EoS; see, e.g., Ishizuka et al. (2008), Sumiyoshi et al. (2009), Nakazato, Sumiyoshi, and Yamada (2010), Nakazato et al. (2012), Peres, Oertel, and Novak (2013), and Char, Banik, and Bandyopadhyay (2015). Since these non-nucleonic degrees of freedom appear only deep inside the PNS, apart from $t_{\mathrm{BH}}$ no considerable difference in the neutrino signal is to be expected with respect to a purely nuclear EoS, except if the appearance of additional particles is accompanied by a phase transition (Nakazato, Sumiyoshi, and Yamada, 2010; Peres, Oertel, and Novak, 2013).

Thus, although it is a promising field, there is still work needed before we can conclude from the neutrino signal on the EoS. We emphasize again that such difficulties are rather typical for observables of CCSNe (cf. Sec. VI.B.1).

\section{SUMMARY AND CONCLUSIONS}

Describing properties of matter in compact stars, their formation and merger processes is a very challenging task. The wide range of densities, temperatures, and charge fractions to be covered includes extreme values out of reach in terrestrial experiments. Therefore, one has to rely on theoretical modeling. However, dense hadronic and quark matter is difficult to describe since the many-body problem with strongly interacting particles has to be solved. In this review, we discussed theoretical and phenomenological approaches to address these difficulties.

In addition, we reviewed constraints on the EoS that have been obtained from experiments, astrophysical observations, and $a b$ initio calculations. Let us mention here some particularly important constraints. First, the recent observation of two NSs with precisely and reliably determined masses of about $2 M_{\odot}$ has triggered intensive discussions on the composition of matter in the central part of NSs. These results put strong constraints on the high-density, low-temperature part of the EoS. Second, considerable progress has been made in recent years concerning theoretical $a b$ initio calculations of pure neutron matter up to roughly saturation density, thus constraining the neutron-rich part of the EoS in this density regime. Third, laboratory experiments are beginning to converge to a common prediction for the symmetry energy and its slope around the saturation density.
There exist plenty of EoSs for cold NSs. To a lesser extent this still holds for EoSs for homogeneous hot matter in PNSs. In this review, the emphasis has been put on EoSs that cover the entire range of thermodynamic variables, which is relevant for simulations of CCSNe and compact binary mergers. They are much more rare, although in recent years much effort has been devoted to the development of new models, focusing on two aspects. First, the treatment of cluster formation and inhomogeneous matter at low densities and temperatures has been considerably improved. It was realized that light nuclei, which were previously ignored, can be important. Subsequent CCSN simulations have shown that differences in the cluster description induce differences in the dynamical evolution which are as important as those arising from different nuclear interaction models. Second, improved interactions and additional particles have been considered for the high density and temperature part, such as hyperons, mesons, and quarks. These non-nucleonic degrees of freedom influence, in particular, black hole formation, and NS-NS and NS-BH mergers.

Despite all efforts, there is much room for improvement. The cluster treatment is often based on a purely phenomenological description with several approximations and simplifications (see Sec. III.C). The interaction models employed cannot be considered definite. For instance, no presently existing model is consistent with all available constraints. However, it is clear that some of the constraints have to be regarded with care. Not all of them have the same reliability as the $2 M_{\odot}$ NS mass measurements; see the discussion in Sec. IV.

The quality of constraints is expected to improve in the future. For example, the current efforts to determine NS radii with an unprecedented $5 \%$ precision by projects such as ATHENA+, NICER, LOFT, and others promise rich information regarding the inner NS structure. GW astronomy has the potential to give new and completely independent insight into compact stars and their underlying EoS. New laboratory experiments and experimental facilities such as RIKEN, FRIB, FAIR, or NICA will provide new constraints for high density matter. We emphasize that all available general purpose EoSs are based on phenomenological approaches due to the computational and conceptual complexity of more microscopic methods. In the future, the increase in computational power is likely to allow the latter to provide EoSs suitable for astrophysical simulations too.

To conclude this review, let us mention, without claiming to be exhaustive, some important questions, which have to be addressed to develop a more realistic EoS. Because of the large range of variables covered in simulations, very different domains are encountered.

- Can we obtain a reliable description of all basic baryonic few-body interactions?

- How and under which conditions do non-nucleonic degrees of freedom appear?

- When does nuclear matter deconfine?

- Can we develop a QCD-based framework that covers the relevant range of variables?

- How do we better treat spatially inhomogeneous matter and cluster formation?

- How do we describe phase transitions consistently? 
Answers to any of these problems will result in better models for the astrophysical EoS and will help to understand various fundamental phenomena such as the composition and dynamics of NSs, the explosion mechanism of CCSNe, the threshold to $\mathrm{BH}$ formation, the nature of gamma-ray bursts, or the origin of heavy elements and the related galactical chemical evolution. In turn, these astrophysical insights are potentially relevant for the analysis of relativistic HICs and possibly for the search of QCD phase transitions.

\section{ACKNOWLEDGMENTS}

We thank S. Furusawa, C. Ishizuka, K. Nakazato, and K. Sumiyoshi for providing the data of their EoS, T. Fischer for providing Fig. 1, M. Fortin for providing Fig. 7, and A. Perego for providing Fig. 2. We are grateful to A. Botvina, N. Buyukcizmeci, I. Mishustin, and A. Raduta for providing us with the data for Fig. 10. We appreciate the thoughtful and encouraging comments of T. Fischer, T. Gaitanos, C. Miller, G. Röpke, J. Schaffner-Bielich, and H. Wolter to early versions of this review. This work was partially funded by NewCompStar, COST Action MP1304. M. O. received financial support from the Agence Nationale de la Recherche Project SN2NS No. ANR-10-BLAN-0503. M. H. is supported by the Swiss National Science Foundation. T. K. is grateful for support from the Polish National Science Centre (NCN) under Grant No. UMO-2013/09/B/ST2/01560. S. T. is supported by the Helmholtz Association (HGF) through the Nuclear Astrophysics Virtual Institute (No. VH-VI-417) and by the DFG through Grant No. SFB1245.

\section{APPENDIX: RESOURCES}

\section{EoS databases}

Here we present a list of publicly available EoS databases. Many authors provide their EoSs online, among them many of those presented in Sec. V.D.

- Lattimer and Swesty (1991) http://www.astro.sunysb.edu/dswesty/lseos.html

The original LS EoSs are available in the form of a computer program. Several authors have generated tabulated versions from it that can be found in the other databases. The various tables can differ in the range of thermodynamic variables covered and the details of the underlying calculation.

http://www.astro.sunysb.edu/lattimer/EOS/main.html

Four unpublished general purpose EoSs are tabulated at this web site, where, according to their names, three of them are based on the Skyrme interactions SKI', SKa, and $\mathrm{SKM}^{*}$, and the fourth has an incompressibility of $370 \mathrm{MeV}$.

- Sumiyoshi (1998)

http://user.numazu-ct.ac.jp/ sumi/eos/index.html

[Hosts data of Ishizuka et al. (2008) as well].

- Shen and Horowitz (2010)

http://cecelia.physics.indiana.edu/gang_shen_eos/

- Hempel (2011)

http://phys-merger.physik.unibas.ch/ hempel/ eos.html
The resources listed provide data in the full space of temperature, density, and asymmetry. A Web site that offers EoS tables for NS matter is as follows:

- Potekhin and Haensel (2013) http://www.ioffe.ru/astro/NSG/nseoslist.html

Different groups maintain online EoS collections with additional features. Here we provide the links with a small synopsis as found at the corresponding Web sites:

- STELLARCOLLAPSE.ORG

O'Connor and Ott (2008)

http://www.stellarcollapse.org

“..., a website aimed at providing resources supporting research in stellar collapse, core-collapse supernovae, neutron stars, and gamma-ray bursts."

STELLARCOLLAPSE.ORG provides not only tabulated EoS data but hosts valuable resources, information, and freely available open source code for stellar collapse and related phenomena. The available open source codes are listed in Sec. 2 of the Appendix.

- COMPOSE

Klähn, Oertel, and Typel (2013) and Typel, Oertel, and Klähn (2015)

http://compose.obspm.fr

"The online service COMPOSE provides data tables for different state-of-the-art equations of state (EoSs) ready for further usage in astrophysical applications, nuclear physics and beyond."

COMPOSE has been developed by us with support of the ESF-funded network CompStar, and the successive COST Action MP1304, NewCompStar. The community behind these research networks consists of EoS developers and users. A driving idea behind COMPOSE is not only to host a wide range of EoS data but to provide it in a flexible, multiple purpose, and reusable data format applicable for NS and CCSN EoSs. The LORENE library (Gourgoulhon et al., 2016) is COMPOSE compatible.

- EOSDB

Ishizuka et al. (2015)

http://aspht1.ph.noda.tus.ac.jp/eos/index.html

"Our aim is to summarize and share the current information on nuclear EoS which is available today from theoretical/experimental/observational studies of nuclei and dense matter."

EOSDB offers the possibility to search, compare, and graphically represent nuclear matter EoSs and related quantities online.

\section{Open source simulation software}

EoSs are a crucial input to many astrophysical simulations. We present a list of publicly available codes treating problems related to this review.

- LORENE

Gourgoulhon et al. (2016)

http://www.lorene.obspm.fr

“... a set of $\mathrm{C}++$ classes to solve various problems arising in numerical relativity, and more generally in computational astrophysics." 
- RNS

Stergioulas (1996)

http://www.gravity.phys.uwm.edu/rns/

“... constructs models of rapidly rotating, relativistic, compact stars using tabulated equations of state which are supplied by the user."

- STELLARCOLLAPSE.ORG

O'Connor and Ott (2008)

http://www.stellarcollapse.org

offers several codes:

(1) GR1D:

spherically symmetric code for stellar collapse to neutron stars and black holes.

(2) GR1DV2:

spherically symmetric neutrino radiation hydrodynamics.

(3) SNEC:

the SuperNova explosion code

(4) CCSNMULTIVAR:

multivariate regression analysis of gravitational waves from rotating core collapse.

- AGILE-IDSA

Liebendörfer (2011)

https://physik.unibas.ch/ liebend/download

“... provides tools to run a rudimentary and approximate model of a core-collapse supernova with neutrino transport in spherical symmetry through the phases of stellar collapse, bounce, and early postbounce evolution."

\section{REFERENCES}

Abbott, B. P., et al. (Virgo Collaboration and LIGO Scientific Collaboration), 2016, Phys. Rev. Lett. 116, 061102.

Abrahamyan, S., et al., 2012, Phys. Rev. Lett. 108, 112502.

Ademard, G., et al. (INDRA Collaboration), 2014, Eur. Phys. J. A 50, 33 .

Agnello, M., et al. (FINUDA Collaboration), 2012, Phys. Rev. Lett. 108, 042501.

Agrawal, B., S. Shlomo, and V.K. Au, 2005, Phys. Rev. C 72, 014310.

Agrawal, B. K., J. N. De, and S. K. Samaddar, 2012, Phys. Rev. Lett. 109, 262501.

Agrawal, B. K., J. N. De, S. K. Samaddar, M. Centelles, and X. Viñas, 2014, Eur. Phys. J. A 50, 19.

Ahn, J., et al., 2006, Phys. Lett. B 633, 214.

Aichelin, J., 1991, Phys. Rep. 202, 233.

Aichelin, J., and J. Schaffner-Bielich, 2009, in Relativistic Heavy-Ion Physics Vol. I/23 of Landolt-Börnstein, edited by R. Stock (Springer, Heidelberg).

Akmal, A., V. R. Pandharipande, and D. G. Ravenhall, 1998, Phys. Rev. C 58, 1804.

Alcain, P., P. A. Giménez Molinelli, J. Nichols, and C. Dorso, 2014, Phys. Rev. C 89, 055801.

Alcain, P. N., P. A. Giménez Molinelli, and C. O. Dorso, 2014, Phys. Rev. C 90, 065803.

Alcock, C., E. Farhi, and A. Olinto, 1986, Astrophys. J. 310, 261.

Alexander, G., et al., 1968, Phys. Rev. 173, 1452.

Alford, M., M. Braby, M. Paris, and S. Reddy, 2005, Astrophys. J. 629, 969.

Alford, M., and S. Reddy, 2003, Phys. Rev. D 67, 074024.
Alford, M., et al., 2007, Nature (London) 445, E7.

Alford, M. G., S. Han, and M. Prakash, 2013, Phys. Rev. D 88, 083013.

Alford, M. G., K. Rajagopal, S. Reddy, and A. W. Steiner, 2006, Phys. Rev. D 73, 114016.

Alford, M. G., A. Schmitt, K. Rajagopal, and T. Schäfer, 2008, Rev. Mod. Phys. 80, 1455.

Alkofer, R., and L. von Smekal, 2001, Phys. Rep. 353, 281.

Allton, C., et al., 2002, Phys. Rev. D 66, 074507.

Alm, T., B. L. Friman, G. Röpke, and H. Schulz, 1993, Nucl. Phys. A 551, 45 .

Andronic, A., P. Braun-Munzinger, J. Stachel, and M. Winn, 2012, Phys. Lett. B 718, 80.

Angeli, I., and K. Marinova, 2013, At. Data Nucl. Data Tables 99, 69.

Angeli, I., et al., 2009, J. Phys. G 36, 085102.

Antić, S., and S. Typel, 2015, Nucl. Phys. A 938, 92.

Antoniadis, J., et al., 2013, Science 340, 448.

Aoki, S., et al., 1991, Prog. Theor. Phys. 85, 1287.

Aoki, S., et al., 1995, Phys. Lett. B 355, 45.

Aoki, S., et al. (HAL QCD Collaboration), 2012, Prog. Theor. Exp. Phys. 2012, 01A105.

Arcones, A., H.-T. Janka, and L. Scheck, 2007, Astron. Astrophys. 467, 1227.

Arcones, A., and F.-K. Thielemann, 2013, J. Phys. G 40, 013201.

Arcones, A., et al., 2008, Phys. Rev. C 78, 015806.

Arndt, R. A., W. J. Briscoe, I. I. Strakovsky, and R. L. Workman, 2007, Phys. Rev. C 76, 025209.

Arndt, R. A., I. I. Strakovsky, and R. L. Workman, 1994, Phys. Rev. C 50, 2731.

Audi, G., A. H. Wapstra, and C. Thibault, 2003, Nucl. Phys. A 729, 337.

Avancini, S., C. Barros, D. Menezes, and C. Providência, 2010, Phys. Rev. C 82, 025808

Avancini, S., J. Marinelli, D. Menezes, M. de Moraes, and C. Providência, 2007a, Phys. Rev. C 75, 055805.

Avancini, S., J. Marinelli, D. Menezes, M. de Moraes, and C. Providência, 2007b, Int. J. Mod. Phys. E 16, 2989.

Avancini, S., J. Marinelli, D. Menezes, M. Moraes, and A. Schneider, 2007c, Phys. Rev. C 76, 064318.

Avancini, S., et al., 2008, Phys. Rev. C 78, 015802.

Avancini, S., et al., 2009, Phys. Rev. C 79, 035804.

Avancini, S., et al., 2012, Phys. Rev. C 85, 035806.

Avancini, S. S., S. Chiacchiera, D. P. Menezes, and C. Providência, 2010, Phys. Rev. C 82, 055807.

Aymard, F., F. Gulminelli, and J. Margueron, 2014, Phys. Rev. C 89, 065807.

Baardsen, G., A. Ekström, G. Hagen, and M. Hjorth-Jensen, 2013, Phys. Rev. C 88, 054312.

Baiko, D., and P. Haensel, 1999, Acta Phys. Pol. B 30, 1097.

Baiko, D., A. Potekhin, and D. Yakovlev, 2001, Phys. Rev. E 64, 057402 .

Balberg, S., and A. Gal, 1997, Nucl. Phys. A 625, 435.

Baldo, M., I. Bombaci, and G. F. Burgio, 1997, Astron. Astrophys. 328, 274.

Baldo, M., and F. Burgio, 2001, Lect. Notes Phys. 578, 1.

Baldo, M., and G. Burgio, 2012, Rep. Prog. Phys. 75, 026301.

Baldo, M., G. F. Burgio, M. Centelles, B. K. Sharma, and X. Viñas, 2014, Phys. At. Nucl. 77, 1157.

Baldo, M., L. Ferreira, and O. Nicotra, 2004, Phys. Rev. C 69, 034321.

Baldo, M., and L. S. Ferreira, 1999, Phys. Rev. C 59, 682. 
Baldo, M., A. Fiasconaro, H. Q. Song, G. Giansiracusa, and U. Lombardo, 2001, Phys. Rev. C 65, 017303.

Baldo, M., and C. Maieron, 2004, Phys. Rev. C 69, 014301.

Baldo, M., C. Maieron, P. Schuck, and X. Viñas, 2004, Nucl. Phys. A 736, 241.

Baldo, M., A. Polls, A. Rios, H.-J. Schulze, and I. Vidaña, 2012, Phys. Rev. C 86, 064001.

Baldo, M., L. Robledo, P. Schuck, and X. Viñas, 2013, Phys. Rev. C 87, 064305.

Baldo, M., P. Schuck, and X. Viñas, 2008, Phys. Lett. B 663, 390.

Banik, S., M. Hempel, and D. Bandyopadhyay, 2014, Astrophys. J. Suppl. Ser. 214, 22.

Baran, V., M. Colonna, V. Greco, and M. Di Toro, 2005, Phys. Rep. 410, 335.

Barkat, Z., J.-R. Buchler, and L. Ingber, 1972, Astrophys. J. 176, 723. Barranco, M., and J. R. Buchler, 1980, Phys. Rev. C 22, 1729.

Bart, S., et al., 1999, Phys. Rev. Lett. 83, 5238.

Bartlett, R. J., and M. Musial, 2007, Rev. Mod. Phys. 79, 291.

Bashir, A., et al., 2012, Commun. Theor. Phys. 58, 79.

Bauböck, M., F. Özel, D. Psaltis, and S. M. Morsink, 2015, Astrophys. J. 799, 22.

Bauböck, M., D. Psaltis, and F. Özel, 2013, Astrophys. J. 766, 87.

Baumgarte, T. W., S. A. Teukolsky, S. L. Shapiro, H. T. Janka, and W. Keil, 1996, Astrophys. J. 468, 823.

Bauswein, A., T. Baumgarte, and H. T. Janka, 2013, Phys. Rev. Lett. 111, 131101.

Bauswein, A., S. Goriely, and H. T. Janka, 2013, Astrophys. J. 773, 78.

Bauswein, A., H. Janka, K. Hebeler, and A. Schwenk, 2012, Phys. Rev. D 86, 063001.

Bauswein, A., H.-T. Janka, and R. Oechslin, 2010, Phys. Rev. D 82, 084043.

Bauswein, A., and N. Stergioulas, 2015, Phys. Rev. D 91, 124056.

Bauswein, A., N. Stergioulas, and H.-T. Janka, 2014, Phys. Rev. D 90, 023002 .

Bauswein, A., N. Stergioulas, and H.-T. Janka, 2016, Eur. Phys. J. A 52, 56.

Baym, G., H. A. Bethe, and C. Pethick, 1971, Nucl. Phys. A 175, 225.

Baym, G., and C. Pethick, 1975, Annu. Rev. Nucl. Part. Sci. 25, 27.

Baym, G., and C. Pethick, 1979, Annu. Rev. Astron. Astrophys. 17, 415.

Baym, G., C. Pethick, and P. Sutherland, 1971, Astrophys. J. 170, 299.

Beane, S., W. Detmold, K. Orginos, and M. Savage, 2011, Prog. Part. Nucl. Phys. 66, 1.

Beane, S., et al., 2012, Phys. Rev. Lett. 109, 172001.

Beane, S. R., et al. (NPLQCD Collaboration), 2007, Nucl. Phys. A 794, 62 .

Bedaque, P. F., and G. Rupak, 2003, Phys. Rev. B 67, 174513.

Bedaque, P. F., and U. van Kolck, 2002, Annu. Rev. Nucl. Part. Sci. 52, 339 .

Bednarek, I., P. Haensel, J. Zdunik, M. Bejger, and R. Manka, 2012, Astron. Astrophys. 543, A157.

Beiner, M., H. Flocard, N. van Giai, and P. Quentin, 1975, Nucl. Phys. A 238, 29.

Beisitzer, T., R. Stiele, and J. Schaffner-Bielich, 2014, Phys. Rev. D 90, 085001.

Bender, M., P.-H. Heenen, and P.-G. Reinhard, 2003, Rev. Mod. Phys. 75, 121.

Bender, M., et al., 2009, Phys. Rev. C 80, 064302.

Benic, S., D. Blaschke, D. E. Alvarez-Castillo, T. Fischer, and S. Typel, 2015, Astron. Astrophys. 577, A40.
Bentz, W., and A. W. Thomas, 2001, Nucl. Phys. A 696, 138.

Bertsch, G., and S. Das Gupta, 1988, Phys. Rep. 160, 189.

Beth, E., and G. Uhlenbeck, 1936, Physica (Utrecht) 3, 729.

Beth, E., and G. Uhlenbeck, 1937, Physica (Utrecht) 4, 915.

Bethe, H. A., 1936, Phys. Rev. 50, 332.

Bhattacharyya, A., I. N. Mishustin, and W. Greiner, 2010, J. Phys. G 37, 025201.

Blaschke, D., M. Buballa, A. Radzhabov, and M. Volkov, 2008, Phys. At. Nucl. 71, 1981.

Blaschke, D., H. Grigorian, and D. Voskresensky, 2004, Astron. Astrophys. 424, 979.

Blaschke, D., H. Grigorian, D. Voskresensky, and F. Weber, 2012, Phys. Rev. C 85, 022802.

Blaschke, D., D. Zablocki, M. Buballa, A. Dubinin, and G. Röpke, 2014, Ann. Phys. (Amsterdam) 348, 228.

Blättel, B., V. Koch, and U. Mosel, 1993, Rep. Prog. Phys. 56, 1.

Blinnikov, S., I. Panov, M. Rudzsky, and K. Sumiyoshi, 2011, Astron. Astrophys. 535, A37.

Bodmer, A., Q. Usmani, and J. Carlson, 1984, Phys. Rev. C 29, 684.

Bogdanov, S., 2013, Astrophys. J. 762, 96.

Bogner, S. K., R. J. Furnstahl, and A. Schwenk, 2010, Prog. Part. Nucl. Phys. 65, 94.

Boguta, J., and A. Bodmer, 1977, Nucl. Phys. A 292, 413.

Bombaci, I., A. Fabrocini, A. Polls, and I. Vidaña, 2005, Phys. Lett. B 609, 232.

Bombaci, I., D. Logoteta, C. Providência, and I. Vidaña, 2007, Astron. Astrophys. 462, 1017.

Bonanno, L., and A. Sedrakian, 2012, Astron. Astrophys. 539, A16. Bonche, P., S. Levit, and D. Vautherin, 1985, Nucl. Phys. A 436, 265.

Bonche, P., and D. Vautherin, 1981, Nucl. Phys. A 372, 496.

Bonche, P., and D. Vautherin, 1982, Astron. Astrophys. 112, 268.

Bondorf, J. P., A. S. Botvina, A. S. Iljinov, I. N. Mishustin, and K. Sneppen, 1995, Phys. Rep. 257, 133.

Botvina, A. S., and I. N. Mishustin, 2004, Phys. Lett. B 584, 233.

Botvina, A. S., and I. N. Mishustin, 2010, Nucl. Phys. A 843, 98.

Bowler, R., and M. Birse, 1995, Nucl. Phys. A 582, 655.

Bożek, P., and P. Czerski, 2001, Eur. Phys. J. A 11, 271.

Brack, M., C. Guet, and H.-B. Hakansson, 1985, Phys. Rep. 123, 275.

Brack, M., B. Jennings, and Y. Chu, 1976, Phys. Lett. B 65, 1.

Brack, M., and P. Quentin, 1974a, Phys. Scr. 10A, 163.

Brack, M., and P. Quentin, 1974b, Phys. Lett. B 52, 159.

Brink, D., and E. Boeker, 1967, Nucl. Phys. A 91, 1.

Brito, L., et al., 2006, Phys. Rev. C 74, 045801.

Brockmann, R., and R. Machleidt, 1984, Phys. Lett. B 149, 283.

Brockmann, R., and R. Machleidt, 1990, Phys. Rev. C 42, 1965.

Brown, B. A., 2000, Phys. Rev. Lett. 85, 5296.

Brown, B. A., G. Shen, G. Hillhouse, J. Meng, and A. Trzcinska, 2007, Phys. Rev. C 76, 034305.

Brown, G. E., 1987, Phys. Scr. 36, 209.

Brown, G. E., V. Thorsson, K. Kubodera, and M. Rho, 1992, Phys. Lett. B 291, 355.

Bruenn, S. W., 1985, Astrophys. J. Suppl. Ser. 58, 771.

Brush, S., H. Sahlin, and E. Teller, 1966, J. Chem. Phys. 45, 2102.

Buballa, M., 2005, Phys. Rep. 407, 205.

Buballa, M., and M. Oertel, 1999, Phys. Lett. B 457, 261.

Buballa, M., et al., 2014, J. Phys. G 41, 123001.

Buchler, J.-R., and Z. Barkat, 1971, Phys. Rev. Lett. 27, 48.

Buchler, J.-R., and S. A. Coon, 1977, Astrophys. J. 212, 807.

Burgio, G. F., H.-J. Schulze, and A. Li, 2011, Phys. Rev. C 83, 025804

Burrows, A., 2013, Rev. Mod. Phys. 85, 245.

Burrows, A., and J. M. Lattimer, 1984, Astrophys. J. 285, 294. 
Bürvenich, T., D. Madland, J. Maruhn, and P. Reinhard, 2002, Phys. Rev. C 65, 044308.

Buss, O., et al., 2012, Phys. Rep. 512, 1.

Buyukcizmeci, N., A.S. Botvina, and I. N. Mishustin, 2014, Astrophys. J. 789, 33.

Buyukcizmeci, N., et al., 2013, Nucl. Phys. A 907, 13.

Caballero, O., S. Postnikov, C. Horowitz, and M. Prakash, 2008, Phys. Rev. C 78, 045805.

Cahill, R., J. Praschifka, and C. Burden, 1989, Aust. J. Phys. 42, 161.

Cahill, R., and C. D. Roberts, 1985, Phys. Rev. C 32, 2419.

Caplan, M., A. Schneider, C. Horowitz, and D. Berry, 2014, arXiv:1012.3208.

Carbone, A., 2014, Self-consistent Green's functions with three-body forces, Ph.D. thesis, University of Barcelona, arXiv:1407.6622.

Carbone, A., A. Cipollone, C. Barbieri, A. Rios, and A. Polls, 2013, Phys. Rev. C 88, 054326.

Carbone, A., G. Colo, A. Bracco, L.-G. Cao, P. F. Bortignon, F. Camera, and O. Wieland, 2010, Phys. Rev. C 81, 041301.

Carlson, J., 1987, Phys. Rev. C 36, 2026.

Carlson, J., 1988, Phys. Rev. C 38, 1879.

Carlson, J., S. Gandolfi, and A. Gezerlis, 2012, Prog. Theor. Exp. Phys. 2012, 01A209.

Carlson, J., S. Gandolfi, F. Pederiva, S. C. Pieper, R. Schiavilla, K. E. Schmidt, and R. B. Wiringa, 2015, Rev. Mod. Phys. 87, 1067.

Carlson, J., J. Morales, Jr., V. Pandharipande, and D. Ravenhall, 2003, Phys. Rev. C 68, 025802.

Carlson, J., V. Pandharipande, and R. B. Wiringa, 1983, Nucl. Phys. A 401, 59.

Carnahan, N. F., and K.E. Starling, 1969, J. Chem. Phys. 51, 635.

Carter, B., N. Chamel, and P. Haensel, 2005a, Nucl. Phys. A 759, 441.

Carter, B., N. Chamel, and P. Haensel, 2005b, Nucl. Phys. A 748, 675.

Carter, B., N. Chamel, and P. Haensel, 2006, Int. J. Mod. Phys. D 15, 777.

Centelles, M., X. Roca-Maza, X. Viñas, and M. Warda, 2009, Phys. Rev. Lett. 102, 122502.

Centelles, M., X. Roca-Maza, X. Viñas, and M. Warda, 2010, Phys. Rev. C 82, 054314.

Cerdá-Durán, P., N. DeBrye, M. A. Aloy, J. A. Font, and M. Obergaulinger, 2013, Astrophys. J. 779, L18.

Chabanat, E., P. Bonche, P. Haensel, J. Meyer, and R. Schaeffer, 1998, Nucl. Phys. A 635, 231.

Chabanat, E., J. Meyer, P. Bonche, R. Schaeffer, and P. Haensel, 1997, Nucl. Phys. A 627, 710.

Chabrier, G., F. Douchin, and A. Potekhin, 2002, J. Phys. Condens. Matter 14, 9133.

Chabrier, G., and A. Y. Potekhin, 1998, Phys. Rev. E 58, 4941.

Chajecki, Z., et al., 2014, arXiv:1402.5216.

Chamel, N., 2005, Nucl. Phys. A 747, 109.

Chamel, N., A. Fantina, J. Pearson, and S. Goriely, 2011, Phys. Rev. C 84, 062802.

Chamel, N., and S. Goriely, 2010, Phys. Rev. C 82, 045804.

Chamel, N., and P. Haensel, 2006, Phys. Rev. C 73, 045802.

Chamel, N., and P. Haensel, 2008, Living Rev. Relativ. 11, 10.

Char, P., S. Banik, and D. Bandyopadhyay, 2015, Astrophys. J. 809, 116.

Chatterjee, D., and I. Vidaña, 2016, Eur. Phys. J. A 52, 29.

Chen, H., M. Baldo, G. Burgio, and H.-J. Schulze, 2011, Phys. Rev. D 84, 105023.

Chen, H., M. Baldo, G. F. Burgio, and H.-J. Schulze, 2012, Phys. Rev. D 86, 045006.
Chen, H., J.-B. Wei, M. Baldo, G. Burgio, and H.-J. Schulze, 2015, Phys. Rev. D 91, 105002.

Chen, H., et al., 2008, Phys. Rev. D 78, 116015.

Chen, L.-W., 2011, Phys. Rev. C 83, 044308.

Chen, L.-W., C. M. Ko, and B.-A. Li, 2005a, Phys. Rev. Lett. 94, 032701.

Chen, L.-W., C. M. Ko, and B.-A. Li, 2005b, Phys. Rev. C 72, 064309.

Chen, L.-W., C. M. Ko, B.-A. Li, C. Xu, and J. Xu, 2014, Eur. Phys. J. A 50, 29.

Chen, L.-W., C. M. Ko, B.-A. Li, and J. Xu, 2010, Phys. Rev. C 82, 024321.

Chen, Y., 2012, Eur. Phys. J. A 48, 132.

Chen, Y., 2014, Phys. Rev. C 89, 064306.

Cheng, K., C. Yao, and Z. Dai, 1997, Phys. Rev. C 55, 2092.

Chin, S., and J. Walecka, 1974, Phys. Lett. B 52, 24.

Chodos, A., R. L. Jaffe, K. Johnson, C. B. Thorn, and V. F. Weisskopf, 1974, Phys. Rev. D 9, 3471.

Chomaz, P., F. Gulminelli, C. Ducoin, P. Napolitani, and K. Hasnaoui, 2007, Phys. Rev. C 75, 065805.

Chugunov, A. I., and H. E. DeWitt, 2009, Contrib. Plasma Phys. 49, 696.

Coester, F., 1958, Nucl. Phys. 7, 421.

Coester, F., and H. Kümmel, 1960, Nucl. Phys. 17, 477.

Collins, J. C., and M. J. Perry, 1975, Phys. Rev. Lett. 34, 1353.

Colo, G., U. Garg, and H. Sagawa, 2014, Eur. Phys. J. A 50, 26.

Colucci, G., and A. Sedrakian, 2013, Phys. Rev. C 87, 055806.

Constantinou, C., B. Muccioli, M. Prakash, and J. M. Lattimer, 2014, Phys. Rev. C 89, 065802.

Constantinou, C., B. Muccioli, M. Prakash, and J. M. Lattimer, 2015, Phys. Rev. C 92, 025801.

Contrera, G., A. Grunfeld, and D. Blaschke, 2014, Phys. Part. Nucl. Lett. 11, 342.

Coon, S., and H. Han, 2001, Few-Body Syst. 30, 131.

Coon, S. A., and W. Glöckle, 1981, Phys. Rev. C 23, 1790.

Cooper, E. D., S. Hama, B. C. Clark, and R. L. Mercer, 1993, Phys. Rev. C 47, 297.

Cooper, L. N., 1956, Phys. Rev. 104, 1189.

Cooper, R. L., and L. Bildsten, 2008, Phys. Rev. E 77, 056405.

Cottam, J., F. Paerels, and M. Mendez, 2002, Nature (London) 420, 51.

Cottam, J., F. Paerels, M. Méndez, L. Boirin, W. H. G. Lewin, E. Kuulkers, and J. M. Miller, 2008, Astrophys. J. 672, 504.

Cottingham, W., M. Lacombe, B. Loiseau, J. Richard, and R. Vinh Mau, 1973, Phys. Rev. D 8, 800.

Coupland, D., et al., 2016, Phys. Rev. C 94, 011601.

Cozma, M., 2011, Phys. Lett. B 700, 139.

Cozma, M., Y. Leifels, W. Trautmann, Q. Li, and P. Russotto, 2013, Phys. Rev. C 88, 044912.

Daligault, J., 2006, Phys. Rev. E 73, 056407.

Danielewicz, P., 1984a, Ann. Phys. (N.Y.) 152, 239.

Danielewicz, P., 1984b, Ann. Phys. (N.Y.) 152, 305.

Danielewicz, P., 2003, Nucl. Phys. A 727, 233.

Danielewicz, P., R. Lacey, and W. G. Lynch, 2002, Science 298, 1592.

Danielewicz, P., and J. Lee, 2014, Nucl. Phys. A 922, 1.

Danysz, M., and J. Pniewski, 1953a, Bull. Acad. Pols. Sci. 1, 42.

Danysz, M., and J. Pniewski, 1953b, Philos. Mag. Ser. 5 44, 348.

Daoutidis, I., and S. Goriely, 2011, Phys. Rev. C 84, 027301.

Dasgupta, B., T. Fischer, S. Horiuchi, M. Liebendörfer, A. Mirizzi, I. Sagert, and J. Schaffner-Bielich, 2010, Phys. Rev. D 81, 103005.

Dashen, R., S.-K. Ma, and H. J. Bernstein, 1969, Phys. Rev. 187, 345. 
Davesne, D., et al., 2015, Phys. Rev. C 91, 064303.

Day, B., 1967, Rev. Mod. Phys. 39, 719.

Day, B. D., and J. G. Zabolitzky, 1981, Nucl. Phys. A 366, 221.

Dean, D. J., K. Langanke, and J. M. Sampaio, 2002, Phys. Rev. C 66, 045802.

de Carvalho, S., M. Rotondo, J. A. Rueda, and R. Ruffini, 2014, Phys. Rev. C 89, 015801.

Dechargé, J., and D. Gogny, 1980, Phys. Rev. C 21, 1568.

De Filippo, E., and A. Pagano, 2014, Eur. Phys. J. A 50, 32.

de Forcrand, P., and O. Philipsen, 2002, Nucl. Phys. B 642, 290.

Demorest, P., T. Pennucci, S. Ransom, M. Roberts, and J. Hessels, 2010, Nature (London) 467, 1081.

De Vries, H., C. W. De Jager, and C. De Vries, 1987, At. Data Nucl. Data Tables 36, 495.

DeWitt, H., W. Slattery, and G. Chabrier, 1996, Physica B (Amsterdam) 228, 21.

Dexheimer, V., and S. Schramm, 2008, Astrophys. J. 683, 943.

Dexheimer, V., and S. Schramm, 2010, Phys. Rev. C 81, 045201.

Dickhoff, W., and C. Barbieri, 2004, Prog. Part. Nucl. Phys. 52, 377.

Dieperink, A., Y. Dewulf, D. Van Neck, M. Waroquier, and V. Rodin, 2003, Phys. Rev. C 68, 064307.

Di Toro, M., et al., 2009, Prog. Part. Nucl. Phys. 62, 389.

Đapo, H., B.-J. Schaefer, and J. Wambach, 2008, Eur. Phys. J. A 36, 101.

Đapo, H., B.-J. Schaefer, and J. Wambach, 2010, Phys. Rev. C 81, 035803.

Dobaczewski, J., B. Carlsson, and M. Kortelainen, 2010, J. Phys. G 37, 075106.

Donati, P., P. M. Pizzochero, P. F. Bortignon, and R. A. Broglia, 1994, Phys. Rev. Lett. 72, 2835.

Dong, J., H. Zhang, L. Wang, and W. Zuo, 2013, Phys. Rev. C 88, 014302.

Dong, J., W. Zuo, and J. Gu, 2013, Phys. Rev. C 87, 014303.

Dong, J., W. Zuo, J. Gu, and U. Lombardo, 2012, Phys. Rev. C 85, 034308.

Donoghue, J. F., 2006, Phys. Lett. B 643, 165.

Dorso, C., P. Giménez Molinelli, and J. López, 2012, Phys. Rev. C 86, 055805.

Dorso, C., P. Giménez Molinelli, J. Lopez, and E. Ramirez-Homs, 2012, arXiv:1208.4841.

Dorso, C., P. Giménez Molinelli, J. Nichols, and J. Lopez, 2012, arXiv:1211.5582.

Dorso, C., and J. Randrup, 1993, Phys. Lett. B 301, 328.

Dorso, C. O., P. A. G. Molinelli, and J. A. Lopez, 2011, arXiv:1111.1258.

Douchin, F., and P. Haensel, 2000, Phys. Lett. B 485, 107.

Douchin, F., and P. Haensel, 2001, Astron. Astrophys. 380, 151.

Douchin, F., P. Haensel, and J. Meyer, 2000, Nucl. Phys. A 665, 419.

Downum, C., T. Barnes, J. Stone, and E. Swanson, 2006, Phys. Lett. B 638, 455 .

Drago, A., A. Lavagno, and G. Pagliara, 2014, Phys. Rev. D 89, 043014.

Drago, A., A. Lavagno, G. Pagliara, and D. Pigato, 2014, Phys. Rev. C 90, 065809.

Dreizler, R., and E. Gross, 1990, Density Functional Theory: An Approach to the Quantum Many-Body Problem (Springer, New York).

Drischler, C., V. Soma, and A. Schwenk, 2014, Phys. Rev. C 89, 025806.

Ducoin, C., P. Chomaz, and F. Gulminelli, 2006, Nucl. Phys. A 771, 68.

Ducoin, C., J. Margueron, and P. Chomaz, 2008, Nucl. Phys. A 809, 30 .
Ducoin, C., J. Margueron, C. Providência, and I. Vidaña, 2011, Phys. Rev. C 83, 045810.

Ducoin, C., C. Providência, A. M. Santos, L. Brito, and P. Chomaz, 2008, Phys. Rev. C 78, 055801.

Duflo, J., and A. P. Zuker, 1995, Phys. Rev. C 52, R23.

Dutra, M., et al., 2012, Phys. Rev. C 85, 035201.

Dutra, M., et al., 2014, Phys. Rev. C 90, 055203.

Ebert, D., T. Feldmann, and H. Reinhardt, 1996, Phys. Lett. B 388, 154.

Eisele, F., H. Filthuth, W. Foehlisch, V. Hepp, and G. Zech, 1971, Phys. Lett. B 37, 204.

El Eid, M., and W. Hillebrandt, 1980, Astron. Astrophys. Suppl. Ser. 42, 215.

Ellis, J. R., J. I. Kapusta, and K. A. Olive, 1991, Nucl. Phys. B 348, 345.

Elshamouty, K. G., C. O. Heinke, G. R. Sivakoff, W. C. G. Ho, P. S. Shternin, D. G. Yakovlev, D. J. Patnaude, and L. David, 2013, Astrophys. J. 777, 22.

Endo, T., T. Maruyama, S. Chiba, and T. Tatsumi, 2006, Prog. Theor. Phys. 115, 337.

Engelbrecht, C. A., and J. R. Engelbrecht, 1991, Ann. Phys. (N.Y.) 207, 1.

Engelmann, R., H. Filthuth, V. Hepp, and E. Kluge, 1966, Phys. Lett. 21, 587.

Epelbaum, E., H.-W. Hammer, and U.-G. Meissner, 2009, Rev. Mod. Phys. 81, 1773.

Epelbaum, E., et al., 2014, Phys. Rev. Lett. 112, 102501.

Erler, J., N. Birge, M. Kortelainen, W. Nazarewicz, E. Olsen, A. M. Perhac, and M. Stoitsov, 2012, Nature (London) 486, 509.

Erler, J., C. Horowitz, W. Nazarewicz, M. Rafalski, and P.-G. Reinhard, 2013, Phys. Rev. C 87, 044320.

Erler, J., P. Klüpfel, and P. G. Reinhard, 2010, Phys. Rev. C 82, 044307.

Faber, J. A., and F. A. Rasio, 2012, Living Rev. Relativ. 15, 8.

Fái, G., and J. Randrup, 1982, Nucl. Phys. A 381, 557.

Falanga, M., E. Bozzo, A. Lutovinov, J. M. Bonnet-Bidaud, Y. Fetisova, and J. Puls, 2015, Astron. Astrophys. 577, A130.

Fan, Y.-Z., X.-F. Wu, and D.-M. Wei, 2013, Phys. Rev. D 88, 067304.

Fantina, A., 2015 (private communication).

Fantina, A. F., P. Blottiau, J. Margueron, P. Mellor, and P. M. Pizzochero, 2012, Astron. Astrophys. 541, A30.

Fantina, A. F., N. Chamel, J. M. Pearson, and S. Goriely, 2013, Astron. Astrophys. 559, A128.

Fantoni, S., and A. Fabrocini, 1998, in Microscopic Quantum ManyBody Theories and their Applications, edited by J. Navarro and A. Polls, Vol. 510 of Lecture Notes in Physics (Springer-Verlag, Berlin).

Fantoni, S., and S. Rosati, 1975, Nuovo Cimento Soc. Ital. Fis., A 25, 593.

Farhi, E., and R. Jaffe, 1984, Phys. Rev. D 30, 2379.

Farouki, R., and S. Hamaguchi, 1993, Phys. Rev. E 47, 4330.

Fattoyev, F. J., C. J. Horowitz, J. Piekarewicz, and G. Shen, 2010, Phys. Rev. C 82, 055803.

Fattoyev, F. J., and J. Piekarewicz, 2011, Phys. Rev. C 84, 064302. Fayans, S., 1998, J. Exp. Theor. Phys. Lett. 68, 169.

Fayans, S., S. Tolokonnikov, E. Trykov, and D. Zawischa, 2000, Nucl. Phys. A 676, 49.

Fayans, S., and D. Zawischa, 2001, Int. J. Mod. Phys. B 15, 1684. Fedoseew, A., and H. Lenske, 2015, Phys. Rev. C 91, 034307.

Ferdman, R. D., et al., 2014, Mon. Not. R. Astron. Soc. 443, 2183.

Fetter, A., and J. Walecka, 1971, Quantum theory of many-particle systems (McGraw-Hill, New York). 
M. Oertel et al.: Equations of state for supernovae and compact ...

Feynman, R., N. Metropolis, and E. Teller, 1949, Phys. Rev. 75, 1561.

Finelli, P., N. Kaiser, D. Vretenar, and W. Weise, 2003, Eur. Phys. J. A 17, 573.

Finelli, P., N. Kaiser, D. Vretenar, and W. Weise, 2004, Nucl. Phys. A 735, 449.

Finelli, P., N. Kaiser, D. Vretenar, and W. Weise, 2006, Nucl. Phys. A 770, 1.

Fiolhais, C., F. Nogueira, and M. Marques, 2003, Eds., A Primer in Density Functional Theory, Vol. 620 of Lecture Notes in Physics (Springer, New York).

Fiorilla, S., N. Kaiser, and W. Weise, 2012, Nucl. Phys. A 880, 65.

Fischer, C. S., J. Luecker, and J. A. Mueller, 2011, Phys. Lett. B 702, 438.

Fischer, T., M. Hempel, I. Sagert, Y. Suwa, and J. Schaffner-Bielich, 2014, Eur. Phys. J. A 50, 46.

Fischer, T., T. Klähn, I. Sagert, M. Hempel, and D. Blaschke, 2014, Acta Phys. Pol. B Proc. Suppl. 7, 153.

Fischer, T., I. Sagert, G. Pagliara, M. Hempel, J. Schaffner-Bielich, T. Rauscher, F.-K. Thielemann, R. Käppeli, G. Martínez-Pinedo, and M. Liebendörfer, 2011, Astrophys. J. Suppl. Ser. 194, 39.

Fischer, T., S. C. Whitehouse, A. Mezzacappa, F.-K. Thielemann, and M. Liebendörfer, 2009, Astron. Astrophys. 499, 1.

Fischer, T., S. C. Whitehouse, A. Mezzacappa, F.-K. Thielemann, and M. Liebendörfer, 2010, Astron. Astrophys. 517, A80.

Fischer, T., et al., 2012, Phys. At. Nucl. 75, 613.

Fodor, Z., and S. Katz, 2002, Phys. Lett. B 534, 87.

Fonseca, E., et al., 2016, Astrophys. J. 832, 167.

Fortin, M., J. L. Zdunik, P. Haensel, and M. Bejger, 2015, Astron. Astrophys. 576, A68.

Foucart, F., R. Haas, M. D. Duez, E. O’Connor, C. D. Ott, L. Roberts, L. E. Kidder, J. Lippuner, H. P. Pfeiffer, and M. A. Scheel, 2016, Phys. Rev. D 93, 044019.

Fraga, E. S., R. D. Pisarski, and J. Schaffner-Bielich, 2001, Phys. Rev. D 63, 121702.

Freedman, B. A., and L. D. McLerran, 1977a, Phys. Rev. D 16, 1130. Freedman, B. A., and L. D. McLerran, 1977b, Phys. Rev. D 16, 1147. Freedman, B. A., and L. D. McLerran, 1977c, Phys. Rev. D 16, 1169. Friar, J. L., D. Huber, and U. van Kolck, 1999, Phys. Rev. C 59, 53. Frick, T., 2004, "Self-Consistent Green's Functions in Nuclear Matter at Finite Temperature," Ph.D. thesis, Universität Tübingen. Friedman, B., and V. Pandharipande, 1981, Nucl. Phys. A 361, 502. Friedman, J. L., and N. Stergioulas, 2013, Rotating Relativistic Stars (Cambridge University Press, Cambridge, UK).

Fritsch, S., N. Kaiser, and W. Weise, 2005, Nucl. Phys. A 750, 259.

Fröhlich, C., G. Martínez-Pinedo, M. Liebendörfer, F. K. Thielemann,

E. Bravo, W. R. Hix, K. Langanke, and N. T. Zinner, 2006, Phys. Rev. Lett. 96, 142502.

Fröhlich, C., et al., 2006, Astrophys. J. 637, 415.

Fryer, C. L., K. Belczynski, E. Ramirez-Ruiz, S. Rosswog, G. Shen, and A. W. Steiner, 2015, Astrophys. J. 812, 24.

Fuchs, C., P. Essler, T. Gaitanos, and H. H. Wolter, 1997, Nucl. Phys. A 626, 987.

Fuchs, C., A. Faessler, E. Zabrodin, and Y.-M. Zheng, 2001, Phys. Rev. Lett. 86, 1974.

Fuchs, C., H. Lenske, and H. Wolter, 1995, Phys. Rev. C 52, 3043.

Fuchs, C., and H. Wolter, 2006, Eur. Phys. J. A 30, 5.

Fujita, J., and H. Miyazawa, 1957, Prog. Theor. Phys. 17, 360.

Fukuda, T., et al. (E224 Collaboration), 1998, Phys. Rev. C 58, 1306.

Fukushima, K., 2004, Phys. Lett. B 591, 277.

Fukushima, K., 2008, Phys. Rev. D 77, 114028.

Furnstahl, R., 2002, Nucl. Phys. A 706, 85.

Furnstahl, R., 2004, Lect. Notes Phys. 641, 1.
Furnstahl, R., and K. Hebeler, 2013, Rep. Prog. Phys. 76, 126301. Furnstahl, R., B. D. Serot, and H.-B. Tang, 1997, Nucl. Phys. A 615, 441.

Furusawa, S., H. Nagakura, K. Sumiyoshi, and S. Yamada, 2013, Astrophys. J. 774, 78.

Furusawa, S., K. Sumiyoshi, S. Yamada, and H. Suzuki, 2013, Astrophys. J. 772, 95.

Furusawa, S., S. Yamada, K. Sumiyoshi, and H. Suzuki, 2011, Astrophys. J. 738, 178.

Gaidarov, M., A. Antonov, P. Sarriguren, and E. Moya de Guerra, 2012, Phys. Rev. C 85, 064319.

Gaidarov, M., A. Antonov, P. Sarriguren, and E. Moya de Guerra, 2014, AIP Conf. Proc. 1606, 180.

Gaitanos, T., and M. Kaskulov, 2012, Nucl. Phys. A 878, 49.

Gaitanos, T., and M. Kaskulov, 2015, Nucl. Phys. A 940, 181.

Gaitanos, T., M. Kaskulov, and U. Mosel, 2009, Nucl. Phys. A 828, 9.

Gaitanos, T., and M. M. Kaskulov, 2013, Nucl. Phys. A 899, 133.

Gal, A., E. V. Hungerford, and D. J. Millener, 2016, Rev. Mod. Phys. 88, 035004.

Gale, C., S. Jeon, and B. Schenke, 2013, Int. J. Mod. Phys. A 28, 1340011.

Gale, C., G. M. Welke, M. Prakash, S. J. Lee, and S. Das Gupta, 1990, Phys. Rev. C 41, 1545.

Galloway, D. K., and N. Lampe, 2012, Astrophys. J. 747, 75.

Gandolfi, S., J. Carlson, and S. Reddy, 2012, Phys. Rev. C 85, 032801.

Gandolfi, S., A. Gezerlis, and J. Carlson, 2015, Annu. Rev. Nucl. Part. Sci. 65, 303.

Gandolfi, S., A. Y. Illarionov, K. Schmidt, F. Pederiva, and S. Fantoni, 2009, Phys. Rev. C 79, 054005.

Gearheart, M., W. G. Newton, J. Hooker, and B.-A. Li, 2011, Mon. Not. R. Astron. Soc. 418, 2343.

Geng, L., H. Toki, and J. Meng, 2005, Prog. Theor. Phys. 113, 785.

Gentile, N. A., M. B. Aufderheide, G. J. Mathews, F. D. Swesty, and G. M. Fuller, 1993, Astrophys. J. 414, 701.

Gezerlis, A., et al., 2013, Phys. Rev. Lett. 111, 032501.

Gezerlis, A., et al., 2014, Phys. Rev. C 90, 054323.

Giménez Molinelli, P. A., and C. O. Dorso, 2015, Nucl. Phys. A 933, 306.

Giménez Molinelli, P., J. Nichols, J. López, and C. Dorso, 2014, Nucl. Phys. A 923, 31.

Glendenning, N. K., 1982, Phys. Lett. B 114, 392.

Glendenning, N. K., 1992, Phys. Rev. D 46, 1274.

Glendenning, N. K., 1997, Compact stars: Nuclear physics, particle physics, and general relativity (Springer, New York).

Glendenning, N. K., and S. A. Moszkowski, 1991, Phys. Rev. Lett. 67, 2414.

Gögelein, P., and H. Müther, 2007, Phys. Rev. C 76, 024312.

Gögelein, P., E. van Dalen, C. Fuchs, and H. Müther, 2008, Phys. Rev. C 77, 025802.

Gomes, R. O., V. Dexheimer, S. Schramm, and C. A. Z. Vasconcellos, 2015, Astrophys. J. 808, 8.

Gorenstein, M. I., V. K. Petrov, and G. M. Zinovjev, 1981, Phys. Lett. B 106, 327.

Goriely, S., N. Chamel, and J. Pearson, 2009a, Eur. Phys. J. A 42, 547.

Goriely, S., N. Chamel, and J. Pearson, 2009b, Phys. Rev. Lett. 102, 152503

Goriely, S., N. Chamel, and J. Pearson, 2013a, Phys. Rev. C 88, 024308.

Goriely, S., N. Chamel, and J. Pearson, 2013b, Phys. Rev. C 88, 061302.

Goriely, S., S. Hilaire, and A. J. Koning, 2008, Phys. Rev. C 78, 064307. 
Gourgoulhon, E., P. Grandclément, J.-A. Marck, and J. Novak, and K. Taniguchi, 2016, Astrophysics Source Code Library, record ascl:1608.018.

Greiner, W., and J. Maruhn, 1996, Nuclear Models (Springer-Verlag, Berlin, Heidelberg).

Grill, F., H. Pais, C. Providência, I. Vidaña, and S. S. Avancini, 2014, Phys. Rev. C 90, 045803.

Grill, F., C. Providência, and S. S. Avancini, 2012, Phys. Rev. C 85, 055808.

Gross, D. H. E., 1990, Rep. Prog. Phys. 53, 605.

Gross, D. J., and F. Wilczek, 1973, Phys. Rev. Lett. 30, 1343.

Gross-Boelting, T., C. Fuchs, and A. Faessler, 1999, Nucl. Phys. A 648, 105.

Grossjean, M. K., and H. Feldmeier, 1985, Nucl. Phys. 444, 113.

Guardiola, R., 1998, in Microscopic Quantum Many-Body Theories and their Applications, edited by J. Navarro and A. Polls, Vol. 510 of Lecture Notes in Physics (Springer-Verlag, Berlin).

Guillot, S., and R. E. Rutledge, 2014, Astrophys. J. 796, L3.

Guillot, S., M. Servillat, N. A. Webb, and R. E. Rutledge, 2013, Astrophys. J. 772, 7.

Gulminelli, F., P. Chomaz, A. Raduta, and A. Raduta, 2003, Phys. Rev. Lett. 91, 202701.

Gulminelli, F., A. Raduta, and M. Oertel, 2012, Phys. Rev. C 86, 025805.

Gulminelli, F., A. Raduta, M. Oertel, and J. Margueron, 2013, Phys. Rev. C 87, 055809.

Gulminelli, F., and A. R. Raduta, 2015, Phys. Rev. C 92, 055803.

Gupta, N., and P. Arumugam, 2013, Phys. Rev. C 87, 028801.

Gusakov, M. E., and P. Haensel, 2005, Nucl. Phys. A 761, 333.

Gusakov, M. E., E. M. Kantor, and P. Haensel, 2009a, Phys. Rev. C 79, 055806.

Gusakov, M. E., E. M. Kantor, and P. Haensel, 2009b, Phys. Rev. C 80, 015803.

Gutierrez, E., A. Ahmad, A. Ayala, A. Bashir, and A. Raya, 2014, J. Phys. G 41, 075002.

Güver, T., and F. Özel, 2013, Astrophys. J. 765, L1.

Haensel, P., and B. Pichon, 1994, Astron. Astrophys. 283, 313.

Haensel, P., A. Potekhin, and D. Yakovlev, 2007, Neutron stars 1: Equation of state and structure (Springer, New York).

Haensel, P., and J. Zdunik, 1990, Astron. Astrophys. 229, 117.

Haensel, P., J. Zdunik, M. Bejger, and J. Lattimer, 2009, Astron. Astrophys. 502, 605.

Haensel, P., J. Zdunik, and J. Dobaczewski, 1989, Astron. Astrophys. 222, 353.

Haensel, P., J. Zdunik, and R. Schaeffer, 1986, Astron. Astrophys. 160, 121.

Hagel, K., et al., 2012, Phys. Rev. Lett. 108, 062702.

Hagen, G., M. Hjorth-Jensen, G. R. Jansen, R. Machleidt, and T. Papenbrock, 2012, Phys. Rev. Lett. 109, 032502.

Hagen, G., T. Papenbrock, D. J. Dean, and M. Hjorth-Jensen, 2010, Phys. Rev. C 82, 034330.

Hagen, G., T. Papenbrock, D. J. Dean, A. Schwenk, A. Nogga, M. Wloch, and P. Piecuch, 2007, Phys. Rev. C 76, 034302.

Hagen, G., et al., 2014, Phys. Rev. C 89, 014319.

Haidenbauer, J., and U.-G. Meissner, 2005, Phys. Rev. C 72, 044005.

Haidenbauer, J., U.-G. Meißner, A. Nogga, and H. Polinder, 2007, Lect. Notes Phys. 724, 113.

Haidenbauer, J., et al., 2013, Nucl. Phys. A 915, 24.

Hama, S., B. C. Clark, E. D. Cooper, H. S. Sherif, and R. L. Mercer, 1990, Phys. Rev. C 41, 2737.

Hansen, J. P., 1973, Phys. Rev. A 8, 3096.

Hartle, J. B., and K. S. Thorne, 1968, Astrophys. J. 153, 807.
Hartnack, C., H. Oeschler, and J. Aichelin, 2006, Phys. Rev. Lett. 96, 012302.

Hartnack, C., H. Oeschler, Y. Leifels, E. L. Bratkovskaya, and J. Aichelin, 2012, Phys. Rep. 510, 119.

Hartnack, C., et al., 1998, Eur. Phys. J. A 1, 151.

Hashimoto, M.-a., H. Seki, and M. Yamada, 1984, Prog. Theor. Phys. 71, 320.

Hashimoto, O., and H. Tamura, 2006, Prog. Part. Nucl. Phys. 57, 564.

Hashimoto, T., et al., 2015, Phys. Rev. C 92, 031305.

Hasnaoui, K., and J. Piekarewicz, 2013, Phys. Rev. C 88, 025807.

Hatsuda, T., and T. Kunihiro, 1994, Phys. Rep. 247, 221.

Hebeler, K., J. Lattimer, C. Pethick, and A. Schwenk, 2013, Astrophys. J. 773, 11.

Hebeler, K., and A. Schwenk, 2010, Phys. Rev. C 82, 014314.

Hebeler, K., and A. Schwenk, 2014, Eur. Phys. J. A 50, 11.

Heckel, S., P. P. Schneider, and A. Sedrakian, 2009, Phys. Rev. C 80, 015805 .

Heinke, C., et al., 2014, Mon. Not. R. Astron. Soc. 444, 443.

Heinke, C. O., and W. C. Ho, 2010, Astrophys. J. 719, L167.

Heiselberg, H., and V. Pandharipande, 2000, Annu. Rev. Nucl. Part. Sci. 50, 481.

Heiselberg, H., C. J. Pethick, and D. G. Ravenhall, 1993, Ann. Phys. (N.Y.) 223, 37.

Heiselberg, H., C. J. Pethick, and E. F. Staubo, 1993, Phys. Rev. Lett. 70, 1355.

Hellemans, V., P. Heenen, and M. Bender, 2012, Phys. Rev. C 85, 014326.

Hellemans, V., et al., 2013, Phys. Rev. C 88, 064323.

Hempel, M., 2011, "Equations of State," http://phys-merger.physik .unibas.ch/ hempel/eos.html.

Hempel, M., V. Dexheimer, S. Schramm, and I. Iosilevskiy, 2013, Phys. Rev. C 88, 014906.

Hempel, M., T. Fischer, J. Schaffner-Bielich, and M. Liebendörfer, 2012, Astrophys. J. 748, 70.

Hempel, M., K. Hagel, J. Natowitz, G. Röpke, and S. Typel, 2015, Phys. Rev. C 91, 045805.

Hempel, M., G. Pagliara, and J. Schaffner-Bielich, 2009, Phys. Rev. D 80, 125014.

Hempel, M., and J. Schaffner-Bielich, 2010, Nucl. Phys. A 837, 210.

Hempel, M., J. Schaffner-Bielich, S. Typel, and G. Röpke, 2011, Phys. Rev. C 84, 055804.

Hessels, J. W., et al., 2006, Science 311, 1901.

Hillebrandt, W., K. Nomoto, and R. G. Wolff, 1984, Astron. Astrophys. 133, 175.

Hillebrandt, W., and R. G. Wolff, 1985, "Models of Type II Supernova Explosions," Nucleosynthesis: Challenges and New Developments, edited by W. D. Arnett and J. W. Truran (University of Chicago Press, Chicago), p. 131.

Hix, W. R., O. E. B. Messer, A. Mezzacappa, M. Liebendoerfer, J. Sampaio, K. Langanke, D. J. Dean, and G. Martínez-Pinedo, 2003, Phys. Rev. Lett. 91, 201102.

Ho, T.-L., and E. J. Mueller, 2004, Phys. Rev. Lett. 92, 160404.

Ho, T.-L., and N. Zahariev, 2004, arXiv:cond-mat/0408469.

Hofmann, F., C. Keil, and H. Lenske, 2001a, Phys. Rev. C 64, 025804.

Hofmann, F., C. Keil, and H. Lenske, 2001b, Phys. Rev. C 64, 034314.

Hohenberg, P., and W. Kohn, 1964, Phys. Rev. 136, B864.

Holzenkamp, B., K. Holinde, and J. Speth, 1989, Nucl. Phys. A 500, 485.

Horowitz, C., and D. Berry, 2008, Phys. Rev. C 78, 035806.

Horowitz, C., and D. Berry, 2009, Phys. Rev. C 79, 065803.

Horowitz, C., J. Hughto, A. Schneider, and D. Berry, 2011, arXiv:1109.5095. 
Horowitz, C., M. Perez-Garcia, D. Berry, and J. Piekarewicz, 2005, Phys. Rev. C 72, 035801.

Horowitz, C., and J. Piekarewicz, 2002, Phys. Rev. C 66, 055803.

Horowitz, C., S. Pollock, P. Souder, and R. Michaels, 2001, Phys. Rev. C 63, 025501.

Horowitz, C., and A. Schwenk, 2006a, Nucl. Phys. A 776, 55.

Horowitz, C., and A. Schwenk, 2006b, Phys. Lett. B 642, 326.

Horowitz, C., and A. Schwenk, 2006c, Phys. Lett. B 638, 153.

Horowitz, C., and B. D. Serot, 1987, Nucl. Phys. A 464, 613.

Horowitz, C., et al., 2012, Phys. Rev. C 85, 032501.

Horowitz, C., et al., 2014, J. Phys. G 41, 093001.

Horowitz, C., et al., 2015, Phys. Rev. Lett. 114, 031102.

Horowitz, C. J., D. Berry, and E. Brown, 2007, Phys. Rev. E 75, 066101.

Horowitz, C. J., M. Perez-Garcia, J. Carriere, D. Berry, and J. Piekarewicz, 2004, Phys. Rev. C 70, 065806.

Horowitz, C. J., M. Perez-Garcia, and J. Piekarewicz, 2004, Phys. Rev. C 69, 045804.

Horowitz, C. J., and J. Piekarewicz, 2001a, Phys. Rev. Lett. 86, 5647.

Horowitz, C. J., and J. Piekarewicz, 2001b, Phys. Rev. C 64, 062802 .

Hotokezaka, K., K. Kiuchi, K. Kyutoku, T. Muranushi, Y.-i. Sekiguchi, M. Shibata, and K. Taniguchi, 2013, Phys. Rev. D 88, 044026.

Hotokezaka, K., K. Kyutoku, M. Tanaka, K. Kiuchi, Y. Sekiguchi, M. Shibata, and S. Wanajo, 2013, Astrophys. J. Lett. 778, L16.

Huber, H., F. Weber, and M. Weigel, 1998, Phys. Rev. C 57, 3484.

Hüdepohl, L., B. Müller, H.-T. Janka, A. Marek, and G. G. Raffelt, 2010, Phys. Rev. Lett. 104, 251101.

Hugenholtz, N., and L. van Hove, 1958, Physica (Utrecht) 24, 363.

Huizenga, J. R., and L. G. Moretto, 1972, Annu. Rev. Nucl. Part. Sci. 22, 427.

Huovinen, P., and P. Ruuskanen, 2006, Annu. Rev. Nucl. Part. Sci. 56, 163.

Ichimaru, S., H. Iyetomi, and S. Mitake, 1983, Astrophys. J. 265, L83.

Iliadis, C., 2007, Nuclear Physics of Stars (Wiley-VCH, New York).

Iljinov, A. S., M. V. Mebel, N. Bianchi, E. De Sanctis, C. Guaraldo, V. Lucherini, V. Muccifora, E. Polli, A. R. Reolon, and P. Rossi, 1992, Nucl. Phys. A 543, 517.

Inoue, T., et al. (HAL QCD Collaboration), 2010, Prog. Theor. Phys. 124, 591.

Iosilevskiy, I., 2010, Acta Phys. Polonica B (Proc. Suppl.) 3, 589.

Ishizuka, C., A. Ohnishi, and K. Sumiyoshi, 2003, Nucl. Phys. A 723, 517.

Ishizuka, C., A. Ohnishi, K. Tsubakihara, K. Sumiyoshi, and S. Yamada, 2008, J. Phys. G 35, 085201.

Ishizuka, C., T. Suda, H. Suzuki, A. Ohnishi, K. Sumiyoshi, and H. Toki, 2015, Publ. Astron. Soc. Jpn. 67, 13.

Itoh, N., 1970, Prog. Theor. Phys. 44, 291.

Jaikumar, P., S. Reddy, and A. W. Steiner, 2006, Phys. Rev. Lett. 96, 041101.

Janka, H.-T., 2012a, Annu. Rev. Nucl. Part. Sci. 62, 407.

Janka, H.-T., 2012b, arXiv:1206.2503.

Janka, H.-T., K. Langanke, A. Marek, G. Martínez-Pinedo, and B. Müller, 2007, Phys. Rep. 442, 38.

Jastrzebski, J., et al., 2004, Int. J. Mod. Phys. E 13, 343.

Jin, Z.-P., K. Hotokezaka, X. Li, M. Tanaka, P. D'Avanzo, Y.-Z. Fan, S. Covino, D.-M. Wei, and T. Piran, 2016, Nat. Commun. 7, 12898.

Jog, C. J., and R. A. Smith, 1982, Astrophys. J. 253, 839.

Juodagalvis, A., K. Langanke, W. R. Hix, G. Martínez-Pinedo, and J. M. Sampaio, 2010, Nucl. Phys. A 848, 454.
Kaaret, P., Z. Prieskorn, J. J. M. in 't Zand, S. Brandt, N. Lund, S. Mereghetti, D. Götz, E. Kuulkers, and J. A. Tomsick, 2007, Astrophys. J. Lett. 657, L97.

Kaiser, N., S. Fritsch, and W. Weise, 2002, Nucl. Phys. A 697, 255.

Kaiser, N., S. Fritsch, and W. Weise, 2003, Nucl. Phys. A 724, 47.

Kalantar-Nayestanaki, N., E. Epelbaum, J. Messchendorp, and A. Nogga, 2012, Rep. Prog. Phys. 75, 016301.

Kaplan, D. L., et al., 2013, Astrophys. J. 765, 158.

Kastaun, W., and F. Galeazzi, 2015, Phys. Rev. D 91, 064027.

Katayama, T., and K. Saito, 2014, arXiv:1410.7166.

Kawaguchi, K., K. Kyutoku, M. Shibata, and M. Tanaka, 2016, Astrophys. J. 825, 52.

Keil, W., and H. T. Janka, 1995, Astron. Astrophys. 296, 145.

Khan, E., and J. Margueron, 2013, arXiv:1304.4721.

Khaustov, P., et al. (AGS E885 Collaboration), 2000, Phys. Rev. C 61, 054603.

Kido, T., T. Maruyama, K. Niita, and S. Chiba, 2000, Nucl. Phys. A 663-664, 877c.

Kitaura, F., H.-T. Janka, and W. Hillebrandt, 2006, Astron. Astrophys. 450, 345.

Klähn, T., and T. Fischer, 2015, Astrophys. J. 810, 134.

Klähn, T., D. Blaschke, F. Sandin, C. Fuchs, A. Faessler, H. Grigorian, G. Röpke, and J. Trumper, 2007, Phys. Lett. B 654, 170.

Klähn, T., M. Oertel, and S. Typel, 2013, "CompOSE: Compstar Online Supernova Equations of State," http://compose.obspm.fr.

Klähn, T., C. D. Roberts, L. Chang, H. Chen, and Y.-X. Liu, 2010, Phys. Rev. C 82, 035801.

Klähn, T., et al., 2006, Phys. Rev. C 74, 035802.

Kleinert, H., 1976, Phys. Lett. B 62, 429.

Klevansky, S., 1992, Rev. Mod. Phys. 64, 649.

Klimkiewicz, A., et al., 2007, Phys. Rev. C 76, 051603.

Klos, B., et al., 2007, Phys. Rev. C 76, 014311.

Klüpfel, P., P. G. Reinhard, T. J. Bürvenich, and J. A. Maruhn, 2009, Phys. Rev. C 79, 034310.

Kobyakov, D., and C. Pethick, 2014, Phys. Rev. Lett. 112, 112504.

Köhler, H. S., 1976, Nucl. Phys. A 258, 301.

Kohley, Z., and S. Yennello, 2014, Eur. Phys. J. A 50, 31.

Kohley, Z., et al., 2010, Phys. Rev. C 82, 064601.

Kohn, W., 1999, Rev. Mod. Phys. 71, 1253.

Kohn, W., and L. Sham, 1965, Phys. Rev. 140, A1133.

Kohno, M., Y. Fujiwara, Y. Watanabe, K. Ogata, and M. Kawai, 2006, Phys. Rev. C 74, 064613.

Kojo, T., P. D. Powell, Y. Song, and G. Baym, 2015, Phys. Rev. D 91, 045003.

Kong, H.-Y., Y. Xia, J. Xu, L.-W. Chen, B.-A. Li, and Y.-G. Ma, 2015, Phys. Rev. C 91, 047601.

Kortelainen, M., and T. Lesinski, 2010, J. Phys. G 37, 064039.

Kortelainen, M., et al., 2010, Phys. Rev. C 82, 024313.

Kortelainen, M., et al., 2012, Phys. Rev. C 85, 024304.

Kortelainen, M., et al., 2014, Phys. Rev. C 89, 054314.

Kotake, K., K. Sato, and K. Takahashi, 2006, Rep. Prog. Phys. 69, 971.

Koura, H., T. Tachibana, M. Uno, and M. Yamada, 2005, Prog. Theor. Phys. 113, 305.

Kowalski, S., et al., 2007, Phys. Rev. C 75, 014601.

Krastev, P. G., and F. Sammarruca, 2006, Phys. Rev. C 74, 025808.

Krasznahorkay, A., A. Balanda, J. Bordewijk, S. Brandenburg, M. Harakeh, N. Kalantar-Nayestanaki, B. Nyako, J. Timar, and A. van der Woude, 1994, Nucl. Phys. A 567, 521.

Krasznahorkay, A., et al., 1999, Phys. Rev. Lett. 82, 3216.

Kreim, S., M. Hempel, D. Lunney, and J. Schaffner-Bielich, 2013, Int. J. Mass Spectrom. 349-350, 63. 
Krüger, T., I. Tews, K. Hebeler, and A. Schwenk, 2013, Phys. Rev. C 88, 025802.

Kubis, S., J. Porebska, and D. E. Alvarez-Castillo, 2010, Acta Phys. Pol. B 41, 2449.

Kümmel, H., K. H. Lührmann, and J. G. Zabolitzky, 1978, Phys. Rep. 36, 1.

Kurkela, A., E. S. Fraga, J. Schaffner-Bielich, and A. Vuorinen, 2014, Astrophys. J. 789, 127.

Kurkela, A., P. Romatschke, and A. Vuorinen, 2010, Phys. Rev. D 81, 105021.

Lacombe, M., et al., 1980, Phys. Rev. C 21, 861.

Lacour, A., J. Oller, and U.-G. Meißner, 2011, Ann. Phys. (Amsterdam) 326, 241.

Lagaris, I., and V. Pandharipande, 1981, Nucl. Phys. A 359, 331.

Lähde, T. A., et al., 2014, Phys. Lett. B 732, 110.

Lalazissis, G., S. Karatzikos, M. Serra, T. Otsuka, and P. Ring, 2009, Phys. Rev. C 80, 041301.

Lalazissis, G. A., J. König, and P. Ring, 1997, Phys. Rev. C 55, 540.

Lalazissis, G. A., S. Raman, and P. Ring, 1999, At. Data Nucl. Data Tables 71, 1

Lamb, D., J. Lattimer, C. Pethick, and D. Ravenhall, 1978, Phys. Rev. Lett. 41, 1623.

Lamb, D., J. Lattimer, C. Pethick, and D. Ravenhall, 1983, Nucl. Phys. A 411, 449.

Landau, L. D., and E. Lifshitz, 1980, Statistical Physics: Part 1 (Butterworth-Heinemann, Oxford).

Langanke, K., G. Martínez-Pinedo, J. M. Sampaio, D. J. Dean, W. R. Hix, O. E. B. Messer, A. Mezzacappa, M. Liebendörfer, H. T. Janka, and M. Rampp, 2003, Phys. Rev. Lett. 90, 241102.

Lattimer, J., 1981, Annu. Rev. Nucl. Part. Sci. 31, 337.

Lattimer, J., C. Pethick, D. Ravenhall, and D. Lamb, 1985, Nucl. Phys. A 432, 646.

Lattimer, J., and M. Prakash, 2001, Astrophys. J. 550, 426.

Lattimer, J., and M. Prakash, 2004, Science 304, 536.

Lattimer, J. M., 2012, Annu. Rev. Nucl. Part. Sci. 62, 485.

Lattimer, J. M., A. Burrows, and A. Yahil, 1985, Astrophys. J. 288, 644.

Lattimer, J. M., and Y. Lim, 2013, Astrophys. J. 771, 51.

Lattimer, J. M., C. J. Pethick, D. G. Ravenhall, and D. Q. Lamb, 1985, Nucl. Phys. A 432, 646.

Lattimer, J. M., and M. Prakash, 2000, Phys. Rep. 333-334, 121.

Lattimer, J. M., and M. Prakash, 2007, Phys. Rep. 442, 109.

Lattimer, J. M., and M. Prakash, 2011, in From Nuclei to Stars (World Scientific, Singapore), pp. 275-304.

Lattimer, J. M., and D. N. Schramm, 1974, Astrophys. J. 192, L145. Lattimer, J. M., and A. W. Steiner, 2014, Eur. Phys. J. A 50, 40.

Lattimer, J. M., and F. D. Swesty, 1991, Nucl. Phys. A 535, 331.

Lattimer, J. M., and F. D. Swesty, 1991, "Lattimer-Swesty EOS Web Site," http://www.astro.sunysb.edu/dswesty/lseos.html.

Lawrence, S., J. G. Tervala, P. F. Bedaque, and M. C. Miller, 2015, Astrophys. J. 808, 186.

Leahy, D. A., S. M. Morsink, and Y. Chou, 2011, Astrophys. J. 742, 17

Lee, D., 2009, Prog. Part. Nucl. Phys. 63, 117.

Le Fèvre, A., Y. Leifels, W. Reisdorf, J. Aichelin, and C. Hartnack, 2016, Nucl. Phys. A 945, 112.

Lejeune, A., U. Lombardo, and W. Zuo, 2000, Phys. Lett. B 477, 45. Lesinski, T., M. Bender, K. Bennaceur, T. Duguet, and J. Meyer, 2007, Phys. Rev. C 76, 014312.

Li, B.-A., 2002, Nucl. Phys. A 708, 365.

Li, B.-A., and L.-W. Chen, 2005, Phys. Rev. C 72, 064611.

Li, B.-A., L.-W. Chen, and C. M. Ko, 2008, Phys. Rep. 464, 113.
Li, B.-A., and X. Han, 2013, Phys. Lett. B 727, 276.

Li, B.-A., A. Ramos, G. Verde, and Vidaña, I., 2014, Eds., Topical Issue on Nuclear Symmetry Energy, Vol. A50 of Eur. Phys. J. (Springer, New York).

Liebendörfer, M., 2011, “AGILE-IDSA Web Site,” https://physik .unibas.ch/ liebend/download.

Lin, J., F. Özel, D. Chakrabarty, and D. Psaltis, 2010, Astrophys. J. 723, 1053.

Lipparini, E., and S. Stringari, 1989, Phys. Rep. 175, 103.

Liu, M., N. Wang, Z.-X. Li, and F.-S. Zhang, 2010, Phys. Rev. C 82, 064306.

Liu, M.-Q., J. Zhang, and Z.-Q. Luo, 2007, Chin. Phys. 16, 3146.

Liu, X.-J., H. Hu, and P. D. Drummond, 2009, Phys. Rev. Lett. 102, 160401.

Lombardo, U., and H. Schulze, 2001, Lect. Notes Phys. 578, 30.

Lonardoni, D., S. Gandolfi, and F. Pederiva, 2013, Phys. Rev. C 87, 041303.

Lonardoni, D., A. Lovato, S. Gandolfi, and F. Pederiva, 2015, Phys. Rev. Lett. 114, 092301

Lonardoni, D., F. Pederiva, and S. Gandolfi, 2014, Phys. Rev. C 89, 014314.

Long, W., N. Van Giai, and J. Meng, 2006, Phys. Lett. B 640, 150.

Long, W. H., P. Ring, N. Van Giai, and J. Meng, 2010, Phys. Rev. C 81, 024308.

Long, W. H., H. Sagawa, N. Van Giai, and J. Meng, 2007, Phys. Rev. C 76, 034314.

Long, W.-h., J. Meng, N. Van Giai, and S.-G. Zhou, 2004, Phys. Rev. C 69, 034319.

Lopes, L. L., and D. P. Menezes, 2014, Phys. Rev. C 89, 025805.

Lorenz, C., D. Ravenhall, and C. Pethick, 1993, Phys. Rev. Lett. 70, 379.

Machleidt, R., 2001, Phys. Rev. C 63, 024001.

Machleidt, R., and D. Entem, 2011, Phys. Rep. 503, 1.

Machleidt, R., K. Holinde, and C. Elster, 1987, Phys. Rep. 149, 1.

Machleidt, R., and G.-Q. Li, 1994, Phys. Rep. 242, 5.

Maessen, P. M. M., T. A. Rijken, and J. J. de Swart, 1989, Phys. Rev. C 40, 2226.

Magierski, P., A. Bulgac, and P.-H. Heenen, 2003, Nucl. Phys. A 719, C217.

Magierski, P., and P.-H. Heenen, 2002, Phys. Rev. C 65, 045804.

Mallik, S., J. De, S. Samaddar, and S. Sarkar, 2008, Phys. Rev. C 77, 032201.

Marek, A., and H.-T. Janka, 2009, Astrophys. J. 694, 664.

Marek, A., H.-T. Janka, and E. Müller, 2009, Astron. Astrophys. 496, 475.

Margueron, J., S. Goriely, M. Grasso, G. Colo, and H. Sagawa, 2009, J. Phys. G 36, 125103.

Margueron, J., S. Goriely, M. Grasso, G. Colo, and H. Sagawa, 2010, Mod. Phys. Lett. A 25, 1771.

Margueron, J., M. Grasso, S. Goriely, G. Colo, and H. Sagawa, 2012, Prog. Theor. Phys. Suppl. 196, 172.

Margueron, J., J. Navarro, and P. Blottiau, 2004, Phys. Rev. C 70, 028801 .

Margueron, J., and H. Sagawa, 2009, J. Phys. G 36, 125102.

Martin, N., and M. Urban, 2015, Phys. Rev. C 92, 015803.

Martínez-Pinedo, G., T. Fischer, A. Lohs, and L. Huther, 2012, Phys. Rev. Lett. 109, 251104.

Martínez-Pinedo, G., T. Fischer, and L. Hüther, 2014, J. Phys. G 41, 044008 .

Maruyama, T., S. Chiba, H.-J. Schulze, and T. Tatsumi, 2008, Phys. Lett. B 659, 192.

Maruyama, T., T. Tatsumi, D. N. Voskresensky, T. Tanigawa, and S. Chiba, 2005, Phys. Rev. C 72, 015802. 
Maruyama, T., G. Watanabe, and S. Chiba, 2012, Prog. Theor. Exp. Phys. 2012, 01A201.

Maruyama, T., et al., 1998, Phys. Rev. C 57, 655.

Masuda, K., T. Hatsuda, and T. Takatsuka, 2013, Prog. Theor. Exp. Phys. 2013, 073D01.

Masuda, K., T. Hatsuda, and T. Takatsuka, 2016, Prog. Theor. Exp. Phys. 2016, 021D01.

Matsuzaki, M., 2006, Phys. Rev. C 73, 028801.

Mazurek, T. J., J. M. Lattimer, and G. E. Brown, 1979, Astrophys. J. 229, 713.

Meißner, U. G., J. A. Oller, and A. Wirzba, 2002, Ann. Phys. (Amsterdam) 297, 27.

Menezes, D. P., and C. Providência, 2007, arXiv:astro-ph/0703649.

Meng, J., et al., 2006, Prog. Part. Nucl. Phys. 57, 470.

Mezzacappa, A., 2005, Annu. Rev. Nucl. Part. Sci. 55, 467.

Migdal, A. B., E. E. Saperstein, M. A. Troitsky, and D. N. Voskresensky, 1990, Phys. Rep. 192, 179.

Miller, M. C., 2013, arXiv:1312.0029.

Mishustin, I., L. Satarov, H. Stoecker, and W. Greiner, 2002, Phys. Rev. C 66, 015202.

Miyatsu, T., S. Yamamuro, and K. Nakazato, 2013, Astrophys. J. 777, 4.

Möller, P., W. D. Myers, H. Sagawa, and S. Yoshida, 2012, Phys. Rev. Lett. 108, 052501.

Möller, P., J. R. Nix, W. D. Myers, and W. J. Swiatecki, 1995, At. Data Nucl. Data Tables 59, 185.

Moustakidis, C.C., and C.P. Panos, 2009, Phys. Rev. C 79, 045806.

Mukhopadhyay, T., and D. N. Basu, 2007, Acta Phys. Pol. B 38, 3225.

Mulero, Á., 2008, Ed., Theory and Simulation of Hard-Sphere Fluids and Related Systems, Vol. 753 of Lecture Notes in Physics (Springer-Verlag, Berlin).

Müller, D., M. Buballa, and J. Wambach, 2013, Eur. Phys. J. A 49, 96.

Müller, H., and B. D. Serot, 1995, Phys. Rev. C 52, 2072.

Müller, H., and B. D. Serot, 1996, Nucl. Phys. A 606, 508.

Müther, H., and A. Polls, 2000, Prog. Part. Nucl. Phys. 45, 243.

Myers, W. D., and W. J. Swiatecki, 1990, Ann. Phys. (N.Y.) 204, 401.

Myers, W. D., and W. J. Swiatecki, 1994, Lawrence Berkeley Laboratory Report No. LBL-36803.

Myers, W. D., and W. J. Swiatecki, 1996, Nucl. Phys. A 601, 141. Nadyozhin, D. K., and A. V. Yudin, 2004, Astron. Lett. 30, 634.

Nadyozhin, D. K., and A. V. Yudin, 2005, Astron. Lett. 31, 271.

Nagae, T., et al., 1998, Phys. Rev. Lett. 80, 1605.

Nagels, M., T. Rijken, and J. de Swart, 1977, Phys. Rev. D 15, 2547.

Nagels, M., T. Rijken, and J. de Swart, 1978, Phys. Rev. D 17, 768.

Nagels, M., T. A. Rijken, and Y. Yamamoto, 2014, arXiv:1408.4825.

Nakada, H., 2003, Phys. Rev. C 68, 014316.

Nakazato, K., S. Furusawa, K. Sumiyoshi, A. Ohnishi, S. Yamada, and H. Suzuki, 2012, Astrophys. J. 745, 197.

Nakazato, K., K. Iida, and K. Oyamatsu, 2011, Phys. Rev. C 83, 065811 .

Nakazato, K., K. Oyamatsu, and S. Yamada, 2009, Phys. Rev. Lett. 103, 132501.

Nakazato, K., K. Sumiyoshi, H. Suzuki, and S. Yamada, 2010, Phys. Rev. D 81, 083009.

Nakazato, K., K. Sumiyoshi, and S. Yamada, 2008, Phys. Rev. D 77, 103006.

Nakazato, K., K. Sumiyoshi, and S. Yamada, 2010, Astrophys. J. 721, 1284.

Nakazato, K., K. Sumiyoshi, and S. Yamada, 2013, Astron. Astrophys. 558, A50.
Nakazawa, K. (KEK-E176 and J-PARC-E07 Collaborations), 2010, Nucl. Phys. A 835, 207.

Nambu, Y., and G. Jona-Lasinio, 1961a, Phys. Rev. 122, 345.

Nambu, Y., and G. Jona-Lasinio, 1961b, Phys. Rev. 124, 246.

Napolitani, P., P. Chomaz, F. Gulminelli, and K. Hasnaoui, 2007, Phys. Rev. Lett. 98, 131102.

Natowitz, J., et al., 2010, Phys. Rev. Lett. 104, 202501.

Navarro, J., and A. Polls, 2013, Phys. Rev. C 87, 044329.

Navarro Pérez, R., J. E. Amaro, and E. R. Arriola, 2015, Phys. Rev. C 91, 054002.

Navarro Pérez, R., J. E. Amaro, and E. Ruiz Arriola, 2013, Phys. Rev. C 88, 024002 [88, 069902(E) (2013)].

Nazarewicz, W., P. G. Reinhard, W. Satula, and D. Vretenar, 2014, Eur. Phys. J. A 50, 20.

Negele, J. W., and D. Vautherin, 1972, Phys. Rev. C 5, 1472.

Negele, J. W., and D. Vautherin, 1973, Nucl. Phys. A 207, 298.

Newton, W., and J. Stone, 2009, Phys. Rev. C 79, 055801.

Newton, W. G., and B.-a. Li, 2009, Phys. Rev. C 80, 065809.

Nickel, D., R. Alkofer, and J. Wambach, 2006, Phys. Rev. D 74, 114015 .

Nickel, D., J. Wambach, and R. Alkofer, 2006, Phys. Rev. D 73, 114028.

Nicotra, O. E., M. Baldo, G. Burgio, and H.-J. Schulze, 2006a, Astron. Astrophys. 451, 213.

Nicotra, O. E., M. Baldo, G. F. Burgio, and H.-J. Schulze, 2006b, Phys. Rev. D 74, 123001.

Nikolaus, B., T. Hoch, and D. Madland, 1992, Phys. Rev. C 46, 1757.

Nikšić, T., T. Marketin, D. Vretenar, N. Paar, and P. Ring, 2005, Phys. Rev. C 71, 014308.

Nikšić, T., D. Vretenar, P. Finelli, and P. Ring, 2002, Phys. Rev. C 66, 024306.

Nikšić, T., D. Vretenar, and P. Ring, 2011, Prog. Part. Nucl. Phys. 66, 519.

Nishimura, N., et al., 2012, Astrophys. J. 758, 9.

Nozawa, T., N. Stergioulas, E. Gourgoulhon, and Y. Eriguchi, 1998, Astron. Astrophys. Suppl. Ser. 132, 431.

O'Connor, E., D. Gazit, C. Horowitz, A. Schwenk, and N. Barnea, 2007, Phys. Rev. C 75, 055803.

O'Connor, E., and C. D. Ott, 2008, http://www.stellarcollapse.org.

O'Connor, E., and C. D. Ott, 2011, Astrophys. J. 730, 70.

O'Connor, E., and C. D. Ott, 2013, Astrophys. J. 762, 126.

Oechslin, R., H.-T. Janka, and A. Marek, 2007, Astron. Astrophys. 467, 395.

Oertel, M., A. Fantina, and J. Novak, 2012, Phys. Rev. C 85, 055806.

Oertel, M., F. Gulminelli, C. Providência, and A. R. Raduta, 2016, Eur. Phys. J. A 52, 50.

Oertel, M., C. Providência, F. Gulminelli, and A. R. Raduta, 2015, J. Phys. G 42, 075202.

Oertel, M., and M. Urban, 2008, Phys. Rev. D 77, 074015.

Ogata, S., H. Iyetomi, S. Ichimaru, and H. M. Van Horn, 1993, Phys. Rev. E 48, 1344.

Okamoto, M., T. Maruyama, K. Yabana, and T. Tatsumi, 2012, Phys. Lett. B 713, 284.

Oller, J., A. Lacour, and U.-G. Meißner, 2010, J. Phys. G 37, 015106.

Ono, A., H. Horiuchi, T. Maruyama, and A. Ohnishi, 1992, Prog. Theor. Phys. 87, 1185.

Onsi, M., et al., 2008, Phys. Rev. C 77, 065805.

Oppenheimer, J. R., and G. M. Volkoff, 1939, Phys. Rev. 55, 374.

Ott, C. D., 2009, Classical Quantum Gravity 26, 063001.

Oyamatsu, K., 1993, Nucl. Phys. A 561, 431.

Oyamatsu, K., 1994, Nucl. Phys. A 578, 181. 
Oyamatsu, K., M.-a. Hashimoto, and M. Yamada, 1984, Prog. Theor. Phys. 72, 373.

Oyamatsu, K., and K. Iida, 2007, Phys. Rev. C 75, 015801.

Özel, F., G. Baym, and T. Güver, 2010, Phys. Rev. D 82, 101301.

Özel, F., A. Gould, and T. Güver, 2012, Astrophys. J. 748, 5.

Özel, F., and D. Psaltis, 2009, Phys. Rev. D 80, 103003.

Özel, F., D. Psaltis, T. Güver, G. Baym, C. Heinke, and S. Guillot, 2016, Astrophys. J. 820, 28.

Paar, N., C. C. Moustakidis, T. Marketin, D. Vretenar, and G. Lalazissis, 2014, Phys. Rev. C 90, 011304.

Page, D., U. Geppert, and F. Weber, 2006, Nucl. Phys. A 777, 497. Page, D., J. M. Lattimer, M. Prakash, and A. W. Steiner, 2014, Novel Superfluids: Volume 2, International Series of Monographs on Physics (Oxford University Press, New York), Chap. 21, pp. 505-579.

Page, D., M. Prakash, J. M. Lattimer, and A. W. Steiner, 2011, Phys. Rev. Lett. 106, 081101.

Pais, A., and G. Uhlenbeck, 1959, Phys. Rev. 116, 250.

Pais, H., S. Chiacchiera, and C. Providência, 2015, Phys. Rev. C 91, 055801.

Pais, H., W. G. Newton, and J. R. Stone, 2014, Phys. Rev. C 90, 065802.

Pais, H., and J. R. Stone, 2012, Phys. Rev. Lett. 109, 151101.

Pandharipande, V., and R. B. Wiringa, 1979, Rev. Mod. Phys. 51, 821.

Pannarale, F., L. Rezzolla, F. Ohme, and J. S. Read, 2011, Phys. Rev. D 84, 104017.

Papakonstantinou, P., J. Margueron, F. Gulminelli, and A. R. Raduta, 2013, Phys. Rev. C 88, 045805.

Pastore, A., et al., 2014, Phys. Rev. C 90, 025804.

Pavón Valderrama, M., and D. R. Phillips, 2015, Phys. Rev. Lett. 114, 082502.

Pearson, J., N. Chamel, S. Goriely, and C. Ducoin, 2012, Phys. Rev. C 85, 065803.

Pearson, J., N. Chamel, A. Pastore, and S. Goriely, 2015, Phys. Rev. C 91, 018801.

Pearson, J., S. Goriely, and N. Chamel, 2011, Phys. Rev. C 83, 065810.

Perego, A., M. Hempel, C. Fröhlich, K. Ebinger, M. Eichler, J. Casanova, M. Liebendörfer, and F.-K. Thielemann, 2015, Astrophys. J. 806, 275.

Perego, A., S. Rosswog, R. M. Cabezón, O. Korobkin, R. Käppeli, A. Arcones, and M. Liebendörfer, 2014, Mon. Not. R. Astron. Soc. 443, 3134.

Peres, B., M. Oertel, and J. Novak, 2013, Phys. Rev. D 87, 043006.

Pethick, C., D. Ravenhall, and C. Lorenz, 1995, Nucl. Phys. A 584, 675.

Pethick, C., and V. Thorsson, 1997, Phys. Rev. D 56, 7548.

Pethick, C. J., and D. G. Ravenhall, 1988, Ann. Phys. (N.Y.) 183, 131.

Piarulli, M., L. Girlanda, R. Schiavilla, R. Navarro Pérez, J.E. Amaro, and E. Ruiz Arriola, 2015, Phys. Rev. C 91, 024003.

Piekarewicz, J., 2004, Phys. Rev. C 69, 041301.

Piekarewicz, J., 2010, J. Phys. G 37, 064038.

Piekarewicz, J., B. K. Agrawal, G. Colò, W. Nazarewicz, N. Paar, P.-G. Reinhard, X. Roca-Maza, and D. Vretenar, 2012, Phys. Rev. C 85, 041302.

Piekarewicz, J., and G. Toledo Sanchez, 2012, Phys. Rev. C 85, 015807.

Pieper, S. C., 2008, Riv. Nuovo Cimento 31, 709.

Pieper, S. C., V. Pandharipande, R. B. Wiringa, and J. Carlson, 2001, Phys. Rev. C 64, 014001.

Podsiadlowski, P., et al., 2005, Mon. Not. R. Astron. Soc. 361, 1243.
Polinder, H., J. Haidenbauer, and U.-G. Meißner, 2006, Nucl. Phys. A 779, 244.

Politzer, H. D., 1974, Phys. Rep. 14, 129.

Pollock, E., and J. Hansen, 1973, Phys. Rev. A 8, 3110.

Pons, J. A., J. A. Miralles, M. Prakash, and J. M. Lattimer, 2001, Astrophys. J. 553, 382.

Pons, J. A., S. Reddy, P. J. Ellis, M. Prakash, and J. M. Lattimer, 2000, Phys. Rev. C 62, 035803.

Pons, J. A., S. Reddy, M. Prakash, J. M. Lattimer, and J. A. Miralles, 1999, Astrophys. J. 513, 780.

Pons, J. A., A. W. Steiner, M. Prakash, and J. M. Lattimer, 2001, Phys. Rev. Lett. 86, 5223.

Pons, J. A., D. Viganò, and N. Rea, 2013, Nat. Phys. 9, 431.

Posselt, B., G. Pavlov, V. Suleimanov, and O. Kargaltsev, 2013, Astrophys. J. 779, 186.

Potekhin, A., 2014, Phys. Usp. 57, 735.

Potekhin, A., A. Fantina, N. Chamel, J. Pearson, and S. Goriely, 2013, Astron. Astrophys. 560, A48.

Potekhin, A., and P. Haensel, 2013, "Equations of State," http://www ioffe.ru/astro/NSG/nseoslist.html.

Potekhin, A. Y., 2010, Phys. Usp. 53, 1235, http://stacks.iop.org/ 1063-7869/53/i=12/a=R03.

Potekhin, A. Y., and G. Chabrier, 2000, Phys. Rev. E 62, 8554.

Potekhin, A. Y., and G. Chabrier, 2010, Contrib. Plasma Phys. 50, 82.

Potekhin, A. Y., G. Chabrier, A. I. Chugunov, H. E. DeWitt, and F. J. Rogers, 2009, Phys. Rev. E 80, 047401.

Potekhin, A. Y., G. Chabrier, and F. J. Rogers, 2009, Phys. Rev. E 79, 016411.

Potekhin, A. Y., J. A. Pons, and D. Page, 2015, Space Sci. Rev. 191, 239.

Poutanen, J., et al., 2014, Mon. Not. R. Astron. Soc. 442, 3777.

Prakash, M., J. R. Cooke, and J. M. Lattimer, 1995, Phys. Rev. D 52, 661.

Prakash, M., P. J. Ellis, and J. I. Kapusta, 1992, Phys. Rev. C 45, 2518.

Prakash, M., et al., 1997, Phys. Rep. 280, 1.

Pratt, S., P. Siemens, and Q. Usmani, 1987, Phys. Lett. B 189, 1.

Providência, C., L. Brito, S. Avancini, D. Menezes, and P. Chomaz, 2006, Phys. Rev. C 73, 025805.

Providência, C., L. Brito, A. Santos, D. Menezes, and S. Avancini, 2006, Phys. Rev. C 74, 045802.

Pudliner, B., V. Pandharipande, J. Carlson, S. C. Pieper, and R. B. Wiringa, 1997, Phys. Rev. C 56, 1720.

Pudliner, B., V. Pandharipande, J. Carlson, and R. B. Wiringa, 1995, Phys. Rev. Lett. 74, 4396.

Qin, L., et al., 2012, Phys. Rev. Lett. 108, 172701.

Qin, S.-x., L. Chang, H. Chen, Y.-x. Liu, and C. D. Roberts, 2011, Phys. Rev. Lett. 106, 172301.

Quentin, P., and H. Flocard, 1978, Annu. Rev. Nucl. Part. Sci. 28, 523.

Raduta, A., F. Aymard, and F. Gulminelli, 2014, Eur. Phys. J. A 50, 24.

Raduta, A., and F. Gulminelli, 2009, Phys. Rev. C 80, 024606.

Raduta, A., and F. Gulminelli, 2010, Phys. Rev. C 82, 065801.

Raduta, A. R., F. Gulminelli, and M. Oertel, 2016, Phys. Rev. C 93, 025803.

Ratti, C., M. A. Thaler, and W. Weise, 2006, Phys. Rev. D 73, 014019.

Rauscher, T., 2003, Astrophys. J. Suppl. Ser. 147, 403.

Rauscher, T., and F.-K. Thielemann, 2000, At. Data Nucl. Data Tables 75, 1 .

Rauscher, T., and F.-K. Thielemann, 2001, At. Data Nucl. Data Tables 79, 47. 
Ravenhall, D., C. Bennett, and C. Pethick, 1972, Phys. Rev. Lett. 28, 978.

Ravenhall, D., C. Pethick, and J. Wilson, 1983, Phys. Rev. Lett. 50, 2066.

Ray, L., 1979, Phys. Rev. C 19, 1855.

Ray, L., and P. Hodgson, 1979, Phys. Rev. C 20, 2403.

Read, J. S., B. D. Lackey, B. J. Owen, and J. L. Friedman, 2009, Phys. Rev. D 79, 124032.

Read, J. S., et al., 2013, Phys. Rev. D 88, 044042.

Reinhard, P., 1989, Rep. Prog. Phys. 52, 439.

Reinhard, P., M. Rufa, J. Maruhn, W. Greiner, and J. Friedrich, 1986, Z. Phys. A 323, 13.

Reinhard, P.-G., and W. Nazarewicz, 2010, Phys. Rev. C 81, 051303.

Reinhard, P.-G., and W. Nazarewicz, 2013, Phys. Rev. C 87, 014324.

Reisdorf, W., et al. (FOPI Collaboration), 2007, Nucl. Phys. A 781, 459.

Reisdorf, W., et al. (FOPI Collaboration), 2012, Nucl. Phys. A 876, 1.

Rijken, T., 2006, Phys. Rev. C 73, 044007.

Rijken, T., and Y. Yamamoto, 2006, Phys. Rev. C 73, 044008.

Rijken, T. A., V. G. J. Stoks, and Y. Yamamoto, 1999, Phys. Rev. C 59, 21.

Rikovska Stone, J., J. C. Miller, R. Koncewicz, P. D. Stevenson, and M. R. Strayer, 2003, Phys. Rev. C 68, 034324.

Rikovska Stone, J., P. D. Stevenson, J. C. Miller, and M. R. Strayer, 2002, Phys. Rev. C 65, 064312.

Rikovska-Stone, J., P. A. Guichon, H. H. Matevosyan, and A. W. Thomas, 2007, Nucl. Phys. A 792, 341.

Ring, P., 1996, Prog. Part. Nucl. Phys. 37, 193.

Ring, P., and P. Schuck, 1980, The nuclear many-body problem (Springer-Verlag, Berlin/Heidelberg).

Rios, A., A. Polls, A. Ramos, and H. Müther, 2008, Phys. Rev. C 78, 044314.

Rios, A., A. Polls, and I. Vidaña, 2009, Phys. Rev. C 79, 025802.

Rischke, D. H., M. I. Gorenstein, H. Stoecker, and W. Greiner, 1991, Z. Phys. C 51, 485.

Roberts, C. D., 2015, "Strong QCD and Dyson-Schwinger equations" in EMS IRMA Lectures in Mathematics and Theoretical Physics, edited by K. Ebrahimi-Fard and F. Fauvet (European Mathematical Society Publishing House, Zürich, Switzerland), Vol. 21, p. 355.

Roberts, C. D., R. Cahill, and J. Praschifka, 1988, Ann. Phys. (N.Y.) 188, 20.

Roberts, C. D., and S. M. Schmidt, 2000, Prog. Part. Nucl. Phys. 45, S1.

Roberts, L., S. Reddy, and G. Shen, 2012, Phys. Rev. C 86, 065803.

Roberts, L., et al., 2012, Phys. Rev. Lett. 108, 061103.

Roberts, L. F., 2012, Astrophys. J. 755, 126.

Roberts, L. F., S. E. Woosley, and R. D. Hoffman, 2010, Astrophys. J. 722, 954.

Roca-Maza, X., M. Brenna, B. K. Agrawal, P. F. Bortignon, G. Colò, L.-G. Cao, N. Paar, and D. Vretenar, 2013, Phys. Rev. C 87, 034301.

Roca-Maza, X., M. Centelles, X. Viñas, M. Brenna, G. Colò, B. K. Agrawal, N. Paar, J. Piekarewicz, and D. Vretenar, 2013, Phys. Rev. C 88, 024316.

Roca-Maza, X., M. Centelles, X. Viñas, and M. Warda, 2011, Phys. Rev. Lett. 106, 252501.

Roca-Maza, X., and J. Piekarewicz, 2008, Phys. Rev. C 78, 025807. Roca-Maza, X., J. Piekarewicz, T. Garcia-Galvez, and M. Centelles, 2012, in Neutron star crust, edited by J.P.C. Bertulani (Nova Science Publishers, New York).

Roca-Maza, X., X. Viñas, M. Centelles, B. K. Agrawal, G. Colo', N. Paar, J. Piekarewicz, and D. Vretenar, 2015, Phys. Rev. C 92, 064304.
Roca-Maza, X., X. Viñas, M. Centelles, P. Ring, and P. Schuck, 2011, Phys. Rev. C 84, 054309.

Roggero, A., A. Mukherjee, and F. Pederiva, 2014, Phys. Rev. Lett. 112, 221103.

Romani, R. W., A. V. Filippenko, J. M. Silverman, S. B. Cenko, J. Greiner, A. Rau, J. Elliott, and H. J. Pletsch, 2012, Astrophys. J. Lett. 760, L36.

Röpke, G., 2009, Phys. Rev. C 79, 014002.

Röpke, G., 2011, Nucl. Phys. A 867, 66.

Röpke, G., L. Münchow, and H. Schulz, 1982, Nucl. Phys. A 379, 536.

Röpke, G., M. Schmidt, L. Münchow, and H. Schulz, 1983, Nucl. Phys. A 399, 587.

Rosswog, S., 2015, Int. J. Mod. Phys. D 24, 1530012.

Rosswog, S., T. Piran, and E. Nakar, 2013, Mon. Not. R. Astron. Soc. 430, 2585.

Rotondo, M., J. A. Rueda, R. Ruffini, and S.-S. Xue, 2011, Phys. Rev. C 83, 045805.

Ruester, S. B., M. Hempel, and J. Schaffner-Bielich, 2006, Phys. Rev. C 73, 035804

Rufa, M., P. Reinhard, J. Maruhn, W. Greiner, and M. Strayer, 1988, Phys. Rev. C 38, 390.

Rusnak, J. J., and R. Furnstahl, 1997, Nucl. Phys. A 627, 495.

Russotto, P., et al., 2011, Phys. Lett. B 697, 471.

Russotto, P., et al., 2014, Eur. Phys. J. A 50, 38.

Russotto, P., et al. (ASY-EOS Collaboration), 2013, J. Phys. Conf. Ser. 420, 012092.

Sagert, I., T. Fischer, M. Hempel, G. Pagliara, J. Schaffner-Bielich, A. Mezzacappa, F.-K. Thielemann, and M. Liebendörfer, 2009, Phys. Rev. Lett. 102, 081101.

Sagert, I., T. Fischer, M. Hempel, G. Pagliara, J. Schaffner-Bielich, F.-K. Thielemann, and M. Liebendörfer, 2010, J. Phys. G 37, 094064.

Sagert, I., L. Tolos, D. Chatterjee, J. Schaffner-Bielich, and C. Sturm, 2012, Phys. Rev. C 86, 045802.

Sagert, I., et al., 2012, Acta Phys. Pol. B 43, 741.

Sagun, V. V., A. I. Ivanytskyi, K. A. Bugaev, and I. N. Mishustin, 2014, Nucl. Phys. A 924, 24.

Saha, P. K., et al., 2004, Phys. Rev. C 70, 044613.

Salpeter, E., 1961, Astrophys. J. 134, 669.

Sammarruca, F., 2010, Int. J. Mod. Phys. E 19, 1259.

Sammarruca, F., B. Chen, L. Coraggio, N. Itaco, and R. Machleidt, 2012, Phys. Rev. C 86, 054317.

Schaefer, B.-J., M. Wagner, J. Wambach, T. Kuo, and G. Brown, 2006, Phys. Rev. C 73, 011001.

Schaffner, J., and I. N. Mishustin, 1996, Phys. Rev. C 53, 1416.

Schaffner, J., et al., 1994, Ann. Phys. (N.Y.) 235, 35.

Schaffner-Bielich, J., and A. Gal, 2000, Phys. Rev. C 62, 034311.

Schaffner-Bielich, J., M. Hanauske, H. Stoecker, and W. Greiner, 2002, Phys. Rev. Lett. 89, 171101.

Scheidegger, S., R. Käppeli, S. C. Whitehouse, T. Fischer, and M. Liebendörfer, 2010, Astron. Astrophys. 514, A51.

Schmidt, K. E., and S. Fantoni, 1999, Phys. Lett. B 446, 99.

Schmidt, M., G. Röpke, and H. Schulz, 1990, Ann. Phys. (N.Y.) 202, 57.

Schmidt, S., D. Blaschke, and Y. Kalinovsky, 1994, Phys. Rev. C 50, 435.

Schneider, A., D. Berry, C. Briggs, M. Caplan, and C. Horowitz, 2014, Phys. Rev. C 90, 055805.

Schneider, A., C. Horowitz, J. Hughto, and D. Berry, 2013, Phys. Rev. C 88, 065807.

Schuetrumpf, B., et al., 2013a, J. Phys. Conf. Ser. 426, 012009.

Schuetrumpf, B., et al., 2013b, Phys. Rev. C 87, 055805. 
Schuetrumpf, B., et al., 2015, Phys. Rev. C 91, 025801.

Schulze, H.-J., and T. Rijken, 2011, Phys. Rev. C 84, 035801.

Schwenk, A., 2005, J. Phys. G 31, S1273.

Sebille, F., V. de la Mota, and S. Figerou, 2011, Phys. Rev. C 84, 055801.

Sebille, F., S. Figerou, and V. de la Mota, 2009, Nucl. Phys. A 822, 51 .

Sechi-Zorn, B., B. Kehoe, J. Twitty, and R. Burnstein, 1968, Phys. Rev. 175, 1735.

Sedrakian, A., 2007, Prog. Part. Nucl. Phys. 58, 168.

Sedrakian, A., 2013, Astron. Astrophys. 555, L10.

Seitenzahl, I. R., D. M. Townsley, F. Peng, and J. W. Truran, 2009, At. Data Nucl. Data Tables 95, 96.

Sekiguchi, Y., K. Kiuchi, K. Kyutoku, and M. Shibata, 2011, Phys. Rev. Lett. 107, 211101.

Sekiguchi, Y., K. Kiuchi, K. Kyutoku, and M. Shibata, 2015, Phys. Rev. D 91, 064059.

Sekiguchi, Y., and M. Shibata, 2011, Astrophys. J. 737, 6.

Sellahewa, R., and A. Rios, 2014, Phys. Rev. C 90, 054327.

Serot, B. D., 1992, Rep. Prog. Phys. 55, 1855.

Serot, B. D., and J. D. Walecka, 1986, Adv. Nucl. Phys. 16, 1.

Serot, B. D., and J. D. Walecka, 1997, Int. J. Mod. Phys. E 06, 515.

Sharma, B. K., M. Centelles, X. Viñas, M. Baldo, and G. F. Burgio, 2015, Astron. Astrophys. 584, A103.

Sharma, M. M., 2009, Nucl. Phys. A 816, 65.

Shen, G., and C. Horowitz, 2010, “Gang Shen EOS Web Site," http:// cecelia.physics.indiana.edu/gang_shen_eos.

Shen, G., C. Horowitz, and S. Teige, 2010, Phys. Rev. C 82, 015806.

Shen, G., C. J. Horowitz, and E. O'Connor, 2011, Phys. Rev. C 83, 065808.

Shen, G., C. J. Horowitz, and S. Teige, 2011, Phys. Rev. C 83, 035802.

Shen, H., H. Toki, K. Oyamatsu, and K. Sumiyoshi, 1998a, Nucl. Phys. A 637, 435.

Shen, H., H. Toki, K. Oyamatsu, and K. Sumiyoshi, 1998b, Prog. Theor. Phys. 100, 1013.

Shen, H., H. Toki, K. Oyamatsu, and K. Sumiyoshi, 2011, Astrophys. J. Suppl. Ser. 197, 20.

Shetty, D. V., S. J. Yennello, and G. A. Souliotis, 2007, Phys. Rev. C 76, 024606 [76, 039902(E) (2007)].

Shibata, M., and K. Taniguchi, 2011, Living Rev. Relativ. 14, 6.

Shlomo, S., V. M. Kolomietz, and G. Colò, 2006, Eur. Phys. J. A 30, 23.

Shternin, P. S., D. G. Yakovlev, C. O. Heinke, W. C. Ho, and D. J. Patnaude, 2011, Mon. Not. R. Astron. Soc. 412, L108.

Sil, T., et al., 2002, Phys. Rev. C 66, 045803.

Skyrme, T., 1956, Philos. Mag. 1, 1043.

Skyrme, T., 1959, Nucl. Phys. 9, 615.

Sonoda, H., G. Watanabe, K. Sato, K. Yasuoka, and T. Ebisuzaki, 2008, Phys. Rev. C 77, 035806.

Sotani, H., K. Iida, K. Oyamatsu, and A. Ohnishi, 2014, Prog. Theor. Exp. Phys. 2014, 051E01.

Sotani, H., K. Nakazato, K. Iida, and K. Oyamatsu, 2012, Phys. Rev. Lett. 108, 201101.

Sotani, H., K. Nakazato, K. Iida, and K. Oyamatsu, 2013a, Mon. Not. R. Astron. Soc. Lett. 428, L21.

Sotani, H., K. Nakazato, K. Iida, and K. Oyamatsu, 2013b, Mon. Not. R. Astron. Soc. 434, 2060.

Souza, S. R., A. W. Steiner, W. G. Lynch, R. Donangelo, and M. A. Famiano, 2009, Astrophys. J. 707, 1495.

Stein, H., K. Morawetz, and G. Röpke, 1997, Phys. Rev. A 55, 1945.

Stein, H., A. Schnell, T. Alm, and G. Röpke, 1995, Z. Phys. A 351, 295.
Steiner, A., M. Prakash, and J. M. Lattimer, 2000, Phys. Lett. B 486, 239.

Steiner, A. W., 2008, Phys. Rev. C 77, 035805.

Steiner, A. W., and S. Gandolfi, 2012, Phys. Rev. Lett. 108, 081102.

Steiner, A. W., M. Hempel, and T. Fischer, 2013, Astrophys. J. 774, 17.

Steiner, A. W., J. M. Lattimer, and E. F. Brown, 2010, Astrophys. J. 722, 33.

Steiner, A. W., J. M. Lattimer, and E. F. Brown, 2013, Astrophys. J. 765, L5.

Steiner, A. W., M. Prakash, J. M. Lattimer, and P. J. Ellis, 2005, Phys. Rep. 411, 325.

Steiner, A. W., and A. L. Watts, 2009, Phys. Rev. Lett. 103, 181101.

Stergioulas, N., 1996, "RNS: Rapidly Rotating Neutron Stars," http:// www.gravity.phys.uwm.edu/rns.

Stoitsov, M., et al., 2010, Phys. Rev. C 82, 054307.

Stoks, V., R. Kompl, M. Rentmeester, and J. de Swart, 1993, Phys. Rev. C 48, 792.

Stone, J., 2005, J. Phys. G 31, R211.

Stone, J., and P.-G. Reinhard, 2007, Prog. Part. Nucl. Phys. 58, 587.

Stone, J., N. Stone, and S. Moszkowski, 2014, Phys. Rev. C 89, 044316.

Strachan, A., and C. Dorso, 1997, Phys. Rev. C 56, 995.

Sturm, C. T., et al. (KAOS Collaboration), 2001, Phys. Rev. Lett. 86, 39.

Sugahara, Y., and H. Toki, 1994, Nucl. Phys. A 579, 557.

Sugimura, H., et al. (J-PARC E10 Collaboration), 2014, Phys. Lett. B 729, 39.

Sullivan, C., E. O'Connor, R. G. T. Zegers, T. Grubb, and S. M. Austin, 2016, Astrophys. J. 816, 44.

Sumiyoshi, K., 1998, Home page of Relativistic EoS, http://user .numazu-ct.ac.jp/ sumi/eos/index.html.

Sumiyoshi, K., C. Ishizuka, A. Ohnishi, S. Yamada, and H. Suzuki, 2009, Astrophys. J. Lett. 690, L43.

Sumiyoshi, K., K. Oyamatsu, and H. Toki, 1995, Nucl. Phys. A 595, 327.

Sumiyoshi, K., and G. Röpke, 2008, Phys. Rev. C 77, 055804.

Sumiyoshi, K., S. Yamada, and H. Suzuki, 2007, Astrophys. J. 667, 382.

Sumiyoshi, K., S. Yamada, H. Suzuki, H. Shen, S. Chiba, and H. Toki, 2005, Astrophys. J. 629, 922.

Sun, Z. Y., et al., 2010, Phys. Rev. C 82, 051603.

Suwa, Y., 2014, Publ. Astron. Soc. Jpn. 66, L1.

Suwa, Y., et al., 2013, Astrophys. J. 764, 99.

Takami, K., L. Rezzolla, and L. Baiotti, 2014, Phys. Rev. Lett. 113, 091104.

Tamii, A., P. von Neumann-Cosel, and I. Poltoratska, 2014, Eur. Phys. J. A 50, 28.

Tamii, A., et al., 2011, Phys. Rev. Lett. 107, 062502.

Tanvir, N. R., A. J. Levan, A. S. Fruchter, J. Hjorth, R. A. Hounsell, K. Wiersema, and R. L. Tunnicliffe, 2013, Nature (London) 500, 547.

Tarbert, C., et al., 2014, Phys. Rev. Lett. 112, 242502.

Tauris, T. M., N. Langer, T. J. Moriya, P. Podsiadlowski, S.-C. Yoon, and S. I. Blinnikov, 2013, Astrophys. J. Lett. 778, L23.

Terashima, S., et al., 2008, Phys. Rev. C 77, 024317.

Ter Haar, B., and R. Malfliet, 1987, Phys. Rep. 149, 207.

Tews, I., T. Krüger, K. Hebeler, and A. Schwenk, 2013, Phys. Rev. Lett. 110, 032504.

Thomas, A., D. Whittenbury, J. Carroll, K. Tsushima, and J. Stone, 2013, EPJ Web Conf. 63, 03004.

Thouless, D. J., 1960, Ann. Phys. (N.Y.) 10, 553. 
Todd-Rutel, B., and J. Piekarewicz, 2005, Phys. Rev. Lett. 95, 122501.

Togashi, H., and M. Takano, 2013, Nucl. Phys. A 902, 53.

Togashi, H., M. Takano, K. Sumiyoshi, and K. Nakazato, 2014, Prog. Theor. Exp. Phys. 2014, 023 D05.

Toki, H., D. Hirata, Y. Sugahara, K. Sumiyoshi, and I. Tanihata, 1995, Nucl. Phys. A 588, c357.

Tolman, R. C., 1939, Phys. Rev. 55, 364.

Tolos, L., B. Friman, and A. Schwenk, 2008, Nucl. Phys. A 806, 105.

Trippa, L., G. Colò, and E. Vigezzi, 2008, Phys. Rev. C 77, 061304.

Trzcinska, A., et al., 2001, Phys. Rev. Lett. 87, 082501.

Tsang, M., et al., 2009, Phys. Rev. Lett. 102, 122701.

Tsang, M., et al., 2012, Phys. Rev. C 86, 015803.

Tsang, M. B., et al., 2004, Phys. Rev. Lett. 92, 062701.

Typel, S., 2005, Phys. Rev. C 71, 064301.

Typel, S., 2013, AIP Conf. Proc. 1520, 68.

Typel, S., 2014, Phys. Rev. C 89, 064321.

Typel, S., 2016, Eur. Phys. J. A 52, 16.

Typel, S., and B. A. Brown, 2001, Phys. Rev. C 64, 027302.

Typel, S., M. Oertel, and T. Klähn, 2015, Phys. Part. Nucl. 46, 633.

Typel, S., G. Röpke, T. Klähn, D. Blaschke, and H. Wolter, 2010, Phys. Rev. C 81, 015803.

Typel, S., T. von Chossy, and H. Wolter, 2003, Phys. Rev. C 67, 034002.

Typel, S., and H. Wolter, 1999, Nucl. Phys. A 656, 331.

Typel, S., H. Wolter, G. Röpke, and D. Blaschke, 2014, Eur. Phys. J. A 50, 17.

van Dalen, E., G. Colucci, and A. Sedrakian, 2014, Phys. Lett. B 734, 383.

van Dalen, E., C. Fuchs, and A. Faessler, 2004, Nucl. Phys. A 744, 227.

van Kerkwijk, M., R. Breton, and S. Kulkarni, 2011, Astrophys. J. 728, 95

Vantournhout, K., and H. Feldmeier, 2012, J. Phys. Conf. Ser. 342, 012011.

Vantournhout, K., N. Jachowicz, and J. Ryckebusch, 2011, Phys. Rev. C 84, 032801.

Vantournhout, K., T. Neff, H. Feldmeier, N. Jachowicz, and J. Ryckebusch, 2011, Prog. Part. Nucl. Phys. 66, 271.

Vautherin, D., and D. Brink, 1970, Phys. Lett. B 32, 149.

Vautherin, D., and D. Brink, 1972, Phys. Rev. C 5, 626.

Venugopalan, R., and M. Prakash, 1992, Nucl. Phys. A 546, 718.

Verbiest, J., et al., 2008, Astrophys. J. 679, 675.

Vidaña, I., 2012, Phys. Rev. C 85, 045808 [90, 029901(E) (2014)].

Vidaña, I., D. Logoteta, C. Providençia, A. Polls, and I. Bombaci, 2010, arXiv:1004.3958

Vidaña, I., D. Logoteta, C. Providençia, A. Polls, and I. Bombaci, 2011, Europhys. Lett. 94, 11002.

Vidaña, I., A. Polls, A. Ramos, and H. J. Schulze, 2001, Phys. Rev. C 64, 044301

Viñas, X., M. Centelles, X. Roca-Maza, and M. Warda, 2014a, AIP Conf. Proc. 1606, 256.

Viñas, X., M. Centelles, X. Roca-Maza, and M. Warda, 2014b, Eur. Phys. J. A 50, 27.

Voskresenskaya, M., and S. Typel, 2012, Nucl. Phys. A 887, 42.

Voskresensky, D. N., M. Yasuhira, and T. Tatsumi, 2003, Nucl. Phys. A 723, 291.

Vretenar, D., A. Afanasjev, G. Lalazissis, and P. Ring, 2005, Phys. Rep. 409, 101.

Wada, R., et al., 2012, Phys. Rev. C 85, 064618.

Wagner, M., B.-J. Schaefer, J. Wambach, T. Kuo, and G. Brown, 2006, Phys. Rev. C 74, 054003.

Walecka, J., 1974, Ann. Phys. (N.Y.) 83, 491
Walhout, T., R. Cenni, A. Fabrocini, and S. Fantoni, 1996, Phys. Rev. C 54, 1622.

Wanajo, S., et al., 2014, Astrophys. J. 789, L39.

Wang, J.-c., Q. Wang, and D. H. Rischke, 2011, Phys. Lett. B 704, 347.

Wang, M., G. Audi, A. H. Wapstra, F. G. Kondev, M. MacCormick, X. Xu, and B. Pfeiffer, 2012, Chin. Phys. C 36, 1603.

Wang, N., and T. Li, 2013, Phys. Rev. C 88, 011301.

Wang, N., L. Ou, and M. Liu, 2013, Phys. Rev. C 87, 034327.

Warda, M., X. Viñas, X. Roca-Maza, and M. Centelles, 2009, Phys. Rev. C 80, 024316.

Warda, M., X. Viñas, X. Roca-Maza, and M. Centelles, 2010, Phys. Rev. C 81, 054309.

Washiyama, K., et al., 2012, Phys. Rev. C 86, 054309.

Watanabe, G., 2007, AIP Conf. Proc. 891, 373.

Watanabe, G., and K. Iida, 2003, Phys. Rev. C 68, 045801.

Watanabe, G., K. Iida, and K. Sato, 2000, Nucl. Phys. A 676, 455.

Watanabe, G., T. Maruyama, K. Sato, K. Yasuoka, and T. Ebisuzaki, 2005, Phys. Rev. Lett. 94, 031101.

Watanabe, G., K. Sato, K. Yasuoka, and T. Ebisuzaki, 2002a, Prog. Theor. Phys. Suppl. 146, 638.

Watanabe, G., K. Sato, K. Yasuoka, and T. Ebisuzaki, 2002b, Phys. Rev. C 66, 012801.

Watanabe, G., K. Sato, K. Yasuoka, and T. Ebisuzaki, 2003a, Nucl. Phys. A 718, 700.

Watanabe, G., K. Sato, K. Yasuoka, and T. Ebisuzaki, 2003b, Phys. Rev. C 68, 035806.

Watanabe, G., K. Sato, K. Yasuoka, and T. Ebisuzaki, 2004, Phys. Rev. C 69, 055805.

Watanabe, G., et al., 2009, Phys. Rev. Lett. 103, 121101.

Weber, F., 1999, Pulsars as astrophysical laboratories for nuclear and particle physics (Institute of Physics Publishing, Bristol, UK).

Weber, F., 2005, Prog. Part. Nucl. Phys. 54, 193.

Weinberg, S., 1990, Phys. Lett. B 251, 288.

Weinberg, S., 1991, Nucl. Phys. B 363, 3.

Weissenborn, S., D. Chatterjee, and J. Schaffner-Bielich, 2012a, Nucl. Phys. A 881, 62.

Weissenborn, S., D. Chatterjee, and J. Schaffner-Bielich, 2012b, Phys. Rev. C 85, 065802.

Weissenborn, S., I. Sagert, G. Pagliara, M. Hempel, and J. SchaffnerBielich, 2011, Astrophys. J. 740, L14.

Welke, G. M., M. Prakash, T. T. S. Kuo, S. Das Gupta, and C. Gale, 1988, Phys. Rev. C 38, 2101.

Wellenhofer, C., J. W. Holt, and N. Kaiser, 2015, Phys. Rev. C 92 , 015801 .

Wellenhofer, C., J. W. Holt, N. Kaiser, and W. Weise, 2014, Phys. Rev. C 89, 064009.

Wen, D.-H., W. G. Newton, and B.-A. Li, 2012, Phys. Rev. C 85, 025801.

Whittenbury, D., J. Carroll, A. Thomas, K. Tsushima, and J. Stone, 2014, Phys. Rev. C 89, 065801.

Wienholtz, F., et al., 2013, Nature (London) 498, 346.

Williams, R., and S. Koonin, 1985, Nucl. Phys. A 435, 844.

Wiringa, R., R. Schiavilla, S. C. Pieper, and J. Carlson, 2014, Phys. Rev. C 89, 024305.

Wiringa, R. B., and S. C. Pieper, 2002, Phys. Rev. Lett. 89, 182501.

Wiringa, R. B., R. Smith, and T. Ainsworth, 1984, Phys. Rev. C 29, 1207.

Wiringa, R. B., V. Stoks, and R. Schiavilla, 1995, Phys. Rev. C 51, 38.

Witten, E., 1984, Phys. Rev. D 30, 272.

Wlazłowski, G., and P. Magierski, 2011, Phys. Rev. C 83, 012801. 
Wlazłowski, G., J. Holt, S. Moroz, A. Bulgac, and K. Roche, 2014, Phys. Rev. Lett. 113, 182503.

Wolf, R., et al. (ISOLTRAP Collaboration), 2013, Phys. Rev. Lett. 110, 041101.

Wolff, R. G., 1980, Diplomathesis, T. H. Darmstadt (unpublished).

Wolter, H., et al., 2009, Prog. Part. Nucl. Phys. 62, 402.

Woosley, S. E., and T. A. Weaver, 1995, Astrophys. J. Suppl. Ser. 101, 181.

Xiao, Z., B.-A. Li, L.-W. Chen, G.-C. Yong, and M. Zhang, 2009, Phys. Rev. Lett. 102, 062502.

Xiao, Z.-G., et al., 2014, Eur. Phys. J. A 50, 37.

Xie, W.-J., J. Su, L. Zhu, and F.-S. Zhang, 2013, Phys. Lett. B 718, 1510. Xu, C., B.-A. Li, and L.-W. Chen, 2010, Phys. Rev. C 82, 054607.

Xu, J., L.-W. Chen, and B.-A. Li, 2015, Phys. Rev. C 91, 014611.

Yakovlev, D. G., and C. Pethick, 2004, Annu. Rev. Astron. Astrophys. 42, 169.

Yamamoto, Y., S.-i. Fujimoto, H. Nagakura, and S. Yamada, 2013, Astrophys. J. 771, 27.

Yang, B., Z.-P. Jin, X. Li, S. Covino, X.-Z. Zheng, K. Hotokezaka, Y.-Z. Fan, T. Piran, and D.-M. Wei, 2015, Nat. Commun. 6, 7323.

Yasutake, N., and K. Kashiwa, 2009, Phys. Rev. D 79, 043012.

Yasutake, N., T. Maruyama, and T. Tatsumi, 2011, J. Phys. Conf. Ser. 312, 042027.

Yasutake, N., T. Noda, H. Sotani, T. Maruyama, and T. Tatsumi, 2013, in Recent Advances in Quarks Research (Nova Science Publishers, New York), pp. 63-111.
Yong, G.-C., B.-A. Li, L.-W. Chen, and X.-C. Zhang, 2009, Phys. Rev. C 80, 044608.

Yudin, A., 2010, "Excluded volume approximation for supernova matter," in $\operatorname{PoS}(\mathrm{NIC} X I) 15$.

Yudin, A. V., 2011, Astron. Lett. 37, 576.

Yukawa, H., 1955, Prog. Theor. Phys. 1, 1.

Zacchi, A., R. Stiele, and J. Schaffner-Bielich, 2015, Phys. Rev. D 92, 045022 .

Zdunik, J., 2000, Astron. Astrophys. 359, 311.

Zdunik, J., and P. Haensel, 2013, Astron. Astrophys. 551, A61.

Zenihiro, J., et al., 2010, Phys. Rev. C 82, 044611.

Zhang, Y., M. Tsang, Z. Li, and H. Liu, 2014, Phys. Lett. B 732 , 186.

Zhang, Z., and L.-W. Chen, 2013, Phys. Lett. B 726, 234.

Zhang, Z., and L.-W. Chen, 2015, Phys. Rev. C 92, 031301.

Zhang, Z., and H. Shen, 2014, Astrophys. J. 788, 185.

Zhao, P., Z. Li, J. Yao, and J. Meng, 2010, Phys. Rev. C 82, 054319.

Zhou, X. R., G. F. Burgio, U. Lombardo, H.-J. Schulze, and W. Zuo, 2004, Phys. Rev. C 69, 018801.

Zimanyi, J., and S. Moszkowski, 1990, Phys. Rev. C 42, 1416.

Zuo, W., A. Lejeune, U. Lombardo, and J. Mathiot, 2002a, Nucl. Phys. A 706, 418.

Zuo, W., A. Lejeune, U. Lombardo, and J. Mathiot, 2002b, Eur. Phys. J. A 14, 469. 